O nordon

Per- and polyfluorinated substances in the Nordic Countries

Use, occurence and toxicology 

2 norden 



\title{
Per- and polyfluorinated substances in the Nordic Countries
}

\author{
Use, occurence and toxicology
}

Stefan Posner and Sandra Roos at Swerea IVF. Pia Brunn Poulsen at FORCE Technology. Hrönn Ólína Jörundsdottir and Helga Gunnlaugsdóttir at Matís ohf/Icelandic Food and Biotech R\&D. Xenia Trier at the Technical University of Denmark (DTU). Allan Astrup Jensen at Nordic Institute of Product Sustainability, Environmental Chemistry and Toxicology (NIPSECT). Athanasios A. Katsogiannis and Dorte Herzke at NILU (Norwegian Institute for Air Reasearch). Eva Cecilie Bonefeld-Jörgensen at the University of Aarhus. Christina Jönsson at Swerea IVF. Gitte Alsing Pedersen, DTU. Mandana Ghisari, University of Århus. Sophie Jensen, Matis

TemaNord 2013:542 
Per- and polyfluorinated substances in the Nordic Countries

Use, occurence and toxicology

Stefan Posner and Sandra Roos at Swerea IVF. Pia Brunn Poulsen at FORCE Technology. Hrönn Ólína Jörundsdottir and Helga Gunnlaugsdóttir at Matís ohf/Icelandic Food and Biotech R\&D. Xenia Trier at the Technical University of Denmark (DTU). Allan Astrup Jensen at Nordic Institute of Product Sustainability, Environmental Chemistry and Toxicology (NIPSECT). Athanasios A. Katsogiannis and Dorte Herzke at NILU (Norwegian Institute for Air Reasearch). Eva Cecilie Bonefeld-Jörgensen at the University of Aarhus. Christina Jönsson at Swerea IVF. Gitte Alsing Pedersen, DTU. Mandana Ghisari, University of Århus. Sophie Jensen, Matis

ISBN 978-92-893-2562-2

http://dx.doi.org/10.6027/TN2013-542

TemaNord 2013:542

(C) Nordic Council of Ministers 2013

Layout: Hanne Lebech

Cover photo: KLIF

This publication has been published with financial support by the Nordic Council of Ministers. However, the contents of this publication do not necessarily reflect the views, policies or recommendations of the Nordic Council of Ministers.

\section{www.norden.org/en/publications}

\section{Nordic co-operation}

Nordic co-operation is one of the world's most extensive forms of regional collaboration, involving Denmark, Finland, Iceland, Norway, Sweden, and the Faroe Islands, Greenland, and Åland.

Nordic co-operation has firm traditions in politics, the economy, and culture. It plays an important role in European and international collaboration, and aims at creating a strong Nordic community in a strong Europe.

Nordic co-operation seeks to safeguard Nordic and regional interests and principles in the global community. Common Nordic values help the region solidify its position as one of the world's most innovative and competitive.

\section{Nordic Council of Ministers}

Ved Stranden 18

DK-1061 Copenhagen K

Phone (+45) 33960200

\section{www.norden.org}




\section{Content}

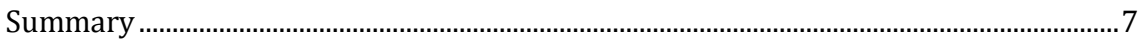

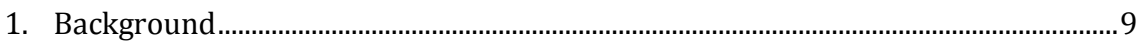

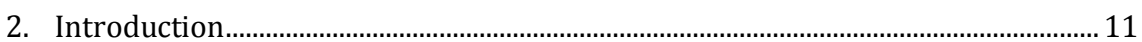

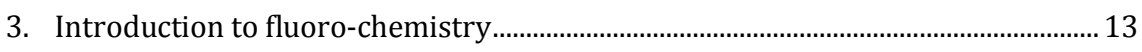

3.1 Production of fluoro-chemicals ....................................................................... 14

4. Methodology and limitations........................................................................................ 19

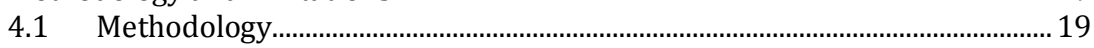

4.2 Limitations....................................................................................................... 20

5. Mapping of use of per- and polyfluorinated substances on the Nordic

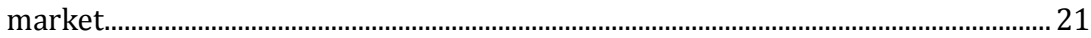

5.1 "Net list" of PFCs in use on the Nordic market.............................................. 21

5.2 Contacts to producers, suppliers, users and other players on the

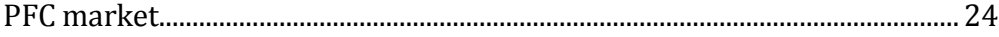

5.3 Conclusions.................................................................................................. 25

6. Mapping of uses and applications of PFCs on the Nordic market ............................. 27

6.1 Aviation hydraulic fluids.................................................................................. 27

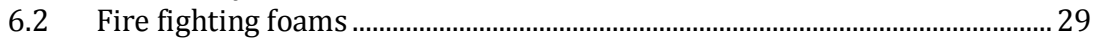

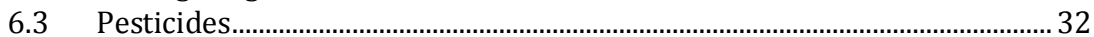

6.4 Metal plating (hard metal plating and decorative plating)........................ 33

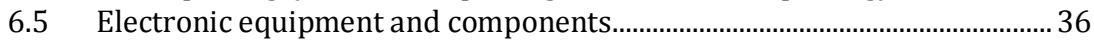

6.6 Chemically driven oil and mining production................................................... 37

6.7 Carpets, leather and apparel, textiles and upholstery.....................................3 38

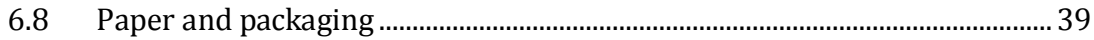

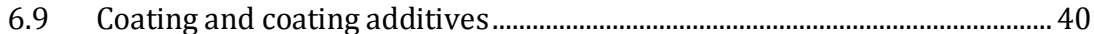

6.10 Others ............................................................................................................. 42

6.11 Other important market information for the Nordic market ......................... 43

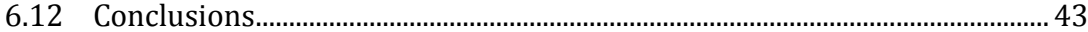

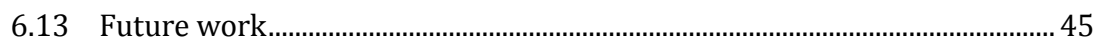

7. Occurrence of per- and polyfluorinated substances ................................................... 47

7.1 Emissions to and occurrence of PFCs into the environment ........................4 47

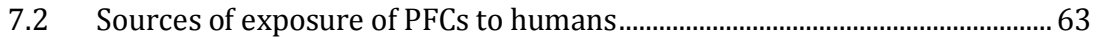

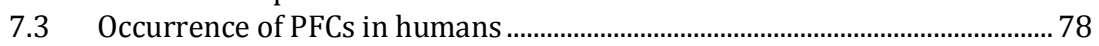

7.4 Suggested priority list of substances..............................................................103

7.5 Overall conclusion for the human biomonitoring data on PFCA,

PFSA and other PFC telomers ..........................................................................103

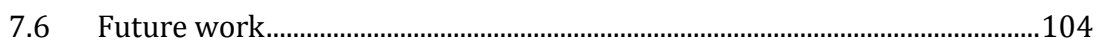


8. Human health effects and related animal toxicity of per- and

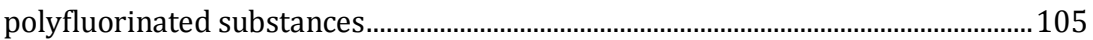

8.1 PFCA (Perfluoroalkyl carboxylates) …….....................................................105

8.2 PFSA (Perfluoroalkyl sulfonates) ……….........................................................123

8.3 FTOH (fluorotelomer alcohols) ...................................................................... 131

8.4 FTS (fluorotelomer sulfonates) .......................................................................133

8.5 PAP/di-PAP (polyfluoroalkyl phosphate esters) ............................................133

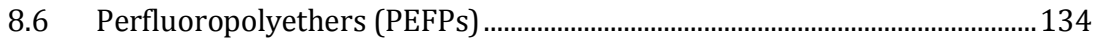

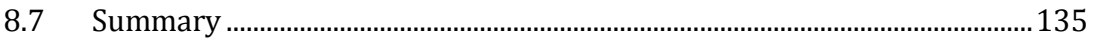

9. Environmental effects of per- and polyfluorinated substances.............................147

9.1 Perfluoro carboxylates (PFCAs) …………......................................................148

9.2 Perfluoroalkyl sulfonates (PFSAs) ...............................................................149

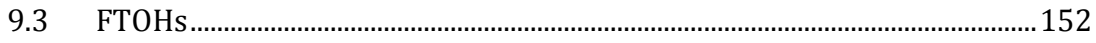

9.4 Other fluorinated compounds of interest....................................................153

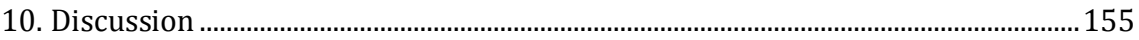

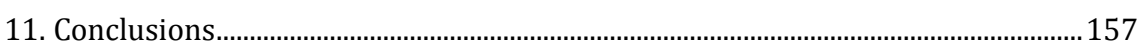

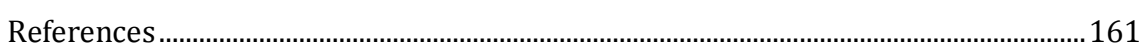

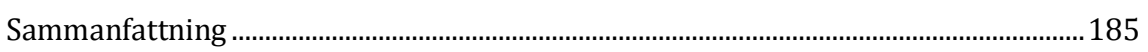

Appendix A - List of abbreviations and acronyms......................................................... 187

Appendix B - Illustration of mapping of SPIN- and preregistered chemicals.............191

Appendix C - List of contacted companies/institutions .................................................20

Appendix D - Commercial PFC products and brands on the market.............................209

Appendix E - Data contributions to "Mapping of uses and applications of PFCs on the Nordic market" ...............................................................................................213

Appendix F - Data contributions of PFCA and PFSA in food and drinking water. 


\section{Summary}

The Nordic Chemicals Group (NKG), which is subordinate to the Nordic Council of Ministers, has commissioned the authors, through the Climate and Pollution Agency (KLIF), to undertake a Nordic study based on open information sources and custom market research to describe the use and occurrence of the most common perfluorinated substances (PFC), with less focus on PFOS and PFOA.

The study includes three stages:

1. Identification of relevant per-and polyfluorinated substances and their use in various industrial sectors in the Nordic market.

2. Occurrence in industrial and consumer products and potential emissions to and in the Nordic environment and humans of the substances described in stage 1 .

3. A summary of knowledge of the toxic effects on humans and the environment of substances prioritized in stage 2 .

Interviews were conducted with more than 50 players in the Nordic market with the aim of obtaining information on use and type of PFC substances. This study, however, gave poor results. In parallel with this survey a net list was therefore produced of PFC substances based on three lists (each separately and together incomplete) from the OECD, REACH pre-registration database, and the Nordic SPIN database. Most production of PFC containing articles is outside the EU and today's legal framework does not provide adequate means to obtain sufficient information about specific PFC substances in imported articles. This net list is therefore not complete so there may be significantly more PFC substances used in the Nordic market.

There are relatively few studies on PFC substances in the environment in the Nordic countries other than PFOA and PFOS which include both biotic (air, land and water) and abiotic (animal and human) data.

Most human data regarding PFCA and PFSA from the years 1992 to 2010 are from Norway and Sweden, with fewer from Denmark and no data from Iceland and Finland. Regarding PFCAs, most studies show the occurrence of PFOA, PFNA and PFHXA. However other PFCA substances (C10-C13) have also been detected in a number of studies. Regarding 
PFSA, PFOS and PFHxS are the most studied substances. Human data are missing for PFAL, FTS, PAP/di-PAP and FTMAPs.

In comparison with long-chain PFC substances ( $\geq$ C8) the short-chain substances are considered to be less toxic but a number of studies indicate both ecotoxicity and human toxicity. In this area there is a major lack of studies.

In general, since 2002 decreasing levels of PFOA and PFOS are observed in the environment. However, increasing levels of short chained sulfonates have been observed in the environment. In comparison with other countries, the background concentrations of PFOA and PFOS in the environment are lower in the Scandinavian countries especially compared with Central European countries, which is to be expected as populations are smaller and there is less industry in the Nordic countries. However these substances have also been found in the Arctic, far from any sources, which shows that these substances are global contaminants.

One result of this review of the presence of fluorinated substances in the environment is that there are considerable information and knowledge gaps regarding PFCs other than PFOA and PFOS. In addition, there is generally a shortage of human and environmental data about these PFCs. The few data available indicate specific toxic effects on humans and the environment. It takes more and deeper studies to get a clearer picture of these PFC substances before far-reaching conclusions can be drawn about their toxic properties.

Lack of physical-chemical data for PFC substances other than PFOA and PFOS is an obstacle to environmental fate modelling calculations.

The lack of analytical reference substances is currently also a barrier to extended studies of these substances in the environment and humans. 


\section{Background}

Polyfluorinated substances have been used for a long time, but there was no focus on this group until widespread environmental occurrence (e.g., in polar bears) and high reproductive toxicity were found for perfluorooctane sulfonate (PFOS). Because of these properties of the extremely persistent PFOS and by the fact that PFCs do not occur naturally in nature, the substance is restricted under the Stockholm Convention (nominated by Sweden), with only a few allowed remaining uses. Perfluorooctanoic acid (PFOA) was the second substance from this group to attract interest, with hazard and risk assessments being performed, and classification and labelling under discussion in the EU (proposal from Norway). PFOA is a candidate for restriction under Reach. The OECD (Organization for Economic Cooperation and Development) lists a total of 853 different fluorine compounds. Among these some are currently being phased out due to regulations mentioned above.

However, there is a huge number of polyfluorinated substances (including perfluorinated) being used, in many cases leading to substitution of one polyfluorinated substance with others, e.g., perfluorobutansulfonate (PFBS) substituting PFOS. Little is known about the sources of these substances. Many other perfluorinated substances are known to be used, but it is unclear to what extent they are included in monitoring/screening exercises.

Some widely used polyfluorinated substances such as fluorotelomer alcohol-derivatives are precursors to perfluorinated substances. Examples from these groups are polyfluorinated phosphates (diPAPs and PAPs), and fluorotelomer mercaptoalkyl phosphate diesters (FTMAPs), found in food contact materials by Danish scientists (Trier 2011). The polyfluorinated substances are rather persistent but may be degraded to perfluorinated substances, such as PFOA, which in itself is virtually nondegradable and may be problematic as such. In addition, sufficient toxicity data is only available for very few of them.

The overall publicly available knowledge on the use of per- and polyfluorinated substances is very limited, even though we know that there are many such substances on the market. This review aims to increase our knowledge of the uses of these fluorinated substitutes of PFOS/PFOA. This includes emissions and exposures in the Nordic envi- 
ronment, and if available, more information on the toxicity and monitoring results of these substances. Of special concern is whether some of the perfluorinated substances already have contaminated the Arctic environment, with PFOS now being recognized as a global POP. Because of the potent surfactant properties of these substances, they are generally used at low concentrations in products and the use of them may therefore not always be clearly known. However, a better knowledge on how these substances are used will increase the possibilities to decrease the environmental emissions directly at the sources.

In conclusion, the aim of this study is to find more information on how per- and polyfluorinated substances are used in the Nordic society and to what extent they may be emitted to the Nordic and Arctic environment. These data will be useful in the process of regulating these substances within REACH or by other international forums like the Stockholm Convention. 


\section{Introduction}

The Nordic Chemical Group (NKG), which is subordinate to the Nordic Council of Ministers, has commissioned the authors, through the Climate and Pollution Agency (KLIF), to undertake a survey that aims to present an overview of the most used PFCs in the Nordic countries besides PFOS/PFOA.

This survey contains three stages namely 1) Identification of relevant per- and polyfluorinated substances and their use in different applications on the Nordic market, 2) Potential emissions and exposure of substances in applications identified in stage 1 and, 3) A summary of knowledge on toxicity of the most important and prioritized substances in this survey.

Table 1. Focus categories of per- and polyfluorinated substances (PFC)

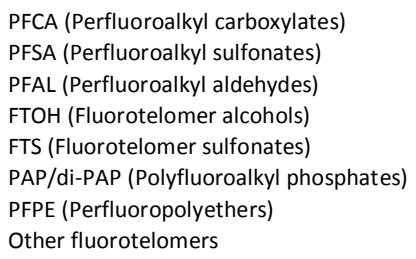

The substances in Table 1 were reviewed concerning their use, occurrence, environmental fate and impact along their life cycle in the Nordic countries (Finland, Sweden, Denmark, Iceland and Norway) including use, exposure and unintentional occurrence in industrial manufacturing and applications and other possible public and industrial sources such as long range transport by air. 



\section{Introduction to fluoro- chemistry}

Polyfluoroalkylated substances (PFCs) belong to a large and complex group of organic substances that are extremely versatile and used in a variety of industrial and household applications.

The main characteristics of the polyfluorinated compounds are the replacement of most hydrogen by fluorine in the aliphatic chain structure. Some of these organic fluorine compounds are known as perfluorinated, which means that all hydrogens have been replaced with fluorine. PFCs are synthetically produced compounds which do not occur naturally, and have been manufactured for 50 years (Kissa, 2001).

An understanding of the chemistry of fluorinated surfactants must consider three distinct structural aspects, namely the hydrophobic/oleophobic "tail" that contains a high proportion of fluorine, the hydrophilic group, and the "spacer" organic group linking these two portions of the surfactant together. As with hydrocarbon surfactants, the important fluorinated surfactants include a diverse range of hydrophilic groups:

- Anionic (e.g. sulfonates, sulfates, carboxylates, and phosphates).

- Cationic (e.g. quaternary ammonium).

- Nonionic (e.g. polyethylene glycols, acrylamide oligomers).

- Amphoteric (e.g. betaines and sulfobetaines).

The practical and commercial range of the hydrophobic/oleophobic "tail" of the fluorinated surfactant is limited. Perfluoroalkyl $\left(\mathrm{F}\left(\mathrm{CF}_{2}\right)_{\mathrm{n}}-\right.$ or $\left.\mathrm{RF}-\right)$, or perfluoropolyether $\left((\mathrm{RFO})_{\mathrm{n}}(\mathrm{RFO})_{\mathrm{m}^{-}}\right)$groups are the hydrophobic/ oleophobic portion of most commercially available fluorinated surfactants. Perfluoroalkyl-containing fluorinated surfactants generally originate from either electrochemical fluorination (ECF) with hydrogen fluoride (HF) or telomerisation of tetrafluoroethylene (TFE). Perfluoropolyether-based fluorinated surfactants typically originate from either oligomerisation of hexafluoropropene oxide (HFPO), photooxidation of TFE or hexafluoropropene (HFP), or oligomerisation of fluorinated oxetanes. 


\subsection{Production of fluoro-chemicals}

There are two main production processes for PFCs; electrochemical fluorination (ECF) and telomerisation. In the electrochemical fluorination process, a technical mixture of hydrocarbons (different carbon chain lengths including branched isomers) with a functional group is subjected to fluorination, leading to a mixture of perfluorinated products with the same homologue and isomer pattern. Telomerisation involves coupling tetrafluoroethene, which leads to straight-chained products with an even number of carbon atoms. Fluorotelomer products often possess two carbon atoms adjacent to the functional group which are not fluorinated that yields linear, even carbon number substances. Telomers are produced and used commercially as mixtures, in which the typical length of the chains is between four and eighteen carbon atoms. Fluoro-compounds can be further reacted and will then occur in other chemical compounds, e.g. acrylate polymers. This means that perfluorinated compounds and fluorinated telomers may occur in a large number of different chemical compounds either added as final treatments, impurities and unreacted monomers of the production process or chemically bound to the polymeric structure (Knepper et al., 2011).

\subsubsection{Electrochemical fluorination}

The ECF of organic compounds using anhydrous HF was the first significant commercial process for manufacturing ECF-based fluorinated surfactants. Typically, a hydrocarbon sulfonyl fluoride $\left(\mathrm{R}-\mathrm{SO}_{2} \mathrm{~F}\right.$, for example, $\mathrm{C}_{4} \mathrm{H}_{9} \mathrm{SO}_{2} \mathrm{~F}$ or $\mathrm{C}_{8} \mathrm{H}_{17} \mathrm{SO}_{2} \mathrm{~F}$ ) is transformed into the corresponding perfluoroalkyl sulfonyl fluoride (Rf- $\mathrm{SO}_{2} \mathrm{~F}$, for example, $\mathrm{C}_{4} \mathrm{~F}_{9} \mathrm{SO}_{2} \mathrm{~F}$ or $\mathrm{C}_{8} \mathrm{~F}_{17} \mathrm{SO}_{2} \mathrm{~F}$ ).

The perfluoroalkylsulfonyl fluoride is the fundamental raw material which is further processed to yield fluorinated surfactants. Commercially relevant perfluoroalkylsulfonyl fluorides are derived from 4, 6, 8, and 10 carbon starting materials yielding perfluorobutanesulfonyl fluoride (PBSF), perfluorohexane sulfonyl fluoride (PHxSF), perfluorooctane sulfonyl fluoride (POSF), and perfluorodecane sulfonyl fluoride (PDSF), respectively.

In the ECF process, fragmentation and rearrangement of the carbon skeleton occurs and significant amounts of cleaved, branched, and cyclic structures are formed resulting in a complex mixture of fluorinated materials of varying perfluoroalkyl carbon chain length and branching as well as trace levels of perfluorocarboxylic acid impurities. The most 
basic surfactant derived from the perfluoroalkyl sulfonyl fluoride raw material is the corresponding sulfonate, $\mathrm{RFSO}_{3}$.

Perfluorooctane sulfonate (PFOS) has historically been made in the largest amounts. Perfluorohexane sulfonate (PFHxS) and perfluorodecane sulfonate (PFDS) are also commercially relevant. Recently, the major historic manufacturer of long-chain perfluoroalkyl sulfonyl chemistry, including PHxSF, POSF, and PDSF, ceased their production and moved to the manufacture of PBSF-based fluorinated surfactants (e.g., $\mathrm{C}_{4} \mathrm{~F}_{9} \mathrm{SO}_{2}-\mathrm{R}$ ) which are growing in commercial use (Knepper et al., 2011).

Figure 1. Synthesis of ECF-based fluorinated surfactants (Knepper et al., 2011)

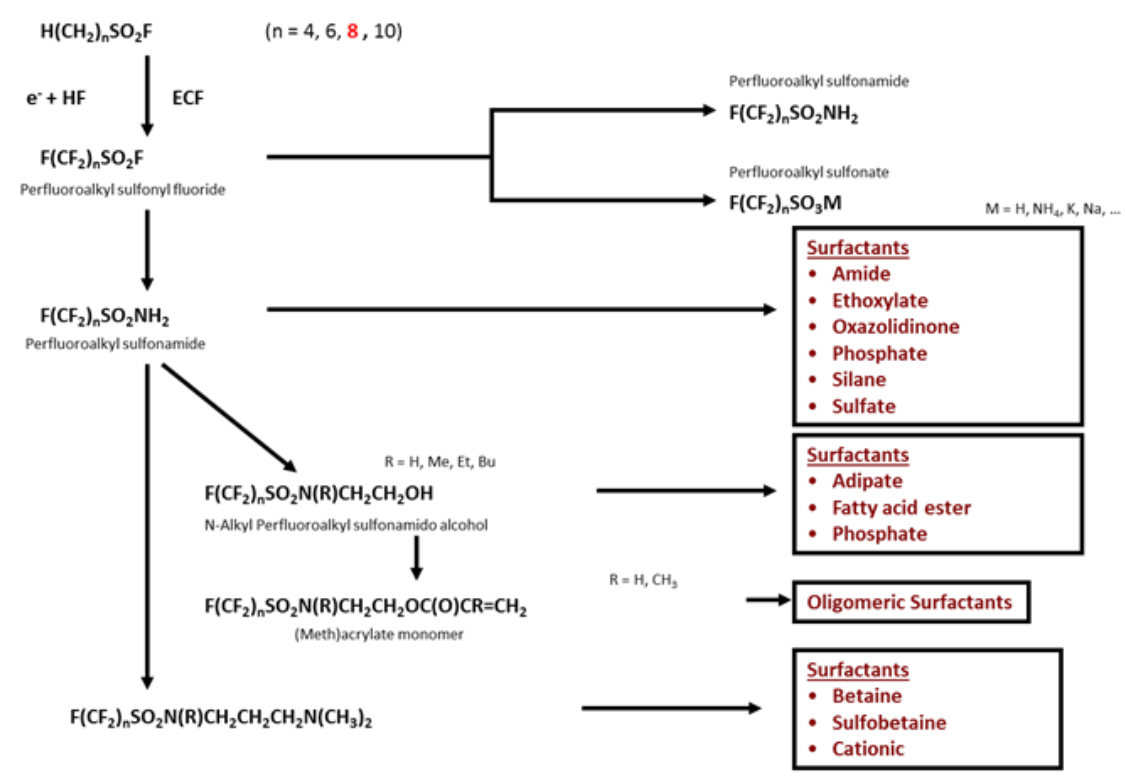

Note: $\mathrm{n}=8$ is PFOS and related substances.

By using the perfluoroalkyl sulfonyl fluoride, for example PBSF, as a basic building block, different products are created through the sulfonyl moiety using conventional hydrocarbon reactions. Perhaps the most versatile intermediates from the ECF process are those containing the perfluoroalkyl sulfonamido functionality, $\mathrm{RFSO}_{2} \mathrm{~N}(\mathrm{R})$-. For example, $\mathrm{C}_{4} \mathrm{~F}_{9} \mathrm{SO}_{2} \mathrm{~N}(\mathrm{CH} 3) \mathrm{CH}_{2} \mathrm{CH}_{2} \mathrm{OH}$, n-methyl perfluorobutylsulfonamido ethanol (MeFBSE).

These primary alcohols can readily be functionalized into fluorinated ethoxylates, phosphates, sulfates, and (meth)acrylate monomers. Fluorinated (meth)acrylates undergo free-radical polymerizations to give oligomeric fluorinated surfactants. In addition, perfluoroalkyl carboxylic acids (PFCAs) and their derivatives have also been synthesized using the 
ECF process. Typically, an alkyl carbonyl fluoride (for example $\mathrm{C}_{7} \mathrm{H}_{15} \mathrm{COF}$ ) is transformed into the corresponding perfluoroalkylcarbonyl fluoride (for example $\mathrm{C}_{7} \mathrm{~F}_{15} \mathrm{COF}$ ). The carbonyl fluoride is then reacted to yield esters, amides, or carboxylic acid salts which have all been commercially produced and used as surfactants. The most widely known is the ammonium salt of perfluorooctanoic acid $\left(\mathrm{C}_{7} \mathrm{~F}_{15} \mathrm{COOH} \cdot \mathrm{NH}_{3}\right)$, whose major historical use has been as a processing aid in the manufacture of fluoropolymers.

\subsubsection{Telomerisation}

The free-radical addition of tetrafluoroethylene (TFE) to pentafluoroethyl iodide yields a mixture of perfluoroalkyl iodides with evennumbered fluorinated carbon chains. This is the process used to commercially manufacture the initial raw material for the "fluorotelomer"based family of fluorinated substances. Telomerisation may also be used to make terminal "iso-" or methyl branched and/or odd number fluorinated carbon perfluoroalkyl iodides as well.

The process of TFE- telomerisation can be manipulated by controlling the process variables, reactant ratios, catalysts, etc. to obtain the desired mixture of perfluoroalkyl iodides, which can be further purified by distillation. While perfluoroalkyl iodides can be directly hydrolysed to perfluoroalkyl carboxylate salts the addition of ethylene, gives a more versatile synthesis intermediate, fluorotelomer iodides. These primary alkyl iodides can be transformed to alcohols, sulfonyl chlorides, olefins, thiols, (meth) acrylates, and from these into many types of fluorinated surfactants. The fluorotelomer-based fluorinated surfactants range includes nonionics, anionics, cationics, amphoterics, and polymeric amphophiles (Knepper et al., 2011). 
Figure 2. Synthesis of fluorotelomer-based fluorinated surfactants, (Knepper et al., 2011)

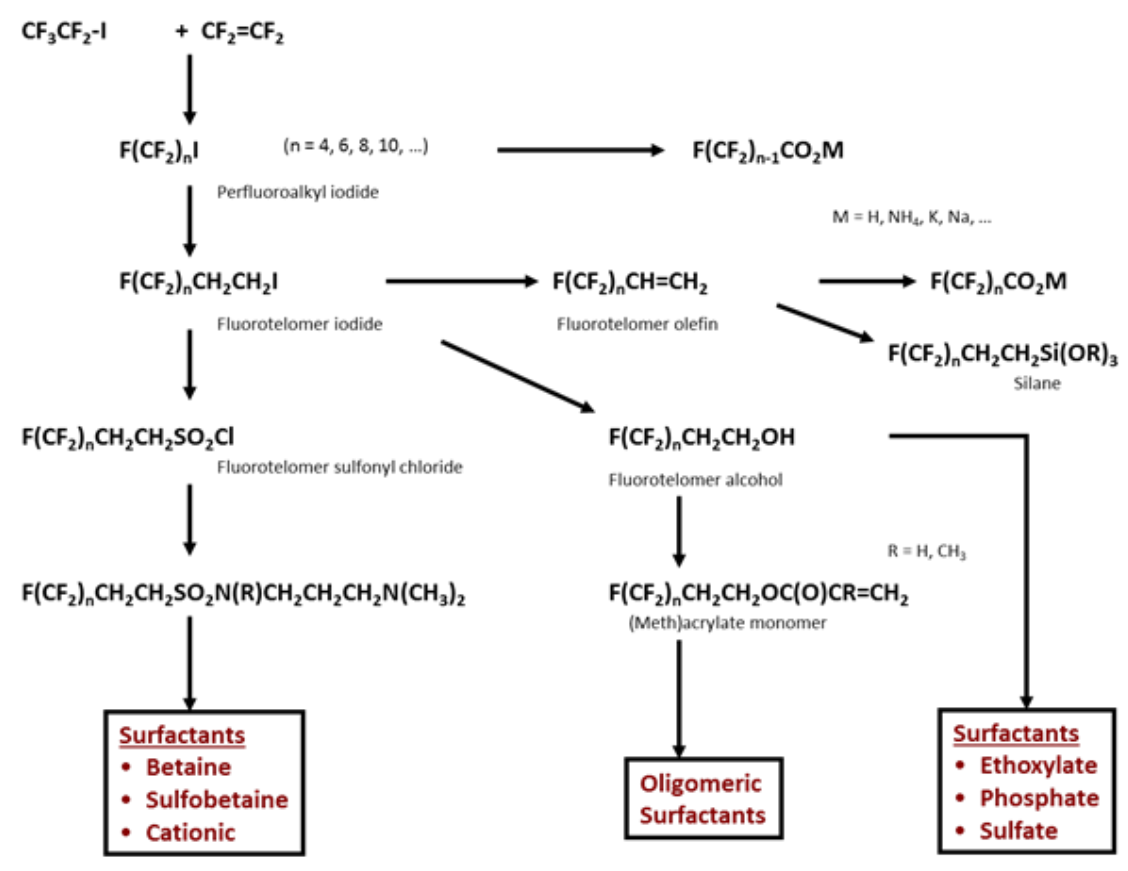

Note: $\mathrm{n}=8$ is PFOA and related substances

\subsection{3 "Per- and Poly- Fluorinated Ethers"}

Per- and polyfluorinated ether-based fluorinated surfactants typically have 1,2, or 3 perfluorinated carbon atoms separated by an ether oxygen, depending on the route to the perfluoropolyether intermediate. The photooxidation of TFE or HFP gives oligomers or polymers with monoor di-acid end groups. These perfluoropolyethers have random sequences of $-\mathrm{CF}_{2} \mathrm{O}$ - and either $-\mathrm{CF}_{2} \mathrm{CF}_{2} \mathrm{O}$ - or $-\mathrm{CF}\left(\mathrm{CF}_{3}\right) \mathrm{CF}_{2} \mathrm{O}$ - units, from TFE or HFP, respectively (Knepper et al., 2011).

In general, the photooxidation of TFE yields mostly difunctional perfluoropolyether acid fluorides, while the photooxidation of HFP yields mostly the monofunctional perfluoropolyether acid fluoride.

The fluoride catalyzed oligomerisation of HFPO, an epoxide, yields a mixture of perfluoropolyether acid fluorides, which can be converted to many types of surfactants, analogous to the fluorinated surfactants from the ECF syntheses. Per- and poly-fluorinated ether surfactants are the newest commercially available substances in this rapidly expanding group of fluorinated surfactants. For example, the phosphate is used as a grease repellent for food contact paper. Per- and polyfluorinated poly- 
ether carboxylates are also used as processing aids in the synthesis of fluoropolymers. Per- and polyfluorinated polyether silanes are used as surface treatments (Knepper et al., 2011), e.g. for stones or as antibiofouling agents for ships.

\subsubsection{Fluorinated oxetanes}

An alternative route to fluorinated surfactants originates from the reaction of polyfluorinated alcohols with oxetanes bearing a $-\mathrm{CH}_{2} \mathrm{Br}$ group in their side-chains to create fluorinated oxetane monomers that undergo ring-opening polymerisation to give side-chain polyfluorinated polyethers. Oxetane-based fluorinated surfactants are offered in many forms and functionalities, such as phosphates and ethoxylates (Knepper et al., 2011). 


\section{Methodology and limitations}

This chapter gives an overview of how the investigation is carried out as a whole and how the three stages 1) Identification of relevant per- and polyfluorinated substances and their use in different applications on the Nordic market, 2) Potential emissions to and occurence in the Nordic environment of the substances described in stage 1, and 3) A summary of knowledge on toxicity of the most important and prioritized substances in this survey, are linked to each other.

\subsection{Methodology}

This project is aiming to seek information about uses of less discussed per- and polyfluorocompounds beside PFOA and PFOS. In order to evaluate uses, occurrence and finally toxicity of some prioritised substances the project was structured and performed in three stages, namely:

- Stage 1 - Identification of relevant per-and polyfluorinated substances and their use in different applications on the Nordic market In stage 1 the following were carried out: a) establishing a database of poly- and perfluorinated substances that may be used on the Nordic market by extraction of a net list which is based on three other lists: A list from OECD, the REACH preregistration database and the Nordic SPIN database and $b$ ) a mapping of Nordic market information through a questionnaire to more than 50 market actors in the Nordic market within the following sectors:

- Aviation hydraulic fluids .

$\circ$ Fire fighting foams .

○ Pesticides.

- Metal plating (hard metal plating and decorative plating).

- Electronic equipment and components.

- Chemically driven oil and mining production.

- Carpets, leather and apparel, textiles and upholstery.

○ Paper and packaging. 
- Coating and coating additives.

- Construction products.

- Medical and healthcare products.

- Stage 2 - Occurence of per- and polyfluorinated substances Identified poly- and perfluorinated substances from stage 1, both from the net list practice and/or answers from Nordic market were meant to be further studied concerning their occurrence in industrial and consumer products, in environment and humans. However, the results from stage 1 did not really give a basis to perform stage 2 . Stage 2 was therefore carried out by compiling the occurrence data for per- and polyfluorinated substances that could be found in literature. Findings from this stage resulted in a priority list of the most frequently occurring groups of PFCs in the Nordic environment and in humans which summarises our current knowledge. This priority list - for the stage 3 work - was prepared in consultation with KLIF/NORAP.

- Stage 3 - Toxic effects of per-and polyfluorinated substances on humans and the environment

The priority list from stage 2 was elaborated in ranking order to describe known toxicity data from publicly available literature sources to support future possible regulatory measures from the Nordic authorities.

\subsection{Limitations}

One major and primary limitation in the intial mapping study is the lack of reliable specific substance data from the market due to the lack of both substance identification and trade secrets. Therefore only publicly available information sources are applied.

There is a major focus of PFCs in the Nordic environment in this survey, consequently literature sources used relate to environmental compartments in the Nordic environment, including in the Arctic.

However, there are limitations in the monitoring data as well, since only PFCs with commercially available analytical reference substances can be analysed and identified in the various studies.

Since there is a strong progress in research in this field especially over the last few years there may be a few very recent publications (also currently unpublished) that have by necessity been left out due to the timing of this survey. 


\section{Mapping of use of per- and polyfluorinated substances on the Nordic market}

The mapping of the use of per- and polyfluorinated substances on the

Nordic market was carried out by use of the following instruments:

- Producing a "net list" of PFCs in use on the Nordic market by use of public available lists of PFCs in use.

- Contacting a selection of producers, suppliers and users of PFCs on the European and Nordic market.

- Using information in literature and knowledge from the institutions and persons performing this study.

The first two steps are described in more detail below.

\section{1 "Net list" of PFCs in use on the Nordic market}

An extraction of a "net list" of PFCs in use in the Nordic countries was performed by use of databases available on the Nordic/European market. There are mainly three lists of PFCs publicly available:

- OECD list from 2007. ${ }^{1}$ This list covers substances and polymers that were used on the global market at that time. It is not considered to be up-to-date.

- REACH Pre-registration database. ${ }^{2}$ This list covers phase-in ${ }^{3}$ substances and polymers intended to be registered under REACH

\footnotetext{
${ }^{1}$ Lists of PFOS, PFAS, PFOA, PFCA, Related Compounds and Chemicals that may degrade to PFCA (as revised in 2007). Organisation for Economic Co-operation and Development, 21 August 2007. ENV/JM/MONO(2006)15.

${ }^{2} \mathrm{http} / / /$ echa.europa.eu/information-on-chemicals/pre-registered-substances

${ }^{3}$ Definition according to REACH Article 3.20)
} 
(i.e. substances manufactured or imported (and/or used) in the EU that are covered by Article 23 concerning transitional provisions).

- SPIN database ${ }^{4}$ that covers per- and polyfluorinated substances and polymers contained in dangerous chemical mixtures used in the Nordic countries. The data has its origin in the national product registries.

Initially, PFOS and PFOA and their related substances ( $\mathrm{C}_{8}$-chemistry) have been excluded in the mapping practice of these lists. Other nonPFOS/PFOA substances and additionally polymers have been matched between the lists in order to get a net list of common per- and polyfluorinated substances and polymers, that may be used on the Nordic market. It is important to emphasise that neither of these lists are complete, often due to company trade secrets, but they may provide a selection of categories of per- and polyfluorinated substances and polymers that may be used in the Nordic market.

The next step in the practice of these three lists mentioned above was to extract the common per- and polyfluorinated substances and polymers on each list to receive a "net list" of substances and polymers that are used in EU and the Nordic countries respectively.

A combination of the OECD list and the REACH pre-registration database (and excluding PFOS and PFOA and related substances) resulted in the so-called "European net list" of substances that were on the OECD list and were pre-registered in the REACH system. The "European net list" consisted of 518 substances, i.e. 518 PFCs may be in use on the European market. Of these 79 were polymers or not-precisely defined mixtures which are listed at the end.

A combination of this "European net list" and the Nordic SPIN database resulted in a so-called "Nordic net list" of 118 substances, i.e. 118 PFCs may be in use on the Nordic market. Of these 27 were polymers or not-precisely defined mixtures, which are excluded from the schemes but listed at the end. 91 CAS numbers were therefore included in the sorting as the final "Nordic net list (excluding polymers or not precisely defined mixtures)". We conclude that these PFCs for which there is publicly available information may be used on the Nordic market.

Since neither of these databases contains complete information on the market use of PFCs, the net list is necessarily incomplete and there

${ }^{4}$ http://www.spin2000.net/ 
may be other PFCs used on the Nordic market in addition to those found in the net list.

A more detailed categorization of the pre-registered 518 nonPFOS/PFOA PFCs in REACH (the "European net list") is found in Appendix B. This includes the polyfluorinated substances that potentially can be used on the Nordic market.

Table 2. The $\mathbf{3 5}$ categories of PFCs that were identified in the "net list" exercise

\begin{tabular}{|c|c|}
\hline Identified PFC categories & Possible fluoro process \\
\hline Perfluoroalkane sulfonic acids (PFASs) & ECF \\
\hline Perfluoroalkane sulfonates (salts) & ECF \\
\hline Perfluoroalkane sulfinic acid/sulfinates & ECF \\
\hline Perfluorocycloalkane sulfonic acid and derivatives & ECF \\
\hline Perfluoroalkane sulfonamides (FASAs) & ECF \\
\hline Perfluoroalkane sulfonamide, quaternary ammonium salts & ECF \\
\hline Perfluoroalkanesulfonamide acrylates (MeFASACs) & ECF \\
\hline Perfluoroalkane sulfonamide methacrylates & ECF \\
\hline Perfluoroalkane sulfonamide phosphates & ECF \\
\hline Perfluoroalkane sulfonyl halides & EFC \\
\hline Other polyfluoroalkyl sulfur compounds & ECF \\
\hline Perfluoroalkyl carboxylic acids (PFCA) & Telomerisation \\
\hline Perfluoroalkyl carboxylic salts & Telomerisation \\
\hline Perfluoroalkyl alcohols/ketones & Telomerisation \\
\hline Perfluoroalkyl carboxylic acid halides & Telomerisation \\
\hline Perfluoroalkyl halides & Telomerisation \\
\hline Perfluoroalkyl alkyl ethers & Telomerisation \\
\hline Perfluoroalkyl amines & Telomerisation \\
\hline Perfluoroalkyl amino acids/salts/esters & Telomerisation \\
\hline Perfluoroalkyl phosphates & Telomerisation \\
\hline Perfluoroalkyl acrylates & Telomerisation \\
\hline Perfluoroalkyl methacrylates & Telomerisation \\
\hline Other perfluoroalkyl carboxylic esters & Telomerisation \\
\hline Perfluoroalkyl heterocyclic compounds & Telomerisation \\
\hline Perfluoroalkyl silanes & Telomerisation \\
\hline Fluorotelomer alcohols & Telomerisation \\
\hline Fluorotelomer halogenides & Telomerisation \\
\hline Fluorotelomer sulfonates, sulfonyl chlorides and sulfonamides & Telomerisation \\
\hline Fluorotelomer acrylates & Telomerisation \\
\hline Fluorotelomer methacrylates & Telomerisation \\
\hline Other acrylates & Telomerisation \\
\hline Fluorotelomer phosphates & Telomerisation \\
\hline Other fluorotelomers & Telomerisation \\
\hline Polymers & No information \\
\hline Undefined mixtures & No information \\
\hline
\end{tabular}

Additionally structure formulas, synonyms, acronyms, trade names, physical-chemical data and use data have been collected. Only a few of these data, however, are included in the tables that were further developed in project phase 2 .

The applied names are as simple as possible and we have chosen to use the most easy to understand. Those are not necessarily the most correct ones, but we have made this choice to make it easier to get an overview and see homologue rows and relationships. That is 
also why the "perfluor" prefix and fluorotelomer names have been used where possible.

\subsubsection{Discussion about the "correctness" of the "net list"}

It must be emphasised that this "Nordic net list" that has been presented in Appendix B only represents some of the "truth". The real picture may very well be very different.

First of all, there is no guarantee that the pre-registered substances are going to be registered in the REACH system. This means that this list may contain substances that may not be used in Europe. On the other hand, new substances were not covered by the transitional provisions and were normally not pre-registered. Therefore the list of the preregistered substances is probably not complete. Finally the substances used for treatment of articles with per- or polyfluorinated substances outside EU are normally not to be registered within the REACH system. Such per- and polyfluorinated substances are therefore not included in the pre-registration list.

Secondly, the SPIN database is only a database of substances used in chemical products (i.e. substances and mixtures) that are classified as dangerous and used (imported or produced) in the Nordic countries. This means that only chemical products that are classified as dangerous are included - thereby excluding chemicals only containing PFCs that are not classified as dangerous. Moreover, the SPIN database does not contain information about articles treated with e.g. per- or polyfluorinated substances such as impregnated textiles.

Finally, the OECD list is from 2007 and may very well not include all per- and polyfluorinated substances in use today.

\subsection{Contacts to producers, suppliers, users and other players on the PFC market}

Based on a search and on the knowledge within the project group, a number of producers, suppliers, users and trade organizations in the different Nordic countries were contacted. Global producers and trade organizations were contacted as well. The main contact was carried out by email. But some of the main players on the market were contacted by phone/interviews. 
Appendix C contains a list of the about 50 companies and organizations that have been contacted in this project. The questionnaire used for the phone/web interviews are also presented in Appendix C.

\subsection{Conclusions}

Parallel with the mapping of the Nordic market extracted net lists (Appendix B) based on a list from OECD, the REACH preregistration database and the Nordic SPIN database, identified 518 per and polyfluorinated substances ("European net list") and 118 per and polyfluorinated substances ("Nordic net list") that might be used on the Nordic market (in blue font in Appendix B). Since neither of these databases contain comprehensive information of per- and polyfluorinated substances, there may be several more per- and polyfluorinated substances that may be used on the Nordic market. These per- and polyfluorinated substances were divided into 35 chemical categories. For these 35 per- and polyfluorinated categories their process origin and possible fate into principal degradation products were estimated for a better understanding of the findings concerning occurrence and impact of per- and polyfluorinated substances in the Nordic environment and to humans. 



\section{Mapping of uses and applications of PFCs on the Nordic market}

The mapping carried out in this project has covered the following uses on the markets of the Nordic countries:

- Aviation hydraulic fluids.

- Fire fighting foams.

- Pesticides (insect baits for control of leaf-cutting ants from Atta spp. and Acromyrmex spp. and insecticides for control of red imported fire ants and termites).

- Metal plating (hard metal plating and decorative plating).

- Electronic equipment and components.

- Chemically driven oil and mining production.

- Carpets, leather and apparel, textiles and upholstery.

- Paper and packaging.

- Coating and coating additives.

- Construction products.

- Medical and healthcare products.

\subsection{Aviation hydraulic fluids}

Alternative hydraulic fluid additives must undergo extensive testing to qualify for use in the aviation industry to sustain severe conditions during use.

In the manufacturing process for aviation hydraulic fluids, a PFOSrelated substance or precursor, such as potassium perfluorooctane sulphonate, was used as an additive to the aviation hydraulic fluids with a 
content of about or less than $0.1 \% \cdot{ }^{5}$ According to the manufacturers, this formulation helps prevent evaporation, fires, and corrosion.

Aviation hydraulic fluids without fluorinated chemicals but based on, for example, phosphate esters are used. These substances can absorb water and the subsequent formation of phosphoric acid can damage metallic parts of the hydraulic system. For this reason, phosphate esterbased hydraulic fluids are routinely examined for acidity as this determines its useful lifetime. Additionally fluorinated chemicals other than PFOS can be used. The potassium salt of perfluoroethylcyclohexyl sulphonate (CAS number. 67584-42-3) ${ }^{6}$ is not a PFOS precursor, and it has been used in hydraulic oils instead of PFOS in the past. However, like other $\mathrm{C}_{6}$ compounds it is likely to be persistent and $3 \mathrm{M}$ which formerly produced this chemical has ceased to do so. A search for other alternatives is said to have been going on for 30 years, starting before PFOS was considered a problematic substance. However it is not possible to get any specific chemical composition of alternatives due to trade secrets. Consequently there is no way to describe their potential feasibility and impact to health and environment in a comprehensive way. ${ }^{7}$

\subsubsection{Identity and properties}

Information gaps

\subsubsection{Type of uses, quantities, producers, downstream users and traders}

There are several trade names and traders on the market. Some are as follows: Arnica, Tellus, Durad, Fyrquel, Houghto-Safe, Hydraunycoil, Lubritherm Enviro-Safe, Pydraul, Quintolubric, Reofos, Reolube, Valvoline Ultramax, Exxon HyJet, and Skydrol. ${ }^{8}$

The fire-resistant aviation hydraulic fluids principally contain trialkyl phosphates, tri-aryl phosphates, and mixtures of alkyl-arylphosphates. However, the products only provide rough descriptions of

\footnotetext{
5 The potassium salt of PFOS was used in such a small quantity that it was not listed on the MSDS at Boeing (Boeing 2001). http://www.boeingsuppliers.com/environmental/TechNotes/TechNotes2001-02.pdf ${ }^{6}$ In the U.S. this chemical is considered a C8 PFOS equivalente and its use in hydraulic fluids is regulated under a Significant New Use Rule: https://www.federalregister.gov/articles/2002/12/09/

02-31011/perfluoroalkyl-sulfonates-significant-new-use-rule 7 UNEP/POPS/POPRC.8/INF/17

${ }^{8} \mathrm{http} / / /$ www.atsdr.cdc.gov/toxprofiles/tp99-c3.pdf
} 
their chemical composition such as "contain phosphate esters". Consequently there are several information gaps concerning the specific chemical composition of each aviation hydraulic fluid but similarly the traders need to know in detail of these oil characteristics since these characteristics are important to aviation security.

Since very little is published concerning the chemical composition of these aviation hydraulic oils there is currently no possibility to assess their environmental and health impact.

There is currently no, scarce or uncertain data available concerning quantities used on the market. ${ }^{9}$

\subsubsection{Efficacy and availability}

There is no available information on cost-effectiveness, efficacy, availability, accessibility and socio-economic considerations.

\subsection{Fire fighting foams}

Fluorinated surfactants are used in fire fighting foams as they are very effective for extinguishing liquid fuel fires at airports, oil refineries etc. Fire fighting foams are divided into:

- Fluoro-protein foams used for hydrocarbon storage tank protection and marine applications.

- Aqueous film-forming foams (AFFF) developed in the 1960s and used for aviation, marine and shallow spill fires.

- Film-forming fluoroprotein foams (FFFP) used for aviation and shallow spill fires.

- Alcohol-resistant aqueous film-forming foams (AR-AFFF), which are multi-purpose foams.

- Alcohol-resistant film-forming fluoroprotein foams (AR-FFFP), which also are multipurpose foams; developed in the 1970s.

\footnotetext{
${ }^{9}$ As aviation hydraulic fluids are essential to the military in Convention member countries they may be a source of information regarding the alternative substances and their quantities used.
} 
PFOS-containing fire fighting foams has a long shelf life (10-20 years or longer) which is why PFOS-containg fire-fighting foams may still be used around the world in accidental oil fires. However, in recent years firefighting foams are not manufactured with PFOS, but with fluorotelomers based on a perfluorohexane $\left(\mathrm{C}_{6}\right)$ chain. However, in China PFOScontaining fire fighting foams are still produced. ${ }^{10}$

\subsubsection{Types of uses, quantities, producers, downstream users and traders}

Information received from the industry during this project confirms that fluorinated surfactants are still used in fire fighting foams. The use of PFOS in fire fighting foams has been discontinued - in new products. However, as PFOS-containing fire fighting foams have a very long shelf life, PFOS-containing fire fighting foams may still be in use globally. EU Regulation from 2008 has, however, ensured that most PFOS stocks have been destroyed. ${ }^{11}$

According to the fire fighting foam industry that has been contacted during this project, the perfluorotelomer used in fire-fighting foams (AFFF, AR-AFFF, FFFP and AR-FFFP) are named $\mathrm{C}_{8}-\mathrm{C}_{20}-\gamma$ - $\omega$-perfluoro telomer thiols with acrylamide (CAS number 70969-47-0) and is used in the most common fluorosurfactants in use in fire-fighting foams since the discontinuation of the PFOS based surfactants. According to the industry most of the manufacturers are committed to continuing use of this chemistry until 2016. ${ }^{12}$

Furthermore, the following summarized information and statements have been received from the fire fighting foam industry about the socalled pure C6 (6:2) fluorotelomers (betaines and aminoxides).

- Production of C6 fluorotelomer in line with the PFOA Stewardship Programme (95\% C6 by 2010, 99.9\% C6 by 2015) has proved challenging with the end product significantly more expensive than the standard C6/C8 mixture.

- It has proved extremely difficult to achieve acceptable operational efficiency for AFFF fire fighting foams - especially as regards burnback resistance - using pure C6 fluorotelomer surfactants.

10 UNEP/POPS/POPRC.6/13/Add.3/Rev.1.

11 UNEP/POPS/POPRC.6/13/Add.3/Rev.1.

12 Personal communication with the fire fighting foam industry/producers in summer 2012. 
- Approximately 20\% more "pure" C6 fluorosurfactant than the older C6/C8 mix is required in order to achieve acceptable performance.

- To date it has proved extremely challenging to formulate an operationally effective fluoroprotein (FP) foam meeting international standards using "pure" C6 fluorotelomer products.

- There are currently very few AFFF manufacturers (one in the Americas, a couple in Europe) whose products are fully C6 compliant and EPA 2015 compliant.

- The majority of manufacturers including a number of major players have taken a conscious decision to stay with the C6/C8 fluorotelomer mixture on grounds of cost and formulation difficulties.

- In particular fluorotelomer surfactants such as CAS number 7096947-0 ( $\mathrm{C}_{8}-\mathrm{C}_{20}-\gamma$ - $\omega$-perfluoro telomer thiols with acrylamide) continue to be used in AFFF formulations with significant potential environmental impact because of the presence of fluorotelomer $\mathrm{N}: 2$ chains with $\mathrm{N}=8$ to $\mathrm{N}=20$; thus degradation products may include PFOA and its even chain long-chain homologues up to C20 - toxicities are claimed to increase with chain length.

- A major feedstock manufacturer will continue therefore to produce the fluorotelomer betaines $1157 \mathrm{~N}$ (the $\mathrm{C} 6 / \mathrm{C} 8$ homologue mix) as well as 1157D containing the purified C6 fluorotelomer (aminoxide containing pure $\mathrm{C} 6$ is also available).

- Of the putative fluorine-free foams on the market relatively few are known to be completely fluorine-free (no organic fluorine present) whereas others are suspected to contain low levels of fluoropolymers.

Within the petroleum industry PFSA (perfluoroalkyl sulfonates) and FTS (fluorotelomer sulfonates) are used (according to the petroleum industry). However, no information about quantity or the specific fluorinated compounds used have been received. ${ }^{13}$

\subsubsection{Efficacy}

Fluorinated surfactants are used within fire fighting because of very good fire fighting properties and because they can be stored for many years under harsh conditions. Furthermore, the fluorinated surfactants

13 Personal communication with the petroleum industry in summer 2012. 
are not too expensive and they are available. ${ }^{14}$ Generally, the fluorinated $\mathrm{C}_{6}$-chemistry used is considered to be effective, however, not as effective as the $\mathrm{C}_{8}$-chemistry, and higher concentrations or amounts may therefore be needed.

\subsubsection{Availability}

The described fluorinated $\mathrm{C}_{6}$-technology are commercially available worldwide and therefore also on the Nordic market.

\subsection{Pesticides}

Pesticides exist as formulations containing active ingredients (the pesticide) and additives (adjuvants) that can help in the application of the pesticide or to enhance the efficiency of the pesticide.

PFCs are used both as active pesticides and as adjuvants in the pesticide formulation.

\subsubsection{Identity and properties}

$N$-Ethyl perfluorooctane sulfonamide (known as sulfluramid or sulfuramid), a PFOS related substance, has been used as an active ingredient in ant baits to control leaf-cutting ants, as well as for control of red imported fire ants, and termites. PFOS and other fluorinated substances have also been used as inert ingredients in pesticides.

There are a number of chemical alternatives to $N$-Ethyl perfluorooctane sulfonamide (known as sulfluramid or sulfuramid), with a multitude of uses: Chlorpyrifos, Cypermethrin, mixture of Chlorpyrifos and Cypermethrin, Fipronil, Imidacloprid, Abamectin, Deltamethrin, Fenitrothion, mixture of Fenitrothion and Deltamethrin but none of these are fluorochemicals.

In addition there are a number of other pesticides which contain one or several fluorine atoms, typically as $-\mathrm{CF}_{3}$ groups.

PFCs adjutants are marketed and patents exist on them, but so far no studies have been conducted on their identity, levels of use or exposure to the environment.

14 Personal information received during this project from a user of fluorinated AFFF's. 


\subsubsection{Types of uses, quantities, producers, downstream users and traders}

PFC adjuvants can have various functions such as being dispersion agents for the pesticide, as a means to better spread the pesticide on leafs/the insect or to increase the uptake through the leafs/insects. PFC adjuvants are typically used in smaller amounts $(0.1 \%)$ than other adjuvant surfactants because they are more effective surfactants. So far there is no overview of producers of these compounds, and it is not known if or to which extent the PFC adjuvants are used in the Nordic countries.

\subsection{Metal plating (hard metal plating and decorative plating)}

Fluorinated surfactants are able to lower the surface tension in chrome acid baths used for chrome plating by forming a thin foamy layer on the surface of the chrome bath. This mist suppressant layer dramatically reduces the formation of chromium-(VI) aerosols $\left(\mathrm{Cr}^{6+}\right)$, which are wellknown as carcinogenic, sensitizing and dangerous for the environment (Poulsen et al., 2011). The challenges to this application are to have a surfactant that are stable in the presence of hot chromic acid and can resist decomposition during the electrolysis as well. Under these demanding conditions perfluorinated surfactants such as PFOS is stable and maintains its activity under a long period.

Previously, PFOS was used for both decorative chrome plating and hard chrome plating processes but new technology applying chromium(III) instead of chromium-(VI) has made PFOS use in decorative chrome plating outdated and unnecessary. For hard chrome plating, however, the process with chromium-(III) does not function. Instead larger closed tanks, or increased ventilation combined with an extraction of chromium-(VI), are suggested as alternative solutions for the applications where a use of chromium-(III) is not possible yet (Poulsen et al., 2011). 


\subsubsection{Identity and properties}

The most common fluorinated surfactant used for hard metal chromium plating has been tetraethyl ammonium heptadecafluorooctane sulfonate (CAS number 56773-42-3; Fluortensid-248), a PFOS-related substance are used in Europe and the Nordic countries ${ }^{15}$ within the metal plating industry. However, in recent years some substitution of PFOS seems to have taken place worldwide with polyfluorinated surfactants instead such as (Poulsen et al., 2011):

- Potassium 1,1,2,2-tetrafluoro-2-(perfluorohexyloxy)ethane sulfonate (CAS number not known) - commercial name F-53 Chromic Fog Inhibitor (Hangzhou Dayangchem Co. Ltd., China).

- Potassium 2-(6-chloro-1,1,2,2,3,3,4,4,5,5,6,6-dodecafluorohexyloxy)1,1,2,2-tetrafluoroethane sulfonate (CAS number not known)). Commercial name F-53B Chromic Fog Inhibitor (Hangzhou Dayangchem Co. Ltd., China).

- $1 H, 1 H, 2 H, 2 H$-Perfluorooctane sulfonic acid/6:2 Fluorotelomer sulfonic acid (CAS number 27619-97-2). Commercial names: Fumetrol ${ }^{\circledR} 21$ (Atotech Skandinavian AB, Sweden) or MiniMist Liquid (MacDermid, USA).

\subsubsection{Types of uses, quantitites, producers, downstream users and traders}

The chromic acid bath that is used for hard chrome plating is extremely reactive and oxidizing, and PFOS is used because it is very resistant to that harsh environment and has an extremely low surface tension. It is very difficult to find another chemical with such useful properties. However, there are PFOS-free fluorinated alternatives on the market based on e.g. fluorotelomers and also fluorine free alternatives as described above, which do not seem to have large market shares today [Poulsen et al., 2011]. In a substitution project for the Danish EPA carried out in 2010 [Poulsen et al., 2011] it was proven that PFOS-free fluorinated alternatives could be used for hard chrome plating instead of PFOS.

Producers and suppliers of mist suppressants for the metal industry have been mapped in (Poulsen et al., 2011).

15 Information received in this project from the contacted suppliers of mist suppressants for the metal plating industry in Europe (Nordic countries). 
- Atotech Skandinavien AB (Sweden).

- EngTech Scandinavia A/S (Denmark).

- Surtec Scandinavia ApS (Denmark).

- Galvano Kemi (Denmark).

- Enthone (Cookson Electronics) (Sweden).

- Kiesow Dr. Brinkmann GmbH (Germany).

- GalvaNord (Elplatek) (Denmark).

- Dr. Günter Dobberschütz (Germany).

- CL Technology GmbH (Germany)

- Schlötter Galvanotechnik (Germany)

- Chembright (China).

- MacDermid Scandinavian (Sweden) Plating Resources, Inc. (USA).

A selection of these companies that in 2009/2010 replied that they delivered to the Nordic market has been contacted to get newer information for this Nordic project. However, replies have not been received from all the companies that participated in the 2009/2010 survey.

In the above mentioned Danish EPA project [Poulsen et al., 2011] it was estimated that the global use of PFOS (calculated as 100\% pure PFOS) was between 32 and 40 tons for the entire metal plating industry based (but with emphasis on non-decorative hard chrome plating) on different information from 2004-2010. The use of pure PFOS in the Nordic countries was estimated to be at least $90 \mathrm{~kg}$ (calculated amounts from contacted suppliers).

Information received by contact to the suppliers of mist suppressants to the Nordic countries in this project shows an actual confirmed use of $3 \mathrm{~kg}$ of pure PFSA (perfluoroalkyl sulfonates) - i.e. tetraethyl ammonium heptadecafluorooctane sulfonate (CAS number 56773-42-3) being sold to the Nordic countries in 2011 as wetting agent for chromium baths (this is only based on information from limited number of suppliers for the Nordic market). Further contact to one hard chromium plater in Denmark confirms that the use of PFOS-based (PFSA) has not changed since the survey carried out in 2009/2010 [Poulsen et al., 2011]. The use of PFSA in Denmark can therefore still be estimated to be around $10 \mathrm{~kg}$ annually. Based on the limited replies from suppliers of mist suppressants to the Nordic countries in this project it is estimated that the total use of PFSA in the Nordic countries is $90 \mathrm{~kg}$ or less as estimated in the 2009/2010 survey. Further concerning brands see Appendix D. 


\subsubsection{Efficacy}

The performance of the non-PFOS fume suppressant is considered as not equal to that of the PFOS based fume suppressants. To achieve the same reduction in surface tension, more products may be necessary and it may have to be replenished more frequently. The project funded by the Danish EPA about substitution of PFOS in non-decorative hard chrome plating (Poulsen et al., 2011) showed that non-PFOS fume suppressant can be used. However, more fume suppressants may be necessary thus enhancing the costs.

\subsubsection{Availability}

Alternatives to PFOS-based mist suppressants are available and to some extent in use in the Nordic countries. The primary alternative identified in the Nordic countries is:

CAS number 27619-97-2: 1H,1H, 2H,2H perfluorooctane sulfonic acid commercial name Fumetrol ${ }^{\circledR} 21$ (Atotech Skandinavian AB, Sweden)

Other commercial alternatives are available as well, but there is not information about the exact identification of the fluorinated surfactant used. Similarly some non-fluorinated alternatives have been introduced as well, but no information of the chemical identification is available (these alternatives are not discussed any further here) (Poulsen et al., 2011).

\subsection{Electronic equipment and components}

Electrical and electronic equipment often requires several parts and processes. PFOS and related chemicals are used in the manufacturing of printers, scanners and similar products. The PFOS-related substances are process chemicals, and the final products are mostly PFOS-free. PFOS have many different uses in the electronic industry and is involved in a large part of the production processes needed for electric and electronic parts that include both open and closed loop processes. Open processes are applied for solder, adhesives and paints. Closed loop processes mostly include etching, dispersions, desmear, surface treatments, photolithography and photomicrolithography.

PFOS can be used as a surfactant in etching processes in the manufacture of compound in semiconductors and ceramic filters. PFOS are then added as part of an etching agent, and rinsed out during the subsequent washing treatment. Desmear process smoothes the surface of a through- 
hole in printed circuit boards. PFOS can be used as a surfactant in desmear agent, i.e. etching agent. PFOS is added in a desmear agent, and rinsed out during washing treatment.

According to information from OECD survey (2006) less than 1 tonne of $N$-ethyl- $N$-[3-(trimethoxysilyl)propyl] perfluorooctane sulfonamide (CAS number 61660-12-6), a PFOS related substance, had been used as an additive in toner and printing inks. Low volumes of PFOS-related substances were also used in sealants and adhesive products. ${ }^{16}$

\subsubsection{Identity, properties, types of uses, producers, downstream users and traders}

Information gaps.

\subsection{Chemically driven oil and mining production}

It is reported that PFOS is used in some parts of the world as surfactants in oil well stimulation to recover oil trapped in small pores between rock particles. Oil well stimulation is in general a variety of operations performed on a well to improve the wells productivity. The main two types of operations are acidization matrix and hydraulic fracturing.

Alternatives to PFOS are PFBS, fluorotelomer-based fluorosurfactants, perfluoroalkyl-substituted amines, acids, amino acids, and thioether acids. In most parts of the world where oil exploration and production is taking place, oil service companies engaged in provision of well stimulation services predominantly use a formulation of alcohols, alkyl phenols, ethers, aromatic hydrocarbons, inorganic salts, methylated alcohols, alipathic fluorocarbons for oil well stimulation. Oil well stimulation services also involve corrosion control, water blocks/blockage control, iron control, clay control, paraffin wax and asphaltene removal and prevention of fluid loss and diverting.

\subsubsection{Identity, properties, types of uses, producers, downstream users and traders}

Information gaps.

16 UNEP/POPS/POPRC.8/INF/17. 


\subsection{Carpets, leather and apparel, textiles and upholstery}

Fluorinated finishes are a technology known to deliver durable and effective oil and water repellence and stain and oil release properties. Historically, fluorinated polymers based on perfluorooctane sulfonyl (PFOS) electrochemical fluorination chemistry have been used. PFOS was not directly used to treat textiles but used to be present at up to $2 \mathrm{wt} \%$ in products. In addition, fluorotelomer-based polymers have also been used.

A restriction of use of PFOS in textiles was introduced within EU legislation in 2008. As in other areas there is no longer a use of $\mathrm{C}_{8^{-}}$ chemistry, but has been replaced by $\mathrm{C}_{6}$-chemistry. ${ }^{17}$

Fluorotelomer alcohols, when used for waterproof and dirt-repellent finishes, are supposed to ensure that PFC degradation products such as PFOS are formed. FTOHs were found in eight of the 14 samples. The highest concentration of fluorotelomer alcohols was $464 \mu \mathrm{g} / \mathrm{m}^{2}$. Test results showed that some manufacturers are already using $\mathrm{C} 6$ telomer alcohols (i.e. $352 \mu \mathrm{g} / \mathrm{m}^{2}$ of 6:2 FTOH). Long-chain C10 telomers were also used in the products $\left(10: 2\right.$ around $\left.200 \mu \mathrm{g} / \mathrm{m}^{2}\right)$. Next to the fluorotelomer alcohols, fluorotelomer acrylates (FTAs), also known as polyfluorinated acrylates, were also detected in some samples (8:2 and 6:2). These acrylates are intermediates in the production of fluorinated polymers. Like the C8 telomers, they can be converted into PFOA through oxidation. No perfluorooctane sulfonate (PFOS) was found in the investigation (Schultze et al., 2006).

\subsubsection{Identity and properties}

Major manufacturers in conjunction with global regulators have agreed to discontinue the manufacture of "long-chain" fluorinated products and move to "short-chain" fluorinated products. Novel short-chain fluorinated products, both short-chain fluorotelomer-based and perfluorobutane sulfonyl-based, have been applied for manufacture, sale and use in carpets, textiles, leather, upholstery, apparel, and paper applications. ${ }^{18}$

\footnotetext{
17 Personal information from the Finnish Textiles and Clothing Industry. 18 UNEP/POPS/POPRC.8/INF/17.
} 


\subsubsection{Types of uses, quantitites, producers, downstream users and traders}

There is currently no publicly available data concerning quantities used on the market. For a selection of trade names, traders and manufacturers, see Appendix D. A Danish survey funded by the Danish EPA estimated that the use of fluorinated substances in impregnated products and impregnation agents (i.e. covering impregnating agents for footwear, carpets, textiles, leather, furniture etc. and impregnated products such as footwear, carpets, clothing, furniture, etc. and other products such as paints, printing inks, ski waxes, floor polish etc.) was between 14 and more than 38 tons of pure fluorinated substances in Denmark. When assumed that the same products and use patterns are applicable to the other Nordic countries, the total amount used within the Nordic countries may be between about 50 tons or more than 100 tons in the Nordic countries.

This former Danish survey as well as contact to the textile industry in the Nordic countries in this survey illustrates that treatment of textiles with fluorinated compounds is not performed in the Nordic countries of any kind of textiles, except maybe in the carpet industry. For brands see Appendix D.

\subsection{Paper and packaging}

Fluorinated surfactants have been evaluated for paper uses since the early 1960s. Perfluorooctyl sulfonamido ethanol-based phosphates were the first substances used to provide grease repellence to food contact papers. Fluorotelomer thiol-based phosphates and polymers followed. Currently polyfluoroalkyl phosphonic acids (PAPs/diPAPs) are used in food-contact paper products and as levelling and wetting agents. Since paper fibers and phosphate-based fluorinated surfactants are both anionic, cationic bridge molecules need to be used in order to ensure the electrostatic adsorption of the surfactant onto the paper fiber. These surfactants are added to paper through the wet end press where cellulosic fibers are mixed with paper additives before entering the paper forming table of a paper machine. This treatment provides excellent coverage of the fiber with the surfactant and results in good folding resistance. An alternative treatment method involves application of a grease repellent at the size press and film press stage which consists of impregnating the formed paper sheet with a surface treatment. Fluorinated phosphate surfactants are not preferred for this mode of paper treatment. In this latter case, fluorinated polymers are used instead of 
surfactants. In terms of oil and water repellency, it is well recognized in the paper industry that phosphate-based fluorinated surfactants provide good oil repellency but have limited water repellency. Acrylate polymers with fluorinated side chains derived from sulfonamido alcohols and fluorotelomer alcohols are the most widely used polymers because they deliver oil, grease, and water repellence. Most recently, perfluoropolyether-based phosphates and polymers have become widely used treatments for food contact paper and paper packaging. ${ }^{19}$

At least one manufacturer has developed a non-chemical alternative for this use. The Norwegian paper producer Nordic Paper is using mechanical processes to produce, without using any persistent chemical, extra-dense paper that inhibits leakage of grease through the paper.

\subsubsection{Types of uses, quantities, producers, downstream users and traders}

See Appendix D

\subsection{Coating and coating additives}

Fluorinated surfactants provide exceptional wetting, leveling and flow control for water-based, solvent-based and high-solids organic polymer coating systems when added in amounts of just 100-500 ppm.

Coating and coating additives include the following uses:

- Cleaning products and polishes.

- Impregnating products.

- Ski waxes.

- Paint and lacquers.

- Dental floss.

Fluorinated surfactants impart various properties to paints and coatings including anti-crater and improved surface appearance, better flow and levelling, reduced foaming, oil repellency, and dirt pickup resistance. They have also been widely used in inks.

${ }^{19}$ UNEP/POPS/POPRC.8/INF/17 
The inclusion of fluorinated surfactants in ink jet compositions has led to better processing through modern printers and excellent image quality on porous or non-porous media. Fluorinated surfactants improved surface wetting during the screen printing of carbon black inks onto Polymer Electrolyte Membrane (PEM) fuel cell electrodes. In addition, fluorinated surfactants improved the cold-water swelling and internal bond strength of wood particleboard bonded with ureaformaldehyde (UF) adhesive resins due to reduced interfacial tension of the resins and improved substrate wetting. ${ }^{20}$

\subsubsection{Type of uses, quantities, producers, downstream users and traders}

The uses of fluorochemicals are quite varied. Specifically, floor polish, where anionic fluorosurfactants are used and at the 100-200 ppm level based on weight of polish.

Performance of most manufacturers of floor polish considers the addition of fluorosurfactants necessary to wet, flow and level properly on a floor.

\section{Paint and lacquers}

According to the Confederation of Danish Industry - Paints \& Lacquers section - there is no use of per- or polyfluorinated substances in the Danish paint industry. ${ }^{21}$ Similarly no use of per- or polyfluorinated substances have been reported in the Finnish Printing Ink industry.

\section{Ski waxes}

The Norwegian National Institute of Occupational Health has in 2009 investigated the exposure of professional users of ski waxes in Norway. This investigation shows that the professional users of ski waxes are exposed to fluorinated chemicals - also airborne. This investigation does not mention the concentration of the fluorinated substances used in ski waxes nor the total amounts used. It is, however, mentioned that ski waxes may contain either a mixture of several perfluoro-n-alkanes $\left(\mathrm{C}_{12}\right.$ $\mathrm{C}_{24}$ ) or perfluoro-n-alkanes $\left(\mathrm{C}_{7}\right.$ or $\left.\mathrm{C}_{8}\right)$ (Daae et al., 2009).

\footnotetext{
20 UNEP/POPS/POPRC.8/INF/17.

${ }^{21}$ Personal communication in the summer of 2012 with the Confederation of Danish Industry.
} 


\subsection{Others}

\subsubsection{Construction products}

According to information received from the industry, the same fluorchemistry that is used in fire-fighting foams (Thiols C8-C20 -gammaomega-perfluoro tellers with acrylomide (CAS 70969-47-0)) is also used in a variety of building and construction products relating to light weight concrete, concrete sandwich panels, and light weight concrete blocks at least in Australia. It is not known whether this use is widespread and in use in the Nordic countries as well. Construction products as the above mentioned are often recycled and crushed and placed in a landfill site. Non-fluorinated alternatives for use in light weight concrete and related concrete products do exist. ${ }^{22}$

\subsubsection{Medical and healthcare products}

According to information received from the Nordic chemical industry within this survey, the following fluorinated compounds have been used and sold in Finland, Denmark and Sweden within processing medical or other healthcare products.

- Tetraethyl ammonium heptadecafluorooctane sulphonate (CAS number 56773-42-3), a PFOS-related substance.

- Tetraethylammonium perfluorobutane sulphonate (CAS number 25628-08-4).

The exact use is not known. Searches on the internet shows that the chemical product can be used for metal chromium plating as well as wetting and flow control agent for coating photographic paper and film. The use was about $150 \mathrm{~kg}$ of pure fluorinated substances in the three above mentioned Nordic countries in 2011.

22 Personal communication with the fire fighting foam industry/producers in summer 2012. 


\subsection{Other important market information for the Nordic market}

3M comments that shorter chain fluorochemical could potentially be used in all application fields as described in the tender document for this project (e.g. metal plating, oil production, carpets, leather, apparel textiles and upholstery, coatings). $3 \mathrm{M}$ is at this moment no longer active in the field of paper \& packaging applications, fire-fighting foams and pesticides. For more details about other alternatives, including shorter chain fluorotelomers (4:2 and 6:2 FTOH) $3 \mathrm{M}$ refers to the manufacturers of this chemistry.

According to information received from the Finnish Plastic Industry, none of their more than 100 member companies are producing fluorinated substances nor importing any. No fluorinated substances are listed in the local buyer's guide of the plastics and rubber industry.

\subsection{Conclusions}

There are considerable information gaps of most per- and polyfluorinated chemicals concerning the exact chemicals composition in commercial products, their quantities produced and uses on the Nordic market. Based on interviews with more than 50 stakeholders with industrial relevance to the Nordic market, this survey has identified two major reasons for these information gaps. Findings show considerable knowledge gaps and/or trade secrets among manufacturers and importers on the Nordic market, whether they trade with articles or chemical products. However it is hard to distinguish to what extent lack of knowledge is predominant compared to trade secrets but both phenomena exist on the Nordic market.

The survey of the use of per- and polyfluorinated substances on the Nordic market has however, revealed the use of a few specific compounds (listed in the table below): 
Table 3. Specific PFCs and their uses in relation to the Nordic market survey and net list practice

\begin{tabular}{|c|c|c|c|}
\hline CAS number & Specific compound used & Use area & Comment \\
\hline 70969-47-0 & $\begin{array}{l}\text { Acrylamide, Thiols, } \mathrm{C}_{8-20} \text {, Gamma, } \\
\text { Omega, Perfluoro, Telomers }\end{array}$ & $\begin{array}{l}\text { Fire fighting foams } \\
\text { Construction products }\end{array}$ & $\begin{array}{l}\text { Not on the } \\
\text { "Nordic net list" }\end{array}$ \\
\hline 161278-39-3 & $\begin{array}{l}\mathrm{C}_{6} \text {-fluorotelomers such as perfluoro- } \\
\text { hexane ethyl sulfonyl betaine, often } \\
\text { used in combination with hydrocar- } \\
\text { bons such as FORAFAC }{ }^{\text {TM }} \text { products } \\
\text { (DuPont) }\end{array}$ & Fire fighting foams & $\begin{array}{l}\text { Not on the } \\
\text { "Nordic net list" }\end{array}$ \\
\hline No information & $\begin{array}{l}\text { Dodecafluoro-2-methylpentan-3-one } \\
(3 \mathrm{M})\end{array}$ & Fire fighting dispenser & No information \\
\hline $56773-42-3$ & $\begin{array}{l}\text { Tetraethyl ammonium heptade- } \\
\text { cafluorooctane sulfonate (Fluorten- } \\
\text { sid-248), a PFOS -related substance } \\
\text { (perfluoroalkyl sulfonate) }\end{array}$ & Fire fighting foams & $\begin{array}{l}\text { Not on the } \\
\text { "Nordic net list" }\end{array}$ \\
\hline 27619-97-2 & $\begin{array}{l}1 \mathrm{H}, 1 \mathrm{H}, 2 \mathrm{H}, 2 \mathrm{H} \text {-Perfluorooctane } \\
\text { sulfonic acid/6:2 Fluorotelomer } \\
\text { sulfonic acid (Fumetrol } 21 \text { or Mini- } \\
\text { Mist Liquid) }\end{array}$ & Metal plating & $\begin{array}{l}\text { Fluorotelomer sul- } \\
\text { fonates - on the } \\
\text { "Nordic net list" }\end{array}$ \\
\hline $61660-12-6$ & $\begin{array}{l}N \text {-ethyl- } N \text {-[3-(trimethoxysilyl)propyl] } \\
\text { perfluorooctane sulfonamide }\end{array}$ & $\begin{array}{l}\text { Electronic equipment } \\
\text { and components }\end{array}$ & $\begin{array}{l}\text { Not on the } \\
\text { "Nordic net list" }\end{array}$ \\
\hline No information & $\begin{array}{l}\text { Fluorotelomer alcohols - e.g. } 6: 2 \text { and } \\
\text { 10:2 FTOH }\end{array}$ & Textiles & $\begin{array}{l}\text { Fluorotelomer alcohols } \\
\text { - on the "Nordic net } \\
\text { list" }\end{array}$ \\
\hline No information & $\begin{array}{l}\text { Polyfluorinated acrylates (FTA 8:2 } \\
\text { and 6:2), methacrylates and fluoro- } \\
\text { acrylate polymers }\end{array}$ & $\begin{array}{l}\text { Textiles and food } \\
\text { contact paper }\end{array}$ & $\begin{array}{l}\text { Perfluoroacrylates - on } \\
\text { the "Nordic net list" }\end{array}$ \\
\hline No information & $\begin{array}{l}\text { Polyflouroalkyl phosphonic acids } \\
\text { (PAPs/diPAPs) }\end{array}$ & Food contact paper & $\begin{array}{l}\text { Fluorotelomer phos- } \\
\text { phates - on the } \\
\text { "net list" }\end{array}$ \\
\hline No information & $\begin{array}{l}\text { Perfluoro-n-alkanes }\left(C_{12}-C_{24}\right) \text { or } \\
\text { perfluoro-n-alkanes }\left(C_{7} \text { or } C_{8}\right)\end{array}$ & Ski waxes & No information \\
\hline
\end{tabular}

It is seen from the table above that the identified chemical compounds with a specific CAS number in general are not available on the "Nordic net list" which implies that these compounds have neither been identified through the REACH pre-registration list and through the SPIN database. This does, however, not mean that they are not used. The chemical groups of compounds for which the survey has identified a use category are in most cases on the "Nordic net list". To conclude: some overlap can be found between the chemical groups of per- and polyfluorinated found in the "Nordic net list" and in this survey of the use in the Nordic countries. Some of the chemical groups that have been identified through chemical analysis for use in specific products are also available on the "Nordic net list" (Appendix B). However, the results of Stage 1 were not 
a good starting point for Stage 2 as the entire area is characterized by a lack of information.

\subsection{Future work}

There is a need to improve access to specific PFC substance information from industrial actors on the market. The current legal tools according to CLP and REACH, such as safety data sheets, provisions regarding registration etc. are not sufficient to provide that information, in particular not for PFCs in articles where almost all production occurs outside the EU. 



\section{Occurrence of per- and polyfluorinated substances}

There are to date considerable data gaps concerning potential emissions into the environment and exposure to humans of the suggested PFCs studied in this survey.

The fate of currently used per- and polyfluorinated substances is in many cases not known. Detection of various final breakdown products in the environment is an indication of ongoing reactions/mechanisms/ activities in areas where non-persistent per- and polyfluorinated substances are used.

In the following chapters the environmental occurrence and the health and environmental effects of the different per- and polyfluorinated substances and their degradation products are described to the extent possible based on the currently available information.

\subsection{Emissions to and occurrence of PFCs into the environment}

PFCs in the Nordic countries have been reported in a number of publications and reports. The current literature covers both biotic and abiotic samples like air, indoor dust, water, wastewater, sludge, sediment and soil.

In the following paragraphs, the existing literature on PFCs is presented, together with the emission estimates for PFOA and PFOS and additional reports of emissions and surface water concentrations of PFOA and PFOS for the whole European territory.

\subsubsection{PFCAs (Perfluoro carboxylates)}

\section{Abiotic and biotic samples}

PFCAs in Nordic countries have been reported in a number of papers and reports. Starting with seawater, PFCAs have been analysed in Greenland, Iceland, Faroe Islands and in Tromsø (Norway). Among PFCAs PFOA has been the most abundant, at concentrations that reached $40 \mathrm{pg} / \mathrm{L}$. PFHxA, PFHpA and PFNA were typically detected at levels of a 
few pg/L (Butt et al., 2010). In a study in Greenland, PFCAs were detected in snow with PFOA being again the dominant compound with concentrations up to $520 \mathrm{pg} / \mathrm{L}$ (Theobald et al., 2007). In Denmark, PFCAs have been analysed and reported for a number of wastewater treatment plants (WWTPs), in the order of a few ng/L. In a few cases, it was reported that concentrations in the effluent wastewaters were slightly higher than the respective in influents (for example, for PFDA). It is interesting to note that there are big variations in the concentrations of PFCAs between WWTPs, but also within the same WWTPs. In particular, in two samples analysed from one WWTP, the concentration of PFOA was below $2 \mathrm{ng} / \mathrm{L}$ in the first sample and $23.5 \mathrm{ng} / \mathrm{L}$ in the second, while the concentration at the effluents was 10.1 and $16.4 \mathrm{ng} / \mathrm{L}$, respectively. In another WWTP samples taken on the same day, contained 4.5 and 6.4 $\mathrm{ng} / \mathrm{L}$ of PFOS in the influent WWTP and 8.7 and $21.0 \mathrm{ng} / \mathrm{L}$ in the effluent (Bossi et al., 2008). The same study reported also sludge concentrations of PFCAs. Only PFOA, PFNA and PFDA were detected, with the latter showing the highest value of $32 \mathrm{ng} / \mathrm{g}$ (dry weight, $\mathrm{dw}$ ). In sewage sludge from Norway, PFUnA, PFTA and PFTrA were detected (Report 2367/2008). Other reports also show findings of PFOA (Report TA3005/2012 and TA 2636/2010). In Iceland and Faroe Islands, PFCAs were regularly below the limit of quantification, and when quantified, their concentrations were normally at $<1 \mathrm{ng} / \mathrm{g}$ (wet weight, ww).

PFCAs were close to the detection limits in marine sediments in Iceland and Faroe Islands (max concentration of $0.09 \mathrm{ng} / \mathrm{g}$ (dry weight) was for PFHxA, Butt et al., 2010), and similarly not detected in background sediments in Norway (Report 2367/2008). However in sediments close to a company that manufactures fire fighting foams the concentrations of PFCAs were particularly high, reaching $326.7 \mathrm{ng} / \mathrm{g}$ for PFuNA. Similarly, PFHxA and PFOA exhibited high concentrations as well, namely 112.9 and $101 \mathrm{ng} / \mathrm{g}$, respectively. The important impact of local sources such as the fire fighting foam used in airports has been proven to contaminate adjacent soils, groundwater and other environmental compartments. In particular, this can be seen in the comparison between background soils close to the major Oslo airports and soils from the airport areas. For background soils, in Rygge and Gardemoen, PFCAs were not detected, whereas soils from the airports exhibited higher concentrations, particularly those from Gardemoen. In the latter, concentration of PFUnDA was $43.6 \mathrm{ng} / \mathrm{g}$, while for PFOA and PFHpA were around 4 ng/g (Klif Report TA-2444/2008). 
In air samples, PFCAs are typically not detected and only PFOA was detected in concentrations that ranged between $0.15-1.51 \mathrm{pg} / \mathrm{m}^{-3}$ in the Norwegian Arctic (Butt et al., 2010).

The occurrence of PFCAs in biotic samples is quite extensively reported. Concentrations in algae, fresh and marine water fish samples, seabirds, pinnipeds, whales, marine mammals, in eggs, plasma and liver have been reported. A lot of these species have been collected from the Arctic. In algae and fish in most cases, PFCAs are below the limit of detection, or when detected, the concentrations are very low. In ice amphipod samples from the Barents sea only PFOA was detected $(3.15 \mathrm{ng} / \mathrm{g}$ ww) (Haukas et al., 2007) and in marine and fresh water fish, PFCAs when detected, was always below 2 ng/g (Haukas et al., 2007, Kallenborn et al., 2004, Bossi et al., 2005). A number of studies have been performed on sea birds. Bossi et al. (2005) did not detect PFCAs in black guillemot and in northern fulmar samples that were collected before 2000. However, Kallenborn et al., 2004 found 0.4 ng/g PFHpA and 1.0$1.3 \mathrm{ng} / \mathrm{g}$ of PFNA in the same sea bird species studied in 2004. Very high concentrations for PFDA and PFDoDA were detected in guillemots by Løfstrand et al. (2008), while PFOA and PFNA were not detected. Concentrations of these PFCs in herring gulls ranging from not detected to $<1 \mathrm{ng} / \mathrm{g}$ were reported by Verreault et al., 2007, however, no temporal trends, meaning no trends over time, could be seen between samples collected in 1993 or 2003. Similarly, no clear temporal trend could be observed for PFNA and PFDA in ringed seals over the last 30 years (Figure 3, Bossi et al., 2005).

Figure 3. Temporal trends of PFCAs in ringed seal samples from two areas in Greenland (Bossi et al., 2005)

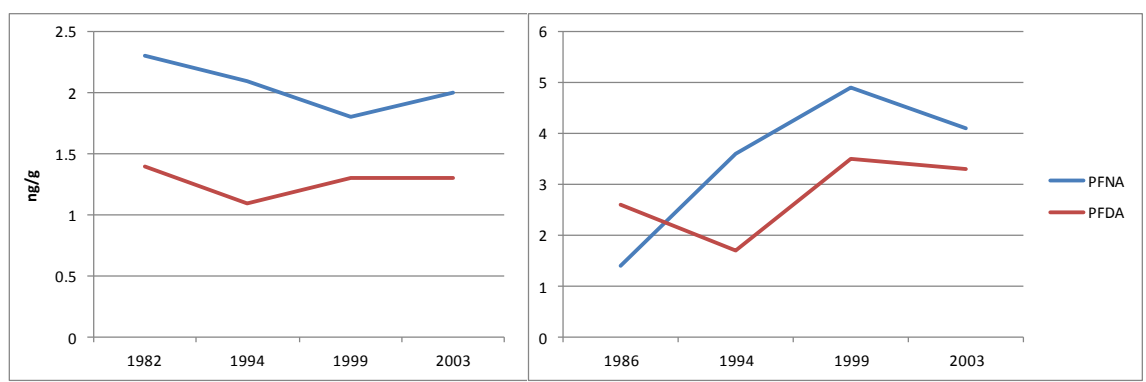

In more recent samples (from 2000 and 2005), Galatius et al., 2011 reported concentrations for harbor porpoises for PFDA and PFUnDA and noticed a small increase between 2000 and 2005. PFDA was the only PFCA that was detected in blue mussels (nd-6.38 ng/g, Klif Report TA- 
2367/2008), whereas PFNA was the dominant PFCA in liver and blood from marine mammals (Smithwick et al., 2005). In a study by Rotander et al., 2012, temporal variations in concentrations of different PFCs were examined in livers of pilot whale, ringed seal, minke whale, harbor porpoise, hooded seal, Atlantic white-sided dolphin and in muscle tissue of fin whales. The sampling spanned over 20 years (1984-2009) and covered a large geographical area of the North Atlantic and West Greenland. In general, the levels of the long-chained PFCAs (C9-C12) increased over the studied period (Rotander, 2012).

\subsubsection{PFSA (Perfluoroalkyl sulfonates)}

\section{Occurence in the environment}

PFSAs have been studied in most of the afore-mentioned studies that reported PFCAs. PFOS is the most important and best studied compound from this class. It is also the one that in almost all cases exhibited the highest concentration levels. In abiotic environmental samples, PFOS was the only PFSA detected in marine sediments in Faroe islands in concentrations just higher than the quantification limit (ND-0.11 ng/g ww, Butt et al., 2010), while PFDS was reported from sediments in Norway in two studies, between which though, there was an important difference in the maximum concentrations ( 0.93 and $0.094 \mathrm{ng} / \mathrm{g}$, by Fjeld et al., 2005 and Bakke et al., 2007, respectively). In sea water and other aquatic samples, PFOS has ranged between ND and $1.18 \mathrm{pg} / \mathrm{L}$, in the Faroe Islands (Butt et al., 2010). The substance was not detected in Iceland, but was found at levels as high as $90 \mathrm{pg} / \mathrm{L}$ in Tromsø, Norway. In the same sample, PFHxS was also detected at the concentration of $16.4 \mathrm{pg} / \mathrm{L}$, being many times higher than in other sea water samples, where it was barely detected. These differences underline the importance of the urban discharges. As a matter of fact, in effluent wastewaters in Denmark, PFOS was detected at concentrations up to $1,115 \mathrm{ng} / \mathrm{L}$ and PFHxS at concentrations up to $19.8 \mathrm{ng} / \mathrm{L}$. Sewage sludge samples have also been analysed for PFSAs and again the prevalence of PFOS and PFHxS was seen. In a Danish WWTP, PFOS concentrations varied between 4.8 and $74.1 \mathrm{ng} / \mathrm{g}$ (dw, Bossi et al., 2008), while in Norway, the range was between 1.2 and $5.16 \mathrm{ng} / \mathrm{g}$ (Report TA 2367/2008). PFDS was also reported in sludge from Norway, at a range of 0.35 and $6.84 \mathrm{ng} / \mathrm{g}(\mathrm{dw})$.

Similarly to what was reported for PFCAs, the importance of local emission sources was assessed by analyzing soils adjacent to airports and remotely from the airports, yet in the same region. The differences in concentrations were 5-10 times for PFOS (40.2 and $226.9 \mathrm{ng} / \mathrm{g}$ in 
soils in Rygge, and from 109.9 to $959 \mathrm{ng} / \mathrm{g} \mathrm{dw}$ in soils close to Gardermoen) and even higher for the other PFSAs that were not detected in background soils (Report 2444/2008). Close to the training station of the company manufacturing fire-fighting foams, the concentrations in stream water was $68,886 \mathrm{ng} / \mathrm{L}$ for PFOS, 37,312 ng/L for PFHxS, which is many orders of magnitude higher than any influent or effluent wastewater sample.

PFOS was the only PFSA detected in the Norwegian Arctic ambient air, however in very low concentrations, close to the limit of quantification $\left(0.02-0.97 \mathrm{pg} / \mathrm{m}^{3}\right.$, Butt et al., 2010). In other air samples from Norway, concentrations of PFDS ranged between nd and $5.13 \mathrm{pg} / \mathrm{m}^{3}$, PFOS ranged between $0.03-3.32 \mathrm{pg} / \mathrm{m}^{3}$ and PFHxS between nd and $0.71 \mathrm{pg} / \mathrm{m}^{3}$.

In biotic samples, again PFOS and PFHxS are the most commonly reported chemicals. PFDS and PFBS are only occasionally detected. Regarding fish samples, PFHxS was only detected in Arctic cod $(0.04 \mathrm{ng} / \mathrm{g}$ ww, Haukas et al., 2007), whereas PFOS was detected in Arctic cod, in dab and shorthorp sculpin, in concentrations that ranged between nd and $28 \mathrm{ng} / \mathrm{g}$, ww (highest concentration in long-rough dab, Kallenborn et al., 2004). PFDS was also detected in fish samples, with its highest concentration (11.6 ng/g ww) observed in long-rough dab as well (Kallenborn et al., 2004). In sea birds, PFOS exhibited its highest concentrations of $134 \mathrm{ng} / \mathrm{g}(\mathrm{ww})$ in glaucous gull samples from the Norwegian Arctic (Verreault et al., 2005), 3-4 times higher than in Herring gull from Hornøya or from Røst.Whereas for PFHxS, the levels were similar in all kinds of sea birds, without any visible trend. PFBS was not detected and PFDS was detected in Herring Gull, but always in very low concentrations (average between 0.04 and $0.21 \mathrm{ng} / \mathrm{g}$, ww). The concentrations of PFOS in ringed seal and whales are broadly in the same range as in sea birds, however, there seems to be important spatial differences. As can be seen in Figure 3, in 1999 and 2003, the differences in PFOS concentrations in ringed seals between West and East Greenland are notable, both in terms of concentration levels, but also as temporal trends. 
Figure 4. Temporal and spatial differences in the concentration of PFOS in ringed seals in West and East Greenland (Bossi et al., 2005)

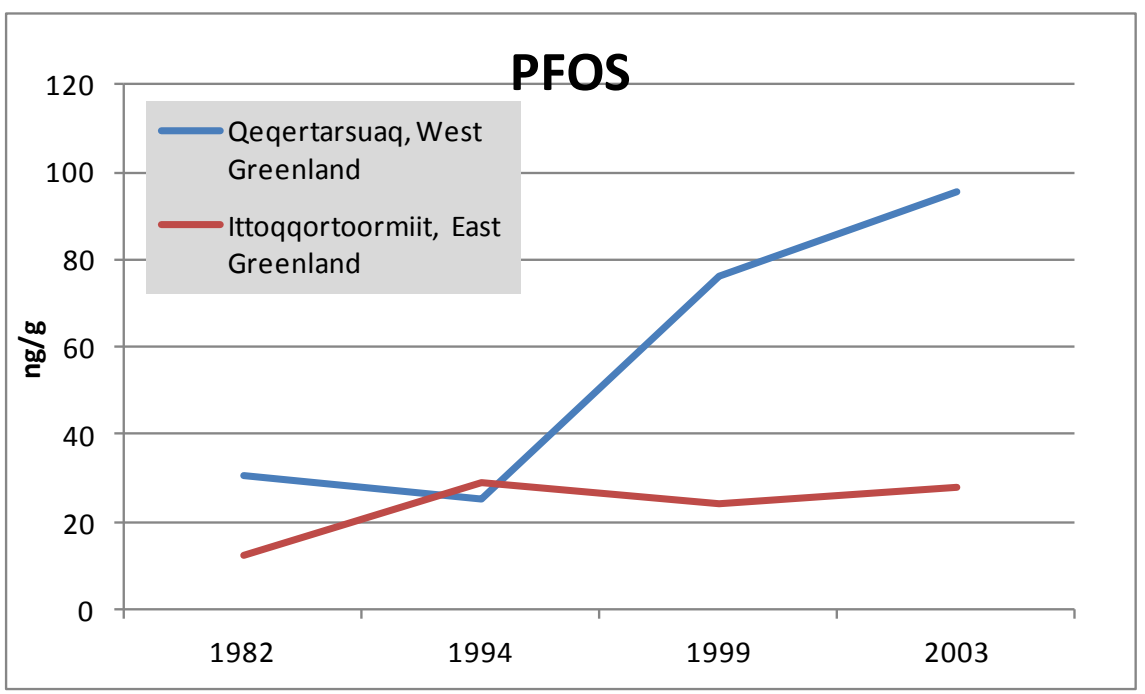

Finally, it should be noted that the highest concentrations for PFOS and PFHxS were reported in polar bears' blood from Svalbard (Smithwick et al., 2005). In particular, PFHxS exhibited an average value of 2,940 ng/g (ww) and PFOS had an average of 1,290 ng/g (ww). PFOS was particularly high also in the liver samples $(1,170-1,285 \mathrm{ng} / \mathrm{g})$, whereas PFHxS was relatively low (36 ng/g ww).

\subsubsection{PFAL (Perfluoro aldehydes)}

The literature research has shown that there are no published studies dealing with the environmental concentrations of perfluoro aldehydes in the Nordic countries.

\subsubsection{FTOH (fluorotelomer alcohols)}

Fluorotelomer alcohols are fluorotelomers that have one alcohol group. They are characterised by high volatility and in the environment act as precursors to PFCAs. FTOHs were broadly produced and it was estimated that between 2000 and 2002, more than 4,000 tonnes were produced annually. Since 2002 their production decreased sharply, after $3 \mathrm{M}$ ceased their production and use in their products. 


\section{Occurrence in the environment}

As FTOHs are volatile compounds, their environmental occurrence is predominantly in the gas phase in the atmosphere. Only very few studies exist that have studied FTOHs in the Nordic countries, and in particular, just for Norway. Concentrations are summarized in Table 4.

Table 4. Occurrence of fluorotelomer alcohols in the Nordic countries

\begin{tabular}{|c|c|c|c|c|c|c|c|}
\hline Sample & $\begin{array}{r}4: 2 \\
\text { FTOH }\end{array}$ & $\begin{array}{r}6: 2 \\
\text { FTOH }\end{array}$ & $\begin{array}{r}8: 2 \\
\text { FTOH }\end{array}$ & $\begin{array}{r}10: 2 \\
\text { FTOH }\end{array}$ & $\begin{array}{r}12: 2 \\
\text { FTOH }\end{array}$ & Unit & Country \\
\hline Alnabru (Air) ${ }^{1}$ & nd & nd-1,720 & nd-9,470 & nd-3,460 & nd & $\mathrm{pg} / \mathrm{m} 3$ & Norway \\
\hline Birkenes (Air)1 & nd & $\mathrm{Nd}$ & nd & nd & nd & $\mathrm{pg} / \mathrm{m} 3$ & Norway \\
\hline Kjeller (Air) ${ }^{4}$ & nd & 11.7 & 34.4 & 17.2 & & $\mathrm{Pg} / \mathrm{m} 3$ & Norway \\
\hline Indoor air $^{2}$ & 4.8 & 1,492 & 6,438 & 4,088 & & $\mathrm{pg} / \mathrm{m} 3$ & Norway \\
\hline Indoor air, homes ${ }^{3}$ & 21 & 42 & 10,005 & 3,405 & & $\mathrm{pg} / \mathrm{m} 3$ & Norway \\
\hline Indoor air, Office ${ }^{3}$ & 165 & 266 & 3,151 & 1,970 & & $\mathrm{pg} / \mathrm{m} 3$ & Norway \\
\hline Indoor air, homes ${ }^{4}$ & 114 & 2,990 & 3,424 & 3,559 & & $\mathrm{pg} / \mathrm{m} 3$ & Norway \\
\hline Indoor air, Office ${ }^{5}$ & nd & 212 & 637 & 1,279 & & $\mathrm{pg} / \mathrm{m} 3$ & Norway \\
\hline WWTP, influent ${ }^{1}$ & nd & $\mathrm{Nd}$ & nd & nd & nd & $\mathrm{ng} / \mathrm{L}$ & Norway \\
\hline WWTP, effluent ${ }^{1}$ & nd & $\mathrm{Nd}$ & nd & nd & nd & $\mathrm{ng} / \mathrm{L}$ & Norway \\
\hline WWTP, sludge ${ }^{1}$ & nd & nd-0.01 & nd-0.01 & nd-0.02 & nd- 0.03 & $\mathrm{ng} / \mathrm{g} d \mathrm{w}$ & Norway \\
\hline $\begin{array}{l}\text { Sediments (various sites } \\
\text { in Norway) }\end{array}$ & nd & $\mathrm{Nd}$ & nd-0.09 & nd-0.1 & nd-0.6 & $n g / g d w$ & Norway \\
\hline Mussels & nd & $\mathrm{Nd}$ & nd & nd-0.09 & nd & $\mathrm{ng} / \mathrm{g} \mathrm{dw}$ & Norway \\
\hline
\end{tabular}

${ }^{1}$ Report 2367/2008; ${ }^{2}$ Haug et al., 2011; ${ }^{3}$ Huber et al., 2011; ${ }^{4}$ Barber et al., 2007; ${ }^{5}$ Jahnke et al., 2007.

It can be seen that fluorotelomer alcohols are in very low concentrations in effluents, sludge or sediments, however in air, and particularly in the indoor environment, they can occur at very high levels. 8:2 FTOH is the most abundant FTOH with its levels that reach $10,000 \mathrm{pg} / \mathrm{m}^{3}$, followed by 10:2 FTOH. In one case 10:2 FTOH exhibited higher concentrations than 8:2 FTOH. The study of Barber et al. (2007) compared FTOH concentrations between Kjeller and various sites in the United Kingdom and it was shown that the concentrations in Norway were always lower than in the UK, and in particular $\Sigma$ FTOHs in Kjeller $\left(63.3 \mathrm{pg} / \mathrm{m}^{-3}\right)$ were $7-8$ times lower than in Manchester.

\subsubsection{FTS (fluorotelomer sulfonates)}

\section{Occurrence in the environment}

Table 5 presents results from the occurrence of fluorotelomer sulfonates in the Nordic environment. 6:2 FTS is the most studied member and has been detected in soil, sediment, groundwater, indoor dust and in some few cases also in biota. Its' concentrations are relatively low and the high concentrations presented in Table 5 for soil, sediment and groundwater, are from an area that was located close to contaminated sites. In the 
various biota analysed, 6:2 FTS was detected only in ice amphipod, sea snails and in ivory gull eggs.

In the indoor air, 6:2 FTS has been detected in two cases in dusts, in concentrations that ranged between nd and $38.7 \mathrm{ng} / \mathrm{g}$. 8:2 FTS was detected only in indoor air dust in concentrations ranging from nd to $50.2 \mathrm{ng} / \mathrm{g}$.

Table 5. Occurrence of FTSs in the Nordic environment

\begin{tabular}{|c|c|c|c|c|}
\hline & 6:2 FTS & $8: 2$ FTS & Unit & Country \\
\hline Groundwater $^{1}$ & 3,220 & & $\mathrm{ng} / \mathrm{L}$ & Norway \\
\hline Soil $^{1}$ & $1,020,688$ & & $\mathrm{ng} / \mathrm{kg}$ & Norway \\
\hline Sediment $^{1}$ & 1,090 & & $\mathrm{ng} / \mathrm{g} d \mathrm{w}$ & Norway \\
\hline Contaminated stream water ${ }^{1}$ & 9,388 & & $\mathrm{ng} / \mathrm{L}$ & Norway \\
\hline Indoor dust, homes ${ }^{2}$ & nd-38.7 & nd-50.2 & $\mathrm{pg} / \mathrm{g}$ & Norway \\
\hline Indoor air, homes ${ }^{7}$ & nd & & $\mathrm{pg} / \mathrm{m}^{3}$ & Norway \\
\hline Background soil ${ }^{1}$ & nd & & $\mathrm{ng} / \mathrm{g} d \mathrm{w}$ & Norway \\
\hline Sea snail ${ }^{1}$ & $2.4-129$ & & $\mathrm{ng} / \mathrm{g} d \mathrm{w}$ & Norway \\
\hline Indoor Air dust ${ }^{3}$ & 9 & 15 & $\mathrm{ng} / \mathrm{g}$ & Norway \\
\hline Ivory gull egg ${ }^{4}$ & 0.25 & & $\mathrm{ng} / \mathrm{g}$ & Russia \\
\hline Ivory gull egg ${ }^{4}$ & 0.28 & & $\mathrm{ng} / \mathrm{g}$ & Russia \\
\hline Ivory gull egg ${ }^{4}$ & 0.37 & & $\mathrm{ng} / \mathrm{g}$ & Russia \\
\hline Ice amphipod ${ }^{5}$ & 0.48 & & $\mathrm{ng} / \mathrm{g}$ ww & Barents Sea \\
\hline Polar $\operatorname{cod}^{5}$ & nd & & $\mathrm{ng} / \mathrm{g}$ ww & Barents Sea \\
\hline Black guillemot ${ }^{5}$ & nd & & $\mathrm{ng} / \mathrm{g}$ ww & Barents Sea \\
\hline Glaucus gull ${ }^{5}$ & nd & & $\mathrm{ng} / \mathrm{g}$ ww & Barents Sea \\
\hline Influent ${ }^{6}$ & $\mathrm{Nd}$ & nd & $\mathrm{ng} / \mathrm{L}$ & Norway \\
\hline Effluent $^{6}$ & $\mathrm{Nd}$ & nd & $\mathrm{ng} / \mathrm{L}$ & Norway \\
\hline Sludge ${ }^{6}$ & $\mathrm{Nd}$ & nd & $\mathrm{ng} / \mathrm{g}$ ww & Norway \\
\hline Sediment $^{6}$ & nd-2.37 & nd & $\mathrm{ng} / \mathrm{g}$ ww & Norway \\
\hline Air $^{6}$ & nd-0.11 & nd & $\mathrm{pg} / \mathrm{m}^{3}$ & Norway \\
\hline Sediment ${ }^{6}$ & $\mathrm{Nd}$ & nd & $\mathrm{ng} / \mathrm{g}$ ww & Norway \\
\hline Mussel $^{6}$ & $\mathrm{Nd}$ & nd & $\mathrm{ng} / \mathrm{g}$ ww & Norway \\
\hline Cod liver ${ }^{6}$ & $\mathrm{Nd}$ & nd & ng/g ww & Norway \\
\hline
\end{tabular}

${ }^{1}$ Report 2444/2008; ${ }^{2}$ Huber et al., 2011; ${ }^{3}$ Haug et al., 2011; ${ }^{4}$ Miljeteig et al., 2009; ${ }^{5}$ Haukas et al., 2007; ${ }^{6}$ Report 2367/2008; ${ }^{7}$ Barber et al., 2007.

\subsubsection{Other fluorinated telomers}

In 2002 , polymer production consumed $80 \%$ of the fluorotelomers produced worldwide, according to the Telomer Research Programme (2002). A study of carpets treated with various polymeric and surfactant fluorocoatings found residual FTOHs ranging from $0.04-3.8 \%$ of the dry mass of the commercial fluorochemicals. 6:2, 8:2, 10:2 and 12:2 FTOH and NMeFOSE were measured, and the contribution of the FTOHs varied greatly between the products; however 8:2 FTOH was the most predominant compound in the study (Dinglasan-Panlilio and Mabury, 2006). It is common that the FTOHs are linked via esterbonds to a polymer backbone, as in the case of fluoroacrylates. FTOHs can be released to its surroundings if the ester bond is hydrolysed, upon exposure to water, heat and a catalyst, such as acids. 
The literature research has shown only few data about compounds like FTCA, FTUCAS and mainly in air and water samples.

\subsubsection{PAP/di-PAP (polyfluoroalkyl phosphate esters)}

The literature research has shown that there are no published studies dealing with the environmental concentrations of polyfluoroalkyl phosphate esters in the Nordic countries. No studies have so far investigated point pollution sources of PAPs in Nordic countries, and at present it is not known if PAPs are produced in the Nordic countries. PAPs might however be applied as coating agents on paper and board, and can be released to the environment from e.g. paper manufacturing plants or paper conversion industries.

Canadian studies have thus shown that PAPs are present in waste water treatment plant sludge and in paper fibres (D'eon 2009). PAPs have also been found in Danish and Swedish paper and board food packaging (Trier 2011), where 57\% of the samples taken in 2009 from Danish, Swedish and Canadian retailers (Trier 2011, thesis) contained diPAPs in the material (Trier 2011, thesis). It is therefore likely, that also wastewater sludge in Nordic countries contain PAPs, which upon hydrolysis (e.g. catalysed by heat and acidic conditions) release fluorotelomer alcohols. Sludge which are used as fertilizers on fields, and containing PAPs, are thus likely to release FTOHs and PFCAs. Other possible exposure routes of PAPs to the environment are via household waste sites and during storage of recycled paper.

\subsubsection{Other fluorinated compounds of interest}

The data on other fluorinated compounds of interest in the Nordic countries are scarse, almost non existent. This lack of data supports one of the conclusions of this report that screening projects should be further encouraged.

\subsubsection{Emission calculations for fluorinated compounds in the Nordic countries}

The compilation of emission inventories for the various chemicals that occur in the environment is a challenging task that requires detailed information.,Even when much information is available, the final estimates are highly uncertain values. An emission inventory needs to contain accurate information on the emissions directly from materi- 
als/products, or from industrial discharges during manufacturing etc. but also needs to provide information regarding spatial and temporal distribution (Breivik et al., 2007). In other cases, the use of environmental monitoring data is used together with climatologic conditions and other relevant input parameters in order to estimate emissions (Pistocchi and Loos, 2009). As seen from the results presented in the relevant chapters, there are only few data on the occurrence of fluorinated chemicals in the Nordic countries, something that makes the estimate of emissions based on the Pistocchi and Loos method quite difficult (Pistocchi and Loos, 2009).

In the literature, one can find information about the emissions of PFOS and PFOA. Based on the report TA-2354/2007 prepared for the Norwegian Pollution Control Authority (2007), the PFOA estimated emissions (primary and secondary and due to long range transport) for Norway were 130-380 Kg per year. Important primary sources were considered to be carpets $(12 \mathrm{Kg} / \mathrm{y})$, coated and impregnated paper $(1.3$ $\mathrm{Kg} / \mathrm{y}$ ) and textiles $(0.5 \mathrm{Kg} / \mathrm{y})$. Pistocchi and Loos (2009) also estimated PFOA concentrations and emissions for almost the whole European territory, thus Nordic countries were included.

\section{Figure 5. PFOA emission estimates for Europe (Figure taken from Pistocchi and} Loos, 2009)

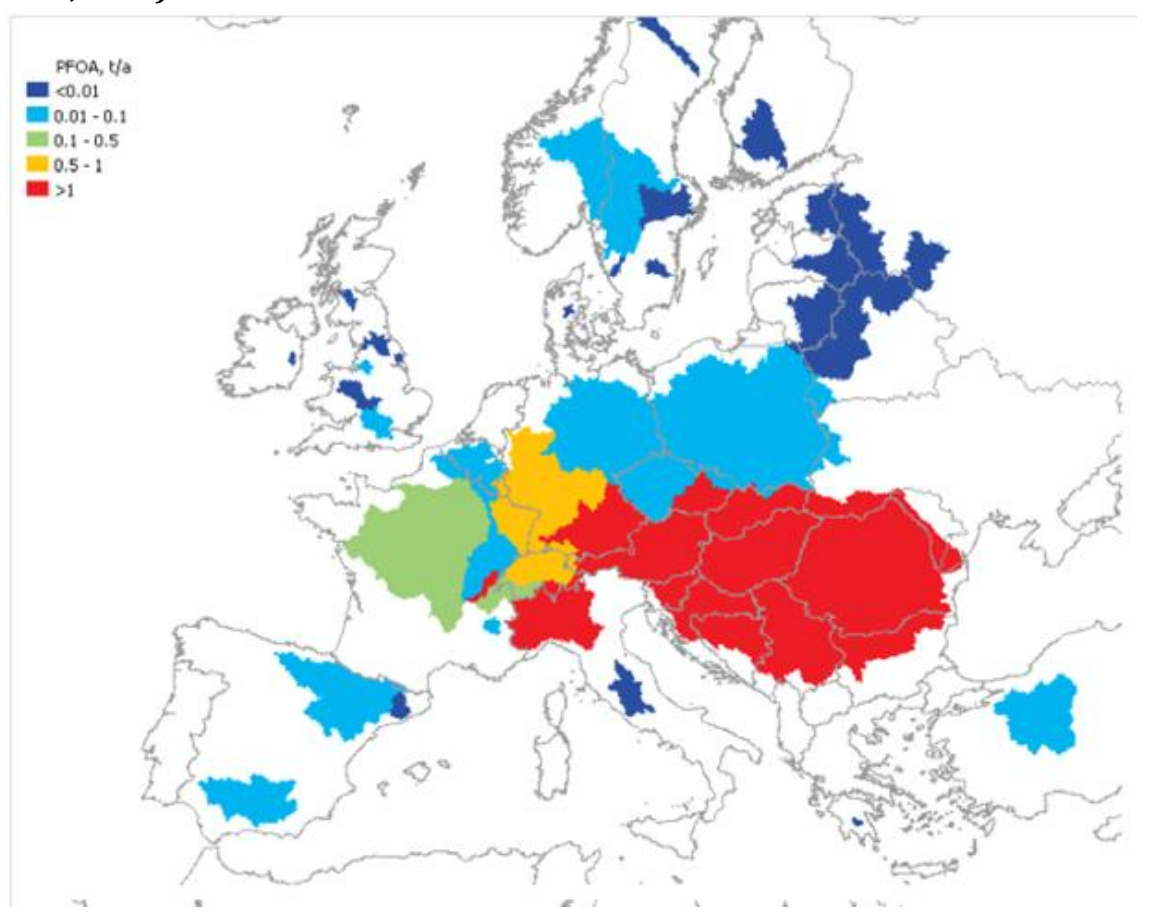


It can be seen that for the sites for which it was possible to estimate the emissions and concentrations, the Nordic countries were amongst the lowest found in all of Europe. This is also supported by the literature review of Eschauzier et al. (2008) who stated that "The low population density and fewer industrial activities in Scandinavian countries compared to central Europe could explain the lower concentrations found in the North of Europe."

Although it is difficult for someone to extrapolate and estimate exact annual emissions for entire countries, the data shows that the emission both in Norway and Sweden is much less than $100 \mathrm{Kg} / \mathrm{y}$ (Table 6).

The latter value is calculated by using the average European emission factor value Pistocchi and Loos (2009) estimated for PFOA (and also PFOS). The estimated emissions are given in Table 6; it should be noted that these emissions represent a relatively negative scenario and are overestimated. The reason for this overestimation is the fact that the average European emission factors are used, although, as it can be seen from figures 5 and 6, the emissions in Sweden and Norway are far lower than the average European emissions. For this purpose, it is safe to say that the emissions are below $100 \mathrm{Kg} / \mathrm{y}$ in each country, showing a declining trend for the Nordic emissions.

Table 6. Average emission factors and annual emission estimates for Norway (assuming population of 5 milions) and Sweden (assuming population of 9.5 milions)

\begin{tabular}{llll}
\hline & & $\begin{array}{l}\text { Emission Factors } \\
(\mu \mathrm{g} / \mathrm{d} \text { *capita) }\end{array}$ & Annual emissions $(\mathrm{Kg} / \mathrm{y})$ \\
\hline \multirow{2}{*}{ Norway } & PFOA & 27 & 49.28 \\
& PFOS & 19 & 34.68 \\
\multirow{3}{*}{ Sweden } & PFOA & 27 & 93.62 \\
& PFOS & 19 & 65.88 \\
\hline
\end{tabular}

Finally, the study of Pistocchi and Loos (2009) presented estimates for the surface water concentrations all over Europe, both for PFOA and PFOS (Figures 7 and 8).

It is interesting to note that the levels of PFOS and PFOA concentrations that were estimated (predicted) by Pistocchi and Loos (2009) are in very good agreement with the measured concentrations (few tens to few hundred $\mathrm{pg} / \mathrm{L}$ ). 
Figure 6. PFOS emission estimates for Europe (Figure taken from Pistocchi and Loos, 2009)

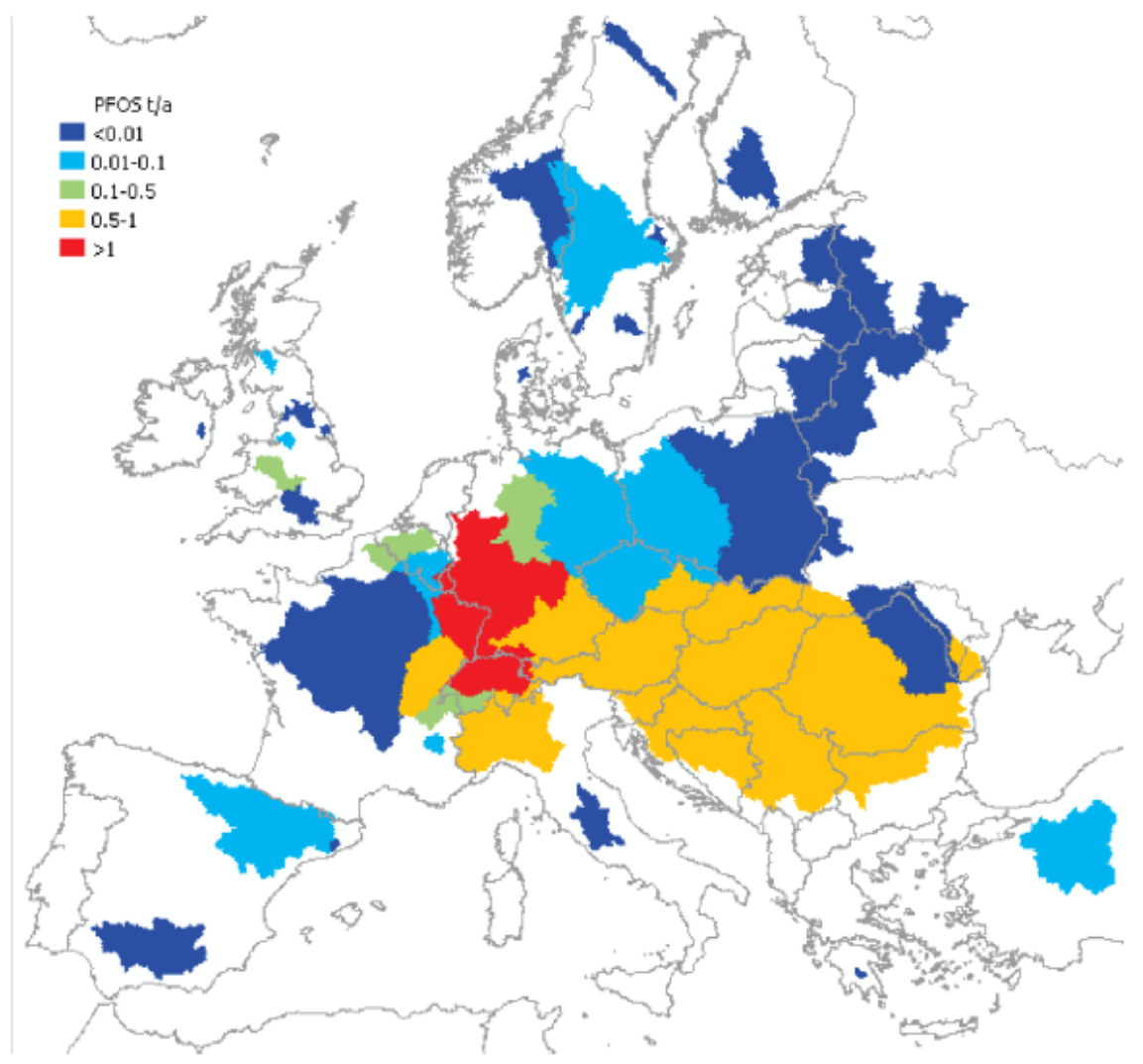


Figure 7. Surface water PFOA concentrations for Europe (Figure taken from Pistocchi and Loos, 2009)

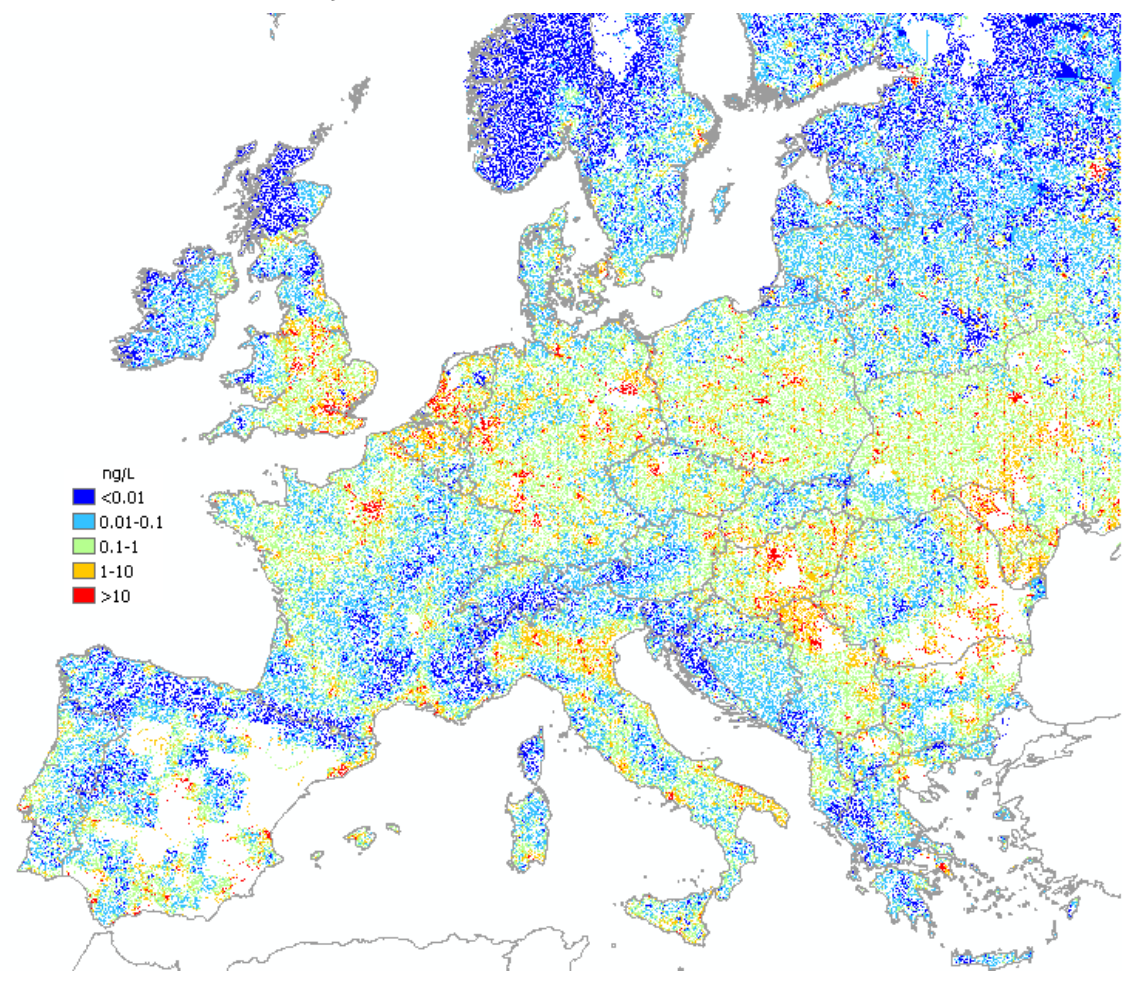


Figure 8. Surface water PFOS concentrations for Europe (Figure taken from Pistocchi and Loos, 2009)

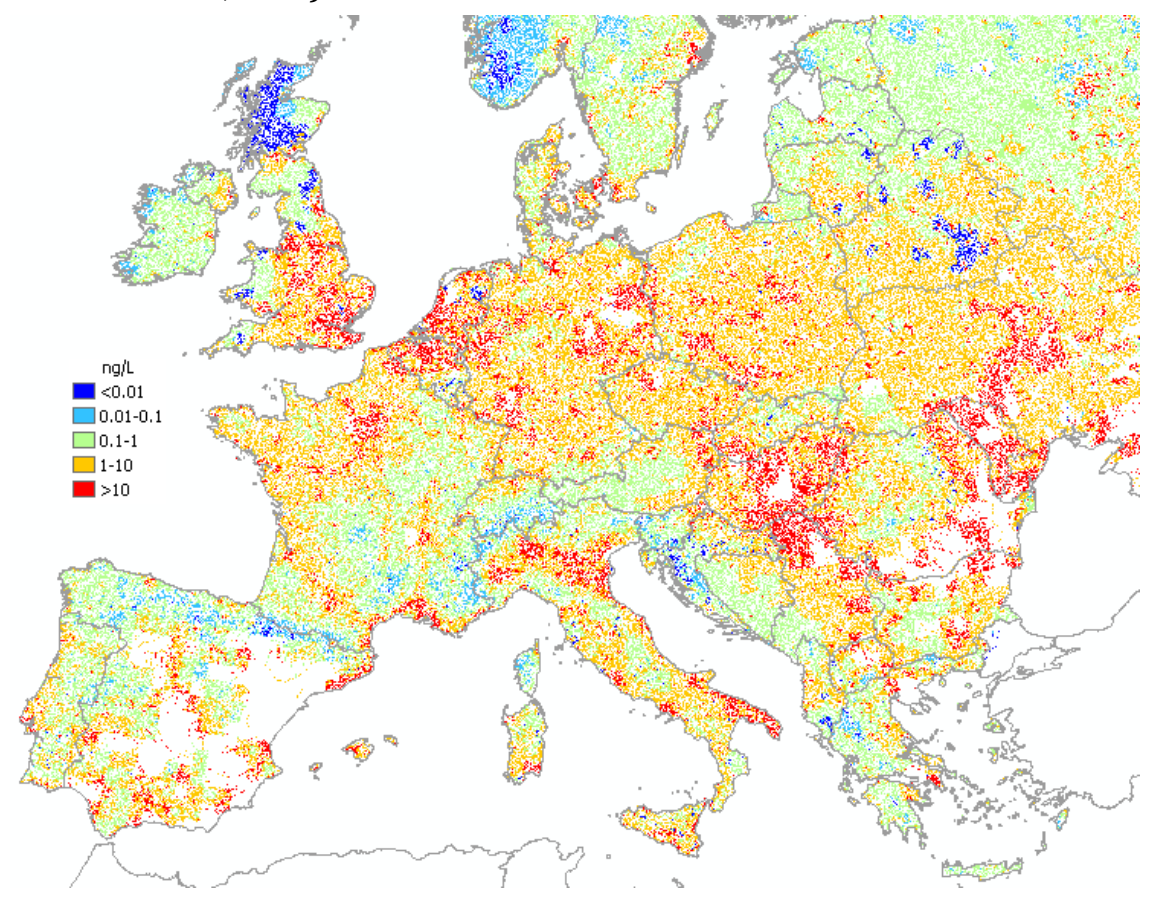

\subsubsection{Estimation of long range atmospheric transportation potential and fugacity modelling}

It is well known that the potential of individual organic contaminants to be transported over large distances (LRAT, long range atmospheric transport) will differ widely, reflecting differences in physico-chemical properties and reactivity in the atmosphere (Wania, 2006; Wania and Mackay, 1996). The $L_{A}$ (characteristic travel distance or long range atmospheric transport potential - LRATP) of any chemical at any point in time will be limited by atmospheric reaction and (net) atmospheric deposition. $\mathrm{L}_{\mathrm{A}}$ is an easy measure of the chemicals' mobility in the environment and is defined as the distance over which the initial air concentration of a chemical is reduced to 1/e ( 37\%) (Bennett et al., 1998; Beyer et al., 2000; Breivik et al., 2006). The LA can be estimated by the formula:

$\mathrm{L}_{\mathrm{A}}=\mathrm{u} \cdot \mathrm{M}_{\mathrm{A}} /\left[\mathrm{N}_{\mathrm{RA}}+\mathrm{N}_{\mathrm{AS}}\right]$

(Eq. 1), where $\mathrm{u}$ is the wind speed, and $\mathrm{N}_{\mathrm{RA}}$ and $\mathrm{N}_{\mathrm{AS}}$ are the rates or reaction and air to surface deposition, respectively.The calculation of $\mathrm{L}_{\mathrm{A}} \mathrm{S}$ for 
any substance allows us to estimate the concentrations at any distance from the source, by using the equation:

$C(x)=C_{0} \cdot e-x / L_{A}$

(Eq. 2) where, $\mathrm{C}(\mathrm{x})$ is the concentration of the chemical in a distance " $\mathrm{x}$ " from the emission point, $\mathrm{C}_{0}$ is the initial concentration of the chemical at the point of the emission (distance is $0 \mathrm{~km}$ ) and $\mathrm{L}_{\mathrm{A}}$ is the characteristic travel distance of the chemical.

This approach could be used to estimate LRATP for fluorinated compounds that are volatile and can be found in the gas phase. It would improve our understanding about the secondary emissions and about the contributions from non-Nordic countries. However, in order to do so, we need a rich set of monitoring data and sound and internally consistent physico-chemical properties (reaction rates, deposition rates) and in addition, values that are representative for the climatologic conditions in the Nordic countries. Both are important data gaps that should be better positioned/addressed within the future research priorities for the Nordic countries. Estimation of LRATP for all fluorinated chemicals is also beyond the scope of this report.

Another interesting tool for the estimation of the distribution of our target compounds between the various environmental compartments and thus, again, for the estimation of fluxes of fluorinated compounds that partition between air, water, soil, sediment etc, is the fugacity model (e.g. the LEVEL III model). This model requires as input the important environmental concentrations (air, water, soil etc.), emission rates and physico-chemical properties, in particular, the half-lives of the compounds of interest in the various environmental compartments. The LEVEL III model can then estimate directly loads, fluxes and concentrations, and can provide in a graphical form all the information about the "circle" of the pollutants between the various environmental compartments (Figure 9).

Application of the LEVEL III fugacity model is not possible for most fluorinated compounds of interest because of the lack of consistent and reliable data for the aforementioned parameters. Before such modelling approaches can be applied for the less studied fluorinated substances (e.g the long carbon chain ones, $>$ C8), we would need to have internally consistent physico-chemical parameters representative for the climatologic conditions of the Nordic countries. In addition, we would require emission factors and environmental concentrations in order to estimate 
accurately their environmental fate. At this point, for the less studied fluorinated compounds, this appears to be premature.

Figure 9. Graphical output of the LEVEL III model (the case of HBCDD)

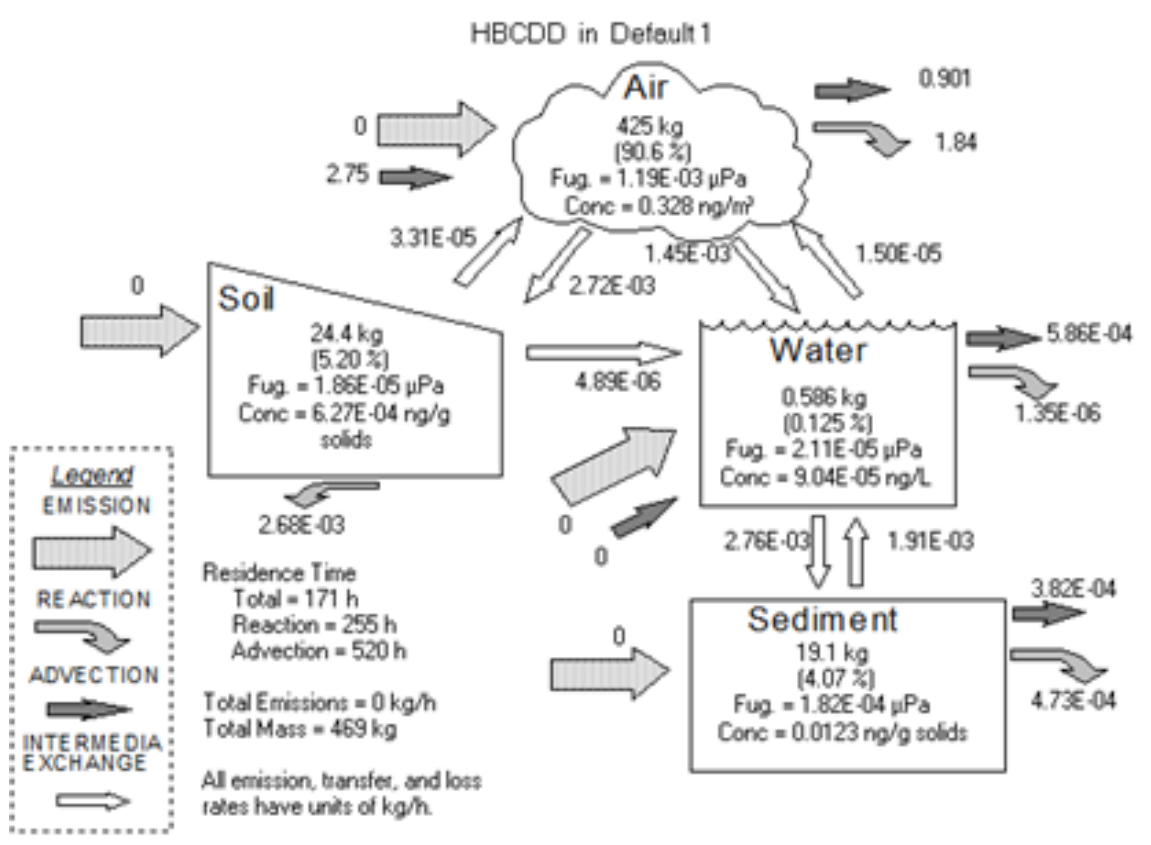

\subsubsection{Conclusion on emissions and occurrence}

PFCs in the environment in Nordic countries have been reported in publications and reports covering both biotic and abiotic samples, like air, indoor dust, water, wastewater, sludge, sediment and soil. Regarding PFCAs, most studies report finding PFOA, PFHXA and PFNA. Similarly, for PFSAs, PFOS and PFHxS are the most studied compounds. Compared to other countries, the concentrations in the Nordic countries are lower, especially when compared with central European countries with high GDPs. However these substances have also been found in the Arctic, far from any sources, which shows that these substances are global contaminants.

The outcome of this review of the environmental occurrence of fluorinated substances is that there is urgent need for new data, on more PFCs in order for decision makers to have a complete picture about the PFC levels in all environmental compartments and an in-depth knowledge of spatial and temporal distribution, and clear temporal trends. The detailed environmental fate study of PFCs is hindered in many cases by the lack of reliable (or in some cases total lack of) physical-chemical properties for many fluorinated compounds. 
Existing data on the emissions and surface water concentrations of PFOA and PFOS show that the Nordic countries are among the least contaminated regions in Europe from PFCs, as is to be expected due to lower population density and less industry.

\subsection{Sources of exposure of PFCs to humans}

In general there are two important sources of exposures of PFCs to humans namely via food and drink intake and through exposure to house dust.

Food intake is assumed to be a main source of exposure of the general population to PFCs. However most of the data are given on PFOS and PFOA whereas only limited data are available on other PFCs in food. In a recent study by Haug et al., 2010a, 12 different PFCs were detected in 21 samples of different food and beverages on the Norwegian marked (data in Appendix F). Calculation of intake was done by use of consumption data given by Norkost 1997 (Haug et al., 2010a). The study found that in general the highest dietary intake of PFCs in Norway was from PFOA (31 ng/day) followed by PFOS (18 ng/day), PFDA (13 ng/day) and PFNA (9.5 ng/day). For all the given substances $85 \%$ of the measured data was $>$ LOD. (A value of $1 / 2 \times$ LOD was used for data below LOD). The estimated total intake of the 12 PFCs for the Norwegian adult population was $103 \mathrm{ng} /$ day. Consumption of fish, meat, seafood products and cereals represented $75-92 \%$ of the total estimated intake of the PFCs.

In the UK the highest levels of 11 PFCs (PFHxA, PFHpA, PFOA, PFNA, PFDeA, PFUnA, PFDoA, PFBS, PFHxS, PFOS and PFOSA) were in fish and offal food (Clarke et al., 2010). Other kinds of food, including shellfish, meat, milk, butter, cheese, cereals and vegetables were found to be almost free of PFCs in the UK foodstuffs.

The intake from cereals is higher in the Nordic study (Haug et al., 2010a) than in a Spanish study (Ericson et al., 2008). This may be due to different consumption patterns in Norway and Spain or the Nowegian data on cereals may be overestimated due to analytical uncertainties, according to Haug et al. 2010a.

The estimated human intake of PFCs decreases with increasing age and the intake was found to be higher in males than in females according to Haug et al., 2010a. 


\subsubsection{PFCA (Perfluoro carboxylates)}

\section{Food and drinking water}

The median human intake of PFOA in several regions studied world wide is estimated to $2.9 \mathrm{ng} / \mathrm{kg}$ bw/day (Fromme et al., 2007) and 2.5 $\mathrm{ng} / \mathrm{kg} \mathrm{bw} /$ day (range 0.3-140 ng/kg bw/day) (Vestergren et al., 2008). Precursor compounds (as FTOH) used in the production of fluorinated polymers may add to the exposure of PFOA; this is especially the case in high-exposure scenarioes (sum of $95^{\text {th }}$ percentile values for each individual input values) (Vestergren et al., 2008) where precursor-based exposure to PFOA account for $48-55 \%$ of the total daily doses for adults.

The estimated intake of PFOA in the Norwegian population was found to be lower than what has been reported from Spain, Germany, UK, Canada and Japan (Haug et al., 2010b). Estimated dietary intakes of different PFCAs in the Norwegian population are given in the table below (table 7). The major PFC intake is from PFOA (31 ng/day) according to Haug et al., 2010a. The estimated intake of PFOA from the duplicate diet study given by Fromme et al., 2007 is 5-6 times higher than the intake of $31 \mathrm{ng}$ PFOA /day estimated by Haug et al, 2010a and $42 \mathrm{ng} /$ day (Haug et al., 2010b) (see table 7 on dietary intake below). This can be due to several parameters related to e.g. the differences in consumption pattern and the different levels of PFCs in food from the different countries as well as uncertainties in estimating the consumption of different foods. According to the Norwegian data, cereals give a major contribution to the intake of PFOA (see table 6) and in the total intake of PFCs cereals may also contribute significantly (Haug et al., 2009a and 2009b). In Norway PFOA in bread was estimated to be a major source of the total intake of PFCs (Haug et al. 2010b).

Fish is assumed to be a major source of fluorinated substances. This was also found in a Baltic study ( $n=45$, age 19-62) where individuals $(n=15)$ who declared to have a high fish consumption (mainly Baltic fish) on average showed the highest load (in blood samples) of the fluorinated substances of: PFHxA, PFHpA, PFNA, PFDA, PFUnDA, PFDoDA and to a lesser extent PFOA (Falandysz et al., 2006). In a Norwegian study fish and shellfish were estimated to give the largest contribution of PFOA and PFUnDA for human intake (38\% versus $93 \%$ ), calculated from correlation between serum PFC concentration and food consumption data. As can be seen from Table 1 in Appendix F, the levels of PFOA in fish found in the Norwegian study (Haug et al., 2010a) were significantly lower than the data from the UK (Clarke et al., 2009) and also lower compared to some other studies according to Haug et al., 2009. According to the author (Haug et al, 2009) this could be explained by the Nordic fish being caught in open sea rather than costal areas and due to different fish species 
Table 7. Dietary intake of perfluorocarboylates, PFCA, (ng/day) for the general Norwegian population

\begin{tabular}{|c|c|c|c|c|c|c|c|c|c|c|c|}
\hline Reference & Food type & Country & $\begin{array}{l}\text { Number of } \\
\text { samples* }\end{array}$ & Year & PFHXA & PFHpA & PFOA & PFNA & PFDA & PFUnDA & PFDoDA \\
\hline \multirow[t]{15}{*}{ Haug et al., 2010a } & Cereals & Norway & & 3 & 4.3 & 3.2 & 15.0 & 2.8 & 5.2 & 2.2 & 2.2 \\
\hline & Milk and dairy products & & & 3 & 1.3 & 2.0 & 4.4 & 4.4 & 2.7 & 1.4 & 2.2 \\
\hline & Fish and seafood & & & 3 & 0.55 & 0.91 & 2.4 & 0.44 & 1.2 & 1.0 & 0.36 \\
\hline & meat and meat product & & & 3 & 0.35 & 1.0 & 2.7 & 0.94 & 1.7 & 0.68 & 0.40 \\
\hline & Eggs & & & 3 & 0.22 & 0.13 & 0.49 & 0.06 & 0.21 & 0.17 & 0.07 \\
\hline & Sugar and sugar products & & & 3 & 0.06 & 0.04 & 0.25 & 0.07 & 0.16 & 0.12 & 0.12 \\
\hline & Fats & & & 3 & 0.08 & 0.09 & 0.40 & 0.22 & 0.14 & 0.27 & 0.27 \\
\hline & Vegetables & & & 3 & 0.11 & 0.06 & 0.25 & 0.10 & 0.10 & 0.13 & 0.17 \\
\hline & Starchy roots and potatoes & & & 3 & 0.39 & 0.14 & 0.66 & 0.26 & 0.38 & 0.28 & 0.30 \\
\hline & Fruits and juices & & & 3 & 0.12 & 0.09 & 0.36 & 0.08 & 0.14 & 0.11 & 0.14 \\
\hline & Coffee, tea and cocao & & & 3 & 0.18 & 0.25 & 2.1 & 0.07 & 0.29 & 0.12 & 0.22 \\
\hline & Alcoholic beverages & & & 3 & 0.04 & 0.04 & 0.15 & 0.01 & 0.06 & 0.02 & 0.02 \\
\hline & Tap water & & & 3 & 0.14 & 0.14 & 0.54 & 0.04 & 0.21 & 0.08 & 0.08 \\
\hline & Soft drinks & & & 3 & 0.12 & 0.12 & 0.45 & 0.03 & 0.18 & 0.06 & 0.07 \\
\hline & Total intake & & & & 8.0 & 8.2 & 31 & 9.5 & 13 & 6.7 & 6.7 \\
\hline \multicolumn{12}{|c|}{ Number of samples*: 3 different brands/types of each food were pooled } \\
\hline \multicolumn{12}{|c|}{ Note: Food consumption based on Norkost 1997 survey on 2672 adults } \\
\hline Haug et al., 2010b & Mean dietary intake of a 7 & $70 \mathrm{~kg}$ person & & & & & 42 & & & 24 & \\
\hline
\end{tabular}


The largest intake of PFOA may occur from contaminated food included drinking water (Trudel et al., 2008). According to Trudel et al., 2008 this is followed by the ingestion of dust and inhalation of air. The uptake of PFOA in children on a body weight basis is higher compared to adults because of a higher relative uptake from food and hand- mouth transfer from treated carpets and ingestion of dust (Trudel et al., 2008). In the high product scenarios the dominating pathways are found to be product- and age dependent: E.g. uptake from food contact materials is an important pathway for teenagers and adults (Trudel et al., 2008).

Drinking water may be a significant source of $\mathrm{PFC}$, and in particular PFOA, exposure to human. In drinking water, produced from surface water in contaminated areas, PFOA was the main compound found in a German study with the level of 500-640 ng/L (Hölzer et al., 2008). This is in accordance with another German study reporting high levels of PFOA (519 ng/L) followed by PFHpA (23 ng/L) and PFHxA (22 ng/l) (Table 2 in Appendix F) in public water supplies produced from river water with bank filtration or artificial recharge (Skutlarek et al., 2006). When activated-charcoal filters were installed in the water supply, this efficiently decreased the PFC concentration in drinking water (Skutlarek et al., 2006). In other areas the level of PFCs in drinking water was much lower, with the sum of PFCs varying between non-detected and $27 \mathrm{ng} / \mathrm{L}$ (Skutlarek et al., 2006). In the Netherlands the level of PFCs in drinking water resources was found to be in the range of non-detectable to $43 \mathrm{ng} / \mathrm{l}$ (Mons et al., 2007). In a recent study by Haug et al., 2010a, three samples of tap water from different Norwegian water works in the Oslo area were analysed. The level of PFOA was $0.65-2.5 \mathrm{ng} / \mathrm{L}$ whereas the other PFCAs were below $1 \mathrm{ng} / \mathrm{L}$ as given in Table 2 in Appendix F. A review on the presence of PFCAs and PFSAs in European surface waters, ground water and drinking water was recently published as a book chapter (Eschauzier et al. 2012). It compared the relative importance of different sources of intake of PFCs, and showed that where raw water was affected by point contamination, e.g. by contaminated sludge, then the corresponding drinking water was the major source of human exposure. This is also shown for the intake of PFOA in the figure below (pie c) compared to different other exposure scenarios (pie a, b and d) (Vestergren et al., 2009). 
Figure 10. Pie charts displaying a compilation of the estimated daily intakes of $P F O A$ for male adults (D) and relative importance of exposure pathways from separate studies
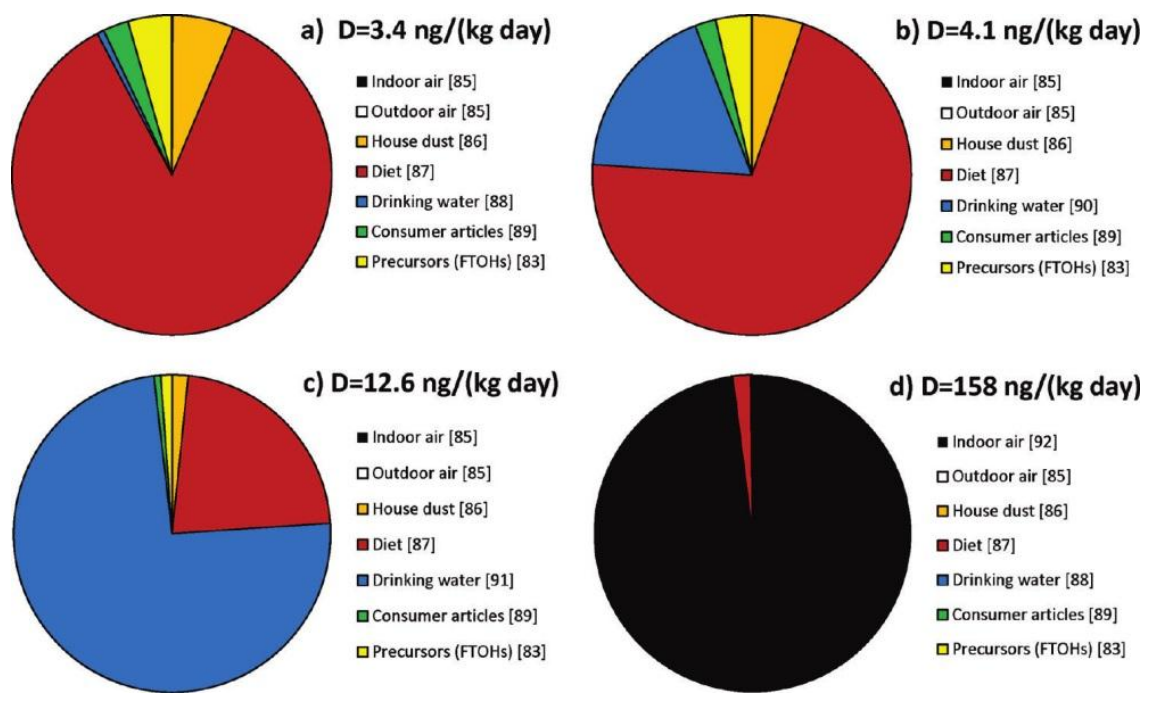

Each pie chart represents an exposure scenario representative of (a) background concentrations in drinking water (1.3 ng/L); (b) elevated concentrations in drinking water (40 ng/L); (c) point sources of drinking water contamination (519 ng/L); (d) occupationally exposed individuals (indoor air concentrations $1 \mu \mathrm{g} / \mathrm{m} 3$ ). References of the individual studies are given in square brackets in the legends of each chart. (Vestergren et al., 2009)

In a recent study, tap water from six European cities were analysed for PFCAs. The higest level of PFCA was found for PFOA ( $8.6 \mathrm{ng} / \mathrm{l})$ in water samples from Amsterdam (Ullah et al., 2011).

\section{Food packaging materials}

In a recent Danish study 84 different samples of food pakaging materials of paper and board were tested for contents of per-and polyfluorinate compounds by exposure to $50 \%$ ethanol. In 35 of the samples the level of PFCs were above the the limit of detection. High levels of PFCA were found in the extracts of popcorn bags (Trier et al., 2012).

\section{Consumer products and cosmetics}

PFCs are primarily used as processing aids in the manufacture of fluoropolymers and can be detected either as additives or residual impurities (with a content from C4 to C14) in a large variety of commercial products, including leather, carpets, paper, paint, AFFF, waterproofing agents, coated fabrics, non-stick cook ware, floor wax (dominant contributions from PFHpA, PFOA, and PFNA), ski wax and textiles and clothes (Begley et al., 2005; Freberg et al., 2010; Gewurtz et al., 2009; 
Prevedouros et al., 2006; Sinclair et al., 2007; Trudel et al., 2008; Washburn et al., 2005). Herzke et al. (2012) found that none of the waterproofing agents/lubricants they analysed were free from PFCs, most abundant being PFBS, PFBA, PFNA, PFDoA, PFHxA and PFHpA (see Table 1 Appendix E). They also detected PFCAs in table cloths, presumably due to Teflon treatment, but they could not establish if the minimal levels found in paint were actually added or only result of contamination. PTFE or Teflon ${ }^{\circledR}$ is probably the most publicly well-known and most widely used fluoropolymer as a source of PFCAs (Walters and Santillo, 2006). Applications of PTFE include: electrical wire insulation, tape, waterproof membranes for garments (such as Gore-Tex), surgical implants, dental floss, engine protector additives, non-stick coatings, moulded parts and coatings for use in a wide range of chemically hostile environments (DuPont, 2012).

Consumer products like sprays and treated carpets may contribute to the consumer exposure of PFOA (Trudel et al., 2008) but are probably a less important source for most consumers/the general population according to Trudel et al., 2006. However, these sources may contribute significantly to the exposure for those consumers frequently using e.g. PFC containing sprays and who have treated carpets in their home (Trudel et al., 2008). Table 1 in Appendix E gives an overview of the presence of PFCs in consumer products.

\section{Indoor air exposure}

All PFCAs have been detected in indoor house dust in Norwegian houses and offices (Huber et al., 2011). In the latter study, PFUnA was the most abundant PFCA in houses with a median concentration in dust of 120 $\mathrm{ng} / \mathrm{g}$, followed by PFOA (38.8 ng/g) and PFHxA (10.1 ng/g). In one office reported in the same study, the pattern was different, with PFOA being the most abundant chemical (694 ng/g), followed by PFHxA (29.3 ng/g), while PFUnDA was among the least abundant, exhibiting the concentration $1.4 \mathrm{ng} / \mathrm{g}$. Haug et al. (2011) also reported concentrations of PFCAs in indoor dust from Norwegian homes. In this particular study, PFHxA was the most abundant chemical in dust (33 ng/g), followed by PFNA (29 $\mathrm{ng} / \mathrm{g}$ ) and PFOA (20 ng/g). This was the only study that reported also detectable concentrations of PFTrDA and PFTeDA in indoor dust, suggesting that indoor air dust can be a sink for many compounds that occur in low levels in the indoor air. Finally, in a study from Sweden (Bjorklund et al., 2009), PFOA was studied in houses, offices and apartments and the average concentrations were 54, 93 and $70 \mathrm{ng} / \mathrm{g}$, respectively, thus, in the same order of magnitude as in Norwegian indoor environments. 
PFHxA (17.1 pg/m3), PFOA (4.4 pg/m³) and PFNA (2.7 pg/m³) were detected also in indoor air particles in Tromsø (Barber et al., 2007).

To the best of our knowledge, the only study that has quantified exposure of humans to PFOA in indoor air in the Nordic countries is the study of Haug et al. (2011b). In the latter study, it was shown that through indoor air dust, the uptake of PFOA through dust will range between 0.19 and $0.78 \mathrm{ng} / \mathrm{kg}$ bw/day and through air the same uptake will be between 0.002 and $0.16 \mathrm{ng} / \mathrm{kg}$ bw/day. It was shown that uptake through dust and air was particularly low compared to other exposure pathways.

\subsubsection{PFSA (Perfluoroalkyl sulfonates)}

\section{Food and drinking water}

Human intake of PFOS has been estimated to a wide range of 3.9$530 \mathrm{ng} / \mathrm{kg}$ bw/day (Vestergren et al., 2007). Precursor compounds (as PFOSA and PFOSE) used in the production of fluorinated polymers may add to the exposure due to their degradation into PFOS. The median intake of PFOS was found to be $1.4 \mathrm{ng} / \mathrm{kg} \mathrm{bw} /$ day based on analysis of duplicate diet samples from various regions world wide $(\mathrm{n}=214)$ of 31 healthy individuals (age 16-45) (Fromme et al., 2007). PFHxS and PFHxA could only be detected in some samples (above the LOD) with a median intake of $2.0 \mathrm{ng} / \mathrm{kg} \mathrm{bw} /$ day and $4.3 \mathrm{ng} / \mathrm{kg} \mathrm{bw} /$ day. The estimated intake of PFOS from the duplicate diet study given by Fromme et al., 2007 is about 5 times higher than the intake estimated by Haug et al, 2010. This can be due to several parameters related e.g. to differences in consumption pattern and the level of the PFCs in food from the different countries, to uncertainties in estimating the consumption of different foods and to uncertainties regarding the analytical test methods and analysis.

As for PFOA, the largest intake of PFOS seems to occur from contaminated food included drinking water (Trudel et al., 2008). This is followed by the ingestion of dust and inhalation of air. Consumer products like sprays, treated carpets and food contact materials may also lead to consumer exposure of PFOS (Trudel et al., 2008) but as for PFOA the spray sources are probably less important for most consumers/the general population according to Trudel et al., 2008. However, spray sources may contribute significantly to the exposure for those consumers frequently using e.g. PFC containing sprays and who have treated carpets in their home (Trudel et al., 2008).

A recent Norwegian study found that in general the major dietary intake of PFCs in Norway was PFOS (18 ng/day) (and from PFOA 
(31 ng/day as given above)) (Haug et al., 2010a). The estimated intake of PFOS in this Norwegian study was found to be lower than what has been reported from Spain, Germany, UK, Canada and Japan (Haug et al., 2010b) and also lower than reported in another recent study of the same author (Haug et al., 2010b) as given in the table below. Different data on food consumption were used in the two Norwegian studies and may be one reason for the observed differences in the Norwegian PFOS intake.

In relation to age, the highest potential intakes of PFOS are estimated for infants and toddlers (Vestergren et al., 2007). The uptake of PFOS in children on a body weight basis tend to be higher because of a higher relative uptake from food and hand- mouth transfer from treated carpets and ingestion of dust (Trudel et al., 2008). In the high product scenarios the dominating pathways are found to be product- and age dependent: E.g. uptake from food contact materials is an important pathway for teenagers and adults (Trudel et al., 2008).

Table 8. Dietary intake of perfluoroalkyl sulfonates, PFSA (ng/day) for the general Norwegian population

\begin{tabular}{|c|c|c|c|c|c|c|}
\hline Reference & Food type & Country & $\begin{array}{l}\text { Number of } \\
\text { samples* }\end{array}$ & PFBS & PFHXS & PFOS \\
\hline \multirow[t]{15}{*}{ Haug et al., 2010a } & Cereals & Norway & 3 & 0.22 & 0.52 & 5.1 \\
\hline & Milk and dairy products & & 3 & 0.22 & 0.10 & 4.7 \\
\hline & Fish and seafood & & 3 & 0.19 & 0.19 & 3.4 \\
\hline & meat and meat product & & 3 & 0.14 & 0.09 & 3.3 \\
\hline & Eggs & & 3 & 0.03 & 0.06 & 0.66 \\
\hline & Sugar and sugar products & & 3 & 0.01 & 0.005 & 0.05 \\
\hline & Fats & & 3 & 0.03 & 0.04 & 0.08 \\
\hline & Vegetables & & 3 & 0.01 & 0.006 & 0.06 \\
\hline & Starchy roots and potatoes & & 3 & 0.03 & 0.01 & 0.13 \\
\hline & Fruits and juices & & 3 & 0.01 & 0.02 & 0.06 \\
\hline & Coffee, tea and cocao & & 3 & 0.01 & 0.05 & 0.10 \\
\hline & Alcoholic beverages & & 3 & 0.002 & 0.01 & 0.02 \\
\hline & Tap water & & 3 & 0.008 & 0.04 & 0.08 \\
\hline & Soft drinks & & 3 & 0.007 & 0.03 & 0.06 \\
\hline & Total intake & & & 0.93 & 1.2 & 18 \\
\hline \multicolumn{7}{|c|}{ Number of samples*: 3 different brands/types of each food were pooled } \\
\hline \multicolumn{7}{|c|}{ Note: Food consumption based on Norkost 1997 survey on 2672 adults } \\
\hline & & & & & & \\
\hline Haug et al., 2010b & Mean dietary intake of a 70 & & & & & 105 \\
\hline Note: Food consur & mption based on frequency & ares & & & & \\
\hline
\end{tabular}

Fish and shellfish were estimated to contribute with $81 \%$ of the total PFOS intake (Haug et al., 2010b). In general the level of PFOS in fish is found to be higher than the level of PFOA (Fromme et al., 2009). This is in accordance with a recent minor Danish study on PFOS and PFOA in fish from Danish waters where the average level of PFOS was found to be $1,8 \mathrm{ng} / \mathrm{kg}$ ( $n=9$ ) (Granby, 2012, unpublished data) whereas the level of PFOA was < 0.5 (LOD). In a German study PFOS was detected in 33 wild fish $(\mathrm{n}=112)$ at a concentration up to $225 \mathrm{ug} / \mathrm{kg}$ PFOS (Schuetze et al., 2010). 
PFOS was the PFC (of 11 PFCs analysed) most often detected in especially fish, shellfish, liver and kidney and most often at the highest concentrations in a UK study of 252 food samples (Clarke et al., 2009). In $70 \%$ of the samples none of the 11 analytes were present above LOD. The highest levels were $59 \mathrm{ug} / \mathrm{kg}$ PFOS and $63 \mathrm{ug} / \mathrm{kg}$ total PFCs in an eel sample followed by $40 \mathrm{ug} / \mathrm{kg}$ PFOS and $62 \mathrm{ug} / \mathrm{kg}$ total PFCs in a whitebait sample (Clarke et al, 2009).

Intake of fruit and vegetables seems to affect the level of PFOS and PFHpS. In a population of northern Norway the intake of PFOS and PFHpS was found to decrease significantly with the increased intake of fruit and vegetables (Rylander et al., 2009). The conclusion was based on food frequency questionnaire information from 60 adults (44 women and 16 men) in correlation to PFC levels in blood samples. Similary a study of a Danish birth cohort $(n=1,076)$ found a decrease in PFOS and PFOA concentrations with increased intake of fruit and vegetable (Halldorsson et al., 2008). In the latter study the correlation could be partly explained by a lower intake of red meat, animal fat and snacks. The authors discuss the possibility that the observed correlation between fruit/vegetables and blood PFC levels may be explained by a large number of confounding variables that characterize a healthy lifestyle (Halldorsson et al., 2008). It is recommended to include lifestyle factors and dietary patterns instead of single food groups in future studies (Rylander et al., 2009).

In tap water samples $(n=3)$ from the Oslo area the level of PFOS was 0.071-0.23 ng/L and the concentrations of PFBS and PFHS were below this (Haug et al. 2010a) as can be seen from Table 3 in Appendix F. In another recent study of PFCs in tap water from six European cities the higest levels of PFSAs were found for PFBS (18.8 ng/L) in tap water from Amsterdam and PFOS $(8.8 \mathrm{ng} / \mathrm{L})$ in tap water samples from Stockholm (Ullah et al., 2011).

\section{Food packaging materials}

Food contact materials may add to the human exposure of PFCs. In a recent Danish study 84 different samples of food pakaging materials of paper and board were tested for per- and polyfluorinate compunds, including PFOS and PFHxS. PFOS was not detected in any of the samples and PFHxS was only found in one sample of popcorn bag at a low level (Trier et al., 2012). 


\section{Consumer products}

Since PFOS was banned in most industrialised countries, the appearance of alternative perfluoroalkyl sulfonates has become more obvious. According to available data these compounds appear to have a main application in fire fighting foams and carpet protection products (Huber et al., 2011). However, Herzke et al. (2012) reported the detection of PFSAs (analysed were PFOSA, PFBS, PFPS, PFHxS, PFHpS and PFDcS) in several consumer products of different brands. These included, black shoe leather, office furniture leather carpet, paint, non-stick ware, waterproofing agents and coated fabrics. Novec ${ }^{\mathrm{TM}}$ from $3 \mathrm{M}$ is a fluorosurfactant containing PFBS and is an ingredient in different paints and coatings (3M 2012).

\section{Indoor air exposure}

Exposure to PFSAs in the indoor environment occurs mainly through dust. In the Nordic countries, PFSAs have been reported for Norwegian homes and in an office and similarly for Sweden, again for residences and offices. PFOS is the dominant PFSA with concentrations in dust that have reached $147.7 \mathrm{ng} / \mathrm{g}$ in a Norwegian office (Huber et al., 2011). Very high concentrations of PFOS have been detected also in the Swedish offices analysed by Bjorklund et al. (2009). In homes/residences, PFOS ranged between 9.1 and 11 ng/g in Norway and between 39 and 85 in Sweden. The lower levels in residences demonstrate the higher relative importance of occupational exposure compared to exposure in private homes. Among other PFSAs, PFHxS exhibited a concentration of $27.8 \mathrm{ng} / \mathrm{g}$ in the Norwegian office (Huber et al., 2011), being much higher than in homes (1.4-8.4 ng/g). In indoor air particles from Tromsø, only PFDS was detected $(2.6 \mathrm{pg} / \mathrm{m} 3)$.

The uptake rate has been calculated for PFOS (Haug et al., 2011b) and based on three different scenarios, this ranged for Norwegians between 0.11 and $0.46 \mathrm{ng} / \mathrm{kg} \mathrm{bw} /$ day, through dust, and between 0.004 and $0.36 \mathrm{ng} / \mathrm{kg} \mathrm{bw} /$ day, through air.

\subsubsection{PFAL (Perfluoro aldehydes)}

\section{Food and drinking water}

No exposure data were found for exposure to humans.

\section{Food packaging materials}

No exposure data were found for exposure to humans.

\section{Consumer products}

No information on PFAL in consumer products. 


\subsubsection{FTOH (fluorotelomer alcohols)}

\section{Food and drinking water}

Data missing.

\section{Food packaging materials}

In a recent Danish study 84 different samples of food pakaging materials of paper and board were tested for per- and polyfluorinate compounds by exposure into 50\% ethanol. PFCs were found in 35 of the samples. Fluorotelomer alcohols were found in high levels in different types of packaging materials as coffee bags, popcorn bags, and paper and board for take away food and cakes (Trier et al., 2012).

\section{Consumer products}

A variety of fluorotelomers, including FTOHs, are used in a wide range of commercial products and in some applications, such as fire fighting foams, as well as soil, stain, and grease-resistant coatings on carpets, textiles, paper, and leather, the FTOHs are directly released into the environment (Lehmler, 2005). The manufacture of FTOHs usually results in a mixture containing six to twelve fluorinated carbon congeners and are found in materials such as (see Table 1, Appendix E) Polyfox $®$, Teflon $\AA$ Advance carpet protector, Zonyl $囚$, Motomaster $\AA$ windshield washer and 8:2 Methacrylate (Dinglasan-Panlilio and Mabury, 2006; Herzke et al., 2012). Fluorotelomers are also found in Teflon $®$ frying pans, microwave popcorn packing paper, waterproofing agents and Forafac® 1,157 fire fighting foam (Herzke et al., 2012; Moe et al., 2012; Sinclair et al., 2007). In addition, FTOHs are manufactured as a raw material for use in the synthesis of fluorotelomer-based surfactants and polymeric products (Dinglasan-Panlilio and Mabury, 2006).

\section{Indoor air exposure}

Exposure to FTOHs can be an important exposure path, because of the volatile nature of FTOHs. It has been shown in indoor exposure studies (1Report 2367/2008; ${ }^{2}$ Haug et al., 2011; ${ }^{3}$ Huber et al., 2011; ${ }^{4}$ Barber et al., 2007; 5Jahnke et al., 2007.), that FTOHs in indoor air can reach very high levels and be tens or hundreds of times higher than in the outdoor air (Table 4). Due to the fact that FTOHs have been used in many household products, the primary emissions are expected to take place directly from the indoor environment. To the best of our knowledge, there is no study estimating the uptake of FTOHs due to indoor air occupancies. 


\subsubsection{FTS (fluorotelomer sulfonates)}

\section{Food and drinking water}

Data missing.

\section{Food packaging materials}

Data missing.

\section{Consumer products}

The FTSs are used among other fluorotelomers in fire fighting foam for their film forming properties and the ability to decrease fuel absorption. The quantities of FTSs in the foam are low, but the foam is released directly into the environment (Hagenaars et al., 2011a; Moe et al., 2012). Although most analysis for FTSs in soil samples are taken in close proximity to airports and airport fire training facilities (Hagenaars et al., 2011a; Moe et al., 2012), Huber et al. (2011) reported for the first time detection of FTSs in in-house dust samples.

\subsubsection{PAP/di-PAP (polyfluoroalkyl phosphate esters)}

\section{Food and drinking water}

Data missing.

\section{Food packaging materials}

Paper and board $(n=14)$ intended for food contact at high temperature were sampled from Danish retailers in 2008. Di-PAPs, tri-PAPs and SdiPAPs were detected in five of 14 samples (Xenia Trier et al., 2011).

In a recent Danish study 84 different samples of food pakaging materials of paper and board were analyzed for per- and polyfluorinate compounds, including mono- and di-PAPs (Trier et al., 2012). Mono-PAPs were only detected in a few samples and at low levels. Di-PAPs were found in several of the food contact materials tested. The highest level was found in a paper bag for flour containing several different di-PAPs (Trier et al., 2012). 


\subsubsection{Other fluorinated telomers}

\section{Food and drinking water}

Data missing.

\section{Food packaging materials}

Data missing.

\section{Consumer products (Cosmetic, Textiles)}

No data were found

\subsubsection{Other fluorinated compounds of interest}

\section{Food and drinking water}

Perfluoroctane sulfonamides were tested in Canadian food (Tittlemier et al., 2006). The most frequently detected substance was N-EtPFOSA that was found in 78 of the 151 samples followed by N-MePFOSA in 25 of 51 samples. The highest levels and frequency of detection of analytes were found in fast food composites as particularly in french fries $(9.7 \mathrm{ng} / \mathrm{g})$, egg breakfast sandwiches and pizza (27.3 ng/g) (Tittlemier et al., 2006).

\section{Food packaging materials}

In recent years there has been a shift away from fluorotelomer surfactants towards per- and polyfluorinated polymers, such as per- and polyfluorinated polyethers (PFPEs). On the European market there is currently a shift away from telomeric PFCs to PFPEs as coatings for popcorn bags and on fastfood packaging (e.g. McDonalds) (personal communication with a czech popcorn producer and supplier for $25 \%$ or the Nordic market for microwave popcorn bags, 2012). Solvay Solexis is a major producer of PFPEs. In samples taken from Denmark, Sweden and Canada in 2009, PFPEs were found in 7 (18\%) of 50 samples by measurement by 19F NMR (Trier, 2011, thesis).

\section{Consumer products}

PFPE (Perfluoropolyether, also called PFAE or PFPAE) is a clear, colourless fluorinated synthetic oil that is non-reactive, non-flammable, and long lasting. PFPE is used in greases, oils and lubricants and can be found with the trade names Fomblin ${ }^{\circledR}$ (Solvay plastics) in cosmetics, Molykote ${ }^{\circledR}$ (Dow Corning) in industrial grease, Krytox® (DuPont) in lubricants, Fluorolink $®$ and Galden $®$ (Solvay plastics) for miscellaneous use. Perfluorocarbon emulsions are used as artificial blood or blood substitutes (Goorha et al., 2003; Riess, 2002; Riess and Krafft, 1998). The 
first commercially available PFC blood substitute was Fluosol ${ }^{\circledR}$ and Oxypherol ${ }^{\circledR}$ from Castro IC and comprised two PFCs, perfluorodecalin (PFD) and perfluorotrypropylamine (PFTPA). PFD is oxygenated using a bubble-through technique with $100 \%$ oxygen and infused as a red blood cell substitute (Hoang et al., 2009). Chosen for the second generation PFC blood substitutes were PFD, perfluorooctylbromide (PFOB) and bis(perfluorobutyl)ethylene. PFOB is known in its emulsion as Oxygent (Alliance Pharmaceutical Corp.) and is found in the products Columbian emulsion ${ }^{\circledR}$ and French emulsion ${ }^{\circledR}$ from Castor IC.

\subsubsection{Conclusion on food and drinking water and consumer products}

\section{Food and drinking water}

In the last years several papers have been published on PFCAs and PFSAs in food. Based on these data fish is assumed to be a major source of human exposure of PFCs from food. The levels of of PFOS and PFOA in fish from Norway were found to be significantly lower than the levels found in several other studies. According to Norwegian data, cereals (including bread) seem to be another major source to the intake of PFOA and to the total intake of PFCs.

When estimating the human intake of PFCs the intake of e.g. PFOA was found to be significantly lower in the Norwegian population than what has been reported from Spain, Germany, UK, Canada and Japan. This can be due to several parameters related to e.g. differences in consumption pattern and different levels of PFCs in food from different countries as well as uncertainties in estimating the consumption of different foods. Of course analytical uncertainties concerning PFCs have to be considered as well.

The level of PFCs in drinking water can vary a lot, and it may be a significant source of PFCs if the drinking water is produced from surface water in contaminated areas and where drinking water is affected by point contamination, as e.g. by contaminated sludge. In tap water from Stockholm and Oslo PFOS and PFOA were found at lower levels and for several other PFCs the levels are below the LODs. Drinking water seems not to be a major source of PFCs in these countries.

Only very few data are published on non-PFCA and non-PFSA in food. One reason for this is the analytical challenge in analysing these substances and therefore adequate and good performance analytical methods are a great need in this field. Several different PFCs have been found in food contact materials, including PFCA, PFSA, FTOH and PAPs. Food contact 
materials may be a significant source of PFC contamination of food. At the time being more data on migration from food contact materials into food of PFCs and especially of non PFCA and non PFSA are needed, to estimate the human exposure of PFCs from food contact materials.

\section{Consumer products}

The presence of PFCs in a broad range of consumer products can give rise to a constant diffuse human exposure in the developed parts of the world. Consumer products may therefore be a significant source of PFC exposure to humans, although, estimating exposure via consumer products includes large uncertainties, e.g. brand, volume and number of usage frequency differs between individuals. In addition, the overall human exposure due to PFC treated products might be low in general, but particular sub-groups in the population may receive considerably higher doses than the rest. Direct skin exposure from a skin care product, inhalation of aerosols from an impregnation spray or the use of a blood substitute product may occasionally be important routes of exposure, but are difficult to quantify. Further, information on chemical content of different consumer products is often severely limited, especially on nonPFOS/PFOA PFCs, since the composition of technical applications and mixtures of active ingredients are mostly confidential. Consequently, there is scant knowledge of PFAS content in consumer products and as a consequence we know little about possible emissions of PFAS from consumer products (Dinglasan-Panlilio and Mabury, 2006; Fiedler et al., 2010; Herzke et al., 2012; Sinclair et al., 2007). Literature search gives a certain overview of consumer products both for industrial and personal use available on the market as shown in Tables 1 and 2 in Appendix D. Literature searched included analytical publications where consumer products were analysed and certain PFCs were screened for, as well as the available producer information accessible online. Patents were not included in this overview as they do not necessarily indicate a usage, rather than the compound merely existing. The main products include non-stick cooking ware, coated textiles, footwear, food packaging material, cosmetics, repellent and impregnation products, etc. Final assessment of content of non-PFOS/PFOA PFCs in consumer products indicates a large gap of knowledge. 


\subsection{Occurrence of PFCs in humans}

PFCs are ambiphilic and bind to serum proteins and proteins in cell membranes, and accumulate in blood and internal organ such as liver, kidneys, testes and brain (Jones et al., 2003).

Generally the elimination half-life ${ }^{23}$ of PFCs in humans is enhanced with decreasing carbon-chain length: PFHxS (8.5 years), PFOS (5.4 years), PFOA (2.3-3.8 years), PFBS (1 month) and PFBA (3 days) (reviewed by (Lau, 2012)).

Most peer-reviewed literature contains reports on perfluorinated alkyl substances (PFAAs) ${ }^{24}$ detected in blood (whole blood, plasma and serum) across the world. Blood levels of perfluorinated chemicals have been monitored in many countries and usually PFOS, PFOA, and PFHxS are detected most frequently, and PFOS is detected at the highest concentrations, followed by PFOA and PFHxS. Other PFCs detected in human tissue include PFOSA, Me-PFOSA-AcOH, Et-PFOSA-AcOH or PFOSAA, PFNA, PFDA, PFUA, PFDoA, PFPeA, PFHxA, and PFBS. The short-chain perfluorinated acids are typically not monitored in human sera analysis, but in the case of detection, the concentrations are usually below or close to the limit of quantification (LOQ).

In the following we present the monitoring data found for the Nordic countries (see Tables 9, 1011 and 12).

\section{Levels in blood}

In most studies blood serum is the material analyzed but some studies analyze whole blood or blood plasma. PFC levels in serum and plasma are comparable (1:1) regarding PFOS, PFOA and PFHxS, but levels in whole blood are 2-3 times lower than serum (Ehresman et al., 2007).

\footnotetext{
${ }^{23}$ Half-life is a characteristic parameter of a substance's persistence. If a substance has a half-life greater than two months in water or six month in soil or sediment it is considered as persistent (Annex D, Stockholm Convention).

${ }^{24}$ See appendix A.
} 


\subsubsection{PFCA (Perfluorocarboxylic acids) in humans}

In the following the monitoring data for PFCA are described for each Nordic country (see Table 9). No biomonitoring data were found for Iceland and Finland.

\section{Norway (NO)}

The concentration and time trends of 19 different PFCs for the general Norwegian population were investigated in a cross sectional study by Haug and coworkers (Haug et al., 2009). Archived sera from men of age 40-50 years sampled from different county hospitals in Norway during 1976-2007 were pooled $(\mathrm{n} \geq 20)$ and analyzed.

The concentration of PFOA was $2.7 \mathrm{ng} / \mathrm{ml}$ in 2006. In most samples, PFNA, PFDA, PFUnDA and PFTrDA (median range: PFNA 0.55 to PFTrDA $0.06 \mathrm{ng} / \mathrm{ml}$ ) were detected, while PFPeA, PFHpA and PFDoDA were found less frequently. PFBA, PFHxA, PFTeDA were not observed above LOQ in any of the samples.

Trends: The pooled serum samples showed an increase of PFOA (9fold), PFNA, PFDA, and PFUnDA from 1976 to the mid-1990s where the concentrations stabilized. During 2000 to 2006, the PFOA level decreased approximately by 50\%. For PFPeA, PFHpA, PFDoDA and PFTrDA, the concentrations in the serum pools showed no obvious tendencies for change over time; however, the concentrations of these PFCs were close to the LOQ. The authors conclude that the observed increase in PFOA serum concentrations until the mid-1990s are in accordance with the increasing use of products containing PFCs, while the decreasing concentrations observed the past few years are consistent with the phase-out of these compounds (Haug et al., 2009).

The median concentration of PFOA among 900 Norwegian pregnant women who were part of the Norwegian Mother and Child Cohort Study (enrolled 2003-2004) was $2.2 \mathrm{ng} / \mathrm{mL}$ (Whitworth et al., 2012).

In a study of 60 participants in northern Norway (Andøya Island) during 2005, the relationship between dietary intake and PFC concentrations were investigated (Rylander et al., 2009). Higher concentrations of PFOA (female: $3.4 \mathrm{ng} / \mathrm{ml}$; male: $5.1 \mathrm{ng} / \mathrm{ml}$ ) and PFNA (female: 0.77 $\mathrm{ng} / \mathrm{ml}$; male: $0.94 \mathrm{ng} / \mathrm{ml}$ ) were detected in this population which could be attributable to geographical differences (coastal areas) or dietary habits (Rylander et al., 2009). PFHpA had more than 95\% of the observations below LOD. Higher concentrations of PFOA were found in men. PFNA correlated highly to PFOS.

Rylander and co-workers (Rylander et al., 2010), found a range of PFCAs in 315 middle-aged Norwegian women (48-62 years of age); 
PFOA (4.4 ng/mL) and PFNA (0.81 ng/mL) were detected in more than $90 \%$ of the plasma samples. The concentrations of PFCAs in this study were slightly lower than levels reported from northern Norway (Rylander et al., 2009).

The most recent Norwegian study investigated 19 PFCs in serum from 123 pregnant women collected at the Oslo University hospital during 2007 to 2008 from a sub-cohort of the Norwegian Mother and Child Cohort Study (Gutzkow et al., 2012). Five PFCAs were detected: PFOA (median = $1.12 \mathrm{ng} / \mathrm{ml})$; PFNA (0.34 ng /ml); PFDA (0.07 ng /ml); PFUnDA (0.16 ng / $\mathrm{ml})$; PFTrDA (0.04 ng $/ \mathrm{ml})$. Highly significant correlations $(r>0.60, p<0.001$ ) between most of the PFCs were found with the exception of PFUnDA. The levels of PFCAs in this study were very similar to those reported for 41 female volunteers from the Oslo area in Norway in 2008 (Haug et al., 2011) (Table 9).

The PFC concentrations from 2007-2008 are the lowest reported in Norwegians and lower than those reported in men in 2006 (Haug et al., 2009), probably due to temporal decline in serum levels for many PFCs observed after around year 2000 (Haug et al., 2009) or gender differences, or probably different exposure patterns.

\section{Sweden (SE)}

Several studies have reported the PFAAs level in the general population in Sweden (Glynn et al., 2012; Karrman et al., 2007a; Karrman et al., 2007b; Karrman et al., 2006). The details and PFCA levels are presented in Table 9.

A recent study investigated the temporal trends of blood serum levels of PFCs in primiparous women in the period 1996-2010 living in Uppsala County (Glynn et al., 2012). Among the PFCAs, PFOA, PFNA, PFDA, PFUnDA and PFHpA were detected in the pooled samples, whereas PFHxA, PFDoDA, PFTrDA and PFTeDA were below detection limits (Glynn et al., 2012). During the period 1996-2010 increasing levels were observed for PFNA (4.3\%/year), and PFDA (3.8\%/year), whereas level for PFOA decreased (3.1\%/year). The study suggested that one or several sources of exposure to PFOA have been reduced or eliminated, whereas exposure to the former compounds has recently increased. The serum levels reported in this study are similar to levels found previously in Swedish blood samples by Karrman et al. and other European countries but somewhat lower than reported in the US (Fromme et al., 2009).

Similar to Sweden, increasing levels of PFNA and PFDA were observed in plasma/serum among adults in the US (NHANES) during 1999-2008 (Kato et al., 2011) whereas, among Norwegian men no significant temporal trends of PFNA or PFDA were observed between 1997 and 2007 (Haug et al., 2009). Based on these studies it is not possible to conclude if 
the observed upward trend in Sweden is due to increased exposure to directly emitted PFNA and PFDA, or due to increased emissions of precursor compounds such as fluorotelomer alcohols (Glynn et al., 2012).

\section{Denmark (DK)}

Few biomonitoring studies have been conducted in Denmark measuring the levels of a broad range of PFCs.

A recent study reported the levels of eight different PFCs in serum from young women planning their first pregnancy (collected during 1992-1995) (Vestergaard et al., 2012). Among the women who got pregnant $(\mathrm{n}=129)$, the concentrations of PFOA, PFNA and PFDA were $5.61,0.51$ and $0.11 \mathrm{ng} / \mathrm{ml}$, respectively.

Another study reported the levels of PFOA (and PFOS) in 1,399 maternal blood plasma samples collected during 1996-2002 in Denmark (part of the Danish National Birth Cohort). For the first trimester the mean plasma PFOA levels was $5.6 \mathrm{ng} / \mathrm{ml}$ (Fei et al., 2007; Halldorsson et al., 2008).

Joensen et al. reported the PFC levels in serum samples from young adult males in Denmark $(\mathrm{n}=105)$ collected in 2003 (Joensen et al., 2009). The level of PFOA was $4.9 \mathrm{ng} / \mathrm{ml}$ and the remaining PFCAs (PFDA, PFNA, PFHpA, PFUnA and PFDoA) were found in much lower concentrations with medians ranging from $0.9-0.08 \mathrm{ng} / \mathrm{ml}$. The PFCA levels detected in this study were comparable to those found in Sweden.

The current concentrations of PFCs in Denmark are unknown since the latest biomonitoring data found is from 2003 (Joensen et al., 2009).

\section{Faroe Islands (FO)}

Serum concentrations of 4 PFCAs (PFOA, PFNA, PFDA and PFDoA) were measured in two population groups of whale meat consumers on the Faroe Islands (Weihe et al., 2008).

The first group included 12 mothers sampled in 2000 and their 5 year old children sampled in 2005. The second group consisted of 103 serum samples collected during 1993-1994 of 7-year old children and 79 serum samples of these children at age 14 (collected during 2000-2001) (Weihe et al., 2008).The 5-year old children had higher concentrations of PFOA levels compared to their mothers 5 years previously ( $4.5 \mathrm{ng} / \mathrm{mL}$ vs. $2.4 \mathrm{ng} / \mathrm{ml})$ and PFNA $(1.3 \mathrm{ng} / \mathrm{ml} \mathrm{vs}$. $0.6 \mathrm{ng} / \mathrm{ml}$ ). The concentration of PFDeA was $0.3 \mathrm{ng} / \mathrm{ml}$ for both mothers and children. A decrease was found for PFOA between the 7and 14-year old children ( $5 \mathrm{ng} / \mathrm{ml}$ vs. $4.4 \mathrm{ng} / \mathrm{ml})$, but same PFNA (0.8 $\mathrm{ng} / \mathrm{ml})$ and PFDeA $(0.3 \mathrm{ng} / \mathrm{ml})$ concentrations were found. This suggested a decrease in PFOA during this time period (1993-2001) on 
the Faroe Islands. PFNA concentrations correlated with the frequency of pilot whale consumptions.

On the Faroe Islands, where exposures to marine contaminants via food intake is high, the blood concentrations of PFOA in women were slightly below the average concentrations reported in Danish pregnant women during 1996 to 2002 (Fei et al. 2007), but comparable with those for Swedish women (Glynn et al., 2012).

\section{Greenland (GRL)}

A recent study investigated the level of 10 different PFCs in serum from 284 Inuit belonging to 10 different Greenlandic districts and the temporal trend of blood serum levels of PFCs in Nuuk during 1998-2005 (Long et al., 2012).

The detected PFCAs for Inuit women in this study were PFOA $(2.57$ $\mathrm{ng} / \mathrm{ml})$, PFNA (1.33 ng/ml), PFUnDA (1.23 ng/ml), PFDA $(0.65 \mathrm{ng} / \mathrm{ml})$, PFTrDA $(0.26 \mathrm{ng} / \mathrm{ml})$, PFDoDA $(0.15 \mathrm{ng} / \mathrm{ml})$ and $\mathrm{pFHpA}(0.05 \mathrm{ng} / \mathrm{ml})$. Long et al. reported increasing trends for PFNA (28\%), PFDA (28\%), PFDoA (10\%), PFTrDA (13\%) during 1998-2005; however these trends disappeared upon age adjustment (Long et al., 2012). In this study some correlations between PFCs and legacy POPs (PCBs and organochlorine pesticides) were reported for different non-Nuuk districts. However, for Nuuk Inuit, no significant association was observed between PFCs and legacy POPs, suggesting different sources of exposure other than seafood intake. For non-Nuuk Inuit, significant correlations between serum PFCs and legacy POPs were observed suggesting that there might be common sources for the body burden of PFCs and legacy POPs in non-Nuuk Inuit e.g. marine food intake.

Bonefeld-Jørgensen et al. reported the PFC levels in 115 female Inuit controls from Greenland during 2000-2003, in a study investigating the association of PFCs to breast cancer (Bonefeld-Jorgensen et al., 2011). PFOA (1.63 ng/ml), PFUnA (1.06 ng/ml), PFNA (0.93 ng/ml), PFDA (0.56 $\mathrm{ng} / \mathrm{ml})$, PFDoA $(0.15 \mathrm{ng} / \mathrm{ml})$ and PFTrDA $(0.15 \mathrm{ng} / \mathrm{ml})$ and PFHpA $(0.11 \mathrm{ng} / \mathrm{ml})$ were detected in the healthy control samples.

Another study reported the PFC levels in Greenlandic Inuit men ( $\mathrm{n}=$ 196) from Greenlandic districts, during 2002-2004 (Lindh et al., 2012). The male median concentrations for PFOA $(4.54 \mathrm{ng} / \mathrm{ml})$, PFNA (1.74 $\mathrm{ng} / \mathrm{ml}$ ), PFUnDA (1.28 ng/ml), PFDA (0.87 ng/ml) and PFDoDA (0.14 $\mathrm{ng} / \mathrm{ml}$ ), were comparable to the levels reported for the Inuit women during 2000-2003 (Long et al., 2012) and (Bonefeld-Jorgensen et al., 2011) although the PFOA level was lower for women.

These studies show that the Greenlandic Inuit population is highly exposed to several other, more recently industrially introduced PFCs, 
such as PFNA, PFDA, PFUnA and PFDoA. This indicates a fast distribution of these compounds to the Arctic area. The levels of PFOA found in Inuit men were similar to the levels found in Denmark and other European countries. In contrast, the PFOA level in Inuit women was only approximately 50\% compared to Danish women.

\section{Levels in cord blood}

Four studies were found for the Nordic countries where PFCs were measured in cord blood (Table 10).

In the Norwegian study 19 PFCs were investigated in 123 samples of human maternal and cord blood (2007-2008) and up to 5 different PFCAs (PFOA, PFNA, PFDA, PFUnDA and PFTrDA) were detected (Gutzkow et al., 2012). The median levels in cord blood had the following \% compared to the maternal concentration: for PFOA 79\%; for PFNA $35 \%$; for PFDA $57 \%$ and for PFUnDA $25 \%$, suggesting that PFOA is transferred to the fetus twice as efficiently as the longer-chained PFNA and PFUnDA. Strong correlations between maternal and cord levels of all the tested compounds were found.

A Swedish study compared the maternal levels of PFOA, and PFNA with the levels in cord blood in 19 samples (1996-1999) (Glynn et al., 2012). In cord blood, mean levels of PFOA (1.4 ng/g) and PFNA (estimated to $0.13 \mathrm{ng} / \mathrm{g}$ ) were considerably lower than those in blood serum from the mothers. Significant positive correlations between maternal serum and cord blood levels were found. The strongest correlations between PFC levels in cord and maternal blood were found for maternal serum samples taken during the third trimester, followed by samples taken 3 weeks after delivery.

Another study from the Faroe Islands measured in year 2000 the PFCs in maternal blood, cord blood and breast milk (Needham et al., 2011). Like the other studies they found lower PFC concentrations in cord serum than in maternal serum. PFOA, PFNA, and PFDA revealed good correlation between maternal and cord serum concentrations, with ratios (cord/maternal) of $0.72,0.50$, and 0.29 , respectively. The cord/maternal ratio suggested that the length of PFC chain as well as the active group affected the ability to pass the placenta. PFCs with a short chain length showed higher relative cord serum concentrations than PFCs with a longer chain length. PFCs with sulfonic acid as the active group seemed to pass more easily into the fetal circulation than PFCs with carboxylic acid as the active group (Needham et al., 2010).

In a Danish study PFOA was analyzed in 50 cord blood plasma samples from women in Danish National Birth Cohort (1996-2002) and the results showed mean concentrations of $3.7 \mathrm{ng} / \mathrm{ml}$ for PFOA, which cor- 
responded to $66 \%$ of the level in maternal serum (Fei et al., 2007). Concentrations in cord blood and mother's blood were highly correlated.

The consistent finding of the studies is that cord blood has lower total PFOA than maternal blood; but several PFCs are able to cross the placenta barrier to fetal blood and PFOA seems to cross the placenta most easily. The concentrations of PFOA were highest in Danish and Faroes cord blood probably because the studies are older.

\section{Levels in breast milk}

PFCs have also been found in human milk (see Table 11), but in much lower levels than in blood.

In a Norwegian study of matched samples of serum and breast milk (sampled 2007-2008) up to 11 and 2 PFCs were found in the samples of serum $(n=41)$ and breast milk ( $n=19)$, respectively (Haug et al., 2011). Average median breast milk concentration was $0.025 \mathrm{ng} / \mathrm{ml}$ for PFOA, which corresponded to $3.8 \%$ of the serum concentrations in the mothers.

Thomsen et al. studied the elimination rates of PFOA in breast-milk samples from nine Norwegian mothers living in the Oslo area (Thomsen $e t$ al., 2010). The median concentrations of PFOA in breast milk was 0.05 $\mathrm{ng} / \mathrm{ml}$ and the PFOA breast milk concentration correlated highly (correlation coefficients: 0.99 ) with the mothers serum concentrations. During lactation PFOA concentration in breast milk was reduced by $7.8 \%$ per month, suggesting lactation as an important route of excretion in mothers.

Kärrman et al. (2007) analyzed matched breast milk and serum samples $(\mathrm{n}=12)$ for 7 PFCs during 2004 in Sweden. PFNA and PFOA were detected above detection limits in only one and two milk samples, respectively (Karrman et al., 2007a) .

Sundström et al. measured the concentration of PFOA in pooled human milk samples obtained in Sweden between 1972 and 2008 (Sundstrom et al., 2011). PFOA levels significantly increased from 1972 to 2000 and significantly decreased during 2001-2008. In 2008 the PFOA concentration in the pooled human milk was $0.074 \mathrm{ng} / \mathrm{mL}$. The study showed that the temporal trend in PFOA concentration in pooled human milk samples is similar to the trend in serum concentrations.

A study from the Faroe Islands detected PFOA in breast milk at median concentration $0.1 \mathrm{ng} / \mathrm{ml}$ (collected in 2000) which correlated with the the maternal serum PFOA concentrations $(r=0.80)$ (Needham et al., 2011).

For comparison, a study from China $(n=19)$ reported the presence of PFHpA, PFDA and PFUnDA in human milk from 2004 (Tiido et al., 2006). The concentration of PFOA ranged from 47 to $210 \mathrm{ng} / \mathrm{L}$. The maximum concentrations were $62 \mathrm{ng} / \mathrm{L}$ for PFNA, $15 \mathrm{ng} / \mathrm{L}$ for PFDA, and $56 \mathrm{ng} / \mathrm{L}$ for PFUnDA. 
Although the levels of PFCs in human milk are relatively low compared to the mothers blood level the exposure of these chemicals to the breast fed infant may be significant because of the relatively high exposure per body weight. It is evident that lactation is an exposure pathway as well as a way for maternal excretion.

\section{Levels in Amniotic Fluids}

Only two studies were found measuring PFCAs in human amniotic fluid (Stein et al, 2012) (Bonefeld-Jørgensen, Unpublished data) (Table 12).

In an unpublished Danish study (Bonefeld-Jorgensen et al.) the concentrations of 8 PFCAs were measured in 54 amniotic fluid samples (1995-1999). PFOA, PFHpA and PFDoA were detected in 81.8\%, 1.1\% and $1.1 \%$ of the samples, respectively. The average PFOA level was $0.37-$ $0.29 \mathrm{ng} / \mathrm{ml}$ (Bonefeld-Jorgensen et al. manuscript in preparation).

Using paired samples from 28 women from the US collected in 20052008, the concentrations of 3 PFCAs (PFOA, PFNA, and PFDeA) were measured in serum and amniotic fluid (second trimester) (Stein, 2012). The detected carboxylates were PFOA $(0.3 \mathrm{ng} / \mathrm{ml}$ detected in $24 \mathrm{sam}-$ ples) and PFNA (0.2 ng/ml detected in 10 samples). PFOA showed weaker correlations between serum and amniotic fluid $(\rho=0.64)$. Amniotic fluid concentrations are lower than maternal blood (10-20 fold) and considerably lower than cord blood concentrations. The PFCs detected in the US were lower than those reported from Denmark, probably due to the older Danish samples (1980-1990). PFOA appeared to be more soluble than PFOS because it was detected in amniotic fluid at lower maternal serum levels than PFOS.

\subsubsection{Conclusions on human biomonitoring of PFCAs}

In the general population the level of PFOA has been increasing until mid-1990s and has then decreased in human serum since 2002. However, for PFPeA, PFHpA, PFDoDA, and PFTrDA no obvious tendencies have been observed (in Norway). In most studies PFOA, PFNA, PFDA, PFUnDA and PFHpA have been detected in human blood whereas PFHxA, PFDoDA, PFTrDA, PFTeDA have been below detection limits. In general, the blood levels are higher in males.

Intake of fish, shellfish, and whale were in some studies identified as determinants of serum concentrations. However, other factors, such as consumer products and indoor air (e.g., house dust in carpeted houses) were also identified to contribute to PFC exposure.

In the Faroe Islands data for 7 and 14-years children indicate a decreasing trend for PFOA during 1993-2003. 
In general, comparable levels were observed for the Nordic countries although the newest and lowest levels were found for Sweden and Norway.

Of PFCAs mainly PFOA, and to some extent PFNA and PFDA, were detected in cord blood, but the concentrations are usually lower than concentrations observed in maternal serum or plasma, although the maternal and cord blood data are highly correlated. PFCAs with longer chains are transferred less efficiently to the fetus than those with shorter chain. Detection of PFCAs in cord blood means that some of the compounds can cross the placental barrier and the fetus is prenatally exposed to these compounds.

Of PFCAs only PFOA was detected in breast milk from women in Nordic countries and the concentrations in milk are 3-4\% of what is found in the corresponding serum concentrations (Haug et al., 2011). For comparison, in China PFNA, PFDA and PFUnDA in addition to PFOA, were also detected in some samples.

Monitoring studies of PFCAs in amniotic fluids are scarce, but a Danish study detected PFOA and PFNA in amniotic fluids at concentrations 10-20 fold lower than in maternal blood.

FTOHs are identified to be metabolized to PFOA and are thus a source of PFCAs, and an indirect exposure via fluorotelomer-based commercial products or residuals can explain continued exposure to PFOA, together with exposure to PFNA and PFDA, without similar exposure to PFOS.

Further research is therefore needed to determine whether the constant or slowly increasing concentrations of long-chain PFCAs in human serum are primarily a consequence of ongoing exposure to telomerbased precursors. 
Table 9. Median concentrations (in $\mathrm{ng} / \mathrm{mL}$ ) of PFCAs and PFSAs in blood (serum /plasma) from Nordic countries

\begin{tabular}{|c|c|c|c|c|c|c|c|c|c|c|c|c|c|c|c|c|c|c|c|c|c|c|c|}
\hline Reference & $\begin{array}{l}\text { Coun- } \\
\text { try }\end{array}$ & $\begin{array}{l}\text { Samp. } \\
\text { Year }\end{array}$ & $n$ & Sex & Age & Matrix & 畚 & $\begin{array}{l}\frac{\tilde{x}}{x} \\
\frac{1}{a} \\
\end{array}$ & $\begin{array}{l}\frac{n}{2} \\
\frac{\underline{T}}{a} \\
\underline{a}\end{array}$ & 号 & 幾 & 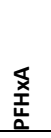 & 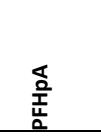 & 兘 & 竞 & 혐 & 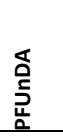 & 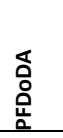 & 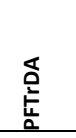 & $\begin{array}{l}\frac{\sigma}{d} \\
\text { ț } \\
\end{array}$ & 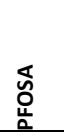 & 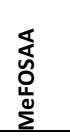 & 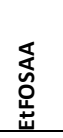 \\
\hline $\begin{array}{l}\text { Vestergaard } \\
\text { (2012) }\end{array}$ & DK & $\begin{array}{l}1992- \\
1995\end{array}$ & 129 & $\mathrm{~F}$ & 27 & s & & 1.22 & & 36.3 & & & & 5.61 & 0.51 & 0.11 & & & & & 0.11 & 0.39 & 1.79 \\
\hline $\begin{array}{l}\text { Eriksen } \\
\text { (2011) }\end{array}$ & DK & $\begin{array}{l}1993- \\
1997\end{array}$ & 652 & M & 55 & $\mathrm{P}$ & & & & 34.9 & & & & 6.80 & & & & & & & & & \\
\hline $\begin{array}{l}\text { Fei } * \\
(2007)\end{array}$ & DK & $\begin{array}{l}1996- \\
2002\end{array}$ & 1,399 & $\mathrm{~F}$ & ? & $\mathrm{s}$ & & & & 35.3 & & & & 5.60 & & & & & & & & & \\
\hline $\begin{array}{l}\text { Joensen } \\
\text { (2009) }\end{array}$ & DK & 2003 & 105 & M & $18-19$ & $\mathrm{~s}$ & & 6.60 & & 24.5 & & & 0.20 & 4.90 & 0.80 & 0.90 & 0.10 & 0.08 & $0-0.2$ & & 0.06 & & \\
\hline $\begin{array}{l}\text { Haug } \\
\text { (2009) }\end{array}$ & NO & $2001^{\#}$ & pool & M & $40-50$ & $\mathrm{~s}$ & $<0.05$ & 1.60 & 0.10 & 27.00 & & & 0.08 & 4.90 & 1.20 & 0.25 & 0.24 & 0.05 & 0.16 & & 0.15 & & \\
\hline $\begin{array}{l}\text { Haug } \\
\text { (2010) }\end{array}$ & NO & 2003 & 175 & $M+F$ & $57+55$ & $\mathrm{P}$ & & 1.70 & 0.42 & 25.0 & & & $<0.035$ & 3.60 & 0.90 & 0.35 & 0.47 & 0.06 & 0.09 & & 0.22 & & \\
\hline $\begin{array}{l}\text { Rylander } \\
\text { (2010) }\end{array}$ & NO & 2004 & 326 & $\mathrm{~F}$ & 56 & P & & 1.00 & 0.32 & 20.0 & & & nd & 4.40 & 0.81 & & & & & & 0.02 & & \\
\hline $\begin{array}{l}\text { Haug } \\
\text { (2009) }\end{array}$ & NO & $2004^{\#}$ & pool & M & $40-50$ & $\mathrm{~s}$ & $<0.05$ & 1.40 & 0.12 & 18.0 & $<0.05$ & & 0.14 & 3.40 & 0.78 & 0.31 & 0.18 & 0.06 & 0.11 & & 0.05 & & \\
\hline $\begin{array}{l}\text { Rylander } \\
\text { (2009) }\end{array}$ & NO\# & 2005 & 15 & M & 44 & P & & 1.80 & 0.70 & 43.0 & & & nd & 5.10 & 0.94 & & & & & & 0.11 & & \\
\hline
\end{tabular}




\begin{tabular}{|c|c|c|c|c|c|c|c|c|c|c|c|c|c|c|c|c|c|c|c|c|c|c|c|}
\hline Reference & $\begin{array}{l}\text { Coun- } \\
\text { try }\end{array}$ & $\begin{array}{l}\text { Samp. } \\
\text { Year }\end{array}$ & $n$ & Sex & Age & Matrix & 兽 & $\begin{array}{l}\tilde{\underline{x}} \\
\text { 章 } \\
\end{array}$ & 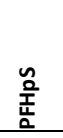 & 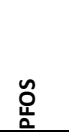 & 厸 & 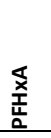 & $\begin{array}{l}\frac{\sigma}{\frac{\alpha}{a}} \\
\frac{1}{a} \\
\end{array}$ & $\begin{array}{l}\overleftarrow{d} \\
\frac{1}{2} \\
\end{array}$ & $\sum_{\frac{\pi}{a}}^{\overleftarrow{a}}$ & 현 & 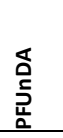 & 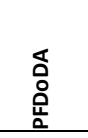 & $\begin{array}{l}\text { 总 } \\
\text { 悹 }\end{array}$ & 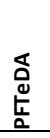 & 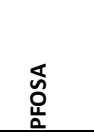 & 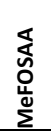 & 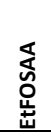 \\
\hline $\begin{array}{l}\text { Rylander } \\
\text { (2009) }\end{array}$ & NO\# & 2005 & 41 & $\mathrm{~F}$ & 44 & $P$ & & 0.80 & 0.35 & 24.0 & & & nd & 3.40 & 0.77 & & & & & & 0.08 & & \\
\hline $\begin{array}{l}\text { Haug } \\
\text { (2009) }\end{array}$ & NO & $2006^{\#}$ & pool & M & $40-51$ & $\mathrm{~s}$ & $<0.05$ & 1.40 & 0.06 & 12.0 & $<0.05$ & & 0.08 & 2.70 & 0.55 & 0.22 & 0.14 & 0.05 & 0.07 & & 0.05 & & \\
\hline $\begin{array}{l}\text { Haug } \\
\text { (2011) }\end{array}$ & NO & $\begin{array}{l}2007- \\
2008\end{array}$ & 41 & $\mathrm{~F}$ & 37 & $\mathrm{~s}$ & & 0.39 & 0.08 & 6.7 & & & $<0.035$ & 1.40 & 0.63 & 0.23 & 0.42 & $<0.035$ & $<0.035$ & & $<0.035$ & & \\
\hline $\begin{array}{l}\text { Gützkow } \\
\text { (2012) }\end{array}$ & NO & $\begin{array}{l}2007- \\
2008\end{array}$ & 123 & $\mathrm{~F}$ & & $\mathrm{P}$ & & 0.28 & & 5.0 & & & & 1.12 & 0.34 & 0.07 & 0.16 & & 0.04 & & & & \\
\hline $\begin{array}{l}\text { Glynn A } \\
\text { (2012) }\end{array}$ & SE & 1996 & pool & $\mathrm{F}$ & 30 & s & 0.02 & 2.24 & & 23.3 & 0.26 & nd & 0.08 & 2.69 & 0.50 & 0.21 & 0.16 & nd & nd & nd & 0.51 & & \\
\hline $\begin{array}{l}\text { Kärrman } \\
\text { (2006) }\end{array}$ & $\mathrm{SE}$ & $\begin{array}{l}1997- \\
1999\end{array}$ & 40 & M & & WB & & 1.70 & & 17.7 & $\mathrm{Nd}$ & & & 2.70 & 0.30 & 0.10 & 0.20 & & & & 2.70 & & \\
\hline $\begin{array}{l}\text { Kärrman } \\
\text { (2006) }\end{array}$ & $\mathrm{SE}$ & $\begin{array}{l}1997- \\
2000\end{array}$ & 26 & $\mathrm{~F}$ & & WB & & 1.20 & & 16.9 & & & & 2.10 & 0.30 & 0.20 & 0.10 & & & & 2.70 & & \\
\hline $\begin{array}{l}\text { Kärrman } \\
\text { (2004) }\end{array}$ & SE & $\begin{array}{l}1997- \\
2000\end{array}$ & 66 & $\mathrm{~F}$ & 19-75 & S & & 3.00 & & 34.2 & & & & 5.00 & & & & & & & & & \\
\hline $\begin{array}{l}\text { Glynn A } \\
\text { (2012) }\end{array}$ & $\mathrm{SE}$ & 2000 & Pool & $\mathrm{F}$ & 30 & $\mathrm{~s}$ & $<0.01$ & 3.03 & & 22.0 & 0.05 & nd & 0.09 & 2.50 & 0.38 & 0.17 & 0.20 & nd & nd & nd & 0.44 & & \\
\hline $\begin{array}{l}\text { Kärrman } \\
\text { (2007) }\end{array}$ & SE & 2004 & 12 & $\mathrm{~F}$ & & $\mathrm{~s}$ & - & 4.00 & - & 18.7 & $\begin{array}{c}1 \\
\text { sample }\end{array}$ & nd & nd & 3.80 & 0.63 & 0.43 & 0.28 & nd & & - & 0.19 & & \\
\hline
\end{tabular}




\begin{tabular}{|c|c|c|c|c|c|c|c|c|c|c|c|c|c|c|c|c|c|c|c|c|c|c|c|}
\hline Reference & $\begin{array}{l}\text { Coun- } \\
\text { try }\end{array}$ & $\begin{array}{l}\text { Samp. } \\
\text { Year }\end{array}$ & $n$ & Sex & Age & Matrix & 兽 & $\begin{array}{l}\frac{\tilde{x}}{\underline{x}} \\
\frac{1}{a} \\
\end{array}$ & 鵕 & 品 & 吕 & 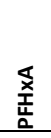 & $\begin{array}{l}\text { 亮 } \\
\text { 盖 } \\
\end{array}$ & $\begin{array}{l}\overleftarrow{\delta} \\
\text { 임 } \\
\end{array}$ & $\sum_{\frac{\pi}{a}}^{\overleftarrow{a}}$ & 염 & $\begin{array}{l}\text { 竞 } \\
\text { 总 } \\
\end{array}$ & 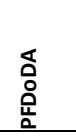 & $\begin{array}{l}\text { 员 } \\
\text { 晏 }\end{array}$ & $\begin{array}{l}\text { 离 } \\
\text { 音 } \\
\end{array}$ & $\begin{array}{l}\text { 氐 } \\
\text { 움 }\end{array}$ & 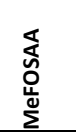 & $\begin{array}{l}\text { 委 } \\
\text { ò } \\
\text { 焉 } \\
\end{array}$ \\
\hline $\begin{array}{l}\text { Jönsson } \\
2010\end{array}$ & SE & 2009 & 50 & M & & $s$ & & 0.78 & & 6.9 & & & & 1.9 & 0.96 & 0.41 & $<\angle O D$ & & & & & & \\
\hline $\begin{array}{l}\text { Glynn A } \\
\text { (2012) }\end{array}$ & $\mathrm{SE}$ & 2010 & Pool & $\mathrm{F}$ & 30 & $\mathrm{~s}$ & 0.10 & 7.95 & & 7.6 & 0.01 & $\mathrm{nd}$ & 0.08 & 1.39 & 0.59 & 0.28 & 0.19 & nd & nd & nd & $<0.040$ & & \\
\hline $\begin{array}{l}\text { Nilsson H } \\
\text { (2010) }\end{array}$ & $\begin{array}{l}\text { SE (ski- } \\
\text { wax) }\end{array}$ & $\begin{array}{l}2007- \\
2008\end{array}$ & 8 & M & 36 & WB & $\mathrm{Nd}$ & 1.64 & & 12.2 & & nd & 2.80 & 112 & 14.7 & 7.90 & $0.1-2.8$ & & & & & & \\
\hline $\begin{array}{l}\text { Weihe P } \\
\text { (2008) }\end{array}$ & $\begin{array}{l}\text { FO } \\
\text { (Faro- } \\
\text { es) }\end{array}$ & $\begin{array}{l}1993- \\
1994\end{array}$ & 103 & Child & 7 & $\mathrm{~s}$ & & 0.40 & & 26.3 & & & & 5.00 & 0.80 & 0.30 & & & & & 1.30 & 0.40 & 1.40 \\
\hline $\begin{array}{l}\text { Needham } \\
\text { (2010) }\end{array}$ & FO & 2000 & 12 & $\mathrm{~F}$ & & $\mathrm{~s}$ & & 12.30 & & 19.7 & & & & 4.20 & 0.76 & 0.34 & & & & & & & \\
\hline $\begin{array}{l}\text { Weihe P } \\
\text { (2008) }\end{array}$ & FO & 2000 & 12 & $\mathrm{~F}$ & & $\mathrm{~s}$ & & $\begin{array}{c}0.6 \\
<\angle O D\end{array}$ & & 23.7 & & & & 2.40 & 0.60 & 0.30 & & & & & 0.60 & 0.90 & $\begin{array}{c}0.2 \\
<L O D\end{array}$ \\
\hline $\begin{array}{l}\text { Weihe P } \\
\text { (2008) }\end{array}$ & FO & $\begin{array}{l}2000- \\
2001\end{array}$ & 79 & Child & 14 & $\mathrm{~s}$ & & 2.90 & & 31.2 & & & & 4.20 & 0.80 & 0.30 & & & & & 0.30 & 0.40 & 1.00 \\
\hline $\begin{array}{l}\text { Weine P } \\
\text { (2008) }\end{array}$ & FO & 2005 & 12 & Child & 5 & $\mathrm{~s}$ & & 0.60 & & 16.3 & & & & 4.50 & 1.30 & 0.30 & & & & & $<\angle O D$ & 0.30 & $\begin{array}{c}0.2 \\
(<L O D)\end{array}$ \\
\hline $\begin{array}{l}\text { Long M } \\
\text { (2012) }\end{array}$ & $\begin{array}{l}\text { GRL } \\
\text { (Nuuk) }\end{array}$ & $\begin{array}{l}1998- \\
2005\end{array}$ & 5 & M & 65 & $\mathrm{~s}$ & & 8.50 & & 74.5 & & & 0.52 & 7.19 & 5.59 & 3.13 & 7.32 & 0.80 & 1.39 & & 0.50 & & \\
\hline $\begin{array}{l}\text { Long M } \\
\text { (2012) }\end{array}$ & $\begin{array}{l}\text { GRL } \\
\text { (All) }\end{array}$ & $\begin{array}{l}1998- \\
2005\end{array}$ & 209 & $\mathrm{~F}$ & 53 & $\mathrm{~s}$ & & 2.50 & & 28.5 & & & 0.05 & 2.57 & 1.33 & 0.65 & 1.23 & 0.15 & 0.26 & & 0.20 & & \\
\hline
\end{tabular}




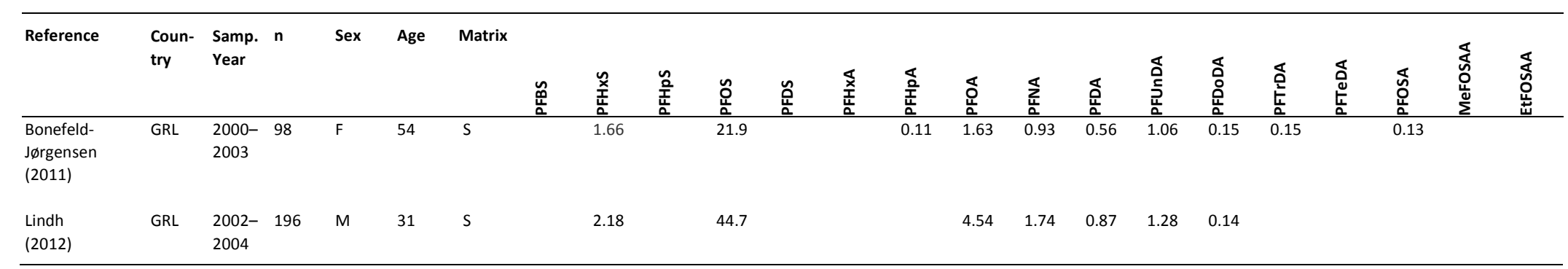

* Mean concentrations; " Pooled serum samples; F: female; M: male; S: serum; P: plasma; WB: whole blood; LOD: limit of detection; nd: not detecte. 
Table 10. Median concentrations (in $\mathrm{ng} / \mathrm{mL}$ ) of PFCAs and PFSAs in cord blood

\begin{tabular}{|c|c|c|c|c|c|c|c|c|c|c|c|c|c|c|c|c|c|c|}
\hline \multirow[b]{2}{*}{ Reference } & \multirow[b]{2}{*}{ Country } & \multirow[b]{2}{*}{ Sample year } & \multirow[b]{2}{*}{$\mathbf{N}$} & \multirow[b]{2}{*}{ Matrix } & \multicolumn{7}{|c|}{ PFSAs (ng/ml) } & \multicolumn{7}{|c|}{ PFCAs (ng/ml) } \\
\hline & & & & & 兽 & $\begin{array}{l}\underline{\tilde{x}} \\
\underline{\underline{\underline{x}}} \\
\end{array}$ & $\begin{array}{l}\text { 옴 } \\
\text { 픔 }\end{array}$ & 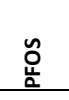 & 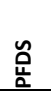 & $\begin{array}{l}\frac{\sqrt{x}}{\mathbf{x}} \\
\text { 音 } \\
\end{array}$ & 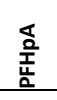 & $\begin{array}{l}\text { do } \\
\text { a } \\
\end{array}$ & $\underset{\underline{a}}{\frac{\pi}{a}}$ & 递 & $\begin{array}{l}\text { 品 } \\
\text { 훔 } \\
\end{array}$ & 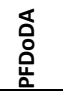 & $\begin{array}{l}\text { 委 } \\
\text { 点 } \\
\end{array}$ & 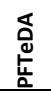 \\
\hline Fei C (2007)* & Denmark & 1996-2002 & 50 & Serum & & & & 11 & & & & 3.7 & & & & & & \\
\hline Needham (2010) & Faroe Island & 2000 & 12 & Serum & & 9.1 & & 6.6 & & & & 3.1 & 0.37 & 0.1 & & & & \\
\hline Glynn A (2012) * & Sweden & 1996-2010 & 19 & WB (ng/g) & & & & 5.3 & & & & 1.4 & 0.13 & & & & & \\
\hline Gützkow (2012) & Norway & $2007-2008$ & 123 & Serum & ND & 0.2 & ND & 1.52 & ND & ND & ND & 0.88 & 0.12 & 0.04 & 0.04 & ND & 0.04 & ND \\
\hline Fromme (2010) & Germany & 2008-2009 & 33 & Serum & ND & 0.2 & & 1 & & & & 1.4 & $<0.4$ & $<0.4$ & & ND & & \\
\hline
\end{tabular}

* Mean; WB: Whole blood; ND: not detected.

Table 11. Median concentrations (in $\mathrm{ng} / \mathrm{ml}$ ) of PFCAs and PFSAs in breast milk

\begin{tabular}{|c|c|c|c|c|c|c|c|c|c|c|c|c|c|c|c|c|c|}
\hline \multirow[b]{2}{*}{ Reference } & \multirow[b]{2}{*}{ Country } & \multirow[b]{2}{*}{ Sample year } & \multirow[b]{2}{*}{$\mathrm{n}$} & \multicolumn{7}{|c|}{ PFSAs (ng/ml) } & \multicolumn{7}{|c|}{ PFCAs (ng/ml) } \\
\hline & & & & 幾 & $\begin{array}{l}\frac{\tilde{x}}{x} \\
\frac{x}{a} \\
\end{array}$ & 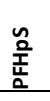 & 吕 & 㟧 & $\begin{array}{l}\frac{\sqrt{x}}{x} \\
\text { 章 }\end{array}$ & $\begin{array}{l}\frac{\Phi}{\frac{\alpha}{2}} \\
\frac{T}{\underline{L}}\end{array}$ & $\begin{array}{l}\text { ó } \\
\text { 몀 }\end{array}$ & $\sum_{\frac{u}{a}}^{\mathbb{a}}$ & 道 & 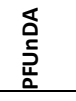 & $\begin{array}{l}\text { 号 } \\
\text { 믐 }\end{array}$ & $\begin{array}{l}\text { 亮 } \\
\text { 点 }\end{array}$ & 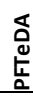 \\
\hline Kärrman (2007) & Sweden & 2004 & 12 & & 0.07 & & 0.166 & & & & nd & $2 \mathrm{sampl}$ & nd & nd & & & \\
\hline Haug (2011) & Norway & $2007-2008$ & 19 & nd & nd & nd & 0.087 & nd & nd & nd & 0.025 & nd & nd & nd & nd & nd & \\
\hline Thomsen (2010) & Norway & 2001-2009 & 68 & & nd & nd & 0.11 & & & & 0.05 & nd & nd & & & & \\
\hline Needham (2010) & Faroes & 2000 & 12 & & & & & & & & 0.1 & & & & & & \\
\hline Sundström (2011) & Sweden & 2008 & 1 pool & & 0.014 & & 0.075 & & & & 0.074 & & & & & & \\
\hline Liu J (2011) & China (Jinhu) & 2009 & 50 & & nd & & 0.042 & & & & 0.121 & 0.019 & 0.017 & 0.024 & nd & & \\
\hline
\end{tabular}


Table 12. Median concentrations (in $\mathrm{ng} / \mathrm{ml}$ ) of PFCAs and PFSAs in amniotic fluids

\begin{tabular}{|c|c|c|c|c|c|c|c|c|c|c|c|c|c|c|c|c|c|}
\hline \multirow[b]{2}{*}{ Reference } & \multirow[b]{2}{*}{ Country } & \multirow[b]{2}{*}{ Year } & \multirow[b]{2}{*}{$\mathrm{n}$} & \multicolumn{7}{|c|}{ PFSAs (ng/ml) } & \multicolumn{7}{|c|}{ PFCAs (ng/ml) } \\
\hline & & & & 兽 & $\begin{array}{l}\frac{\tilde{x}}{\underline{x}} \\
\frac{\underline{x}}{a} \\
\end{array}$ & 咮 & 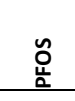 & 气̆ & $\begin{array}{l}\frac{x}{\vec{x}} \\
\text { 章 } \\
\end{array}$ & $\begin{array}{l}\frac{d}{2} \\
\frac{1}{14} \\
\end{array}$ & 임 & $\sum_{\frac{\pi}{a}}^{\mathbb{a}}$ & 迸 & $\begin{array}{l}\text { 号 } \\
\text { D } \\
\text { 믐 }\end{array}$ & $\begin{array}{l}\text { 品 } \\
\text { 믐 } \\
\end{array}$ & $\begin{array}{l}\frac{5}{0} \\
\frac{1}{a}\end{array}$ & $\begin{array}{l}\overleftarrow{\Delta} \\
\frac{a}{a} \\
\underline{a}\end{array}$ \\
\hline Jensen (2012) & DK & 1980-1996 & 300 & & & & 1.1 & & & & & & & & & & \\
\hline Bonefeld-Jørgensen (unpublished) ${ }^{1}$ & DK & 1995-1999 & 51 & & $\begin{array}{l}0.065 \\
(4.5 \%)\end{array}$ & & $\begin{array}{c}1.44 \\
(47 \%)\end{array}$ & & & & $\begin{array}{c}0.32 \\
(82 \%)\end{array}$ & & & & $\begin{array}{c}0.205 \\
(1 \%)\end{array}$ & & \\
\hline Stein $(2012)^{2}$ & US (NY) & $2005-2008$ & 28 & & 0.4 & & 0.4 & & & & 0.3 & 0.2 & ND & & & & \\
\hline
\end{tabular}

${ }^{1}$ Bonefeld-Jørgensen: PFOS, PFOSA, PFOA, PFHxS, PFHpA and PFD oA could be detected in $46.6 \% .35 .2 \% .81 .8 \% .4 .5 \% .1 .1 \%$ and $1.1 \%$ of the AF samples. The rest were below detection limit.

${ }^{2}$ Stein: PFOA was detected in 24/28.; PFOS in 9/28 and PFDA only in 1 sample. 


\subsubsection{PFSAs (perfluoroalkyl sulfonates) in humans}

\section{Levels in blood}

Blood levels of PFSAs have been monitored in some Nordic countries and usually PFOS and PFHxS are detected most frequently, with PFOS having the highest concentrations. Since in many studies both PFCAs and PFSAs were measured the study design of many of the studies are already mentioned under the section for PFCAs.

In the following the monitoring data for PFSAs are described for each Nordic country and data presented in Table 9.

\section{Norway}

Time trends of 19 different PFCs for the general Norwegian population were investigated in a cross sectional study by Haug and coworkers (Haug et al., 2009). PFOS was found in highest concentrations in all samples, followed by PFHxS. The concentrations in 2006 for PFOS and PFHxS were found to be 12 and $1.4 \mathrm{ng} / \mathrm{mL}$ serum, respectively. PFHpS were detected in most samples, while PFBS were found less frequently. PFDS were not observed above the LOQ in any of the samples (Haug et al., 2009).

The pooled serum samples showed an up to 9-fold increase of PFOS and PFHpS from 1976 to the mid-1990s. Between 2000 and 2006, PFOS levels decreased by approximately 50\%, while PFHxS level decreased by 90\%. In this study, PFOS and PFOA were significantly correlated to each other as well as to PFHxS, PFHpS, PFNA, PFDA, and PFTrDA. Correlations between the PFCAs, e.g. PFOA, and PFSAs, e.g. PFOS, indicate common sources for human exposure to these two PFC classes, as they cannot convert directly into each other (Haug et al., 2009).

In a study in northern Norway (Andøya Island) during 2005 slightly higher concentrations of plasma PFOS (female: $24 \mathrm{ng} / \mathrm{ml}$; male: 43 $\mathrm{ng} / \mathrm{ml}$ ), PFHxS (female: $0.8 \mathrm{ng} / \mathrm{ml}$; male: $1.8 \mathrm{ng} / \mathrm{ml}$ ) and PFHpS (female: $0.35 \mathrm{ng} / \mathrm{ml}$; male: $0.7 \mathrm{ng} / \mathrm{ml}$ ) were found among coastal population compared to the general Norwegian population (Rylander et al., 2009). Men had higher concentrations of the PFSAs (including PFOS) but lower proportions of linear PFOS compared to women. The linear isomer of PFOS is most common in technical mixtures and also in human samples and difference in proportions could indicate different exposure sources (Karrman et al., 2007b; Rylander et al., 2009). High correlation was found between PFOS and PFHpS ( $r=0.93)$. The PFOS and PFHpS concentrations decreased with intake of fruits and vegetables, whereas an increase was observed with intake of fatty fish (Rylander et al., 2009). 
Intake of fatty fish and intake of fruits and vegetables were not correlated in this study, and therefore the effect of extra intake of fruit could not be fully explained by the authors (Rylander et al., 2009).

In the most recent Norwegian study measuring the PFCs in serum from 123 pregnant women from Oslo during 2007-2008 (Gutzkow et al., 2012), the PFOS and PFHxS were detected at 5 and $0.28 \mathrm{ng} / \mathrm{ml}$, respectively, and was the lowest reported in Norwegians and other Nordic countries. This could indicate a decline in serum levels or different pattern of exposure for this sub-cohort of the Norwegian Mother and Child Cohort Study.

\section{Sweden}

Several studies have reported the PFSAs level in the general population in Sweden (Glynn et al., 2012; Karrman et al., 2007a; Karrman et al., 2007b; Karrman et al., 2006).

A study investigated the temporal trends of blood serum levels of PFCs in pregnant women during 1996-2010 living in the Uppsala County (Glynn et al., 2012). Increasing levels were observed for PFBS and PFHxS, whereas levels for PFOS and PFDS were decreased. The serum concentrations of the PFOS, PFHxS, PFBS and PFDS in the pooled samples from 2010 were 7.6, 7.95, 0.1 and $0.01 \mathrm{ng} / \mathrm{ml}$ respectively. In 2010, PFHxS levels did reach those of PFOS that indicates that the Swedish women have recently been exposed to increasing levels of PFHxS-related compounds from sources that are independent from PFOS exposure (Glynn et al., 2012).

In overall the Swedish studies show that the phase-out of PFOSrelated chemicals has resulted in decreasing serum concentrations of PFOS in the blood serum of young Swedish women during the past decade. However, exposure to sulfonates with shorter carbon chains than PFOS (or their respective precursors) is currently increasing.

\section{Denmark}

For young Danish women planning their first pregnancy (serum collected during 1992-1995), the concentrations of PFOS and PFHxS were 36.3 $\mathrm{ng} / \mathrm{ml}$ and $1.22 \mathrm{ng} / \mathrm{ml}$, respectively (Vestergaard et al., 2012). The median serum levels were similar to most levels reported for U.S. populations during this period.

Comparable PFOS plasma concentrations of $35.1 \mathrm{ng} / \mathrm{ml}$ were reported for 1,399 pregnant women during 1996-2002 in Denmark (part of the Danish National Birth Cohort) (Fei et al., 2007; Halldorsson et al., 2008).

For young adult Danish men in 2003, the median PFOS and PFHxS concentrations were 24.5 and $6.6 \mathrm{ng} / \mathrm{mL}$, respectively (Joensen et al., 
2009), and PFOSA was detected in only 56 men (median $0.06 \mathrm{ng} / \mathrm{ml}$ ). The PFSA levels detected in this study were comparable with those found in other countries such as Sweden (Karrman et al., 2007a), but lower than results from the women in Denmark (Fei et al., 2007).

\section{Faroe Islands}

In paired mother (sampled in 2000) and 5-year old child (sampled in 2005) samples from the whale consuming Faroe Island population, the median concentration of PFOS was 23.7 and $16.3 \mathrm{ng} / \mathrm{mL}$ in the mothers and children, respectively (Weihe et al., 2008). The PFHxS was detected in only $25 \%$ of the maternal samples but in all the children (median $0.6 \mathrm{ng} / \mathrm{ml}$ ).

In samples from children at age 7 (1993-1994) and again at age 14 (2000-2001), the median concentration increased for PFOS from 26.3 $\mathrm{ng} / \mathrm{ml}$ to $31.2 \mathrm{ng} / \mathrm{ml}$, and for PFHxS a 3-fold increase was seen in the PFHxS ( $\mathrm{p}<0.001)$ concentrations (Weihe et al., 2008).

Overall, the concentrations of PFOS for the Faroe Island women are slightly below average concentrations reported in Danish pregnant women (Fei et al., 2007). Traditional whale meat consumption was suggested to be a major contributor to PFOS and PFNS exposures, while fish intake was associated to $\mathrm{PFHxS}$ concentrations (Weihe et al., 2008).

\section{Greenland}

Long et al. investigated the levels of 10 different PFCs in serum from 284 Greenlandic Inuit during 1998-2005 (Long et al., 2012). The median concentrations of PFOS and PFHxS in Inuit females were 28.5 and 2.5 $\mathrm{ng} / \mathrm{ml}$ respectively. No time trends were found for PFSAs, but men had higher PFSA concentrations. The observed serum PFSA concentrations in Inuits corresponded to the range observed in European biomonitoring studies of the general population (Fromme et al., 2009).

In another study of female Greenlandic Inuit from 2000-2003, the mean concentrations of PFOS and PFHxS were measured to be 21.9 and $1.66 \mathrm{ng} / \mathrm{ml}$ respectively (Bonefeld-Jorgensen et al., 2011), and lower than those reported by Long and co-workers (Long et al., 2012). This could be due to geographical differences as district differences was observed and also that non-Nuuk Inuit women had significantly lower PFC levels than Inuit women from Nuuk.

For Greenlandic men, the median concentrations of PFOS and PFHxS in 2002-2004 were reported to be 44.7 and $2.18 \mathrm{ng} / \mathrm{ml}$ (Lindh et al., 2012). Seafood was one of the determinants of PFOS. The level of PFOS reported in this study was higher than those reported by Long et al (14.9 
$\mathrm{ng} / \mathrm{ml}$ ). However, again geographical differences between the different districts in Greenland was observed e.g. Long et al found for men in Nuuk (median, $74.5 \mathrm{ng} / \mathrm{ml}$ ) and non-Nuuk (median, $13.0 \mathrm{ng} / \mathrm{ml}$ ).

\section{Levels in cord blood}

The studies are also described under the PFCA section above and summarized in Table 10.

In the Norwegian study of 123 samples of human maternal and cord blood PFHxS and PFOS were detected in cord blood at 0.23 and 1.52 $\mathrm{ng} / \mathrm{ml}$, respectively (Gutzkow et al., 2012). The median levels in cord blood had the following \% compared to the maternal concentration: PFHXS 70\%; for PFOS 30\% suggesting that PFHxS is transferred to the fetus much more efficiently than PFOS.

The Swedish study of 19 samples collected in 1996-1999 detected PFOS (5.3 ng/g whole blood) in the cord blood, which was considerably lower than those found in blood serum from the mothers. Significantly positive correlations between maternal serum and cord blood levels were found (Glynn et al., 2012).

The mean concentration of PFOS in 50 cord blood plasma samples from the Danish National Birth Cohort (1996-2002) was $11 \mathrm{ng} / \mathrm{ml}$, corresponding to $30 \%$ of the level in maternal serum (Fei et al., 2007).

The study from the Faroe Islands in 2000 (Needham et al., 2010) found PFOS and PFHxS at 6.60 and $9.10 \mathrm{ng} / \mathrm{ml}$, respectively in the cord blood, which correlated well with the concentrations in maternal blood, with ratios (cord/maternal) of 0.34 and 0.74 . The cord/maternal ratio suggested that the length of PFC chain as well as the active group affected the ability to pass the placenta. PFCs with a short chain length showed higher relative cord serum concentrations than PFCs with a longer chain length. PFCs with sulfonic acid as the active group seemed to pass more easily into the fetal circulation than PFCs with carboxylic acid as the active group (Needham et al., 2010).

Overall, the studies found correlations between PFSAs in maternal and cord serum, with lower PFSA concentrations in cord serum than in maternal serum. The passage to fetal blood is easier for PFHxS.

\section{Levels in breast milk}

The results are summarized in Table 11.

Kärrman et al. (2007) analyzed matched breast milk and serum samples $(\mathrm{n}=12)$ during 2004 in Sweden. Of the eight PFASs found in the serum samples, five were detected in the matched milk samples. PFOS and PFHxS were detected in all milk samples at median concentrations of $0.166 \mathrm{ng} / \mathrm{mL}$ and $0.07 \mathrm{ng} / \mathrm{mL}$, respectively. Similar PFC occurrence 
and levels were found in the milk composite samples collected between 1996 and 2004. The mean ratios between milk and serum (M:S) concentrations were 0.01:1 for PFOS and 0.02:1 for PFHxS. The serum and milk pattern suggested that PFHxS is excreted to milk in a higher degree than PFOS. In 2008, measured concentrations of PFOS and PFHxS in pooled human milk were $0.075 \mathrm{ng} / \mathrm{mL}$ and $0.014 \mathrm{ng} / \mathrm{mL}$, respectively showing a decline in the PFSA concentrations from 2004 to 2008.

In the Norwegian study of matched samples of serum and breast milk (sampled 2007-2008) the average median breast milk concentrations were $0.087 \mathrm{ng} / \mathrm{ml}$ for PFOS which corresponded to $1.4 \%$ of the corresponding serum concentrations for PFOS (Haug et al., 2011) i.e. transfer of PFOS to breast milk is lower than for PFCAs.

Elimination rates of PFOS in breast milk from nine Norwegian mothers in the Oslo area was studied by Thomsen and co-workers. The median concentration of PFOS was $0.11 \mathrm{ng} / \mathrm{ml}$. During lactation, PFOS cocentration in breast milk was reduced by $3.8 \%$ per month, and by $37 \%$ by year.

Sundström et al. measured the concentration of PFOS and PFHxS in pooled human milk samples obtained in Sweden between 1972 and 2008 (Sundstrom et al., 2011). PFOS and PFHxS demonstrated statistically significant increasing trends in pooled human milk samples from Stockholm over the period 1972-2000. PFOS and PFHxS showed a decline between 2001 and 2008 of approximately 13\% and $6 \%$ per year, respectively. In 2008, the measured concentrations of PFOS and PFHxS were $0.075 \mathrm{ng} / \mathrm{mL}$ and $0.014 \mathrm{ng} / \mathrm{mL}$, respectively. The study showed that the temporal trend in PFOS and PFHxS concentration in pooled human milk samples is similar to the trend in serum concentrations (Sundstrom et al., 2011).

For comparison, in the study of 19 breast milk samples from China (sampled 2004), PFHxS, PFOS were detected in all samples (So et al., 2006). Concentrations of PFOS ranged from 45 to $360 \mathrm{ng} / \mathrm{L}$ and up to 62 $\mathrm{ng} / \mathrm{L}$ for PFHxS.

The PFC concentrations in breast milk are far less than those reported in human blood or serum, however there might be a potential risk to infants because of the relatively high exposure per body weight compared to adults.

\section{Levels in amniotic fluids}

Only one unpublished and two published studies were found measuring PFSAs in human amniotic fluid (Jensen et al., 2012; Stein et al., 2012) (Bonefeld-Jørgensen, Unpublished data) (Table 12). Jensen et al. studied 300 randomly selected second-trimester amniotic fluid samples from a Danish pregnancy-screening biobank covering 1980 through 1996 (Jen- 
sen et al., 2012). Only PFOS was measured and the median concentration was 1,1 ng/ml (interquartile range (IQR): $0.66-1.60 \mathrm{ng} / \mathrm{mL}$ ). For each later gestational week of amniocentesis the PFOS was $9.4 \%$ higher (95CI: $3.3 \%, 15.9 \%$ ). No associations with maternal age or parity were found.

In the unpublished Danish study (Bonefeld-Jorgensen et al.) the concentrations of six PFSAs were measured in 54 amniotic fluid samples (1995-1999). The results showed that PFOS, PFOSA and PFHxS could be detected in $46.6 \%, 35.2 \%$ and $4.5 \%$ of the samples. The average PFOS level was $1.35+0.83 \mathrm{ng} / \mathrm{ml}$.

In the US study (sampled in 2005-2008), the concentrations of 2 PFSAs (PFHxS, PFOS) were measured in serum and amniotic fluid (second trimester) (Stein CR 2012). PFSAs in amniotic fluid was detected at concentrations approximately 10-20-fold lower than in maternal serum. PFOS was detected in 24 and PFHxS in 4 of the amniotic samples at median concentrations $0.4 \mathrm{ng} / \mathrm{ml}$. For the detected sulfonates strong correlations were seen between serum and amniotic fluid $(\rho=0.76-0.80)$.

PFOA appeared to be commonly detected in amniotic fluid if the serum concentration exceeded approximately $1.5 \mathrm{ng} / \mathrm{mL}$ whereas PFOS was rarely detected in amniotic fluid until the serum concentration was about $5.5 \mathrm{ng} / \mathrm{mL}$.

Amniotic fluid PFC concentrations are lower than in maternal blood (10-20 fold) and considerably lower than cord blood concentrations. The PFOS levels detected in the US study were lower than those reported from Denmark, probably due to the older Danish samples (1980-1990).

\subsubsection{Conclusions on human biomonitoring of PFSAs}

Blood levels of perfluorinated chemicals have been monitored in several Nordic countries including in the Arctic, and among PFSAs, usually PFOS and PFHxS are detected most frequently. PFHpS and PFBS have also been measured in the human blood, although not in all samples. PFDS were only observed above the LOQ in the Swedish study. Time trend analyses indicate decreasing tendency of PFOS and PFHxS since 2000, however, in Sweden an increasing tendency was observed for PFHxS from 1996 to 2010. Overall the Swedish studies show that the phase-out of PFOS-related chemicals has resulted in decreasing serum concentrations of PFOS in the blood serum of young Swedish women during the past decade. However, exposure to sulfonates with shorter carbon chains than PFOS (or their respective precursors), such as PFBS, is currently increasing. 
Correlations have been seen between PFOS and PFOA ${ }^{25}$ which indicate common sources for human exposures. In general, the blood level is higher for males than females.

High intake of fruit suggests lower blood level of PFOS and PFHpS, but seem to be caused by lower intake of fish and meat.

In summary, the studies found a correlation between the maternal and cord blood levels; and lower PFSA concentrations in cord serum than in maternal serum. PFOS and PFHxS were detected in cord blood and the studies showed that PFHxS was passed more easily through the placenta. PFCs with a short chain length seemed to pass the placenta more efficiently than PFCs with a longer chain length. PFCs with sulfonic acid as the active group seemed to pass more easily into the fetal circulation than PFCs with carboxylic acid as the active group.

Among PFSAs, only PFOS has been detected in breast milk in concentrations approximately $1-2 \%$ of the corresponding concentrations in serum. Only in the Swedish studies PFHxS was detected.

Few data on PFSAs (PFOS) in amniotic fluid is reported until now. Amniotic fluid PFC concentrations are considerably lower than maternal blood (10-20 fold) and also lower than cord blood concentrations. PFOS was more rarely detected in amniotic fluid until the serum concentration was about $5.5 \mathrm{ng} / \mathrm{mL}$; probably because PFOS appeared to be less soluble in amniotic fluid. For the detected sulfonates strong correlations were seen between serum and amniotic fluid.

\subsubsection{PFAL (Perfluoro aldehydes) in humans}

No biomonitoring data were found for exposure in humans.

\subsubsection{FTOH (fluorotelomer alcohols) in humans}

FTOHs are precursor compounds that are known to degrade to PFCAs. The measurement and human exposure to FTOHs has not yet been established but it has been shown that FTOHs (8:2-FTOH and 10:2-FTOH) are metabolized to PFCAs in vivo and in vitro studies (Dinglasan et al., 2004).

Therefore, PFCA can give som indication of the FTOH exposure. Below are some studies on PFCAs and FTOHs in air and associations to PFCA in serum are given.

25 The correlations were not limited to only PFOS and PFOA. 
Studies from Norway and Sweden showed elevated levels of some PFCAs indicators of the FTOH exposure, with carbon chain lengths from C4 to C11 in whole blood from ski technicians using fluorinated ski wax (Freberg et al., 2010; Nilsson et al., 2010a; Nilsson et al., 2010b). The median blood level of PFOA was $112 \mathrm{ng} / \mathrm{mL}$ in the Swedish study and 50 $\mathrm{ng} / \mathrm{ml}$ in the Norwegian study, which is $25-50$ times the concentration in the general population in these two countries. For the first time the PFTeDA was found in human serum (Freberg et al., 2010). PFNA was the second most abundant carboxylate in blood from wax technicians (Nilsson et al., 2001). The levels of the other PFCAs were also much higher than general population except for PFUnDA and PFTrDA (Nilsson et al., 2010a).

The PFC measurements of the ski wax working place in Sweden showed that the most dominating compound in the air samples was the 8:2 FTOH (range $=830-250,000 \mathrm{ng} / \mathrm{m} 3$ ), followed by PFHxA (range = $57-14,000 \mathrm{ng} / \mathrm{m} 3$ ) and PFOA (range $=80-4,900 \mathrm{ng} / \mathrm{m} 3$ ) (Nilsson et al., 2010a). The levels of the PFCAs found in the serum correlated well with those detected in air samples collected during ski waxing, supporting that inhalation is a major route of occupational exposure. The levels of PFSAs in ski waxes were comparable with those in the Norwegian general population. The authors of the Swedish study suggest that the internal exposure to PFOA was more likely indirect through biotransformation of 8:2 FTOH to PFOA and PFNA in humans (Nilsson et al., 2010a).

Another study aimed to investigate the role of indoor office air on exposure to PFCs among office workers ( $\mathrm{n}=31 ; 5 \mathrm{men}$ ) (Fraser et al., 2012). In the newly constructed building the air samples contained mainly FTOHs (8:2 FTOH $(9,920$ pg/m3); 10:2 FTOH (2,850 pg/m3); 6:2 FTOH $(1,320 \mathrm{pg} / \mathrm{m} 3))$ and MeFOSE $(289 \mathrm{pg} / \mathrm{m} 3)$ and to a lesser extent FOSAs. In the serum samples they detected PFOS $(11 \mathrm{ng} / \mathrm{mL})$, PFOA (3.7 $\mathrm{ng} / \mathrm{mL}$ ), PFNA (1.6 ng/mL), PFHxS (1.5 ng/mL), and PFDeA $(0.36$ $\mathrm{ng} / \mathrm{mL}$ ). PFUA, NMeFOSAA, N-EtFOSAA, and PFDoA were not detected in all samples and the concentrations were low. They reported a strong positive association between FTOH concentrations in office air and serum PFOA concentrations and weakly between FTOHs in office air and PFNA in serum. Evaluation: the authors suggested that inhalation of FTOH is an important exposure pathway to PFCAs. 


\subsubsection{FTS (fluorotelomer sulfonates) in humans}

No studies concerning biomonitoring for fluorotelomer sulfonates was found for the Nordic countries.

Exposure to legacy and current commercial fluorinated chemicals was investigated by analyzing fifty human sera samples collected in 2009 from the United States (Lee and Mabury, 2011).

The 8:2 FTS was the dominant congener observed in human sera ( $<\mathrm{LOD}(0.005 \mu \mathrm{g} / \mathrm{L})-0.231 \mu \mathrm{g} / \mathrm{L} ;>95 \%$ of the samples), followed by $6: 2$ FTS $(<\mathrm{LOD}(0.005 \mu \mathrm{g} / \mathrm{L})-0.047 \mu \mathrm{g} / \mathrm{L} ;>54 \%)$ and $4: 2$ FTS $(<\mathrm{LOD}$ $(0.005 \mu \mathrm{g} / \mathrm{L})-0.018 \mu \mathrm{g} / \mathrm{L} ;<20 \%)$. The observation of different perfluoroalkyl chain lengths of FTS in human sera here is consistent with exposure to fluorotelomer-based products. The sources of this contamination may include exposure to commercial products containing the FTS themselves, or to other fluorotelomerthiol-based products, such as FTMAPs.

\subsubsection{PAP/di-PAP (polyfluoroalkyl phosphate esters) in humans}

No studies concerning biomonitoring for polyfluoroalkyl phosphate esters (PAP/di-PAP) was found for the Nordic countries.

D'eon, JC et al. examined pooled human serum samples collected in 2004-2005 ( $\mathrm{n}=10)$ and $2008(\mathrm{n}=10)$ from the Midwestern US for the 4:2 through 10:2 PAP diesters (diPAPs) (D'Eon et al., 2009). The serum samples from 2004 and 2005 contained $4.5 \mu \mathrm{g} / \mathrm{L}$ total diPAPs, with the 6:2 diPAP dominating the congener profile at $1.9 \pm 0.4 \mu \mathrm{g} / \mathrm{L}$. As diPAPs have been shown to degrade to PFCAs in vivo, our observation of diPAPs in human sera may be a direct connection between the legacy of human PFCA contamination and PAPs applications.

In serum samples from 2009 lower diPAP concentrations $(0.035-0.136 \mu \mathrm{g} / \mathrm{L})$ for the more dominant congeners $(6: 2,6: 2 / 8: 2,8: 2)$ were detected (Lee and Mabury, 2011).

\subsubsection{Other fluorinated telomers in humans}

No biomonitoring data were found for exposure in humans. 


\subsubsection{Other fluorinated compounds of interest in humans}

Lee $\mathrm{H}$ et al. investigated the exposure to current commercial fluorinated chemicals by analyzing 50 human sera samples collected in 2009 from the United States for forty fluorinated analytes that included the one fluorotelomer mercaptoalkyl phosphate diester congener (FTMAP), perfluorophosphonates (PFPAs), and perfluorophosphinates (PFPiAs) (Lee and Mabury, 2011). The 6:2 FTMAP were not detected. PFPiAs were detected for the first time in human sera, with C6/C6 and C6/C8 PFPiAs as the dominant congeners, observed in $>50 \%$ of the samples. Unlike the PFCAs and PFSAs, there are no known PFPiA precursors in production. Therefore, the observation of PFPiAs in human sera gives evidence of human exposure to these chemicals.

No data for fluorotelomer mercaptoalkyl phosphate diester congener (FTMAP) was found for the Nordic countries.

\subsubsection{Conclusion on PFAL, FTOH, FTS, PAP/di-PAP and other PFC telomers in humans}

No data for PFAL (perfluoroaldehyde) in humans was found.

Data on FTOH in humans is not found since the measurement and human exposure and thus monitoring to FTOHs has not been established. However, it has been shown that FTOHs (8:2-FTOH and 10:2FTOH) are metabolized to PFCAs in in vivo and in vitro studies. Strong correlation between PFOA and FTOH are observed, and therefore PFOA serum levels can give an estimation of FTOH exposure and serum levels. Studies in Norway and Sweden showed elevated PFCAs with carbon chain length of C4-C11 in whole blood of occupational ski wax technicians with a level being up to 25-50 times that of the general population.

For office workers in a new building a strong correlation between air FTOH and PFOA serum concentration (more weakly for PFNA) was found.

No data on FTS (fluorotelomer sulfonates) for the Nordic countries was found. Whereas a US study reported that the 8:2 FTS was dominant in human sera, and that the sources may include exposure to commercial products containing FTS or FTMAPs (fluorotelomer mercaptoalkyl phosphate diester).

No data on PAP/di-PAP in humans has been reported for the Nordic countries. Whereas a US study (2004-2008) found 6:2 diPAP to be the dominating compound with decreasing levels observed in samples from 2009. Since diPAPs degrade to PFCA in vivo the diPAP serum level can also be indicative of PFCA exposure. 
A US study reported in 2009 data on human serum concentration of PFTMAP congeners. The C6/C6 and C6/C8 PFPiAs were the dominating congeners found in more than $>50 \%$ of the samples. Since there are no PFPiA precursors in production the data give evidence of human exposure to the chemicals.

\subsection{Suggested priority list of substances}

As a result of the findings concerning occurrence in the Nordic environment and in humans the following priority list of PFCs was agreed upon with KLIF/NORAP:

1. PFCAs, $\mathrm{C} 4$ and higher homologues with very low focus on $\mathrm{C} 8$.

2. FTOH, $\mathrm{C} 4$ and higher.

3. PFSA, C4 and higher with very low focus on C8.

4. FTS, 6.2 and 8:2 (mayby 10:2).

5. $\mathrm{diPAP} / \mathrm{monoPAP}$.

6. PFPE.

\subsection{Overall conclusion for the human biomonitoring data on PFCA, PFSA and other PFC telomers}

For both PFCAs and PFSAs human biomonitoring data for the Nordic countries during the period from 1992 to 2010 are found with most and newest data from Norway and Sweden, and fewest from Denmark. No human data were found for Iceland and Finland.

Overall, a decreasing tendency has been observed for PFOA and PFOS since 2002, whereas, in Sweden it was found that sulfonates with shorter carbon chains than e.g. PFOS or respective precursors is currently increasing.

Some Nordic studies $(n=4)$ also show that PFAAs can be transferred to the fetus (cord blood) and most efficiently for shorter chain length.

Although the longer carbon chains are known to be more biologically persistent than the shorter carbon chain compounds the daily exposure (including mixture) from several sources must be taken into consideration in risk assessment.

Only one Nordic study from Denmark on PFSAs and PFCAs in amniotic fluids has been published and another DK study has not yet been pub- 
lished. PFOS, PFHxS and PFOA were determined in a level being 10-20 folds below the serum level.

Five Nordic studies also show that the PFSAs and PFCAs can be transferred to human breast milk being in the concentration range of $1-2 \%$ and $3-4 \%$ of the corresponding serum concentration.

\subsection{Future work}

Thus Nordic studies are needed to follow up on the increase of sulfonates with shorter carbon chains and respective precursors in as well human/maternal blood and cord blood.

Further Nordic studies on different PFSA and PFCA congeners in amniotic fluids and breast milk are needed to explore the real pre-term and post term exposure of the fetus and newborn child.

Studies on the correlation between exposure to FTOH and PFCA at the serum level are needed to explore the general as well as occupational exposure to FTOHs.

Studies are needed for the Nordic countries on PFAL (perflouroaldehyde), FTS (fluorotelomer sulfonates), PAP/di-PAP and FTMAPs (fluorotelomer mercaptoalkyl phosphate diester) since no data are found. 


\title{
8. Human health effects and related animal toxicity of per- and polyfluorinated substances
}

\begin{abstract}
Most data found on human and animal toxicology and effects of PFCs are on PFOS and PFOA, probably because existing laboratory procedures in the past did not allow analyses of other PFCs that in general exist in lower concentrations and below detection limits; but also because PFOS and PFOA are the most abundant PFCs in the human matrix. Main epidemiological and medical surveillance studies have been conducted primarily in the United States on workers occupationally exposed to PFOS-based fluorochemicals (e.g. 3M) or populations exposed to PFOA-contaminated drinking water. These studies specifically examined PFOS or PFOA exposures and possible adverse outcomes such as mortality and cancer incidence studies. Further studies have been reported on potential endocrine effects, associations between primarily PFOS and/or PFOA serum concentrations and hematology, hormonal and clinical chemistry parameters. The results of the human studies are summarized in Table 13 and 14.
\end{abstract}

\subsection{PFCA (Perfluoroalkyl carboxylates)}

\subsubsection{PFBA (C4)}

\section{Animal experimental studies}

Several studies have been published on the potential biological responses of exposure to PFBA in experimental animals (Chang et al., 2008; Das et al., 2008; Permadi et al., 1992; Takagi et al., 1991) and in studies conducted in primary rat hepatocytes (Intrasuksri and Feller, 1991; Intrasuksri et al., 1998; Vanden Heuvel et al., 1991). In general, PFBA was effective in inducing peroxisome proliferation in these rodent studies but the in vivo potency was lower than for PFOA at same doses. In vitro, 
at the molecular level, PFBA were shown to activate both the human and mouse isoforms of peroxisome proliferator-activated receptor $\alpha$ (PPAR $\alpha$ ) in a transfection assay system in COS-1 cells (Wolf et al., 2008), but again with lower response compared to PFOA. Treatment of mice with PFBA during gestation did not result in any developmental effects as seen for PFOA (Das et al., 2008). This difference may be due to lower potency of PFBA to activate PPAR $\alpha$, or due to rapid elimination of PFBA (Chang et al., 2008).

A very recent study evaluated the toxicity of PFBA in male and female rats (28-day and 90-day) (Butenhoff et al., 2012). Effects in males included: increased liver weight, slight to minimal hepatocellular hypertrophy; decreased serum total cholesterol; and reduced serum thyroxin with no change in serum thyrotropin; no effects were seen in females. No effect on the endocrine system was found.

In summary, rodent exposures to PFBA induce peroxisome proliferation but at a lower level than PFOA. Studies suggested liver toxicity and some effect on the thyroid system. The reported studies suggest minimal developmental and endocrine effects from PFBA exposures.

\section{Human studies}

No data found.

\subsubsection{PFHXA (C6)}

\section{Animal experimental studies}

A 90-day repeated dose oral study in rats investigated the possible toxic effects of PFHxA at levels up to $200 \mathrm{mg} / \mathrm{kg} /$ day (functional observational battery and motor activity) (Chengelis et al., 2009). The observed changes included: lower body weight gains in the 10,50 and $200 \mathrm{mg} / \mathrm{kg} /$ day group males; lower red blood cell parameters; higher reticulocyte counts and lower globulin in the $200 \mathrm{mg} / \mathrm{kg} /$ day group of both males and females, higher liver enzymes in males at 50 and $200 \mathrm{mg} / \mathrm{kg} /$ day and lower cholesterol, calcium in males at $200 \mathrm{mg} / \mathrm{kg} /$ day. Based on liver histopathology and liver weight changes, the NOAEL for oral administration was 50 $\mathrm{mg} / \mathrm{kg} /$ day for males and $200 \mathrm{mg} / \mathrm{kg}$ /day for females. PFHxA was a poor peroxisomal inducer. No reproductive and developmental toxicity was reported for PFHxA (Loveless et al., 2009).

In summary, studies suggest that PFHxA exposures of rats affect body and liver weight, changes in liver and hematologic parameters, and that PFHxA is a poor peroxisomal inducer. 
Human studies

No data found

\subsubsection{PFNA (C9)}

\section{Animal experimental studies}

A 90-day rat PFNA feeding study with a 60-day recovery period suggested that the liver was the main target organ, with effects on serum clinical chemistry, higher liver weights, and evidence of induced peroxisome proliferation in both males and females. At the end of the recovery period most of the affected parameters had partially or completely returned to normal (Mertens et al., 2010).

PFNA has been shown to induce developmental toxicity and liver enlargement in mice when administered throughout the gestational period (Wolf et al., 2010). Using a PPAR knockout mouse model Wolf and coworkers showed that the effects of PFNA on survival rate and development of prenatally exposed mice was dependent on expression of PPAR (Wolf et al., 2010).

Immune toxic effects have also been observed for PFNA. Exposure of mice to PFNA for 14-days led to a decreased weight of the lymphoid organs, and the study suggested immune toxic effects on lymphoid organs and T cell (Fang et al., 2008). Cell apoptosis in rats can be caused by PFNA (Fang et al., 2010). Recently, PFNA was suggested to disrupt the hepatic glucose metabolism by increasing the levels of rat serum glucose and hepatic glycogen via altering the expression of the genes related to glucose metabolism and suppressing the hepatic insulin pathway (Fang et al., 2012).

In summary, studies suggest that PFNA exposures of rodents are related to liver toxicity including disrupting glucose metabolism; PPAR dependent effects on development and survival upon in utero exposure and PFNA induced immune toxicity. A proposal to classify PFNA as a reproductiv toxicant in the EU has been submitted by Sweden (ECHA, 2012a).

\section{Human studies: Occupational}

One occupational study of exposures to a PFNA surfactant blend was undertaken. The PFNA study examined liver enzymes and blood lipid levels (Mundt et al., 2007) in a cohort consisting of 630 employees at a U.S. polymer production facility using PFNA between 1989 and 2003. The exposure was assessed by work history and not by measuring the PFNA concentrations in the serum. Seven parameters of liver function (total cholesterol, Gamma glutamyl transpeptidase, transminases, alkaline phosphatase, bili- 
rubin, and triglycerides) were examined and they showed no significant effect. This study did not either find any effect on thyroid function as assessed by serum levels of TSH, T4, fT4 and T3 uptake.

The authors concluded that based on laboratory measures assessed over more than a decade; no adverse clinical effects were detected from occupational exposure to a PFNA blend.

\section{Human studies: general exposures (PFNA)}

- Lipid metabolism and cholesterol

In a cross-sectional study of general US population (NHANES data; 2003-2004) Nelson et al. investigated the relationship between exposure to PFNA (also PFOA, PFOS and PFHxS), and cholesterol levels, obesity and insulin resistance (Nelson et al., 2010). Total cholesterol and non-HDL were positively associated with PFNA levels. Another study from NHANES data (1999-2000 and 2003-2004) examined the relationship between PFCs and components of the metabolic syndrome in participants (Lin et al., 2009). They found that in adolescents, increased serum PFNA concentrations were associated with decreased blood insulin and clinical hyperglycaemia, increased serum HDL cholesterol, and was inversely associated with the prevalence of metabolic syndrome.

- Thyroid function

In a study of New York State anglers $(n=31)$, potential associations were investigated between serum concentrations of 5 measured carboxylates (PFDA, PFNA, PFHpA, PFOA, PFUnA) and levels of TSH and free T4 (Bloom et al., 2010). No statistically significant associations were found for PFNA (mean concentration $0.79 \mathrm{ng} / \mathrm{mL}$ (0.66-0.96)).

- Reproductive effects

A recent study evaluated the possible association between PFC exposure and biomarkers of male reproductive health in a larger population of almost 600 men from Greenland, Poland and Ukraine (Toft et al., 2012). PFNA (median: $1.2 \mathrm{ng} / \mathrm{mL}$ ) was not associated with sperm concentration, volume, total count or percent motile sperm. However, PFNA was associated with a non-significant lower proportion of normal sperm at higher exposure.

A study evaluated the association of female serum concentrations of different PFCs (PFOA, PFNA, PFDA) with time to pregnancy (TTP) in 222 Danish first-time pregnancy planners (prospective design) (Vestergaard et al., 2012). No clear association between PFNA (median concentration $0.45 \mathrm{ng} / \mathrm{mL}$ ) and TTP were observed. 
- Developmental effects

In a small Canadian study ( $\mathrm{n}=101$; sampling date 2004-2005) no association was found between birth weight and maternal PFNA levels (Monroy et al., 2008).

A US study examined the association between PFC exposure and ADHD among children (Hoffman et al., 2010). Data from this NHANES study ( $\mathrm{n}=571$; age 12-17; from 1999-2000 and 2003-2004) showed increased odds of ADHD disease with higher serum PFNA level (Odds ratio OR 1.32).

- Immune system

A study investigating childhood infection (immunoglobulin E) found no significant correlation between cord blood PFNA and serum total IgE or cord blood IgE in 244 2-year old Taiwanese children (Wang et al., 2011). In summary, there are very few data on the suspected health effects of PFNA and further studies are needed to explore the adverse effects of PFNA in human.

\subsubsection{PFDA (or PFDeA) (C10)}

\section{Animal experimental studies}

Animal studies have identified PFDA as toxic to the liver. Administration of single gavage doses of $\geq 20 \mathrm{mg} / \mathrm{kg}$ PFDA to female C57BL/6N mice resulted in significant and dose-related increases in relative liver weight, assessed 30 days after dosing (Harris and Birnbaum, 1989; Harris et al., 1989). Significant elevations in liver weight (69\%) were seen in mice 2 days after treatment with $40 \mathrm{mg} / \mathrm{kg}$ PFDA (Brewster and Birnbaum, 1989), which also significantly increased hepatic acyl-CoA oxidase activity and hepatic lipids. Kawashima et al. (Kawashima et al., 1995) compared the effects of lower dietary doses of PFDA (1.2-9.5 mg/kg/day) and PFOA (2.4-38 mg/kg/day) on hepatic effects in male rats in a 7-day dietary study. PFDA was considerably more potent than PFOA in reducing body weight gain, food consumption, causing hepatomegaly, and inducing biochemical markers of peroxisome proliferation.

Other reported animal effects of PFDA: increasing serum cholesterol in male rats exposed to $10 \mathrm{mg} / \mathrm{kg} /$ day (Shi et al., 2007); 2-and 4-fold increases in serum T3 and T4, respectively, 30 days after a single dose of $80 \mathrm{mg} / \mathrm{kg}$ PFDA to female C57BL/6N mice (Harris et al., 1989); reduction of body weight (33\%) in male C57BL/6 mice $(78 \mathrm{mg} / \mathrm{kg} /$ day) (Permadi et al., 1992); potent inducer of the estrogen-responsive biomarker protein vitellogenin (Vtg) in juvenile rainbow trout (Benninghoff et al., 
2011), decreased testicular androgen production (plasma testosterone and dihydrotestosterone levels) in male rats (Bookstaff et al., 1990).

In summary, the animal studies have demonstrated that the liver is the primary target for PFDA toxicity including inducement of the peroxisome proliferation and acyl-CoA oxidase activity; the PFDA had a higher toxic potency than PFOA. Furthermore, effects on the thyroid and testicular androgen level were observed in mice and rats, respectively. A proposal to classify PFNA as a reproductiv toxicant in the EU has been submitted by Sweden ((ECHA), 2012b).

\section{Human studies: general exposures (PFDA)}

- Thyroid function

In the study of New York State anglers $(\mathrm{n}=31)$, PFDA was above the LOD for $65 \%$ of the samples (mean $0.21 \mathrm{ng} / \mathrm{ml}(0.18-0.26)$ ). No associations were found between serum concentrations of PFDA and levels of TSH and free T4 (Bloom et al., 2010). However the authors suggested that there was a possibility of weak positive associations between FT4 and PFDA by use of a larger sample size (Bloom et al., 2010).

- Reproductive effects

A study evaluated the association of female serum concentrations of different PFCs (PFDA, PFOA, PFNA) with time to pregnancy (TTP) in 222 Danish first-time pregnancy planners (prospective design) (Vestergaard et al., 2012). No clear association between PFDA (median: $0.10 \mathrm{ng} / \mathrm{mL}$ ) and TTP was observed.

- Developmental outcome

No associations were found between PFDA exposure and birth weight in a Canadian study (Monroy et al., 2008) or between PFDA exposure and ADHD among children from US NHANES study (Hoffman et al., 2010).

- Immune system A study investigating childhood infection (immunoglobulin E) found no significant correlation between cord blood PFDA and serum total IgE or cord blood IgE in 244 2-year old Taiwanese children (Wang et al., 2011). In summary, there are very few data on the suspected health effects of PFDA and further studies are needed to explore the adverse effects of PFDAs in human. 


\subsubsection{PFUnDA/ PFUnA (C11)}

\section{Animal experimental studies}

In vitro, PFUnA could at low level activate the mouse PPAR $\alpha$ in transiently transfected COS-1 cells (Wolf et al., 2012).

No further animal experimental toxicity data were found.

\section{Human studies (PFUnA)}

In the study of New York State anglers $(\mathrm{n}=31$; PFOA mean conc. 1.33 $\mathrm{ng} / \mathrm{mL}$ ), no associations were found between serum concentrations of among others PFUnA and levels of TSH and free T4 (Bloom et al., 2010). However the authors suggested that there was a possibility of weak positive associations between FT4 and PFUnA by use of a larger sample size (Bloom et al., 2010).

In two Korean studies, no correlations were found between PFUnA exposure and TSH and T4 levels (Ji et al., 2012) in the general population and neither between PFUnA in maternal blood serum and T3/T4/TSH of fetal cord blood serum (Kim et al., 2011).

Very few studies have measured and evaluated the PFUnA in the human studies, and they found no correlations to the assessed health effects.

\subsubsection{PFDoA or PFDoDA (C12)}

\section{Animal experimental studies}

Treatment of male rats with PFDoA by gavage for 14 days significantly induced an increase in total serum cholesterol $(10 \mathrm{mg} / \mathrm{kg} /$ day $)$ and reduction in body weight $(5 \mathrm{mg} / \mathrm{kg} /$ day). PFDoA exposure at $5 \mathrm{mg} / \mathrm{kg} /$ day or $10 \mathrm{mg} / \mathrm{kg} /$ day resulted in testis cell (Leydig and Sertoli) apoptosis and a decline in serum estradiol and testosterone levels (Shi et al., 2007). Another study showed that 110 days of PFDoA exposure led to significantly decreased testosterone and expressional changes of testicular steroidogenic genes in rats (Shi et al., 2009). The authors suggested that testosterone decline may be involved in the pathway of cholesterol transportation and steroidogenesis, and that these pathways were disrupted in testes following PFDoA exposure (Shi et al., 2007).

In summary, rodent studies suggest that PFDoA exposures decreases body weight, causes liver and testes toxicity, including changes in an array of parameters such as serum cholesterol and testes hormone level. 


\section{Human studies}

In the few studies that measured the PFDoA, there was not found any association with thyroid hormone, semen quality or lipid metabolism (Joensen et al., 2009; Kim et al., 2011) (Halldorsson et al., 2012). In some of these studies PFDoA was found in few samples above LOD.

\subsubsection{PFTrDA (C13)}

\section{Animal experimental studies}

No data on the toxicity of PFTrDA were found.

\section{Human studies}

In the Korean studies, investigating the exposure levels of 13 PFCs, PFTrDA were detected in $>90 \%$ of maternal serum samples $(0.39 \mathrm{ng} / \mathrm{mL}$ (0.27-0.57). One study observed that only PFTrDA concentrations were positively correlated with TSH levels, but negatively with total T4 concentrations among the female population (Ji et al., 2012). A positive association was detected between PFTrDA in maternal blood serum and T3 and T4 of fetal cord blood serum and between PFOA and cord serum TSH (Kim et al., 2011).

In another study where PFTrDA was measured, PFTrDA was under LOD in many of the samples (Joensen, 2009).

\subsubsection{PFOA (C8)}

\section{Animal experimental studies}

There is a considerable amount of animal data on the health effects of PFOA, which has been reviewed (Lau et al., 2007). The relevance of animal data for humans is controversial because of a much shorter half-life in rodents (measured in days) and the possible dependence of some animal toxicity on a peroxisome proliferation mechanism that is likely to be less important in humans (Steenland et al., 2010a).

In its draft risk assessment, the U.S. EPA (2005) concluded that evidence was suggestive that PFOA is carcinogenic in humans. In its review of that risk assessment, three of the four members of the EPA scientific advisory board concluded more strongly that PFOA was "likely to be carcinogenic in humans" (U.S. EPA 2006). There has been submitted a proposal for harmonised classification and labelling of PFOA/APFO in EU by Norway. In December 2011 the Risk Assessment Committee of the ECHA came to the conclusion that classification according to regulation EC No. 1272/2008 for PFOA is Repr. 1B and STOT RE 1 (ECHA, 2011). 


\section{- Tumor induction}

PFOA was not mutagenic or genotoxic in the classic battery of test for genotoxicity and mutations detected by the Ames test and structural chromosome damage (Fernandez Freire et al., 2008). However, in human HepG2 cells this compound exerted genotoxic effects as a consequence of oxidative stress (Yao and Zhong, 2005). In rodents dietary intake of PFOA induced tumors of the testicles, liver, and pancreas (Biegel et al., 2001; Sibinski, 1987). A 2-year study in rats reported a statistically significant increase in mammary fibroadenomas and Leydig cell adenomas suggesting impact of PFOA on reproductive tissues (Sibinski, 1987). In 2007 White and coworkers reported gestational exposure to PFOA in mice was associated with altered mammary gland development in dams and female offspring (White et al., 2007).

- Hepatotoxicity

The liver is the primary target organ by the exposure of animals to PFOA (and PFOS). PFOA has been shown to induce peroxisome (microbodies) proliferation in mouse and rat liver, and causes hepatomegaly. This proliferation has been shown to alter lipids, liver enzymes, and liver size (Kennedy et al., 2004; Lau et al., 2007; Takagi et al., 1992). Peroxisome induced proliferation and the resulting activation of a nuclear receptor peroxisome proliferator-activated receptor $\alpha$ (PPAR $\alpha$ ) have also been proposed as a mechanism for tumor induction and for the immune and hormonal changes seen in rodents (Lau et al., 2007). Other proposed mechanism for tumor promotion is by inhibition of gap-junctional intercellular communication (GJIC)(Upham et al., 2009). GJIC plays a vital role in maintaining tissue homeostasis, and disruption of gap junction function can lead to diseased states such as tumorigenesis. PFOA was shown to decrease GJIC activity in the liver of rats treated for both acute and long-term dosing (Upham et al., 2009).

- Developmental toxicity The developmental toxicity of PFOA (and PFOS) has been examined in rats and mice (Butenhoff et al., 2004; Hinderliter et al., 2005; Lau et al., 2006). Generally, the developmental toxicity induced by exposure to PFOA throughout gestation included increased neonatal mortality, reduced postnatal survival, delayed eye opening, growth deficits, and sex-specific alterations in pubertal maturation (Lau et al., 2006). A cross-foster study indicated that the postnatal effects on survival, eye opening, and weight gain were a consequence of gestational exposure and that exposure via lactation was not a major 
factor (Wolf et al., 2007). Using a PPAR $\alpha$ - knockout mouse model it was shown that PFOA developmental toxicity was dependent on expression of PPAR $\alpha$ (Abbott et al., 2007).

- Immunotoxicity In rodents PFOA decreases the B-cell and T-cell immune responses and results in atrophy of the spleen and thymus (Yang et al., 2002), causes hepatomegaly (Takagi et al., 1992), and decreases levels of cholesterol (Kennedy et al., 2004).

- Endocrine disruption

Several experimental studies have reported that PFCs impair thyroid hormone homeostasis. Effects of PFOA on thyroid hormones are not as well characterized as those of PFOS. Depression of serum triiodothyronine (T3) and/or thyroxine (T4) in PFOA exposed rats and monkeys has been reported (Butenhoff et al., 2002; Lau et al., 2007), but without an expected corresponding elevation of thyroidstimulating hormone (TSH) through feedback stimulation of the hypothalamic-pituitary-thyroid axis. Increases in estradiol and decreases in testosterone with PFOA exposure have also been observed in rodents (Lau et al., 2007). In addition to thyroid hormone disruption, changes in sex steroid hormone biosynthesis have been reported. Administration of PFOA to adult male rats for 14 days led to a decrease in serum and testicular testosterone and an increase in serum estradiol levels (Biegel et al., 1995), which was suggested to be associated with aromatase induction in the liver.

- Neurotoxicity Not much data was found for neurotoxicity of PFCAs including PFOA. Neonatal exposure of mice to PFOA (and PFOS) affected the proteins involved in neurogenesis and synaptogenesis in the developing mouse brain, which were accompanied by neurobehavioral defects in adulthood (Johansson et al., 2008). Also in an avian model PFOA was reported to be a developmental neurotoxicant (Pinkas et al., 2010). In summary, PFOA exposures in rodent indicate carcinogenic responses in liver, pancreas, testes and mammary glands. Furthermore, PFOA exposure in animals affect the development and reproduction, thyroid and immune system negatively; induces peroxisome proliferation believed to be a mechanism behind tumor initiation and effect on immune- and hormone systems.

\section{Human studies: Occupational exposures}

The first retrospective cohort study on mortality of employees of the PFOA-producing factory (3M) demonstrated a significant association between prostate cancer mortality and employment duration (Gilliland and 
Mandel, 1993). In an updated study the previously found association between prostate cancer and time of employment could not be confirmed.

However, in a later study of exposed workers in the $3 \mathrm{M}$ factory in Minnesota, ammonium perfluorooctanoate (APFO) exposure was presumably associated with prostate cancer, cerebral vascular disease, and diabetes mellitus, but not with liver, pancreas, or testicular cancer (Lundin et al., 2009).

Based on the occupational exposures there were reported no significant associations between serum PFOA and reproductive hormones in men (Olsen et al., 1998). In the study of Olsen and Zobel (2007) PFOA was not statistically significantly associated with total cholesterol or low-density lipoproteins. High-density lipoprotein (HDL) and free T4 were negatively associated with the PFOA level, whereas triglycerides and T3 tended to be positively associated (Olsen and Zobel, 2007). Several studies have investigated the relation to liver toxicity. The liver enzymes, transaminase levels, were positively associated with PFOA serum concentrations in some studies (Olsen and Zobel, 2007) but not in others (Sakr et al., 2007), indicating controversial hepatotoxic effects.

\section{Human studies: Communities with high exposed population}

In the United States two communities in Minnesota and West Virginia/Ohio have been exposed via water contamination coming from adjacent industrial plants (water mean levels for PFOA: $15 \mathrm{ng} / \mathrm{ml}$ in Minnesota and $82 \mathrm{ng} / \mathrm{ml}$ in Ohio in 2005, respectively). Several studies describe the health effects of the PFOA in the "C8 Health Project" (http://publichealth.hsc.wvu.edu/c8/) which has data on 69,030 persons, and provide an opportunity to examine a very large population with high exposure median $(26.6 \mathrm{ng} / \mathrm{mL}$ of serum vs. $4 \mathrm{ng} / \mathrm{mL}$ in the general U.S. population). The results are summarized in Table 13.

The following associations have been seen for PFOA in the "C8 Health Project": increased total cholesterol and low-density lipoprotein in children and adolescents (Frisbee et al., 2010); increased blood lipid levels in relation to elevated PFOA (and PFOS) concentrations in the blood (Steenland et al., 2009); no associations to HDL cholesterol; positive associations with serum and liver enzymes (transaminase; a marker of hepatocellular damage) indicating hepatotoxic effect in humans (Gallo et al., 2012); positive association to serum uric acid (Steenland et al., 2010); no association between PFOA and TSH ( $\mathrm{n}=371)$ (Emmett et al., 2006); significant positive elevation in serum $\mathrm{T} 4$ and a significant reduction in T3 uptake in adults (Knox et al., 2011a); no associations with preterm birth and fetal growth restrictions (Savitz et al., 2012), positive association with hypothyroidism in children (Odds ratio (OR): 1.54; $95 \%$ 
confidence interval (CI): 1.00, 2.37) (Lopez-Espinosa et al., 2012); associations with Attention Deficit Hyperactivity Disorder (ADHD) in children 5-18 years of age, with a small increase in prevalence for the second quartile of exposure and a decrease for the highest versus lowest quartile (Stein and Savitz, 2011); more likely to have experienced menopause among perimenopausal women with higher level of PFOA (and PFOS), suggesting endocrine disrupting effects (Knox et al., 2011b); association with lower serum concentrations of IgA and IgE (for IgE in females only) (C8 Science Panel, 2009).

The C8 Science Panel has concluded its work in determining whether there is a probable link between exposure to $\mathrm{C} 8$ (PFOA) and a range of human diseases (http://www.c8sciencepanel.org/prob_link.html). The Science Panel did find a Probable Link between exposure to C8 and medically-diagnosed high cholesterol, and thyroid disease, testicular cancer and kidney cancer, and pregnancy-induced hypertension (elevated blood pressure in pregnancy).

The Science Panel found no Probable Link between C8 and the following diseases: Parkinson's disease, non-malignant liver disease, nonmalignant kidney disease, osteoarthritis, coronary artery disease or high blood pressure, adult onset diabetes, chronic obstructive pulmonary disease, asthma, childhood and adult infections such as influenza, neurodevelopmental disorders in children, stroke, and five other autoimmune diseases (lupus, rheumatoid arthritis, Type 1 (juvenile) diabetes, Type II (adult-onset) diabetes, Crohn's disease, and multiple sclerosis, risk of pregnancy loss, either miscarriage or stillbirth, preterm or low birth weight infants, measures of prematurity.

In summary, the Community high exposed population studies support the occcupational studies on hepatoxic and thyroid toxic effects of PFOA. In addition, association with ADHD in children, endocrine and immunotoxic effects and testis and kidney cancers was reported.

\section{Human studies: General population}

Few epidemiological studies exist with data from the general population. Below are summarized the adverse health effects observed for PFCAs (Table 14).

\section{- Lipid metabolism and cholesterol}

The discovery that PFCs bind to the PPARs (nuclear receptors that play a key role in lipid metabolism) have raised the concern that the PFCs may disrupt lipid and weight regulation. The occupational studies have suggested a positive association between PFOA and levels of cholesterol in humans. 
In a cross-sectional study of general US population (NHANES data; 2003-2004) Nelson et al. investigated the relationship between exposure to PFOA and PFNA (and also PFOS and PFHxS), and cholesterol levels, obesity and insulin resistance (Nelson et al., 2010). Total cholesterol and non-HDL was positively associated with PFOA (and PFNA and PFOS) level. The median serum concentration in NHANES was $4 \mathrm{ng} / \mathrm{mL}$.

Another study from NHANES data (1999-2000 and 2003-2004) examined the relationship between PFCs and components of the metabolic syndrome in participants (Lin et al., 2009). They found no correlation to PFOA, but in adolescents, increased serum PFNA concentrations were associated with decreased blood insulin and clinical hyperglycaemia, increased serum HDL cholesterol, and was inversely associated with the prevalence of metabolic syndrome. In a Danish study mothers who were overweight or obese before pregnancy had higher plasma levels of PFOA (and PFOS) (Fei et al., 2007), suggesting a relation between BMI and PFOA levels. In addition, a recent prospective study of pregnant women and their children 20 years later $(n=665$; maternal serum from 1988-1989) showed that in utero exposure to PFOA was positively associated with the prevalence of overweight and a high waist circumference at 20 years in female but not male offspring (Halldorsson et al., 2012). In summary, the present studies on human exposure to PFCAs suggest some liver toxicity with changes in parameters involved in metabolic syndrome such as lipids, cholesterol levels and non-HDL, with the risk of obesity and insulin resistance.

- Cardiovascular diseases

Melzer et al. found no trend in self-reported history of heart disease in adults from the NHANES, after dividing PFOA serum levels into quartiles (Melzer et al., 2010). However, in a recent study from NHANES, higher serum PFOA levels were positively associated with self-reported cardiovascular diseases including coronary heart disease and stroke, and objective peripheral arterial disease (Shankar et al., 2012).

- Cancer

A follow-up study of the general population in Denmark $(55,053$ Danish adults; 50-65 years of age; 1993-2006) found no clear differences in incidence rate ratios for bladder and liver cancers in relation to plasma concentrations of PFOA; although a modest positive associations were reported for prostate and pancreas cancers (Eriksen et al., 2009). A recent case-control study of Greenlandic Inuit (31 cases, 115 controls; collected in 2000-2003) 
showed a significant association between serum PFOA (sum of PFCs) and risk of breast cancer (Bonefeld-Jorgensen et al., 2011).

In summary, PFCAs might be risk factors in prostate, pancreas and breast cancers, but further studies are needed.

- Thyroid function

In a study of New York State anglers $(\mathrm{n}=31$; PFOA mean conc. $=1.33$ $\mathrm{ng} / \mathrm{mL}$ ), potential associations were investigated between serum concentrations of 5 measured carboxylates (PFDA, PFNA, PFHpA, PFOA, PFUnA) and levels of TSH and free T4 (Bloom et al., 2010). No statistically significant associations were found for any of the PFCs or the sum of them. A recent cross-sectional analysis of self-reported thyroid disease in the NHANES ( $\mathrm{n}=3,974$ adults) reported a significant association for PFOA and thyroid disease in general US population and particularly in females (Melzer et al., 2010). More women with blood concentrations of $\geq 5.7 \mathrm{ng} \mathrm{PFOA} / \mathrm{mL}$ were found to have currently treated thyroid disease compared with women having $\leq 4.0 \mathrm{ng} / \mathrm{mL}$ of blood levels.

In a Korean study, investigating the exposure levels of 13 PFCs, they observed no association to PFOA, but only PFTrDA concentrations was positively correlated with TSH level, but negatively with total T4 concentration among the female population (Ji et al., 2012). In summary, possible association for PFOA and thyroid diseases is suggested. Further studies are needed before any conclusion can be taken.

- Reproductive effects

A Danish study $(\mathrm{n}=105)$ showed an association between PFOA (and PFOS) exposure and the proportion of morphologically normal sperm cells (Joensen et al., 2009). However, an American study evaluated the semen quality among 256 infertility patients in relation to PFOS and PFOA in serum and semen and found no association between PFOS or PFOA levels and sperm concentration, volume or motility (Raymer et al., 2012).

A recent study evaluated the possible association between PFC exposure and biomarkers of male reproductive health in a larger population of almost 600 men from Greenland, Poland and Ukraine (Toft et al., 2012). Sperm concentration, total sperm count and semen volume was not consistently associated with PFOA.

Couple fecundity and time to pregnancy (TTP) were evaluated in the Danish National Birth Cohort study $(\mathrm{n}=1,240)$ (Fei et al., 2009). The evaluation of TTP showed increased risk of irregular menstrual cycles in the upper three quartiles of PFOA relative to the lowest quartile and an increase in mean PFOA with longer TTP. The odds of 
infertility ( $\geq 12$ months without conception) were elevated in the upper three quartiles of PFOA. Similar patterns were reported for PFOS (Fei et al., 2009). Among Norwegian pregnant women having given birth before, increased odds of sub-fecundity were associated with high PFOA and PFOS (Whitworth et al., 2012b). Among nulliparous women, higher PFC plasma level was associated with a decreased odd of subfecundity (Whitworth et al., 2012b). Another study evaluated the association of female serum concentrations of eight different PFCs (PFOS, PFOA, PFHxS, PFNA, PFDA, MeFOSAA, EtFOSAA and FOSA) with TTP in 222 Danish firsttime pregnancy planners (prospective design) (Vestergaard et al., 2012). Inconsistently with earlier observations no clear association between any of the measured PFCs and TTP were observed, which might be related to study design and the parity status of the women. In summary, controversial data on PFCA effects on human semen quality is reported, further studies must evaluate whether the compounds such as PFOA or PFNA affect the sperm output. Some studies suggest that PFCAs (PFOA, PFOS) affect the time to pregnancy and fecundity of females.

- Developmental outcome

The most extensive set of studies has examined foetal growth, birth weight, duration of gestation, and related indices of in utero development (Apelberg et al., 2007; Fei et al., 2007, 2008; Hamm et al., 2010; Hoffman et al., 2010; Monroy et al., 2008; So et al., 2006; Washino et al., 2009; Maisonet et al., 2012). In the Danish National Birth Cohort study (DNBC) ( $\mathrm{n}=1,400$ mother/child), Fei et al. investigated the possible correlations between the concentration of PFOA (and PFOS) in the maternal blood during the first and second trimesters of pregnancy and the birth weight and risk of premature birth (Fei et al., 2007). Only PFOA levels were inversely associated with birth weight. Gestational length was unaffected by PFOA concentrations. A statistically non-significant inverse association was also observed between PFOA and head circumference, and a positive association with newborn ponderal index (like BMI for babies) (Fei et al., 2008). Also another study from the US $(n=293)$ reported levels of cord blood PFOA (and PFOS) to be inversely associated with birth weight, new-born head circumference, crown-heel length, and ponderal index (Apelberg et al., 2007).

Data from a subgroup of the Norwegian Mother and Child Cohort study ( $\mathrm{n}=901$ women; 2003-2004) showed lower birth weight among infants born to mothers in the highest quartiles of PFOA (and PFOS) 
compared to lowest quartiles (Whitworth et al., 2012a).

Also a British study found that girls born to mothers with maternal serum concentrations of PFOA in the upper tertile weighed less $130 \mathrm{~g}$ (95\%CI: $-237,-30)$ ) at birth compared with girls born to mothers with serum concentrations in the lower tertile (Maisonet et al., 2012).

Contrary to the above findings, 2 smaller Canadian studies found no evidence of decreased birth weight and maternal PFOA levels (Monroy et al., 2008; Hamm et al., 2010).

In summary, in general Nordic and British studies suggest that PFOA affects the foetal growth negatively, whereas smaller Canadian studies did not see this effect.

Developmental milestones of children were examined in the substudy of the Danish National Birth Cohort $(n=1,400)$, where early pregnancy plasma PFOA levels were unrelated to motor or mental development through 18 months of age (Fei et al., 2008). Beside the study from "C8 Health project" in USA (Stein and Savitz, 2011), another study did examine the association between PFC exposure and ADHD among children (Hoffman et al., 2010). Data from this NHANES study from 1999-2000 and 2003-2004 ( $\mathrm{n}=571$; age 1217) showed increased odds of ADHD disease with higher serum PFOA and PFNA levels (OR1.12 and 1.32, respectively).

Further studies are needed to explore the suspected effects of PFCAs on CNS and child development including behavior and ADHD.

- Immune system

There are few epidemiological studies on the effects of PFOA on immune function related to infectious disease. A Danish study examined the prenatal exposure to PFOA (and PFOS) and the association with the infectious diseases in children (Fei et al., 2010) in the Danish National Birth Cohort. Hospitalizations for infection of the children were not associated with prenatal exposure to PFOA and PFOS. The authors concluded that their data did not support the hypothesis that prenatal PFOS and PFOA exposures decrease resistance to childhood infections.

Wang et al. (2011) found that cord blood PFOA was positively associated with cord blood serum IgE in 2-year old Taiwanese boys, whereas Okada et al. (2012) found that maternal serum PFOA was negatively associated with cord blood IgE in newborn Japanese girls However, in the latter study no relationship was found between maternal PFOA levels and infant allergies and infectious diseases at age in 18 months (Okada et al., 2012).

Recently, another prospective study of birth cohort from the National 
Hospital in the Faroe Islands $(n=656)$ reported a negative correlation with antibody response to tetanus and diphtheria booster immunizations at age 5 years with increasing serum PFOA (and PFOS) (Grandjean et al., 2012). The conclusion was that elevated exposures to PFAAs were associated with reduced immune response to vaccination.

Further studies are needed to reject or document the few studies on the suspected effect on the human immune system.

\subsubsection{Summary findings for PFCAs}

\section{Overall summary of animal toxicity upon PFCA exposure}

PFBA (C4) exposures in rodents induce peroxisome proliferation but at a lower level than PFOA. Study suggested liver toxicity and some effects on the thyroid system. The reported studies suggest minimal developmental and endocrine effects for PFBA exposures.

PFHxA (C6) exposures in rats affect body and liver weight, changes in liver and hematologic parameters, and PFHxA is a poor peroxisomal inducer.

PFNA (C9) exposures in rodents are related to liver toxicity including glucose metabolism; immune toxicity, and PPAR dependent effects on development and survival upon in utero exposure in knock out mouse. A proposal to classify PFNA as a reproductiv toxicant in the EU has been submitted by Sweden

PFOA (C8) exposure in rodent causes carcinogenic responses in liver, pancreas, testes and mammary glands. Furthermore, PFOA exposure in animals affect the development and reproduction, thyroid and immune system negatively; induces peroxisome proliferation believed to be a mechanism behind tumor initiation and effect on immune- and hormone systems.

PFDA (C10) exposures in animal studies have demonstrated that the liver is the primary target including inducement of the peroxisome proliferation and acyl-CoA oxidase activity; the PFDA had a higher toxic potency than PFOA. Furthermore, effects on the thyroid and testicular androgen level were observed in mice and rats, respectively. A proposal to classify PFDA as a reproductiv toxicant in the EU has been submitted by Sweden.

No animal experimental toxicity data were found for PFUnA (C11), however in vitro, at a low level PFUnA (C11) activated the mouse PPAR $\alpha$ in transiently transfected COS-1 cells. 
PFDoA (C12) exposures in rodents decrease the body weight, causes liver and testes toxicity, including changes in an array of parameters such as serum cholesterol and testes hormone level.

\section{Overall summary for human health effects upon PFCA exposure}

Data on occupational exposures to PFOA (C8) are controversial but suggest possible relation to the risk of prostate cancer, cerebral vascular disease, and diabetes mellitus, but not liver, pancreas, or testicular cancer. Moreover, there might be effects on steroid and thyroid hormone levels, whereas the relation to liver toxicity is controversial. Based on laboratory measurements assessed over more than one decade; no adverse clinical effects were detected from occupational exposure to PFNA (C9).

Data on community high-exposed population studies support the occcupational studies on hepatoxic and thyroid toxic effects of PFOA. In addition, association with ADHD in children, endocrine and immunotoxic effects, testis and kidney cancers and pregnancy-induced hypertension was reported.

For the general population the present studies on human exposure to PFCAs suggest some liver toxicity with changes in parameters involved in metabolic syndrome such as lipids, cholesterol levels and non-HDL, with the risk of obesity and insulin resistance.

Higher serum PFOA levels were found positively associated with selfreported cardiovascular diseases including coronary heart disease and stroke, and objective peripheral arterial disease. Moreover, PFCAs might be risk factors in prostate, pancreas and breast cancers, but further studies are needed.

Although further studies are needed, a possible association between PFOA and PFTrDA and thyroid diseases as well as weak positive relations between thyroid effects and FT4, PFDA and PFUnDA was suggested.

Controversial data on reproductive factors have been reported such as the effect of PFCA (PFOA, PFNA) on human semen quality and sperm output and need further studies. Some studies suggest that PFCAs (PFOA) affect the time to pregnancy and fecundity of females.

Some Nordic and British studies suggest that PFOA affects the foetal growth negatively, whereas some Canadian studies did not see this effect. Moreover, one Danish study on developmental milestones up to 18 month did not find any association to plasma PFOA levels; whereas one US study found an increased odds of ADHD disease with higher serum PFOA and PFNA levels at age 12 to 17. Further studies 
are needed to explore the suspected effects of PFCAs on CNS and child development including behavior and ADHD.

Further studies are needed to reject or document the few studies on the suspected effect on the human immune system.

\subsection{PFSA (Perfluoroalkyl sulfonates)}

The epidemiological studies of general population are summarized in Table 14.

\subsubsection{PFBS (C4)}

\section{Animal experimental studies}

PFBS (K+PFBS) was assessed for developmental and reproductive effects in a two- generational rat study (Sprague Dawley) (Lieder et al., $2009 b$ ). The study showed that maternal exposure to PFBS did not adversely affect the reproductive function in Sprague Dawley rats at doses as high as $1,000 \mathrm{mg} / \mathrm{kg} /$ day or developmental outcomes at doses as high as $300 \mathrm{mg} / \mathrm{kg} /$ day. In both the parental and F1-generation male, there were increased liver weight and histological changes (increased cell proliferation) in kidneys in the 300 and $1,000 \mathrm{mg} / \mathrm{kg} /$ day dose group rats. Similar kidney effects were reported in the 90-day study (Lieder $e t$ al., 2009a), and the authors discussed in that article that these changes likely were due to high concentrations of PFBS, a strong surface active compound, passing through the kidney, as urine is the major excretory route for PFBS (Olsen et al., 2009). In the 90-day study other observed effects included decreased red blood cell count, hemoglobin, and hematocrit at 200 and $600 \mathrm{mg} / \mathrm{kg}$ (Lieder et al., 2009a).

In summary, mild effects at the liver, kidney and blood parameters have been observed in rat studies upon exposure to PFBS (C4) at relatively high doses.

\section{Human studies (PFBS)}

No data found. 


\subsubsection{PFHxS (C6)}

\section{Animal experimental studies}

Only few published studies were found regarding potential toxicological properties of PFHxS in experimental animals.

A reproductive and developmental toxicity study of PFHxS was conducted in rats by York (York et al., 2010) (Butenhoff et al., 2009). In parental males there was a significant reduction in cholesterol already at doses $0.3 \mathrm{mg} / \mathrm{kg} /$ day, and hepatotoxicity at doses $3 \mathrm{mg} / \mathrm{kg} /$ day. No treatment-related effect was reported on the fertility and reproductive outcomes or on viability and growth of the offspring at doses as high as $10 \mathrm{mg} / \mathrm{kg} /$ day. A NOAEL of $10 \mathrm{mg} / \mathrm{kg} /$ day was therefore estimated for the developmental effects of PFHxS.

\section{Human studies (PFHxS)}

- Thyroid function

Among Koreans, PFHxS were not correlated to total T4 or TSH levels (Ji et al., 2012; Kim et al., 2011). In a recent study of self-reported ADHD in children, increasing PFHxS levels were associated with increasing prevalence of ADHD (adjusted odds ratio of 1.59) (Stein and Savitz, 2011).

- Reproduction A recent study evaluated the possible association between PFC exposure and biomarkers of male reproductive health in a larger cross country population including 588 men from Greenland $(\mathrm{n}=106)$, Poland $(\mathrm{n}=189)$ and Ukraine $(\mathrm{n}=203)$ (Toft et al., 2012). For PFHxS a 35\% (95\% CI: 1; 70\%) lower proportion of normal sperm were found at the highest tertile compared with the first, and a non-significant decrease in the proportion of normal sperm was also observed at the second tertile.

- Lipid metabolism and cholesterol In the cross-sectional study with NHANES data (Nelson et al., 2010) an inverse relationship between exposure to $\mathrm{PFHxS}$ and total cholesterol levels were observed. Another study from NHANES data (1999-2000 and 2003-2004) examined the relationship between PFCs and components of the metabolic syndrome in participants (Lin et al., 2009). They found no associations for PFHxS in adults.

- Developmental effects

A British study found that girls born to mothers with maternal serum concentrations of PFHxS in the upper tertile weighed less (-108 g; 95\% CI: -206 to -10 ) at birth compared with girls born to mothers with serum concentrations in the lower tertile (Maisonet et al., 2012). 
Two other smaller Canadian studies did not find any associations between maternal PFHxS levels and fetal weight and length of gestation (Hamm et al., 2010; Monroy et al., 2008).

A study examined the association between PFCs and ADHD among children in the US (Hoffman et al., 2010). Data from this NHANES study from 1999-2000 and 2003-2004 ( $\mathrm{n}=571$; age 12-17) showed significant increased odds of ADHD disease with higher serum PFHxS level (OR 1.06).

- Immune system

Two studies investigated the childhood infection (immunoglobulin E) and found no association to cord blood PFHxS in 2-year old Taiwanese boys ( $\mathrm{n}=$ (Wang et al., 2011) and newborn Japanese infants (Okada et al., 2012).

A recent prospective study of a birth cohort from the National Hospital in the Faroe Islands $(n=656)$ reported a small negative correlation with antibody response to tetanus and diphtheria booster immunizations at age 5 years with increasing serum PFHxS (Grandjean et al., 2012).

\subsubsection{PFHpS (C7), PFNS (C8) and PFDS (C10)}

No toxicology studies were found in animals or human.

\subsubsection{PFOS (C8)}

\section{Animal experimental studies}

PFOS is classified as Repr. 1B and Carc.2 (Harmonised classification Annex VI of Regulation (EC) No 1272/2008 (CLP Regulation)) (ECHA, 2008) and it was added to Annex B of the Stockholm Convention on Persistent Organic Pollutants in May 2009.

The toxicity of PFOS has been extensively studied, and numerous studies have been conducted including toxicological studies in multiple species. The results have been reviewed in an OECD report (2002) and Lau et al. (Lau et al., 2007; Lau et al., 2004). A summary of the important findings are given here.

Repeated-dose studies in rats and nonhuman primates have shown decreased body weight, hepatotoxicity, reduced cholesterol, and a steep dose-response curve for mortality. In a study performed by 3M, refereed in the OECD-report, male and female rats were exposed to PFOS in diet for 104 weeks $(0.5 \mathrm{ppm}-20 \mathrm{ppm})$. The study showed that PFOS induced a small increase in the incident of tumors in liver, and thyroid and 
mammary glands (OECD, 2006). The NOAEL for male and female was considered to be $0.5 \mathrm{ppm}$ and $2 \mathrm{ppm}$ in diet respectively, which corresponds to approximately $0.03 \mathrm{mg} / \mathrm{kg} /$ day and $0.15 \mathrm{mg} / \mathrm{kg} /$ day. PFOS has not been shown mutagenic in a variety of assays. Two-generation reproductive toxicity studies in rats showed neonatal mortality (Lau et al., 2004; Luebker et al., 2005a).

The maternal and developmental studies of PFOS in rats and mice showed both maternal and developmental toxicity. Pregnant SpragueDawley rats and CD-1 mice were given 1-20 mg/ kg/day from gestation day (GD) 2 to GD 20 and GD 1 to GD 17 respectively (Thibodeaux et al., 2003; Lau et al., 2003). The major findings on the mothers were a reduction in serum T4 and T3, without effects on TSH. The mice dam experienced a reduction in serum triglycerides and an elevation in liver weight at a dose of $1 \mathrm{mg} / \mathrm{kg} /$ day. $50 \%$ of the newborn rats and mice died within 24 hours when prenatally exposed to $3 \mathrm{mg} / \mathrm{kg} /$ day and $10 \mathrm{mg} / \mathrm{kg} /$ day respectively. Serum T4 levels were suppressed in the PFOS-treated rat pups, although T3 and TSH levels were not altered. Delays in growth and development were observed (Lau et al., 2003). Luebker et al. (2005b) showed that maternal exposure up to $1.6 \mathrm{mg} / \mathrm{kg} /$ day was a critical dose leading to approximately $50 \%$ mortality among prenatally exposed pups within 4 days after delivery.

A two-generation reproduction toxicity study in rats showed no effects on reproduction in F0 females or their fetuses (on mating, estrous cycling and fertility) (Luebker et al., 2005a). However, reproductive outcome, such as decreased length of gestation, number of implantation sites, and increased numbers of dams with stillborn pups or with all pups dying on LDs 1-4, was affected at $3.2 \mathrm{mg} /(\mathrm{kg}$ day) and neonatal toxicity, as demonstrated by reduced survival and body-weight gain, occurred at a maternal dose of $1.6 \mathrm{mg} / \mathrm{kg} /$ day (Luebker et al., 2005a).

In a series of studies pregnant mice have been exposed to PFOS in order to evaluate the behavioral effects on the offspring. The studies are summarized by Mariussen (2012). Neonatal exposure of PFOS at specific time points, at the period of high neuronal growth, was shown to induce behaviour effects in adult mice. The exposure appeared to involve an effect on the development of the cholinergic system.

In summary, in rodents PFOS is related to decreased body weight, liver toxicity, induction of PPAR-alpha, and has adverse reproductive effects at the fetal and at the neonatal level. PFOS increases developmental mortality and affectsa the thyroid system and may induce neurobehavioral effects, particularly in developmentally exposed animals. 


\section{Human studies (PFOS)}

Except for PFOS, there are very few data on the health effects of other PFSAs, probably due to lower concentrations in human blood. Since in most studies there was a high correlation between PFOS and PFOA, many of the health effects in the epidemiological studies that were observed and summarized under the PFOA section, is also mentioned below.

Data on liver function, serum cholesterol and thyroid hormone levels have been collected and associated with levels of PFOS in serum of occupationally exposed workers. However, we will not focus any further on this.

The epidemiological studies of general population are summarized in Table 14.

\section{- Cancer}

Grice et al. were unable to detect an association between occupational PFOS exposure and the occurrence of skin, breast, prostate, or intestinal cancer in workers at a PFC-producing company (Grice et al., 2007).

- Thyroid hormone

In Inuit adults $(\mathrm{n}=623)$, PFOS concentrations were negatively associated with TSH and total T3 and positively with free T4 concentrations (Dallaire et al., 2009). Maternal PFOS correlated negatively with fetal T3 (Kim et al., 2011). PFOS was shown to compete with $\mathrm{T} 4$ for binding sites on human transthyretin (Weiss et al., 2009), which may also lead to a reduction in total thyroid hormone concentrations in the blood. Also in children serum PFOS concentrations were positively correlated with total T4 concentration (Lopez-Espinosa et al., 2012). An earlier study of 15 mother-infant pairs in Japan reported no association between a median $2.5 \mathrm{ng} / \mathrm{mL}$ cord blood concentration of PFOS, approximately $13 \%$ of the current study median, and newborn $\mathrm{TSH}_{\text {or }} \mathrm{FT}_{4}$ levels (Inoue et al., 2004). A recent cross-sectional analysis of self-reported thyroid disease in the NHANES ( $\mathrm{n}=3,974$ adults) reported a significant association for PFOS (PFOS $\geq 36.8 \mathrm{ng} / \mathrm{mL}$ ) and current treated thyroid disease in men (Melzer et al., 2010).

In summary, although the data are controversial, reported studies suggest negative effects of PFOS on the thyroid system and on the risk of ADHD.

- Reproduction

A recent study evaluated the possible association between PFC exposure and biomarkers of male reproductive health in a larger cross country population including 588 men from Greenland 
( $\mathrm{n}=106)$, Poland $(\mathrm{n}=189)$ and Ukraine $(\mathrm{n}=203)$ (Toft et al., 2012).

Higher PFC blood levels were found in Greenlandic Inuit. Cross

country for the three populations and for the two European

populations alone an increase in sperm cell morphology defects at increasing PFOS exposure was found, but not analyzing the Inuit population from Greenland alone. Cross country the proportion of morphological normal cells was 35\% lower [95\% confidence interval (CI): 4-66\%) for the third tertile of PFOS exposure as compared with the first. Thus, the study suggests a concentration dependent effect and maybe diet/race dependent effect of PFOS on semen quality. The results of the present study are supported by the findings of effects of PFCs on sperm morphology reported in a previous smaller Danish study (Joensen et al., 2009).

- Lipid metabolism and cholesterol No explicit changes in liver enzymes, cholesterol, or lipoproteins in serum could be detected in the serum of workers with PFOS concentrations below $6 \mathrm{mg} / \mathrm{L}$ (Olsen et al., 1999). A study from NHANES data (1999-2000 and 2003-2004) examined the relationship between PFCs and components of the metabolic syndrome in participants (Lin et al., 2009). They found that in adults, increased serum PFOS concentrations were associated with increased blood insulin, insulin resistance status, beta-cell function, increased serum HDL cholesterol.

- Developmental effects Some studies have reported inverse associations between PFOS and birth weight. In a study from US $(n=293)$ cord blood PFOS levels was significantly associated with decreases in birth weight and size but not newborn length and gestational age (Apelberg et al., 2007). Data from a subgroup of the Norwegian Mother and Child Cohort study ( $\mathrm{n}=901$ women; 2003-2004) showed slightly lower birth weight among infants born to mothers with the highest plasma levels of PFOS (Whitworth et al., 2012a).

A British study found that girls born to mothers with maternal serum concentrations of PFOS in the upper tertile weighed less ( $-140 \mathrm{~g}$ (95\%CI: -238 to -42 )) at birth compared with girls born to mothers with serum concentration in the lower tertile (Maisonet et al., 2012). Also in a study from Japan ( $\mathrm{n}=428)$, maternal PFOS levels correlated negatively with birth weight $(-148.8 \mathrm{~g}(95 \% \mathrm{CI}$ : -297.0 to -0.5),particularly in female infants. (Washino et al, 2009). In contrast, in the Danish National Birth Cohort study (DNBC) ( $\mathrm{n}=$ 1,400 mother/child), maternal PFOS was not associated with birth 
weight and risk of premature birth (Fei et al., 2007), and suggested that the associations might be related to PFOA.

Two other smaller Canadian studies did also not find any association between maternal PFOS and fetal weight and length of gestation (Hamm et al., 2010; Monroy et al., 2008).

Developmental milestones of children were examined in the substudy of the Danish National Birth Cohort $(n=1,400)$. No convincing associations were found beside that children who were born to mothers with higher PFOS levels were slightly more likely to start sitting without support at a later age (Fei et al., 2008). Another study did examine the association between PFCs and ADHD among children (Hoffman et al., 2010). Data from this NHANES study from 19992000 and 2003-2004 ( $\mathrm{n}=571$; age 12-17) showed significant increase odds of ADHD disease with higher serum PFOS (OR 1.03).

- Immune system A Danish study examined the prenatal exposure to PFOA (and PFOS) and the association with the infectious diseases in children (Fei et al., $2010)$ in the Danish National Birth Cohort ( $\mathrm{n}=1,400)$.

Hospitalizations for infection of the children were not associated with prenatal exposure to PFOS.

Two studies investigated the childhood infection (immunoglobulin E): cord blood PFOS was positively associated with cord blood serum IgE in 2-year old Taiwanese boys ( $\mathrm{n}=$ (Wang et al., 2011), but not in newborn Japanese infants (Okada et al., 2012).

A recent prospective study of birth cohort from the National Hospital in the Faroe Islands $(n=656)$ reported a negative correlation with antibody response to tetanus and diphtheria booster immunizations at age 5 years with increasing serum PFOS (and to a lesser extend PFHxS) (Grandjean et al., 2012). The conclusion was that elevated exposures to PFAAs were associated with reduced immune response to vaccination.

\subsubsection{Summary for PFSAs}

\section{Summary for animal toxicity upon PFSAs exposure}

Most studies reported are on PFOS exposures, and very few studies on effects by short chain substances have been conducted.

In rodents PFOS (C8) is related to decreased body weight, liver toxicity and is a PPAR-alpha inducer. PFOS has adverse reproductive effects at the fetal and the neonatal level. PFOS increases developmental mortality and affects the thyroid system. 
Upon exposure to PFBS (C4) at relatively high doses, mild effects at the liver, kidney and blood parameters were observed in rat studies.

Few studies on PFHxS (C6) toxicity in experimental animals have been conducted and need further research. A single study found hepatoxicity in rats, whereas no reproductive effect on fertility and offspring outcome was observed at relatively high PFHxS exposure.

\section{Summary for human health effects of PFSA}

Except for PFOS, only some Nordic and in general few data on the health effects of other PFSAs are found.

Occupational studies found an association between PFOS exposure and liver function, serum cholesterol, thyroid hormone levels and skin, breast, prostate, or intestinal cancer in workers at a PFC-producing company.

General population studies: A cross country study on European and Inuit populations found an increase in sperm cell morphology defects at increasing PFOS and PFHxS exposure, but not for the Inuit population from Greenland alone. This observation is supported by a smaller Danish study.

Reproductive and developmental effects: An inverse association between maternal PFOS (and for some studies PFHxS) and birth weight were reported for studies in US, Norway and England, but not significantly for the Danish birth cohort and the smaller studies in Canada. Concerning developmental milestones weak association was found in the Danish National birth cohort. In the arctic Inuit adult PFOS exposure was positively related to changes in thyroid factors but not for PFHxS. However, PFHxS levels were positively related to ADHD prevalence in children. Thus, although controversial data, the reported studies suggest negative effects of PFOS on the thyroid system and PFHxS as a risk factor in development of ADHD. In support to the Inuit study a US study found a relationship between the serum PFOS and PFHxS levels at age 12-17 and the risk of ADHD. However, further studies are needed to elucidate the possible risks.

A single study reported a link between PFOS exposure and metabolic syndrome with effects such as increased blood insulin, insulin resistance status, beta-cell function, increased serum HDL cholesterol. In addition, a cross-sectional study in the US found an inverse relationship between exposure to PFHxS and total cholesterol levels.

A few studies indicated an effect of PFOS on the immune system: In the Faroe Islands, a negative effect on the vaccination response in children at 5-7 year; and a Japanese study found an association between childhood infection (immunoglobulin E) and cord blood PFOS levels in 2-year old boys. However, no significant association between prenatal exposure to PFOS and infectious diseases in children in the Danish National Birth Cohort was found. 


\subsection{FTOH (fluorotelomer alcohols)}

Limited information is currently available on the toxicological effects and the risks of FTOHs in experimental animals and humans. However, these compounds are metabolically converted to PFCAs including PFOA and therefore may be associated with the induction of hepatic peroxisome proliferation and acyl-CoA oxidase (ACOX) activity. There is a proposed classification under CLP regulations for 8:2 FTOH by Norway in 2012 as reproductive toxicant.

\subsubsection{Toxicity of FTOH in experimental animals}

Fluorotelomer alcohols have been shown to metabolize into PFCAs in rodents (Fasano et al., 2009; Kudo et al., 2005). Therefore, they may potentially have the same health effects as PFOA and some other PFCAs (PFNA and PFHpA).

Dietary administration of 8:2 FTOH to mice (7-28 days) resulted in liver enlargement in a dose-dependent manner. Peroxisomal acyl-CoA oxidase was induced by these treatments (Kudo et al., 2005). Five metabolites (PFOA, PFNA, 8-2 telomer acid and two unidentified) were present in the liver and serum of the treated mice. The concentration of PFOA positively correlated to the activity of peroxisomal acyl-CoA oxidase in the liver of mice, which suggest that rather PFOA than 8:2 FTOH itself produced the effects.

In a 90-day oral repeated dose toxicity study (Ladics et al., 2008) the liver and kidney were target organs. Doses of $25 \mathrm{mg} / \mathrm{kg}$ or greater increased relative liver weight, hepatic $\beta$-oxidation (females only at 25 $\mathrm{mg} / \mathrm{kg}$, both sexes at $125 \mathrm{mg} / \mathrm{kg}$ ), and induced focal hepatic necrosis. No evidence of a neurotoxic response was reported. The NOAEL in this study was $5 \mathrm{mg} / \mathrm{kg}$.

In a reproductive toxicity study with FTOH mixture $[\mathrm{F}(\mathrm{CF} 2) \mathrm{xC} 2 \mathrm{H} 4 \mathrm{OH}$, $\mathrm{x}=3-6$; (27\% 8:2 FTOH)] by oral gavage at $0,25,100$ or $250 \mathrm{mg} / \mathrm{kg} / \mathrm{day}$, pup weights were reduced at $\geq 100 \mathrm{mg} / \mathrm{kg} /$ day (Mylchreest et al., 2005a); in the developmental part of the study $(0,50,200$, or $500 \mathrm{mg} / \mathrm{kg} /$ day), maternal bodyweight was reduced and there was an increase in fetal skeletal alterations at $200 \mathrm{mg} / \mathrm{kg} /$ day. There were no effects on oestrous cycle parameters sperm morphology and motility or epididymal sperm counts in the P1 generation. NOAEL for the FTOH mixture (27\% 8:2 FTOH) reproductive toxicity was $25 \mathrm{mg} / \mathrm{kg} /$ day based on litter size reduction. In another study Mylchreest et al. (2005b) investigated the developmental effects of 8:2 FTOH at the same doses as above. Increases in skeletal vari- 
ations were reported from $200 \mathrm{mg} / \mathrm{kg} \mathrm{bw} /$ day as for the FTOH mixture. Severe maternal toxicity was reported at $500 \mathrm{mg} / \mathrm{kg}$ bw/day including mortality. The NOAEL for both maternal and developmental toxicity was sat to be $200 \mathrm{mg} / \mathrm{kg} /$ day.

Overall, the liver appears to be the most sensitive target organ for 8:2 FTOH toxicity based on the available studies. However, no carcinogenic studies were available and the reproductive and developmental studies are insufficient to draw any conclusions. Based on the toxicity effects of PFOA (major serum metabolite of FTOH), there is proposed a classification for 8:2 FTOH equal to PFOA (http://echa.europa.eu/).

In an in vitro study investigating oxidative damage that may contribute to testicular toxicity, primary rat testicular cells were exposed to $8: 2$ FTOH and 6:2 FTOH. The study showed no significant increase in oxidative DNA damage (Lindeman et al., 2012).

In summary, fluorotelomer alcohols and/or there metabolites (e.g.PFOA) can induce liver toxicity in rodent, and the potency of endocrine disruption are demonstrated in in vitro cell culture and in vivo fish studies.

Gap of knowledge: Fluorotelomer unsaturated aldehydes (FTUALs) and acids (FTUCAs) are intermediate metabolites that form from the degradation of FTOHs. Their potential for toxicity is not yet defined and may be more significant compared to PFCAs. These intermediates can form adducts with glutathione (GSH) (Rand and Mabury, 2012).

\subsubsection{Human health effects of FTOH}

In vitro studies using human cells have reported following endocrine effects for fluorotelomer alchohols: 8:2 FTOH and 6:2 FTOH had potential estrogenic effects by inducing the proliferation of MCF-7 breast cancer cells in E-screen assay; by up-regulating of the estrogen receptor (Maras $M$ et al., 2006); by interacting with the human estrogen receptor alpha and beta in vitro (Ishibashi $\mathrm{H}$ et al., 2007). Moreover, 8:2 FTOH showed the capacity to decrease testosterone levels in the human adrenal corticocarcinoma cell line (Liu et al., 2010b) but did not interfere with androgen receptor activation (Rosenmai et al., 2012). The authors assumed that the effect was due to FTOH and not the metabolite PFOA, as the hormone profile of these two compounds did not uniformly match. 


\subsubsection{Summary on FTOH}

\section{FTOH Toxicity in experimental animals}

Few data on FTOH health effects in animal and humans are found.

In rodents the liver appears to be the most sensitive target organ for 8:2 FTOH toxicity. Futher studies on carcinogenesis, repoductive and developmental effects are needed.

The potency of endocrine disruption is demonstrated in in vitro studies. In vitro, oxidative damage were found in rat testicular cells exposed to $8: 2 \mathrm{FTOH}$ and 6:2 FTOH.

There are gaps of data for the intermediate metabolites of FTOH (fluorotelomer unsaturated aldehydes (FTUALs) and acids (FTUCAs)) that form from the degradation of FTOHs. Their potential for toxicity is not yet defined and may be more significant compared to PFCAs.

\section{Human health effects of FTOH}

No available human data for FTOH are found but in vitro studies using human cells have reported endocrine effects for the fluorotelomer alchohols 8:2 FTOH and 6:2 FTOH.

\subsection{FTS (fluorotelomer sulfonates).}

Acute and repeated-dose mammalian and aquatic toxicity has been reportefor 6:2 FTS. 26

\subsection{PAP/di-PAP (polyfluoroalkyl phosphate esters)}

\subsubsection{Toxicity in experimental animals}

In rats biotransformation of monoPAP and diPAP congeners to FTOH and PFCAs have been observed (D'Eon J and Mabury, 2011). The animals were dosed with a mixture of $4: 2,6: 2,8: 2$, and 10:2 monoPAP or diPAP chain lengths. Concentrations of the PAPs and PFCAs, as well as metabolic intermediates and phase II metabolites, were monitored over time in blood, urine, and feces. The diPAPs were bioavailable, with bioavailability de-

26 UNEP/POPS/POPRC.8/INF/17 
creasing as the chain length increased from 4 to 10 perfluorinated carbons. The monoPAPs were not absorbed from the gut. However, the PAP dosing concentrations at $50 \mathrm{mg} / \mathrm{kg}$ used in the studies (D'Eon J and Mabury, 2011; D'Eon and Mabury, 2007), where not toxic for the animals. No other toxicity data were found for PAP/di-PAP.

\subsubsection{Human health effects}

No epidemiological data regarding health effects were found for $\mathrm{PAP} /$ di-PAP.

In vitro assays using human cell cultures (H295R human adrenal cortico-carcinoma cells) showed that 8:2 diPAPS and 8:2 monoPAPS gave rise to decreases in androgens (testosterone, dehydroepiandrosterone, and androstenedione) in the steroidogenic pathway, indicating an affect of steroidogenesis (Rosenmai et al., 2012). 8:2 triPAPS, 10:2 diPAPS showed a less marked effect on androgens in the steroidogenesis assay and did not disrupt the binding to androgen receptor.

\subsection{Perfluoropolyethers (PEFPs)}

The general toxicity profile of perfluoropolyethers is reported low. A study evaluating the safety of PEFPs, tested the representative Fomblin $\mathrm{HC}$ in experimental animals for acute, subacute toxicity, in vitro mutagenicity and irritancy or sensitization on human skin (Malinverno et al., 1996). Daily oral administration $(1,000 \mathrm{mg} / \mathrm{kg} /$ day $)$ to rats over 28 days produced no significant reaction.

No other toxicity data were found.

\subsubsection{Summary for PAP/di-PAP (polyfluoroalkyl phosphate esters) and perfluoropolyethers (PFPEs)}

\section{Toxicity in experimental animals}

MonoPAP and di-PAP congeners are bioavailable in rats and biotransformed to FTOH and PFCAs. PAP doses up to $50 \mathrm{mg} / \mathrm{kg}$ was not toxic in the rat study. No other toxicity data were found for PAP/di-PAP. 


\section{Human health effects}

No epidemiological data regarding health effects were found for $\mathrm{PAP} /$ di-PAP.

In vitro assays using human cell cultures showed some endocrine disrupting potential by affecting the steroidogenesis.

Few data on PFPEs are reported. The general toxicity profile of PFPEs is low and no significant reaction in experimental animals was found for acute, subacute toxicity, in vitro mutagenicity and irritancy or sensitization on human skin.

No other toxicity data were found.

\subsection{Summary}

\subsubsection{Animal toxicity}

In general PFCAs and PFSAs affect the development, reproduction and immune system negatively; they decrease body weight, induce hepatoxicity, affect the endocrine system negatively including the sex hormone and thyroid hormone system; induces peroxisome proliferation believed to be one of the mechanisms behind tumor initiation and affects the immune- and hormone systems.

Hepatotoxicity: Hepatocytic hypertrophy effect in laboratory animals were reported for PFOS, PFHxS, PFBS, PFDA, PFNA, PFOA, PFHpA, PFHxA, and PFBA and is most likely associated with induced peroxisome proliferation.

Developmental Toxicity: Early pregnancy loss was noted with PFOA or PFBA exposure but only at very high doses, and the etiology of this effect is not clear. No fetal toxicity was observed after gestational exposure to PFBA or PFDA. Compared to long-chain PFAAs $(\geq \mathrm{C} 8)$, the short-chain chemicals are much less toxic to the developing animal, in part due to their faster rate of clearance. Thus, even at very high doses of PFBA (350 mg/kg, intended to match the body burden of PFOA), neither neonatal survival nor postnatal growth was compromised, although maternal hepatomegaly was detected (indicating the effectiveness of the PFBA dose regimen) and neonatal liver weight was transiently elevated. A similar lack of reproductive and developmental toxicity has been reported for PFHxA, PFBS and PFHxS.

Immunotoxic: In general adverse immunological outcomes were reported from exposure to PFOS, PFHxS, PFOA and PFNA. 
Endocrine disruption: In general, alterations of thyroid hormones and sex steroid hormones have been shown after exposure to primarily PFOS and PFOA, although PFDA induced reductions of thyroid hormones have also been reported. PFOA has been shown to decrease serum and testicular testosterone and increase serum estradiol in male rats, presumably via induction of the hepatic aromatase. PFOS, PFOA, and telomer alcohols have been shown to exhibit estrogenic activity in vivo models and to inhibit testicular steroidogenic enzymes. In addition, PFDoA has recently been shown to decrease testosterone synthesis in male rats and to decrease serum estradiol and gene expression of estrogen receptors in the female rats, possibly through oxidative stress pathways.

\subsubsection{Human epidemiological studies}

An array of studies, mainly cross sectional design in US has been conducted with the main focus on PFOS and PFOA (Table 13). The overall observations were an association between PFCAs, PFSAs and effects on liver parameters such as lipid profile, the reproductive system (e.g. menopause), the thyroid hormone system, and an increased risk of ADHD (PFHxS). Serum PFAAs were associated with altered glucose homeostasis, indicators of metabolic syndrome, and elevated liver enzymes; a positive association between serum PFOS, PFOA and PFNA and cholesterol level; a significant association of PFOS and PFOA with thyroid related diseases.

Also an array of population studies on developmental effects have been conducted of which only 4 out of 12 are Nordic studies (Table 14). An inverse relationship between in utero exposure to PFOS and PFOA and birth weight was reported. However, these data call for further studies.

Follow-up evaluations of infants and children in the Danish National Birth Cohort indicated no associations between prenatal exposure to PFAAs and risk of infectious diseases, developmental milestones, and behavioral and motor coordination problems. Whereas a study on the Faroe Islands birth cohort study showed that PFC levels inversely correlated to the vaccination response at age 5 . These data call for further studies.

Some reports suggest a relationship between PFOS, PFOA and/or PFHxS exposure and the risk of ADHD, but again these data call for further research.

A single Danish study found that in utero exposure to PFOA was positively associated with BMI at age 20 .

A recent British cohort study did not find an association between maternal PFAA exposure and altered age at menarche of their offspring. 
Five Nordic (out of seven) studies on PFCA and PFSA effects on reproductive factors have been conducted. The overall observations were: Time-to-pregnancy in pregnant women in the Danish National Birth Cohort was suggested to be associated with PFOA and PFOS exposure and cause a reduction of fecundity; high PFAA levels and reduced normal sperm in Danish men; fewer normal sperm in a cross sectional study (Poland, Sweden, Ukraine, Greenland) upon high PFAA (PFOS); no effect on age of menarche in a single UK study.

Few data on the population studies calls for further research on reproductive factors as TTP, fecundity / fertility and the mechanisms behind.

Two Nordic studies have focused on the effect of PFCA and PFSA on the immune system and found controtradictory data: in Denmark no association to hospitalization and exposure was found for PFOS and PFOA, whereas in the Faroe Islands a negative effect on children vaccination response was reported. Two other studies (Taiwan, Japan) have suggested a correlation between PFOS and PFOA exposure and effect on the immune system such as changes in IgE levels in infants and cord blood, whereas no relationship between allergy in infant and maternal exposure was found.

In a Danish study including women and men no association was found on PFOA and PFOS exposure and the risk of prostate, bladder, or liver cancer. However, a recent study showed a significant association between serum PFOA (sum of PFCs) and risk of breast cancer among Inuit.

Very few studies (no Nordic) suggesting a relationship between PFCA and PFSA exposure and lipid profile changes and cardiovascular deceases calls for further research. 
Table13. Results of human studies from exposure to PFOA-contaminated water in Ohio/West Virginia communities (C8 Health Project)

\begin{tabular}{|c|c|c|c|c|c|c|c|c|c|}
\hline $\begin{array}{l}\text { 1st. Author, Year, } \\
\text { Ref. }\end{array}$ & Location & Population & Design & $\begin{array}{l}\text { Sampling } \\
\text { date }\end{array}$ & No. & PFCs measured & Outcome & $\begin{array}{l}\text { Median } \\
\text { PFOS/PFOA conc. } \\
\text { (ng/ml) }\end{array}$ & Results \\
\hline $\begin{array}{l}\text { Emmett, } 2006 \\
\text { (Emmett } \\
\text { et al., 2006) }\end{array}$ & USA/ Ohio & $\begin{array}{l}53 \% \text { female; } \\
\text { age } 2,5-89 \\
\text { (median 50) }\end{array}$ & $\begin{array}{l}\text { cross- } \\
\text { sectional }\end{array}$ & ? & 371 & PFOA & health parameters & $\begin{array}{l}\text { PFOA: } 354 \mathrm{ng} / \mathrm{ml} \\
\text { (median) }\end{array}$ & $\begin{array}{l}\text { No associations between PFOA and liver } \\
\text { function, cholesterol, TSH, red } \\
\text { cell/white cell or platelet counts. }\end{array}$ \\
\hline $\begin{array}{l}\text { Steenland, } 2009 \\
\text { (Steenland } \\
\text { et al., 2009) }\end{array}$ & $\begin{array}{l}\text { USA/West } \\
\text { Virginia + } \\
\text { Ohio (C8) }\end{array}$ & adults $(<18)$ & $\begin{array}{l}\text { cross- } \\
\text { sectional }\end{array}$ & $\begin{array}{l}2005- \\
2006\end{array}$ & 46,294 & PFOS, PFOA & serum lipids & $\begin{array}{l}\text { PFOS: } 20 \text {; PFOA: } \\
27 \mathrm{ng} / \mathrm{ml} \text { (median) }\end{array}$ & $\begin{array}{l}\text { Positive associations- No association to } \\
\mathrm{HDL}\end{array}$ \\
\hline $\begin{array}{l}\text { MacNiel, } 2009 \\
\text { (MacNeil et al., } \\
\text { 2009) }\end{array}$ & USA/Ohio & adults & $\begin{array}{l}\text { cross- } \\
\text { sectional }\end{array}$ & $\begin{array}{l}2005- \\
2006\end{array}$ & 54,468 & PFOA & diabetes II & PFOA: $28 \mathrm{ng} / \mathrm{ml}$ & $\begin{array}{l}\text { No association between PFOA and typell } \\
\text { diabetes }\end{array}$ \\
\hline $\begin{array}{l}\text { Frisbee, } 2010 \\
\text { (Frisbee et al., 2010) }\end{array}$ & $\begin{array}{l}\text { USA/Ohio } \\
\text { (C8) }\end{array}$ & $\begin{array}{l}\text { children and } \\
\text { adolescents } \\
(1-17.9)\end{array}$ & $\begin{array}{l}\text { cross- } \\
\text { sectional }\end{array}$ & $\begin{array}{l}2005- \\
2006\end{array}$ & 12,476 & PFOS, PFOA & $\begin{array}{l}\text { Serum lipids (total, } \\
\mathrm{HDL} \text {, and LDL } \\
\text { cholesterol, and } \\
\text { fasting triglycerides }\end{array}$ & $\begin{array}{l}\text { PFOS: 20.7; PFOA: } \\
32.6 \mathrm{ng} / \mathrm{ml} \text { (medi- } \\
\text { an) } 1-12 \text { years }\end{array}$ & $\begin{array}{l}\text { PFOA associated with increased total and } \\
\text { LDL cholesterol, and PFOS associated } \\
\text { with increased total, HDL, and LDL } \\
\text { cholesterol. }\end{array}$ \\
\hline $\begin{array}{l}\text { Steenland, } 2010 \\
\text { (Steenland et al., } \\
\text { 2010b) }\end{array}$ & $\begin{array}{l}\text { USA/ } \\
\text { West } \\
\text { Virginia + } \\
\text { Ohio (C8) }\end{array}$ & $\begin{array}{l}\text { adults } \geq 20 \\
\text { years }\end{array}$ & $\begin{array}{l}\text { cross- } \\
\text { sectional }\end{array}$ & $\begin{array}{l}2005- \\
2006\end{array}$ & 54,951 & PFOS, PFOA & uric acid & $\begin{array}{l}\text { PFOS: } 20.2 ; \text { PFOA: } \\
27.9 \mathrm{ng} / \mathrm{ml}\end{array}$ & positive association with hyperuricemia \\
\hline $\begin{array}{l}\text { Stein, } 2011 \text { (Stein } \\
\text { and Savitz, 2011) }\end{array}$ & $\begin{array}{l}\text { USA/Ohio } \\
\text { (C8) }\end{array}$ & $\begin{array}{l}\text { children (5- } \\
\text { 18) }\end{array}$ & $\begin{array}{l}\text { cross- } \\
\text { sectional }\end{array}$ & $\begin{array}{l}2005- \\
2006\end{array}$ & 10,546 & $\begin{array}{l}\text { PFOS, PFOA, } \\
\text { PFHxS, PFNA } \\
\text { (detectable) }\end{array}$ & ADHD and PFCs & $\begin{array}{l}\text { PFOS: } 20.2 \mathrm{ng} / \mathrm{ml} \text {; } \\
\text { PFOA } 28.2 \mathrm{ng} / \mathrm{mL} \\
\text { (median) }\end{array}$ & positive association for PFHXS \\
\hline
\end{tabular}




\begin{tabular}{|c|c|c|c|c|c|c|c|c|c|}
\hline $\begin{array}{l}\text { 1st. Author, Year, } \\
\text { Ref. }\end{array}$ & Location & Population & Design & $\begin{array}{l}\text { Sampling } \\
\text { date }\end{array}$ & No. & PFCs measured & Outcome & $\begin{array}{l}\text { Median } \\
\text { PFOS/PFOA conc. } \\
\text { (ng/ml) }\end{array}$ & Results \\
\hline $\begin{array}{l}\text { Knox, } 2011 \\
\text { (Knox et al., 2011a) }\end{array}$ & $\begin{array}{l}\text { USA/Ohio } \\
\text { (C8) }\end{array}$ & adults $(\mathrm{M}+\mathrm{F})$ & $\begin{array}{l}\text { cross- } \\
\text { sectional }\end{array}$ & $\begin{array}{l}2005- \\
2006\end{array}$ & 52,296 & PFOS, PFOA & Thyroid function & $\begin{array}{l}\text { Female: PFOS: } \\
\text { 17.3; PFOA: } 52.6 \\
\text { (mean)- Male: } \\
\text { PFOS: 24.8; PFOA: } \\
91 \text { (mean) }\end{array}$ & $\begin{array}{l}\text { PFOA and PFOS associated with eleva- } \\
\text { tions in serum thyroxine and reduction in } \\
\text { T3 uptake. }\end{array}$ \\
\hline $\begin{array}{l}\text { Knox, } 2011 \\
\text { (Knox et al., 2011b) }\end{array}$ & $\begin{array}{l}\text { USA/Ohio } \\
\text { (C8) }\end{array}$ & $\begin{array}{l}\text { women }(18- \\
65)\end{array}$ & $\begin{array}{l}\text { cross- } \\
\text { sectional }\end{array}$ & $\begin{array}{l}2005- \\
2006\end{array}$ & 25,957 & PFOS, PFOA & menopause & $\begin{array}{l}\text { PFOS: } 15-21.5 ; \\
\text { PFOA: } 17.6-32.5 \\
\text { ng/ml (median) }\end{array}$ & $\begin{array}{l}\text { perimenopausal women: experience } \\
\text { menopause if they have high serum } \\
\text { concentrations of PFOS and PFOA }\end{array}$ \\
\hline $\begin{array}{l}\text { Gallo, } 2012 \\
\text { (Gallo et al., 2012) }\end{array}$ & $\begin{array}{l}\text { USA/Ohio } \\
\text { (C8) }\end{array}$ & adults & $\begin{array}{l}\text { cross- } \\
\text { sectional }\end{array}$ & $\begin{array}{l}2005- \\
2006\end{array}$ & 47,092 & PFOS, PFOA & $\begin{array}{l}\text { Liver function } \\
\text { (enzymes) }\end{array}$ & $\begin{array}{l}\text { PFOS: 20.3; PFOA: } \\
28 \mathrm{ng} / \mathrm{ml} \text { (median) }\end{array}$ & $\begin{array}{l}\text { positive association between PFOA and } \\
\text { PFOS concentrations and serum ALT level }\end{array}$ \\
\hline $\begin{array}{l}\text { Lopez-Espinosa, } \\
2012 \\
\text { (Lopez-Espinosa } \\
\text { et al., 2012) }\end{array}$ & US/ Ohio & $\begin{array}{l}\text { children (1- } \\
\text { 17) }\end{array}$ & $\begin{array}{l}\text { cross- } \\
\text { sectional }\end{array}$ & $\begin{array}{l}2005- \\
2006\end{array}$ & 10.725 & $\begin{array}{l}\text { PFOA, PFOS, } \\
\text { and PFNA }\end{array}$ & $\mathrm{TSH}, \mathrm{TT} 4$ & $\begin{array}{l}\text { PFOS:20; } \\
\text { PFOA:29,3 ng/ml }\end{array}$ & $\begin{array}{l}\text { Associations of serum PFOS and PFNA } \\
\text { with thyroid hormone levels and of } \\
\text { serum PFOA and hypothyroidism. }\end{array}$ \\
\hline
\end{tabular}


Table14. Epidemiological studies on adverse human health effects related to PFCA and PFSA exposures (general population)

\begin{tabular}{|c|c|c|c|c|c|c|c|c|c|}
\hline Author, Year, Ref. & Location & Population & Design & $\begin{array}{l}\text { Sampling } \\
\text { date }\end{array}$ & No. & PFCs measured & Outcome & $\begin{array}{l}\text { Median } \\
\text { PFOS/PFOA conc. } \\
\text { (ng/ml) }\end{array}$ & Results \\
\hline \multicolumn{10}{|c|}{ Developmental outcome } \\
\hline $\begin{array}{l}\text { Fei, } 2007 \\
\text { (Fei et al., 2007) }\end{array}$ & Denmark & $\begin{array}{l}\text { Pregnant/ } \\
\text { infant } \\
\text { (DNBC) }\end{array}$ & $\begin{array}{l}\text { prospective } \\
\text { follow-up }\end{array}$ & $\begin{array}{l}1996- \\
2002\end{array}$ & 1,400 & $\begin{array}{l}\text { PFOS, PFOA } \\
\text { (measured) }\end{array}$ & Fetal growth & $\begin{array}{l}\text { PFOS: } 35.3 \text { PFOA: } \\
5.6 \text { (mean) }\end{array}$ & PFOA inversely associated with BW \\
\hline $\begin{array}{l}\text { Fei, } 2008 \\
\text { (Fei et al., 2008) }\end{array}$ & $\begin{array}{l}\text { Denmark } \\
\text { (DNBC) }\end{array}$ & $\begin{array}{l}\text { Mother/ } \\
\text { child }\end{array}$ & prospective & $\begin{array}{l}1996- \\
2002\end{array}$ & 1,400 & PFOS, PFOA & $\begin{array}{l}\text { Developmental } \\
\text { milestones }\end{array}$ & $\begin{array}{l}\text { PFOS: } 34.4 \text { PFOA: } \\
5.4\end{array}$ & $\begin{array}{l}\text { Higher PFOS in mothers associated to } \\
\text { childs later start at sitting }\end{array}$ \\
\hline $\begin{array}{l}\text { Fei and Olsen, } 2011 \\
\text { (Fei and Olsen, } \\
\text { 2011) }\end{array}$ & $\begin{array}{l}\text { Denmark } \\
\text { (DNBC) }\end{array}$ & $\begin{array}{l}\text { Mother/ } \\
\text { child }\end{array}$ & prospective & $\begin{array}{l}1996- \\
2002\end{array}$ & 1,400 & PFOS, PFOA & ADHD & $\begin{array}{l}\text { PFOS: } 34.4 \text { PFOA: } \\
5.4\end{array}$ & $\begin{array}{l}\text { No association between higher SDQ } \\
\text { scores and maternal levels of PFOS or } \\
\text { PFOA; no association with motor coordi- } \\
\text { nation disorders. }\end{array}$ \\
\hline $\begin{array}{l}\text { Whitworth, } 2012 \\
\text { (Whitworth } \\
\text { et al., 2012a) }\end{array}$ & Norway & $\begin{array}{l}\text { pregnant } \\
\text { women } \\
\text { (MoBa) }\end{array}$ & & $\begin{array}{l}2003- \\
2004\end{array}$ & 910 & PFOA, PFOS & BW & $\begin{array}{l}\text { PFOS: } 19.3 \\
\text { PFOA: } 3.7 \\
\text { (median) }\end{array}$ & $\begin{array}{l}\text { Lower adjusted BW among infants born to } \\
\text { women with the highest plasma levels of } \\
\text { PFOA and PFOS }\end{array}$ \\
\hline $\begin{array}{l}\text { Inoue, } 2004 \\
\text { (Inoue et al., 2004) }\end{array}$ & $\begin{array}{l}\text { Japan/ } \\
\text { Hokkaido }\end{array}$ & $\begin{array}{l}\text { Pregnant } \\
\text { (17-37 age) }\end{array}$ & $\begin{array}{l}\text { cross- } \\
\text { sectional }\end{array}$ & 2003 & 15 & $\begin{array}{l}\text { PFOS, PFOA, } \\
\text { PFOSA }\end{array}$ & $\begin{array}{l}\text { BW/thyroid } \\
\text { function (T4, TSH) }\end{array}$ & $\begin{array}{l}\text { PFOS: } 8.1 \\
\text { PFOA: } 0.7\end{array}$ & No association \\
\hline $\begin{array}{l}\text { Apelberg, } 2007 \\
\text { (Apelberg } \\
\text { et al., 2007) }\end{array}$ & $\begin{array}{l}\text { US/ } \\
\text { Maryland }\end{array}$ & $\begin{array}{l}\text { pregnant } \\
\text { /infants }\end{array}$ & $\begin{array}{l}\text { cross- } \\
\text { sectional }\end{array}$ & $\begin{array}{l}2004- \\
2005\end{array}$ & 293 & PFOA, PFOS & $\begin{array}{l}\text { Fetal growth (BW } \\
\text { and size) }\end{array}$ & $\begin{array}{l}\text { PFOS: } 5 \\
\text { PFOA: } 1.6\end{array}$ & $\begin{array}{l}\text { Neg. association with BW, ponderal index, } \\
\text { head circumference }\end{array}$ \\
\hline $\begin{array}{l}\text { Monroy, } 2008 \\
\text { (Monroy } \\
\text { et al., 2008) }\end{array}$ & Canada & Pregnant & $\begin{array}{l}\text { cross- } \\
\text { sectional }\end{array}$ & $\begin{array}{l}2004- \\
2005\end{array}$ & 101 & $\begin{array}{l}\text { PFOA, PFOS, } \\
\text { PFDeA, PFHxS, } \\
\text { PFHpA, PFNA, }\end{array}$ & BW & $\begin{array}{l}\text { PFOS: } 16.6 \text { PFOA: } \\
2.13\end{array}$ & No association with BW \\
\hline
\end{tabular}




\begin{tabular}{|c|c|c|c|c|c|c|c|c|c|}
\hline Author, Year, Ref. & Location & Population & Design & $\begin{array}{l}\text { Sampling } \\
\text { date }\end{array}$ & No. & PFCs measured & Outcome & $\begin{array}{l}\text { Median } \\
\text { PFOS/PFOA conc. } \\
\text { (ng/ml) }\end{array}$ & Results \\
\hline $\begin{array}{l}\text { Hamm, 2010 } \\
\text { (Hamm et al., 2010) }\end{array}$ & Canada & Pregnant & $\begin{array}{l}\text { cross- } \\
\text { sectional }\end{array}$ & $\begin{array}{l}2005- \\
2006\end{array}$ & 252 & $\begin{array}{l}\text { PFOA, PFOS, } \\
\text { PFH } \times \text { S }\end{array}$ & fetal growt & $\begin{array}{l}\text { PFOS: } 35 \\
\text { PFOA: } 1.5\end{array}$ & $\begin{array}{l}\text { No correlations with BW, GSA, and other } \\
\text { birth parameters }\end{array}$ \\
\hline $\begin{array}{l}\text { Washino, } 2009 \\
\text { (Washino } \\
\text { et al., 2009) }\end{array}$ & Japan & Pregnant & prospective & $\begin{array}{l}2002- \\
2005\end{array}$ & 428 & PFOA, PFOS & $\mathrm{BW}$ and size & $\begin{array}{l}\text { PFOS: } 5.2 \\
\text { PFOA: } 1.3\end{array}$ & $\begin{array}{l}\text { PFOS negatively correlated with BW } \\
\text { (girls); no association with PFOA }\end{array}$ \\
\hline $\begin{array}{l}\text { Hoffman, } 2010 \\
\text { (Hoffman } \\
\text { et al., 2010) }\end{array}$ & $\begin{array}{l}\text { US } \\
\text { (NHANES) }\end{array}$ & $\begin{array}{l}\text { children (12- } \\
\text { 15) }\end{array}$ & $\begin{array}{l}\text { cross- } \\
\text { sectional }\end{array}$ & $\begin{array}{l}\text { 1999- } \\
2000 ; \\
2003- \\
2004\end{array}$ & 571 & $\begin{array}{l}\text { PFOA, PFNA, } \\
\text { PFOS, PFHXS }\end{array}$ & ADHD & $\begin{array}{l}\text { PFOS: } 22.6 \\
\text { PFOA: } 4.4\end{array}$ & $\begin{array}{l}\text { Positive relationship between parent- } \\
\text { reported ADHD and serum PFOS, PFOA, } \\
\text { and PFHXS }\end{array}$ \\
\hline $\begin{array}{l}\text { Gump, } 2011 \\
\text { (Gump et al., 2011) }\end{array}$ & USA & Child & $\begin{array}{l}\text { cross } \\
\text { sectional }\end{array}$ & ? & 83 & $\begin{array}{l}11 \text { PFCS: PFOS, } \\
\text { PFH xS, PFBS, } \\
\text { PFDS, } \\
\text { PFOSA,PFHpA, } \\
\text { PFOA, PFNA, } \\
\text { PFDA, PFUnDA, } \\
\text { PFDoDA }\end{array}$ & $\begin{array}{l}\text { impaired response } \\
\text { inhibition }\end{array}$ & $\begin{array}{l}\text { PFOS: } 8.79 \\
\text { PFOA: } 3.28\end{array}$ & $\begin{array}{l}\text { Higher levels of blood PFOS, PFNA, PFDA, } \\
\text { PFHXS, and PFOSA associated with shorter } \\
\text { IRTs (children's impulsivity) }\end{array}$ \\
\hline $\begin{array}{l}\text { Chen, } 2012 \\
\text { (Chen et al., 2012) }\end{array}$ & Taiwan & $\begin{array}{l}\text { mother- } \\
\text { infant (TBPS) }\end{array}$ & & $\begin{array}{l}2004- \\
2005\end{array}$ & 429 & $\begin{array}{l}\text { PFOA, PFOS, } \\
\text { PFNA, and } \\
\text { PFUA }\end{array}$ & $\begin{array}{l}\text { GSA, BW, head } \\
\text { circumference }\end{array}$ & $\begin{array}{l}\text { PFOS: } 5.94 \\
\text { PFOA: } 1.84\end{array}$ & $\begin{array}{l}\text { PFOS inversely associated with GSA, birth } \\
\text { weight, and head circumference }\end{array}$ \\
\hline $\begin{array}{l}\text { Maisonet, } 2012 \\
\text { (Maisonet } \\
\text { et al., 2012) }\end{array}$ & UK & $\begin{array}{l}\text { singelton } \\
\text { girls (ALSPA } \\
\text { cohort) }\end{array}$ & & $\begin{array}{l}1991- \\
1992\end{array}$ & 447 & $\begin{array}{l}\text { PFOS, PFOA, } \\
\text { PFHXS }\end{array}$ & $\begin{array}{l}\text { BW, } \\
\text { weight for age. }\end{array}$ & $\begin{array}{l}\text { PFOS: } 19.6 \\
\text { PFOA: } 3.7\end{array}$ & $\begin{array}{l}\text { PFOS negatively associated with girls } \\
\text { weight at birth; No associations with } \\
\text { PFOA and PFHXS }\end{array}$ \\
\hline
\end{tabular}




\begin{tabular}{|c|c|c|c|c|c|c|c|c|c|}
\hline Author, Year, Ref. & Location & Population & Design & $\begin{array}{l}\text { Sampling } \\
\text { date }\end{array}$ & No. & PFCs measured & Outcome & $\begin{array}{l}\text { Median } \\
\text { PFOS/PFOA conc. } \\
\text { (ng/ml) }\end{array}$ & Results \\
\hline \multicolumn{10}{|l|}{ Thyroid function } \\
\hline $\begin{array}{l}\text { Dallaire, } 2009 \\
\text { (Dallaire } \\
\text { et al., 2009) }\end{array}$ & Nunavik & Inuit adults & $\begin{array}{l}\text { cross- } \\
\text { sectional }\end{array}$ & 2004 & 623 & PFOS & Thyroid function & PFOS: 18.28 (GM) & $\begin{array}{l}\text { PFOS concentrations were negatively } \\
\text { associated with TSH, tT3 and TBG and } \\
\text { positively with } \mathrm{FT} 4 \text { concentrations }\end{array}$ \\
\hline $\begin{array}{l}\text { Bloom, } 2010 \\
\text { (Bloom et al., 2010) }\end{array}$ & USA/NY & $\begin{array}{l}\text { sportfish } \\
\text { anglers }\end{array}$ & $\begin{array}{l}\text { prospec- } \\
\text { tive }\end{array}$ & $\begin{array}{l}1995- \\
1997\end{array}$ & 31 & $\begin{array}{l}\text { PFDA, PFNA, } \\
\text { PFHAA, PFHxS, } \\
\text { PFOA, PFOS, } \\
\text { PFOSA, PFUnDA }\end{array}$ & T4. TSH & $\begin{array}{l}\text { PFOS: } 19.6 \text { PFOA: } \\
1.3\end{array}$ & $\begin{array}{l}\text { No associations between PFCs exposures } \\
\text { and thyroid function }\end{array}$ \\
\hline $\begin{array}{l}\text { Melzer, } 2010 \\
\text { (Melzer et al., 2010) }\end{array}$ & USA & $\begin{array}{l}\text { men and } \\
\text { women }\end{array}$ & & $\begin{array}{l}1999- \\
2006\end{array}$ & $\begin{array}{l}3966 \\
(52 \% \\
\text { women) }\end{array}$ & PFOA, PFOS & Thyroid diseases & $\begin{array}{l}\text { PFOS: } 19.14 \text { PFOA: } \\
\text { 3.77 (Female) } \\
\text { PFOS: } 25.08 \text { PFOA: } \\
4.91 \text { (men) (GM) }\end{array}$ & $\begin{array}{l}\text { Higher concentrations of serum PFOA and } \\
\text { PFOS are associated with current thyroid } \\
\text { disease }\end{array}$ \\
\hline $\begin{array}{l}\text { Kim, } 2011 \\
\text { (Kim et al., 2011) }\end{array}$ & $\begin{array}{l}\text { Korea/ } \\
\text { soul }\end{array}$ & $\begin{array}{l}\text { Mother/ } \\
\text { child }\end{array}$ & $\begin{array}{l}\text { cross } \\
\text { sectional }\end{array}$ & $\begin{array}{l}2008- \\
2009\end{array}$ & 44 & $\begin{array}{l}13 \text { PFCs: PFHxS, } \\
\text { PFHpS, PFOS, } \\
\text { PFDS, PFOA, } \\
\text { PFNA, PFDA, } \\
\text { PFUnDA, } \\
\text { PFDoDA, } \\
\text { PFTrDA PFTe- } \\
\text { DA, MePFOSAA, } \\
\text { EtPFOSAA. }\end{array}$ & $\begin{array}{l}\text { Thyroid hormone; } \\
\text { Bith weight }\end{array}$ & $\begin{array}{l}\text { PFOS: } 2.72 \\
\text { PFOA: } 1.46\end{array}$ & $\begin{array}{l}\text { Fetal PFOS, PFOA, PFTrDA and maternal } \\
\text { PFTrDA were correlated with fetal total T4 } \\
\text { concentrations (before adjustment). After } \\
\text { adjustment: negative correlations be- } \\
\text { tween maternal PFOS and fetal T3, and } \\
\text { maternal PFTrDA and fetal T4 and T3. }\end{array}$ \\
\hline
\end{tabular}




\begin{tabular}{|c|c|c|c|c|c|c|c|c|c|}
\hline Author, Year, Ref. & Location & Population & Design & $\begin{array}{l}\text { Sampling } \\
\text { date }\end{array}$ & No. & PFCs measured & Outcome & $\begin{array}{l}\text { Median } \\
\text { PFOS/PFOA conc. } \\
\text { (ng/ml) }\end{array}$ & Results \\
\hline $\begin{array}{l}\mathrm{Ji}, 2012 \\
\text { (Ji et al., 2012) }\end{array}$ & $\begin{array}{l}\text { Korea/ } \\
\text { Siheung }\end{array}$ & $>12$ years & $\begin{array}{l}\text { cross- } \\
\text { sectional }\end{array}$ & 2008 & $\begin{array}{l}633 \\
(59 \% \\
\text { female) }\end{array}$ & $\begin{array}{l}13 \text { PFCs: PFHxS, } \\
\text { PFHpS, PFOS, } \\
\text { PFDS, PFOA, } \\
\text { PFNA, PFDA, } \\
\text { PFUnDA, } \\
\text { PFDoDA, } \\
\text { PFTrDA, } \\
\text { PFTeDA, } \\
\text { MePFOSAA, } \\
\text { EtPFOSAA. }\end{array}$ & $\begin{array}{l}\text { Thyroid function } \\
\text { (T4. TSH) }\end{array}$ & $\begin{array}{l}\text { PFOS: } 7.96 \\
\text { PFOA: } 2.74\end{array}$ & $\begin{array}{l}\text { PFTrDA negatively correlated with } \Pi 4 \\
\text { and positively with TSH }\end{array}$ \\
\hline \multicolumn{10}{|l|}{ Reproduction } \\
\hline $\begin{array}{l}\text { Joensen, } 2009 \\
\text { (Joensen } \\
\text { et al., 2009) }\end{array}$ & Denmark & men (19) & $\begin{array}{l}\text { cross } \\
\text { sectional }\end{array}$ & 2003 & 105 & $\begin{array}{l}10 \text { PFCs (C6 to } \\
\text { C13); PFHXS. } \\
\text { PFHpA. PFOA. } \\
\text { PFOS.PFOSA. } \\
\text { PFNA. PFDA. } \\
\text { PFUnA. } \\
\text { PFDoA. PFTrA }\end{array}$ & $\begin{array}{l}\text { Reproductive } \\
\text { hormones and } \\
\text { semen }\end{array}$ & $\begin{array}{l}\text { PFOS: } 24.5 \\
\text { PFOA: } 4.9\end{array}$ & $\begin{array}{l}\text { High PFAA levels were associated with } \\
\text { fewer normal sperm. }\end{array}$ \\
\hline $\begin{array}{l}\text { Fei, } 2009 \\
\text { (Fei et al., 2009) }\end{array}$ & Denmark & $\begin{array}{l}\text { pregnant } \\
\text { women }\end{array}$ & $\begin{array}{l}\text { prospec- } \\
\text { tive }\end{array}$ & $\begin{array}{l}1996- \\
2002\end{array}$ & 1,240 & PFOA. PFOS & TTP; Fecundity & $\begin{array}{l}\text { PFOS: } 33.7 \text { PFOA: } \\
5.3\end{array}$ & $\begin{array}{l}\text { Pos association to TTP and reduced } \\
\text { fecundity }\end{array}$ \\
\hline $\begin{array}{l}\text { Vestergaard, } 2012 \\
\text { (Vestergaard } \\
\text { et al., 2012) }\end{array}$ & Denmark & $\begin{array}{l}\text { Women }(18- \\
35)\end{array}$ & $\begin{array}{l}\text { prospec- } \\
\text { tive study }\end{array}$ & $\begin{array}{l}1992- \\
1995\end{array}$ & 222 & $\begin{array}{l}8 \text { PFCs: PFOS. } \\
\text { PFOA.PFHxS. } \\
\text { PFNA. PFDA. } \\
\text { MeFOSAA.EtF } \\
\text { OSAA and } \\
\text { FOSA }\end{array}$ & Fecundity & $\begin{array}{l}\text { PFOS: } 36 \\
\text { PFOA: } 5.6\end{array}$ & $\begin{array}{l}\text { No clear association between PFCs } \\
\text { and TTP }\end{array}$ \\
\hline
\end{tabular}




\begin{tabular}{|c|c|c|c|c|c|c|c|c|c|}
\hline Author, Year, Ref. & Location & Population & Design & $\begin{array}{l}\text { Sampling } \\
\text { date }\end{array}$ & No. & PFCs measured & Outcome & $\begin{array}{l}\text { Median } \\
\text { PFOS/PFOA conc. } \\
\text { (ng/ml) }\end{array}$ & Results \\
\hline $\begin{array}{l}\text { Whitworth, } 2012 \\
\text { (Whitworth et al., } \\
\text { 2012b) }\end{array}$ & Norway & $\begin{array}{l}\text { pregnant } \\
\text { women } \\
\text { (MoBa) }\end{array}$ & $\begin{array}{l}\text { case- } \\
\text { control }\end{array}$ & $\begin{array}{l}2003- \\
2004\end{array}$ & $\begin{array}{l}910(416 \\
\text { cases })\end{array}$ & PFOA. PFOS & TTP & $\begin{array}{l}\text { PFOS: } 13 \text { PFOA: } \\
2.2\end{array}$ & $\begin{array}{l}\text { PFOA and PFOS exposure at plasma levels } \\
\text { seen in the general population may } \\
\text { reduce fecundity; }\end{array}$ \\
\hline $\begin{array}{l}\text { Toft, 2012 } \\
\text { (Toft et al., 2012) }\end{array}$ & $\begin{array}{l}\text { GRL/PL/ukra } \\
\text { ine }\end{array}$ & Men & $\begin{array}{l}\text { cross } \\
\text { sectional }\end{array}$ & $\begin{array}{l}2002- \\
2004\end{array}$ & 196 & $\begin{array}{l}\text { PFHXS. PFOS. } \\
\text { PFOA. PFNA. } \\
\text { PFDA. PFUnDA } \\
\text { and PFDoDA }\end{array}$ & Semen quality & $\begin{array}{l}\text { PFOS: } 44.7 \\
\text { PFOA: } 4.5 \text { (GRL) } \\
\text { PFOS: } 18.5 \text { PFOA: } \\
\text { 4.8 (PL) PFOS:7.6 } \\
\text { PFOA: } 1.3 \\
\text { (Ukraine) }\end{array}$ & $\begin{array}{l}\text { Negative associations between PFOS } \\
\text { exposure and sperm morphology }\end{array}$ \\
\hline $\begin{array}{l}\text { Christensen, } 2011 \\
\text { (Christensen } \\
\text { et al., 2011) }\end{array}$ & UK & $\begin{array}{l}\text { ALSPA } \\
\text { cohort }\end{array}$ & $\begin{array}{l}\text { nested } \\
\text { case } \\
\text { control }\end{array}$ & $\begin{array}{l}1991- \\
1992\end{array}$ & $\begin{array}{l}218 \\
\text { cases; } \\
230 \\
\text { controls }\end{array}$ & $\begin{array}{l}8 \text { PFCS: incl. } \\
\text { PFOS. PFOA }\end{array}$ & Age of menarche & $\begin{array}{l}\text { PFOS: } 19.8 \text { PFOA: } \\
3.7\end{array}$ & No associations with age at menarche \\
\hline $\begin{array}{l}\text { Raymer, } 2011 \\
\text { (Raymer } \\
\text { et al., 2012) }\end{array}$ & US & Men & $\begin{array}{l}\text { cross- } \\
\text { sectional }\end{array}$ & $\begin{array}{l}2002- \\
2005\end{array}$ & 256 & PFOA. PFOS & $\begin{array}{l}\text { Semen quali- } \\
\text { ty/reproductive } \\
\text { hormones }\end{array}$ & $\begin{array}{l}\text { PFOS: } 32.3 \\
\text { PFOA: } 5.2\end{array}$ & $\begin{array}{l}\text { No association with sperm parameters. } \\
\text { pos correlation with LH }\end{array}$ \\
\hline \multicolumn{10}{|l|}{ Immune system } \\
\hline $\begin{array}{l}\text { Fei, } 2010 \\
\text { (Fei et al., 2010) }\end{array}$ & $\begin{array}{l}\text { Denmark } \\
\text { (DNBC) }\end{array}$ & $\begin{array}{l}\text { Mother/ } \\
\text { child }\end{array}$ & $\begin{array}{l}\text { prospec- } \\
\text { tive }\end{array}$ & $\begin{array}{l}1996- \\
2002\end{array}$ & 1,400 & PFOS. PFOA & $\begin{array}{l}\text { Hospitaliza- } \\
\text { tion/infection }\end{array}$ & $\begin{array}{l}\text { PFOS: } 34.4 \text { PFOA: } \\
5.4\end{array}$ & $\begin{array}{l}\text { No association between hospitalizaion } \\
\text { and PFCs }\end{array}$ \\
\hline $\begin{array}{l}\text { Grandjean, } 2012 \\
\text { (Grandjean } \\
\text { et al., 2012) }\end{array}$ & $\begin{array}{l}\text { Faroe } \\
\text { Iceland }\end{array}$ & $\begin{array}{l}\text { Mother/ } \\
\text { child }\end{array}$ & $\begin{array}{l}\text { prospec- } \\
\text { tive }\end{array}$ & $\begin{array}{l}1997- \\
2000\end{array}$ & 587 & $\begin{array}{l}\text { PFOS. PFOA. } \\
\text { PFHXS. PFNA. } \\
\text { PFDA }\end{array}$ & $\begin{array}{l}\text { Antibody conc. } \\
\text { after vaccination }\end{array}$ & $\begin{array}{l}\text { PFOS: } 27.3 \text { PFOA: } \\
3.20 \\
\text { (GM) }\end{array}$ & $\begin{array}{l}\text { PFOS and PFOA associated with lower } \\
\text { antibody responses to childhood im- } \\
\text { munizations at age } 5 \text {. PFDA positively } \\
\text { associated with the antibody concentra- } \\
\text { tion in blood }\end{array}$ \\
\hline
\end{tabular}




\begin{tabular}{|c|c|c|c|c|c|c|c|c|c|}
\hline Author, Year, Ref. & Location & Population & Design & $\begin{array}{l}\text { Sampling } \\
\text { date }\end{array}$ & No. & PFCs measured & Outcome & $\begin{array}{l}\text { Median } \\
\text { PFOS/PFOA conc. } \\
\text { (ng/ml) }\end{array}$ & Results \\
\hline $\begin{array}{l}\text { Wang, } 2011 \\
\text { (Wang et al., 2011) }\end{array}$ & Taiwan & children & $\begin{array}{l}\text { birth } \\
\text { cohort } \\
\text { study }\end{array}$ & 2004 & 244 & $\begin{array}{l}12 \text { PFCS: } \\
\text { detected PFOA. } \\
\text { PFOS. PFNA. } \\
\text { and PFHxS }\end{array}$ & Pediatric atopi & $\begin{array}{l}\text { PFOA: } 1.71 \\
\text { PFOS: } 5.50\end{array}$ & $\begin{array}{l}\text { PFOA and PFOS levels positively correlat- } \\
\text { ed with cord blood lgE levels }\end{array}$ \\
\hline $\begin{array}{l}\text { Okada, } 2012 \\
\text { (Okada et al., 2012) }\end{array}$ & $\begin{array}{l}\text { Japan / } \\
\text { Sapporo }\end{array}$ & $\begin{array}{l}\text { Pregnant } \\
\text { women/ } \\
\text { infant }\end{array}$ & $\begin{array}{l}\text { Cross- } \\
\text { sectional }\end{array}$ & $\begin{array}{l}2002- \\
2005\end{array}$ & 343 & PFOS, PFOA & $\begin{array}{l}\text { Allergies and } \\
\text { infectious diseases }\end{array}$ & $\begin{array}{l}\text { PFOS: } 5.2 \\
\text { PFOA: } 1.3\end{array}$ & $\begin{array}{l}\text { Among female infants, cord blood IgE } \\
\text { levels decreased significantly with high } \\
\text { maternal PFOA serum levels. No rela- } \\
\text { tionship was found between maternal } \\
\text { PFOS and PFOA serum levels and infant } \\
\text { allergies and infectious diseases at } 18 \\
\text { months of age. }\end{array}$ \\
\hline \multicolumn{10}{|l|}{ Cancer } \\
\hline $\begin{array}{l}\text { Eriksen, } 2009 \\
\text { (Eriksen et al., } \\
\text { 2009) }\end{array}$ & Denmark & $\begin{array}{l}\text { men and } \\
\text { women }\end{array}$ & $\begin{array}{l}\text { prospecti- } \\
\text { ve }\end{array}$ & $\begin{array}{l}1993- \\
1997\end{array}$ & $\begin{array}{l}1,240 \\
(90 \% \\
\text { women) }\end{array}$ & PFOA. PFOS & Cancer & $\begin{array}{l}\text { PFOS: } 35 \text { PFOA: } \\
6.8\end{array}$ & $\begin{array}{l}\text { No association to bladder, pancreatic or } \\
\text { liver cancer. Small increase in risk for } \\
\text { prostate cancer }\end{array}$ \\
\hline $\begin{array}{l}\text { Bonefeld-Jørgense, } \\
2011 \text { (Bonefeld- } \\
\text { Jorgensen et al., } \\
\text { 2011) }\end{array}$ & Greenland & Inuit women & $\begin{array}{l}\text { case- } \\
\text { control }\end{array}$ & $\begin{array}{l}2000- \\
2003\end{array}$ & $\begin{array}{l}31 \text { cases } \\
115 \\
\text { controls }\end{array}$ & $\begin{array}{l}\text { PFOS. PFOA. } \\
\text { PFHxS. PFOSA. } \\
\text { PFHpA. PFNA. } \\
\text { PFDA. PFUnA. } \\
\text { PFDoA. PFTrDA }\end{array}$ & Breast Cancer & $\begin{array}{l}\text { PFOS: } 45.6 \\
\text { PFOA: } 2.5\end{array}$ & $\begin{array}{l}\text { Higher PFC levels in breast cancer cases; } \\
\text { Pos odds ratio }\end{array}$ \\
\hline \multicolumn{10}{|c|}{ Lipid metabolisme /biochemicalparameters } \\
\hline $\begin{array}{l}\text { Halldorsson TI, } \\
2012 \text { (Halldorsson } \\
\text { et al., 2012) }\end{array}$ & Denmark & $\begin{array}{l}\text { Mother/ } \\
\text { child } \\
\text { (Aarhus BC) }\end{array}$ & $\begin{array}{l}\text { prospec- } \\
\text { tive cohort }\end{array}$ & $\begin{array}{l}1988- \\
1989\end{array}$ & 665 & 19 PFCS & BMI at 20 years & $\begin{array}{l}\text { PFOS: 21.5; PFOA: } \\
3.7\end{array}$ & $\begin{array}{l}\text { in utero exposure to PFOA positively } \\
\text { associated with anthropometry at } 20 \\
\text { years in female but not male offspring }\end{array}$ \\
\hline
\end{tabular}




\begin{tabular}{|c|c|c|c|c|c|c|c|c|c|}
\hline Author, Year, Ref. & Location & Population & Design & $\begin{array}{l}\text { Sampling } \\
\text { date }\end{array}$ & No. & PFCs measured & Outcome & $\begin{array}{l}\text { Median } \\
\text { PFOS/PFOA conc. } \\
\text { (ng/ml) }\end{array}$ & Results \\
\hline $\begin{array}{l}\text { Lin, } 2009 \\
\text { (Lin et al., 2009) }\end{array}$ & $\begin{array}{l}\text { USA/ } \\
\text { NHANES }\end{array}$ & $\begin{array}{l}\text { 474adolesve } \\
\text { nts }+696 \\
\text { adults }\end{array}$ & $\begin{array}{l}\text { cross- } \\
\text { sectional }\end{array}$ & $\begin{array}{l}1999- \\
2000 \text { and } \\
2003- \\
2004\end{array}$ & 1,443 & $\begin{array}{l}\text { PFOA. PFNA. } \\
\text { PFOS. PFHXS }\end{array}$ & $\begin{array}{l}\text { Glucose homeosta- } \\
\text { tis. metabolic } \\
\text { syndrome }\end{array}$ & $\begin{array}{l}\text { PFOS: } 24.3 \text { PFOA: } \\
4.3\end{array}$ & $\begin{array}{l}\text { Increased serum PFNA conc. associated } \\
\text { with hyperglycemia; serum HDL choles- } \\
\text { terol; inversely with prevalence of meta- } \\
\text { bolic syndrome }\end{array}$ \\
\hline $\begin{array}{l}\text { Nelson, } 2010 \\
\text { (Nelson et al., 2010) }\end{array}$ & $\begin{array}{l}\text { USA/ } \\
\text { NHANES }\end{array}$ & $\geq 12$ years & $\begin{array}{l}\text { cross- } \\
\text { sectional }\end{array}$ & $\begin{array}{l}2003- \\
2004\end{array}$ & 2,094 & $\begin{array}{l}\text { PFOA. PFNA. } \\
\text { PFOS. PFHXS }\end{array}$ & $\begin{array}{l}\text { Lipid and weight } \\
\text { outcomes }\end{array}$ & $\begin{array}{l}\text { PFOS:19.9 PFOA: } \\
3.8\end{array}$ & $\begin{array}{l}\text { A positive association between concentra- } \\
\text { tions of PFOS, PFOA, and PFNA and total } \\
\text { and non-high-density cholesterol }\end{array}$ \\
\hline \multicolumn{10}{|c|}{ Cardiovascular diseases } \\
\hline $\begin{array}{l}\text { Shankar, } 2012 \\
\text { (Shankar } \\
\text { et al., 2012) }\end{array}$ & $\begin{array}{l}\text { USA/ } \\
\text { NHANES }\end{array}$ & $\begin{array}{l}\text { Men and } \\
\text { women } \\
\text { above } 40 \\
\text { years }\end{array}$ & $\begin{array}{l}\text { Cross- } \\
\text { sectional }\end{array}$ & $\begin{array}{l}1999- \\
2000 \text { and } \\
2003- \\
2004\end{array}$ & $\begin{array}{l}1,216 \\
(51.2 \% \\
\text { wom- } \\
\text { en) }\end{array}$ & PFOA & $\begin{array}{l}\text { Cardiovascular } \\
\text { disease and } \\
\text { peripherial arterial } \\
\text { disease }\end{array}$ & Not reported & $\begin{array}{l}\text { Exposure to PFOA is associated with } \\
\text { cardiovascular disease and peripherial } \\
\text { arterial disease, independent of tradi- } \\
\text { tional cardiovascular risk factors }\end{array}$ \\
\hline
\end{tabular}

BW: Birth weight; GSA: Gestational age.

Environmental effects of per-and polyfluorinated substances. 


\section{Environmental effects of per- and polyfluorinated substances}

The general observed trend of PFC toxicity is the linear relationship found between increasing chain-length and decreasing $\mathrm{EC}_{50}$ (Hoke et al., 2012; Latała et al., 2009; Mitchell et al., 2011; Mulkiewicz et al., 2007; Nobels et al., 2010). This in combination with the longer half-lives and elimination rate of the longer chain PFCs should be recognised as a great health and environmental concern (Mulkiewicz et al., 2007). It has been shown that PFCs may affect the thyroid system (Weiss et al., 2009), influence the calcium homeostasis, protein kinase $C$, synaptic plasticity, cellular differentiation, induce neurobehavioral effects and induce peroxisome proliferation (Ishibashi et al., 2008; Mariussen, 2012). In vitro assessment of environmental fate and ecotoxicologial effects of individual PFCs in some cases demonstrate that their current environmental levels do not pose a threat to ecosystems (O'Brien et al., 2009). On the other hand, in the environment, exposure is rarely limited to one PFC, but to a mixture of various PFCs and other environmental pollutants. Toxic effects may occur as a result of interactions between hazardous chemicals and co-exposure may cause additive or synergistic effects (Eriksen et al., 2010). Latała et al. (2009) therefore suggest that future studies of PFCs ecotoxicity should mainly focus on the effects of mixtures of PFCs and their derivatives. Another factor that should be considered when PFC levels in wildlife are evaluated is the substantial differences in PFC concentrations found among life history stages. In a study on porpoises, the highest concentrations of PFCs were found in neonates, suckling juveniles and lactating females which is of concern as PFCs are known to cause toxic effects on the development of the central nervous system and reproductive organs (Galatius et al., 2011).

Some mechanistic studies on PFC toxicity have revealed that there are species specific differences. However, this was not the outcome when PFOS, PFOSA, PFHxA and PFBS were studied (Hu et al., 2002) in both a rat liver epithelial cell line (WB-F344) and a dolphin kidney epithelial cell line (CDK) for GJIC interfering effects. Gap junctional intercel- 
lular communication (GJIC) is the major pathway of intercellular signal transduction, and is therefore important for normal cell growth and function. Inhibition of GJIC was found in a dose-dependent manner for all compounds except for PFBS and the inhibitory effects were neither species- nor tissue specific. A Specific Absorption Rate (SAR) was also established among the four compounds tested, and the effect was determined by the length of the fluorinated tail and not by the functional groups. This is contradicted by a study made by Hagenaars et al. (2011a), comparing the potential effects of the four different PFCs; PFOS, PFBS, PFOA and PFBA on embryonic development in zebrafish (Danio rerio). The authors conclude that sulfonated and carboxylated PFCs act by different processes and that the exact mechanisms of the potential effects of PFOS, PFOA and PFBS on endothelial cells or vasoactive substances of the heart will need further investigation.

In order to evaluate the potential reproductive effects of PFCs, a study was conducted on the toxic effects of the four PFCs; PFOA, PFNA, PFOS and FC-807 (perfluoro alkyl phosphate) on zebrafish embryos. Oedemas and spine malformations occurred throughout the duration of the study and all the tested PFCs caused lethality in a concentrationdependent fashion. Based on the $\mathrm{LC}_{50}$ values the toxic potency was in the order of PFOS $>$ FC807 $>$ PFNA $>$ PFOA. Although all four compounds caused malformations, FC-807, with the larger ester molecule, caused more yolk-sac and pericardial-sac oedemas than the other PFCs. The authors therefore propose that $\mathrm{FC}-807$ might more easily disturb the water barrier around the embryos and disturb heart functions, causing oedemas (Zheng et al., 2012).

\subsection{Perfluoro carboxylates (PFCAs)}

PFCA toxicity has been tested in different organisms. Blue-green algae and cyanobacteria were shown to be very sensitive to PFCAs (Latała et al., 2009). E. coli bacteria were exposed to selected PFCs which resulted in clear indications of markers for oxidative stress and DNA damage with the most severe DNA damage observed after exposure to PFDA, PFUnDA and PFDoDA (Nobels et al., 2010). The structure-activity results from the $E$. coli study (Nobels et al., 2010) indicated that the carboxylic acids with long carbon tails induced several stress genes. Other studies have made similar findings; ROS and DNA damage was observed in HepG2 cells after exposure to PFCAs (Eriksen et al., 2010), cytotoxicity was found against primary rat testicular cells, where PFNA attributed as most DNA damag- 
ing (Lindeman et al., 2012). In vitro cytotoxicity of PFCAs was determined in human colon carcinoma (HCT116) cells (Kleszczyński et al., 2007; Kleszczyński and Składanowski, 2009) and treatment with PFCAs caused cell apoptosis. It seems that the PFCAs are not acutely toxic, but the cell viability inhibition is intensified after longer exposure. The estimated $\mathrm{EC}_{50}$ values decreased with elongation of the fluorocarbon chain (PFHxA > PFHpA $>$ PFOA $>$ PFNA $>$ PFDA $>$ PFDoA $>$ PFTeDA), although, chain lengths above $\mathrm{C} 16$ and $\mathrm{C} 18$ did not increase the effect. PFCA effects on hatching success of birds was investigated by O'Brien et al. (2009) and in their results, only PFUDA caused statistically significant increases in gene transcription at the highest dose applied $(10 \mu \mathrm{g} / \mathrm{g})$.

PFCAs are also potent inducers of the oestrogen-responsive biomarker protein vitellogenin (Vtg). This effect was investigated in vivo in rainbow trout by Benninghoff et al. (2011). They found that all PFCAs tested (PFOA, PFNA, PFDA and PFUnDA) bound weakly to trout liver ER with $\mathrm{IC}_{50}$ values of $15.2-289 \mathrm{mM}$, whereas, 8 to 10 fluorinated carbons and a $\mathrm{COOH}$ end group were optimal for Vtg induction. The same authors suggest that multiple PFCAs can promote hepatic tumorigenesis in trout in a manner similar to that of $17 \beta$-estradiol (Benninghoff et al., 2012).

\subsection{Perfluoroalkyl sulfonates (PFSAs)}

The perfluoroalkyl sulfonates contain a sulfonate group instead of a carboxyl group, but as mentioned earlier, there still seems to be no clear understanding of the relative importance of chain length and functional groups for the toxic effects of PFCs.

Some of the bigger PFC producers, such as $3 \mathrm{M}$, have already phased out the production of PFOS (C8) and replaced it with the production of PFBS (perfluorobutane sulfonate, C4) as an alternative. Due to its shorter chain length it is expected to be less bioaccumulative. As a result, the production volumes of PFBS have expanded during the last decade and PFBS has already been detected in environmental samples. The general lack of information on its toxicological mode of action and the potential hazard it may pose against ecological species, has therefore directed a great deal of focus on PFBS during the last years (Hagenaars et al., 2011b). According to the obtained ecotoxicological information on PFBS, its direct toxicity seems to be relatively low. Flow cytometric measurements on some membrane systems of the freshwater alga species Scenedesmus obliquus revealed that PFBS did not inhibit algal growth within the test concentration ranges (Liu et al., 2008). PFBS was exam- 
ined by Rosal et al. (2010) against the marine bacterium Vibrio fischeri, the cyanobacterial recombinant strain Anabaena CPB4337 and the algae Pseudokirchneriella subcapitata and the toxicity against all organisms tested was very low (EC50 > 8,000 ml/L). In the study mentioned above on effects of PFBS on embryonic development in zebrafish (Danio rerio), it was not possible to determine its LC50 value due to the low mortality rate of the compound even at high exposure concentrations (500 and $3,000 \mathrm{mg} / \mathrm{L}$ ). The results however, demonstrated significantly altered heart rates in the embryos as well as malformations of their heads after exposure to PFBS (Hagenaars et al., 2011b). In juvenile mallards and northern bobwhite quail, PFBS was found to affect the body weight gains of quail exposed to 5,620 or 10,000 mg PFBS/ kg feed, which were statistically less than that of unexposed controls (Newsted et al., 2008).

What appears to be of more concern regarding the short-chained PFCs is their elicited transcriptional responses. Long-chained PFCs bind more strongly to extracellular proteins than short-chained PFCs, which may render their availability for uptake into cells. Hence, short-chained PFCs may be more bioavailable and may cause more genetic and neural damage (Vongphachan et al., 2011).

Slotkin et al. (2008) used PC12 cells, (a standard in vitro model for neuronal development) to characterise essential features of the developmental neurotoxicity of PFBS. PFBS demonstrated a unique effect on differentiation of both dopamine (DA) and acetylcholine (ACh) neurotransmitter phenotypes; displaying a concentration-dependent suppression in both the expression of TH (Tyrosine Hydroxylase) and ChAT (Choline AcetylTransferase). PFBS did not evoke any effect on DNA synthesis, although it did produce significant cell enlargement due to an increase in the total protein/DNA ratio (Slotkin et al., 2008).

The short-chained PFCs, PFBS and PFHxS were shown to significantly alter the messenger RNA (mRNA) of TH-responsive genes in primary cultures of avian (chicken embryonic and herring gull embryonic) neuronal cells. Exposure to these compounds resulted in e.g. induction of D3 mRNA, RC3 mRNA and down-regulation of TTR mRNA (Vongphachan et al., 2011). These effects could affect e.g. TH-dependent processes as well as changes in RC3 expression could have consequences in synaptic plasticity, associative learning and memory (Iniguez et al., 1993; Iniguez et al., 1996; Vongphachan et al., 2011). Likewise, an earlier in vitro study on CEH (Chicken Embryo Hepatocyte) cultures exposed to PFHxS, PFHpS and PFDS demonstrated alterations of transcriptional responses. The shorterchained PFHxS and PFHpS induced CYP1A4 or CYP1A5 mRNA, while the longer-chained PFDS repressed CYP1A4 mRNA (Hickey et al., 2009). 
These reported significant effects of PFHxS treatment on messenger RNA (mRNA) levels of thyroid hormone (TH)-responsive genes in chicken embryonic neuronal cells initiated the determination of in ovo effects of PFHxS exposure (maximum dose $=38,000 \mathrm{ng} / \mathrm{g}$ egg). The previous in vitro results were successfully validated, since plasma TH levels of chicken embryos were reduced in a concentration-dependent manner following PFHxS exposure. In addition, pipping success was significantly reduced, the tarsus length and embryo mass decreased and D2 and D3 and cytochrome P450 3A37 mRNA levels were induced in the liver tissue, whereas D2, RC3 and octamer motif binding factor 1 mRNA levels were up-regulated in cerebral cortex. PFHxS accumulation could be seen in the three tissue compartments analysed: yolk sac > liver > cerebral cortex (Cassone et al., 2012).

In an in vivo study (Nøst et al., 2012), plasma concentrations of a wide range of halogenated organic contaminants, including PFHxS and PFHpS, and their correlations with circulating thyroid hormones (TH) in developing Arctic seabirds was assessed. Plasma from chicks of blacklegged kittiwake (Rissa tridactyla) and northern fulmar (Fulmarus glacialis) was taken and analysed for thyroid hormones. A positive association was found between total thyroxin (TT4) and PFHpS in both species and PFHxS was negatively correlated to the TT3:FT3 ratio. Since the disruption of TH homeostasis may cause developmental effects in young birds, the correlations between the relatively low plasma levels of PFCs and THs found in the study may be of concern on the health related effects of these compounds in seabird fledglings (Nøst et al., 2012).

The C10 PFSA, PFDS, seems to be less industrially utilised and has accordingly attained less attention, which is reflected by its appearance in the scientific literature. But since Hickey et al. (2009) identified the responsiveness of genes exposed to PFDS in in vitro cultured chicken embryonic hepatocytes (CEH) the in ovo toxicity of PFDS was assessed by injection into white leghorn chicken (Gallus gallus domesticus) eggs (O'Brien et al., 2009). The compound was detected in livers of the chicken embryos, at levels in agreement with concentrations (up to $10 \mu \mathrm{g} / \mathrm{g}$ ) injected, indicating that PFDS was efficiently taken up by the developing embryos. Despite this, transcriptional activity for CYP1A4, CYP1A5, CYP4B1 or L-FABP mRNA in the chicken embryo liver tissue was not significant altered and the pipping success was not affected (O’Brien et al., 2009). 


\section{$9.3 \quad$ FTOHs}

The PFOS and PFOA use has been exchanged for shorter chain PFCs such as the fluorotelomer alcohols. Little data on the distribution and environmental fate of FTOH makes it difficult to assess the environmental risk. Available studies of their biological and ecotoxicological assessment have demonstrated the need to monitor their environmental distribution and further investigate their effects on the biota, especially for longterm exposure of environmental relevant concentrations. FTOHs have been detected in the aquatic environment and their biotic and abiotic degradation lead to a range of products, including PFCAs of various chain lengths, causing secondary pollution. PFOA and PFNA have been confirmed as metabolites of 8:2 FTOH (Ishibashi et al., 2008; Martin et al., 2005), and since PFCAs, particularly PFOA, have been reported to cause liver cancer, pancreatic tumour, and Leydig cell tumour, FTOHs might indirectly induce tumours via PFCAs (Oda et al., 2007).

As other PFCs, FTOHs have been characterised as xenoestrogens in vitro and owe their oestrogen-like effects to their structural and chemical similarities to other xenoestrogenic compounds. Fluorotelomer alcohols have been shown to exert estrogenic activity in MCF-7 cells, in a yeast two-hybrid assay and in aquatic organisms where 6:2 FTOH has been characterised as a stronger xenoestrogen than 8:2 FTOH (Ishibashi et al., 2008; Maras et al., 2006; Vanparys et al., 2006; Wang et al., 2012). According to Ishibashi et al. (2007) treatment with 6:2 FTOH, 8:2 FTOH and NFDH (nonadecafluoro-1-decanol) show a dose-dependent interaction with the human estrogen receptor (hER), and rank the estrogenic effects for hER $\alpha$ and hER $\beta$ in the descending order of $17 \beta$-estradiol >>> 6:2 FTOH $>$ NFDH $>8: 2$ FTOH. Waterborne exposure of both 6:2 and 8:2 FTOH alter the plasma levels of testosterone and estradiol and 8:2 FTOH adversely impair reproductive success in the offspring of zebrafish (Liu et al., 2010a; Liu et al., 2009). In addition, it has been suggested that 8:2 FTOH has the potential to suppress stereoidgenesis (Liu et al., 2010b).

The fluorotelomer alcohols have been ecotoxocologically assessed through their growth impairment potential. Wang et al. (2010) suggested that 4:2 and 6:2 FTOH might cause apoptosis, while, Oda et al. (2007) suggested that they are unlikely mutagens. The fluorotelomer alcohols 8:2 FTOH and 10:2 FTOH were shown to be rapidly metabolised by rainbow trout to the fluorotelomer acids (8:2 FTCA, 10:2 FTCA) and the unsaturated acids (8:2 FTUCA, 10:2 FTUCA), respectively (Brandsma et al., 2011). Studies have found that these transformation products are more bioaccumulative and more acutely toxic to aquatic organisms than their 
precursors (Brandsma et al., 2011; Hoke et al., 2012; Mitchell et al., 2011). Both Hoke et al. (2012) and Mitchell et al. (2011) suggest, however, that these fluorinated acids pose little or negligible risk to aquatic biota, since the available environmental concentrations are still well under the toxicity thresholds.

\subsection{Other fluorinated compounds of interest}

Polyfluorinated iodine alkanes (PFIs) are important intermediates in the synthesis of organic fluoride products. Recently, they have been detected in fluoropolymers as residual raw materials, as well as in the ambient environment. Wang et al. (2012) studied for the first time the estrogenic activity of PFIs, fluorinated iodine alkanes (FIAs), fluorinated telomer iodides (FTIs), and fluorinated di-iodine alkanes (FDIAs) in MCF-7 cells. They concluded that some PFIs could act on ERs and potentially cause detrimental effects on reproductive and developmental systems (Wang et al., 2012).

Semi-fluorinated emulsifiers derived from the dimorpholinophosphate polar head group $\mathrm{CnF} 2 \mathrm{n}+1(\mathrm{CH} 2) \mathrm{mOP}(0)[\mathrm{N}(\mathrm{CH} 2 \mathrm{CH} 2) 2 \mathrm{O}] 2$ (FnHmDMP) allow for the preparation of stable water-in-fluorocarbon emulsions. These emulsions are being investigated as delivery systems of drugs into the lung, either by systemic or local administration. The cytotoxicity of a series of FnHmDMP was evaluated by Courrier et al. (2003). FnHmDMP compounds with the longest fluorinated chain length or total chain length ratio, i.e. F8H11DMP and F10H11DMP were shown to be the least toxic. Moreover, emulsions stabilised with these amphiphiles were found to be non-cytotoxic, or less cytotoxic than solutions of the same amphiphiles in fluorocarbons (Courrier et al., 2003).

Fluorotelomer unsaturated aldehydes (FTUALs) and acids (FTUCAs) are intermediate metabolites that form from the degradation of FTOHs. Their toxicity potential is not yet defined and may be more significant compared to PFCAs, but studies have shown that they form adducts with glutathione (GSH). Results presented by Rand and Mabury (2012) indicate that the $\alpha, \beta$-unstaurated aldehydes react most comparatively with GSH and that the reaction is possibly influenced by the length of the fluorinated tail. They also suggest that given the low EC50 values measured for 6:2 FTUAL and 8:2 FTUAL, these compounds may exert cytotoxic influences on biological nucleophiles present in proteins as well as nucleic acids. 



\section{Discussion}

There are considerable data gaps on the content of specific PFCs in commercial products used on the Nordic market. Some of these PFCs exhibit hazardous characteristics and therefore it is of very high concern to facilitate access to specific PFC substance information from industrial actors on the market either on a voluntary basis or if this is not possible by legal means. The current legal tools such as the EC Regulation 1272/2008 (CLP) and the EC Regulation 1907/2006 (REACH) are currently not sufficient to provide that kind of specific substance information although the information exists. For publicly available MSDS there is no legal incentive for a company to provide specific substance data and when provided to the authority this information is legally classified as confidential with no access to the public. Concerning PFCs in articles it is not possible to achieve specific PFC substance information according to REACH unless they are identified as Substances of Very High Concern (SVHC). Then there is a legal possibility to access downstream information. However, this is only possible if the concentration of the PFC (then as an SVHC) exceeds $0,1 \%$ by weight of the article in question. Since many PFCs are added in much lower concentrations in products, the SVHC approach to PFCs may be ineffective from a legal perspective. It is important to mention that there are small opportunities to get production data on specific PFCs in articles since almost all production occurs outside the EU.

There are few studies on PFCs in the Nordic environment. Therefore there is an urgent need for new data on PFCs, especially for PFCs other than PFOA and PFOS, regarding their environmental occurrence. This is necessary if we want to get a better and more complete picture of the PFC levels in different Nordic environmental compartments. This includes more in-depth knowledge of spatial and temporal distribution, and clear temporal trends.

Modeling and field monitoring are essential prerequisites for detailed environmental fate studies of PFCs. In many cases these studies are hindered by the lack of reliable (or in some cases total lack of) physicalchemical properties for many fluorinated compounds. Furthermore, there is still a lack of analytical reference standards of PFCs but lately there is an increased access to new and better reference standard sub- 
stances on the market which are necessary for these kinds of environmental studies. Further resources for in depth research are thus needed.

There are few studies on biomonitoring of PFCs other than PFOA and PFOS. Therefore there is a great need for further studies. This is especially true for those with shorter carbon chains and their corresponding precursors in human/maternal blood and cord blood. There is also a need to explore the real pre-term and post-term exposure of the fetus and newborn child. For some less known PFCs such as PFAL (perflouroaldehyde), FTS (fluorotelomer sulfonates), PAP/di-PAP and FTMAPs (fluorotelomer mercaptoalkyl phosphate diester) there are no studies at all carried out and consequently no data is available. Also in this case further in-depth research is needed. Further studies concerning PFCs' impact on maternity and immunology are called for since only inconsistent data exist. 


\section{Conclusions}

As a result of the mapping study, stage 1 of this project, carried out on more than 50 actors on the Nordic market that trade with PFC products it was concluded that there are considerable information gaps for most of the PFC chemicals regarding the exact chemical composition in commercial products, their quantities produced and uses on the Nordic market. These gaps may be a combination of lack of knowledge and/or trade secrets from the actors on the Nordic market.

In parallel with the mapping of the Nordic market a net list of specific PFCs that may be used on the market was produced. This net list was extracted from three public lists, namely one list from OECD, the REACH preregistration database, and the Nordic SPIN database. Since neither of these databases contains complete information on the market use of PFCs, the net list is necessarily incomplete and there may be other PFCs used on the Nordic market in addition to those found in the net list.

There exists only a few scientific reports on PFCs in the Nordic environment other than PFOA and PFOS that cover both biotic and abiotic samples. Regarding PFCAs, most studies report results for PFOA, PFHxA and PFNA. However other PFCA substances (C10-C13) have also been detected in a few studies. For PFSAs, PFOS and PFHxS are the most studied compounds. Observations reported in the few studies available report that the concentrations in the Nordic environment and the Arctic are much lower compared to other countries especially when compared with central European countries with high GDPs, which is to be expected as populations are smaller and there is less industry in the Nordic countries. However these substances have also been found in the Arctic, far from any sources, which shows that these substances are global contaminants.

Publications that report human biomonitoring data of PFCs (PFCAs and PFSAs) for the Nordic countries during the period from 1992 to 2010 are available. Most and most recent data are reported from Norway and Sweden, whereas fewer exist from Denmark. No human data were found for Iceland and Finland. Results from these studies report that since 2002 decreasing trends have been observed for PFOA and PFOS but not for other PFCAs and PFSAs. In Sweden, for instance, it was found that perfluorinated sulfonates with shorter carbon chains $(\leq 6)$ currently show an increasing trend. 
Only a few studies on PFSAs and PFCAs in amniotic fluids have been published but all show low levels that are 10-20 folds below the levels in the corresponding serum. Nordic studies show that the PFSAs and PFCAs can be transferred to human breast milk with a concentration range of $1-2 \%$ and $3-4 \%$, respectively of the serum concentration. For other PFCs such as PFAL (perflouroaldehyde), FTS (fluorotelomer sulfonates), PAP/di-PAP and FTMAPs (fluorotelomer mercaptoalkyl phosphate diester) no studies have been carried out.

Animal studies on toxicity show that PFCAs and PFSAs affect the development, reproduction and immune system negatively by decreasing body weight, inducing hepatoxicity, affecting the endocrine system including the sex hormone and thyroid hormone system. Hepatocytic hypertrophy effect in laboratory animals were reported for PFOS, PFHxS, PFBS, PFDA, PFNA, PFOA, PFHpA, PFHxA, and PFBA and is likely associated with induced peroxisome proliferation.

Early pregnancy loss was observed in animal studies with PFOA or PFBA exposure but only at very high doses, and the etiology of this effect is not clear. No fetal toxicity was observed after gestational exposure to PFBA or PFDA. Compared to long-chain PFAAs $(\geq C 8)$, the short-chain chemicals are much less toxic to the developing animal, in part due to their faster rate of clearance. A similar lack of reproductive and developmental toxicity has been reported for PFHxA, PFBS and PFHxS.

Adverse immunological outcomes have been reported from exposure to PFOS, PFHxS, PFOA and PFNA. Alterations of thyroid hormones and sex steroid hormones (endocrine disruption) have been shown after exposure to primarily PFOS and PFOA, although PFDA-induced reductions of thyroid hormones have also been reported. PFDoA has recently been shown to decrease testosterone synthesis in male rats and to decrease serum estradiol and gene expression of estrogen receptors in the female rats, possibly through oxidative stress pathways.

The overall observations on liver parameters such as lipid profile, the reproductive (e.g. menopause), the thyroid hormone system, and the risk of ADHD (PFHxS) were observed as a combined effect of PFCAs and PFSAs. Follow-up evaluations of infants and children in the Danish National Birth Cohort indicated no associations between prenatal exposure to PFAAs and risk of infectious diseases, normal developmental milestones, and behavioral and motor coordination problems. Whereas a study on the Faroe Islands birth cohort showed that PFC levels inversely correlated to the vaccination response at age 5 .

A linear relationship between increasing PFC chain-length and decreasing $\mathrm{EC}_{50}$ has been observed. This in combination with the longer 
half-lives and elimination rate of the longer chain PFCs should be recognised as a great health and environmental concern. In the environment, exposure is rarely limited to one PFC, but to a mixture of various PFCs and other environmental pollutants. Toxic effects may occur as a result of interactions between hazardous chemicals and co-exposure may cause additive or synergistic effects. Future studies of PFCs ecotoxicity should focus on the effects of mixtures of PFCs and their derivatives. 



\section{References}

\section{Mapping of use of per- and polyfluorinated substances on the Nordic market}

Lists of PFOS, PFAS, PFOA, PFCA, Related Compounds and Chemicals that may degrade to PFCA (as revised in 2007). Organisation for Economic Cooperation and Development, 21 August 2007. ENV/JM/MONO(2006)15:

- http://echa.europa.eu/information-on-chemicals/ pre-registered-substances

- http://www.spin2000.net/

Daae et al. 2009. Kjemisk eksponering og effekter på luftveiene blant profesjonelle skismørere. Statens Arbeidsmiljøinstitut.

Kissa, E., Fluorinated surfactants and repellents. 2001. Surfactant Science Series, Marcel Dekker, New York, NY Vol 97, (Fluorinated Surfactants and Repellents (2nd Edition)), 1-615.

Knepper, T.P., Lange, F.T., (eds) 2011. Polyfluorinated Chemicals and Transformation Products. Handbook of Environmental Chemistry, Springer Verlag, Vol 17, ISBN 978-3-642-21871-2 Poulsen et al. 2011. Substitution of PFOS for use in non decorative hard chrome plating. Environmental Project No. 1371 2011, Danish Ministry of the Environment.

Schultze P-E, Norin H. Fluorinated pollutants in all-weather clothing, Friends of the Earth Norway - Report 2/2006, Swedish Society for Nature Conservation http://www.nikwax.com/cmsdata/Downloads/pr/Children's_Clothing_Report2.pdf

Poulsen et al. 2005. More environmentally friendly alternatives to PFOS-compounds and PFOA. Environmental project No. 1013 2005, Danish Ministry of the Environment.

UNEP/POPS/POPRC.6/13/Add.3/Rev.1

UNEP/POPS/POPRC.8/INF/17

\section{Emissions to and occurrence of PFCs into the environment}

Bakke, T., Fjeld, E., Skaare, B., Berge. J.A., Green, N., Ruus, A., Schlabach, M., and Botnen, H. 2007. Kartlegging av metaller og utvalgte nye organiske miljøgifter 2006. Krom, arsen, perfluoralkylstoffer, dikloretan, klorbenzener, pentaklorfenol, HCBD og DEHP. SFT TA2284/2007. NIVA report 5464-2007.105s.

Barber, J.L., Berger, U., Chaemfa, C., Huber, S., Jahnke, A., Temme, C., Jones, K.C. 2007. Analysis of per- and polyfluorinated alkyl substances in air samples from Northwest Europe. Journal of Environmental Monitoring, 9 (6), 530-541.

Björklund, J.A., Thuresson, K., De Wit, C.A. 2009. Perfluoroalkyl compounds (PFCs) in indoor dust: Concentrations, human exposure estimates, and sources. Environmental Science and Technology, 43 (7), 2276-2281. 
Bossi, R., Riget, F.F., Dietz, R. 2005. Temporal and spatial trends of perfluorinated compounds in ringed seal (Phoca hispida) from Greenland. Environmental Science and Technology, 39 (19), 7416-7422.

Bossi, R., Riget, F.F., Dietz, R., Sonne, C., Fauser, P., Dam, M., Vorkamp, K. 2005. Preliminary screening of perfluorooctane sulfonate (PFOS) and other fluorochemicals in fish, birds and marine mammals from Greenland and the Faroe Islands. Environmental Pollution, 136 (2), 323-329.

Bossi, R., Strand, J., Sortkjær, O., Larsen, M.M. 2008. Perfluoroalkyl compounds in Danish wastewater treatment plants and aquatic environments. Environment International, 34 (4), 443-450.

Butt, C.M., Berger, U., Bossi, R., Tomy, G.T. 2010. Levels and trends of poly- and perfluorinated compounds in the arctic environment. Science of the Total Environment, 408 (15), 2936-2965.

Dinglasan-Panlilio, M. J. A., Mabury, S. A. 2006. Significant Residual Fluorinated Alcohols Present in Various Fluorinated Materials. Environmental Science and Technology. 40, 1447-1453.

Fjeld, E., Schlabach, M., Berge J.A., Green, N., Eggen, T., Snilsberg, P., Vogelsang, C., Rognerud, S., Kjellberg, G., Enge, E.K., Dye, C.A., Gundersen, H. 2005. Kartlegging av utvalgte nye organiske miljøgifter 2004. Bromerte flammehemmere, perfluoralkylstoffer, irgarol, diuron, BHT og dicofol, NIVA rapport nr 5011-2005.

Galatius, A., Dietz, R., Rigét, F.F., Sonne, C., Kinze, C.C., Lockyer, C., Bossi, R. 2011. Temporal and life history related trends of perfluorochemicals in harbor porpoises from the Danish North Sea. Marine Pollution Bulletin, 62 (7), 1476-1483.

Haug, L.S., Huber, S., Becher, G., Thomsen, C. 2011b. Characterisation of human exposure pathways to perfluorinated compounds - Comparing exposure estimates with biomarkers of exposure. Environment International, 37 (4), 687-693.

Haug, L.S., Huber, S., Schlabach, M., Becher, G., Thomsen, C. 2011. Investigation on perand polyfluorinated compounds in paired samples of house dust and indoor air from Norwegian homes. Environmental Science and Technology, 45 (19), 7991-7998.

Haukås, M., Berger, U., Hop, H., Gulliksen, B., Gabrielsen, G.W. 2007. Bioaccumulation of per- and polyfluorinated alkyl substances (PFAS) in selected species from the Barents Sea food web. Environmental Pollution, 148 (1), 360-371.

Huber, S., Haug, L.S., Schlabach, M. 2011. Per- and polyfluorinated compounds in house dust and indoor air from northern Norway - A pilot study. Chemosphere, 84 (11), 1686-1693.

Jahnke, A., Huber, S., Temme, C., Kylin, H., Berger, U. 2007. Development and application of a simplified sampling method for volatile polyfluorinated alkyl substances in indoor and environmental air. Journal of Chromatography A, 1164 (1-2).

Kallenborn, R., Berger, U., Järnberg, U., Dam, M., Glesne, O., Hedlund, B. 2004. Perfluorinated alkylated substances (PFAS) in the Nordic environment. Nordic Council of Ministers.

Liu, J., Li, J., Liu, Y., Chan, H.M., Zhao, Y., Cai Z., Wu Y. 2011. Comparison on gestation and lactation exposure of perfluorinated compounds for newborns. Environ Int 37(7): 1206-1212.

Löfstrand, K., Jörundsdóttir, H., Tomy, G., Svavarsson, J., Weihe, P., Nygård, T., Bergman, Å. 2008. Spatial trends of polyfluorinated compounds in guillemot (Uria aalge) eggs from North-Western Europe. Chemosphere, 72 (10), 1475-1480.

Miljeteig, C., Strøm, H., Gavrilo, M.V., Volkov, A., Jenssen, B.M., Gabrielsen, G.W. 2009. High levels of contaminants in ivory gull Pagophila eburnea eggs from the Russian and Norwegian arctic. Environmental Science and Technology, 43 (14), 5521-5528. 
Report number TA-2354/2007. Norwegian Pollution Control Authority, Survey of National Sources, 2007.

Report number TA-2367/2008. Green, N., Schlabach, M., Bakke, T., Brevik, E.M., Dye, C., Herzke, D. Screening of selected metals and new organic contaminants 2007. Norwegian Pollution Control Authority, Oslo, Norway.

Report number TA-2444/2008. Green, N., Schlabach, M., Bakke, T., Brevik, E.M., Dye, C., Herzke, D. 2008. Screening of polyfluorinated organic compounds at four fire training facilities in Norway. Norwegian Pollution Control Authority, Oslo, Norway. Norwegian Pollution Control Authority, Oslo, Norway.

Report number TA-3005/2012. Andersen, S., Gudbrandsen, M., Haugstad, K., Hartnik, T. 2012. Noen miljøskadelige stoffer i avløpsslam - forekomst og miljørisiko. Climate and Pollution Agency, Oslo, Norway.

Smithwick, M., Mabury, S.A., Solomon, K.R., Sonne, C., Martin, J.W., Born, E.W., Dietz, R., Derocher, A.E., Letcher, R.J., Evans, T.J., Gabrielsen, G.W., Nagy, J., Stirling, I., Taylor, M.K., Muir, D.C.G. 2005. Circumpolar study of perfluoroalkyl contaminants in polar bears (Ursus maritimus). Environmental Science and Technology, 39 (15), 5517-5523.

Telomer Research Program 2002. Telomer Research Program Update, Presented to the USEPA OPPT, November 25, 2002; U.S. Environmental Protection Agency public docket AR226-1141.

Theobald, N., Gerwinski, W., Caliebe, C., Haarich, M. 2007. Development and validation of a method for the determination of polyfluorinated organic substances in sea water, sediments and biota. Occurrence of these compounds in the North and Baltic Seas, Umweltbundesamt.

Verreault, J., Berger, U., Gabrielsen, G.W. 2007. Trends of perfluorinated alkyl substances in herring gull eggs from two coastal colonies in northern Norway: 19832003. Environmental Science and Technology, 41 (19), 6671-6677.

\section{Modelling of emissions to the environment}

Bennett, D.H., McKone, T.E., Matthies, M., Kastenberg, W.E., 1998. General formulation of characteristic travel distance for semivolatile organic chemicals in a multimedia environment. Environmental Science \& Technology 32 (24), 4023-4030.

Beyer, A., Mackay, D., Matthies, M., Wania, F., Webster, E., 2000. Assessing long-range transport potential of persistent organic pollutants. Environmental Science \& Technology 34 (4), 699-703.

Breivik, K., Sweetman, A., Pacyna, J.M., Jones, K.C., 2007. Towards a global historical emission inventory for selected PCB congeners - A mass balance approach. 3 . An update. Science of the Total Environment 377 (2-3), 296-307.

Breivik, K., Wania, F., Muir, D.C.G., Alaee, M., Backus, S., Pacepavicius, G., 2006. Empirical and modeling evidence of the long-range atmospheric transport of decabromodiphenyl ether. Environmental Science \& Technology 40 (15), 4612-4618.

Pistocchi, A., Loos, R., 2009. A map of European emissions and concentrations of PFOS and PFOA. Environmental Science and Technology 43 (24), 9237-9244.

Wania, F., 2006. Potential of degradable organic chemicals for absolute and relative enrichment in the arctic. Environmental Science \& Technology 40 (2), 569-577.

Wania, F., Mackay, D., 1996. Tracking the distribution of persistent organic pollutants. Environmental Science \& Technology 30 (9), A390-A396. 


\section{Food and drinking water}

Clarke, D.B., Bailey, V.A., Routledge, A., Lloyd, A.S., Hird, S., Mortimer, D. N., Gem, E.M. 2010. Dietary intake estimate for perfluorooctanesulphonic acid (PFOS) and other perfluorocompounds (PFCs) in UK retail foods following determination using standard addition LC-MS/MS. Food Additives and Contaminants, 27, 4, 530-545.

Falandysz, J., Taniyasu, S., Gulkowska, A., Yamashita, N. and Schulte-Oehlmann, U. 2006. Is fish a major source of fluorinated surfactants and repellents in human living on the Baltic coast. Environ. Sci. Technol., 40, 748-751.

Fromme, H., Schlummer, M., Möller, A., Gruber, L., Wolz, G., Ungewiss, J., Böhmer, S., Dekant, W., Mayer, R., Liebl, B. and Twardella, D. 2007. Exposure of an adult population to perfluorinated substances using dublicate diet portions and biomonitering data. Environ. Sci. Technol., 41, 7928-7933.

Granby, K., Technical University of Denmark. 2012. PFCs in Danish fish and animal food (not published data).

Haug, L.S., Salihovic, S., Jogsten, I.E., Thomsen, C., van Bavel, B., Lindström, G., Becher, G. 2010a. Levels in food and beverages and daily intake of fluorinated compounds in Norway. Chemosphere, 80, 1137-1143.

Haug, L.S., Thomsen, C., Brantsæter, A.L., Kvalem, H.E., Haugen, M., Becker, G., Alexander, J., Meltzer, H.M., Knutsen, H.K. 2010b. Diet and particulary seafood are major sources of perfluorinated compounds in humans. Environ. Int., 36, 772-778.

Hölzer, J., Midasch, O., Rauchfuss, K., Kraft, M., Reupert, R., Angerer, J., Kleeschulte, P., Marschall, N. and Wilhelm, M. 2008. Biomonitering of perfluorinated compounds in children and adults exposed to perfluooctanoate-contaminated drinking water, Environmental Health Perspectives, vol 116 (5), 651-657.

Mons, M., van Roon, A., de Voogt, P. 2007. Perfluoroalkylated substances in Dutch drinking water sources, KIWA water, BTO 07.048, Nieuwegein, 2007.

Rylander, C., Brustad, M., Falk, H., Sandanger, T. M. 2009. Dietary predictors and plasma concentartions of pefluorinated compounds in a costal population from Nothern Norway. Jour of Environmental and Public Health, vol 2009, Article ID 268219, 10 pages.

Schuetze, A., Heberer, T., Effkemann, S., Juergensen, S., 2010. Occurrence and assessment of perfluorinated chemicals in wild fish from Nothern Germany. Chemosphere, 78, 647-652.

Skutlarek, D., Exner, M., Farber, H. 2006. Perfluoinated surfactants in surface and drinking water. Environ Sci Pollut Res Int, vol 13 (5), 299-307.

Tittlemier, S.A., Pepper, K., Edwards, L. 2006. Concentrations of perfluorooctanesulfonamides in Canadian Total Diet Study composite food samples collected between 1992-2004. J. Agric Food Chem, 54, 8385-8389.

Trier, X. 2011. Polyfluorinated surfactants in food packaging of paper and board. Ph.D. thesis.

Trier, X. et al., 2011. Polyfluorinated surfactants (PFS) in paper and board coatings for food pakaging. Environ Sci Poll Res, 18, 1108-1120.

Trier, X., Pedersen, G. A., Granby, K. 2012. A Danish survey: Screening of fluorinated substances in food contact materials of paper and board (results not yet publiched).

Trudel, D., Horowitz, L., Wormuth, M., Scheringer, M., Cousins, I. T. and Hunderbühler, K. 2008. Estmating Consumer Exposure to PFOS and PFOA. Risk Analysis, Vol 28, 2, 251-269. 
Ullah, S., Alsberg, T., Berger, U., 2011. Simultaneous determination of perfluoroalkyl phosphonates, caboxylates and sulfonates in drinking water. Jour of Chromatography A, 1218, 6388-6395.

Vestergren, R., Cousins, I.T., Trudel, D., Wormuth, M. and Scheringer, M. 2008. Estimating the contribution of precursor compounds in consumer exposure to PFOS and PFOA. Chemosphere 73, 1617-1624.

Vestergren, R., Cousins, I.T. 2009. Tracking the Pathways of Human Exposure to Perfluorocarboxylates. Environmental Science \& Technology 43 (15), 5565-5575.

\section{Consumer products}

3M. 2010. Scotchgard Carpet \& Rug protector (1023-17N). Material safety data sheet.

Barber, J.L., Berger, U., Chaemfa, C., Huber, S., Jahnke, A., Temme, C., Jones, K. 2007. Analysis of per-and polyfluorinated alkyl substances in air samples from Northwest Europe. J. Environ. Monitor, 9, 530-541.

Berger, U., Herzke, D. 2006. Per- and polyfluorinated alkyl substances (PFAS) extracted from textile samples. Organohalogen compounds, 68, 2023-2026.

Castro, I.C., Briceno, J.C. 2010. Perfluorocarbon-based oxygen carriers: Review of Products and trials. Artif. organs, 34, 622-634.

Cinglasan-Panlilio, M.J.A., Mabury, S.A. 2006. Significant residual fluorinated alcohols present in various fluorinated materials. Environ. Sci. Technol., 40, 1447-1453.

DuPont. 2004. DuPont Teflon Advanced carpet and upholstery protection. Usage information.

DuPont. 2005. Teflon Advanced. Safety Data Sheet.

Fraser, A.J., Webster, T.F., Watkins, D.F., Nelson, J.W., Stapelton, H.J., Calafat, A.M., Kato, K., Shoeib, M., Vieria, V.M., McClean, M.D. 2012. Polyfluorinated compounds in serum linked to indoor air in office environments. Environ. Sci. Technol., 46, 1209-1215.

Gelest. 2005. SiBRID fluorocarbon silicone. Product information.

Goosey, E., Harrad, S. 2011. Perfluoroalkyl compounds in dust from Asian, Australian, European, and North American homes and UK cars, classrooms, and offices. Environ. Int., 37, 86-92.

Goosey, E., Harrad, S. 2012. Perfluoroalkyl substances in UK indoor and outdoor air: Spatial and seasonal variation, and implications for human exposure. Environ. Int., 45, 86-90.

Haug, L.S., Huber, S., Schlabach, M., Becher, G., Thomsen, C. 2011. Investigation on per-and polyfluorinated compounds in paired samples of house dust and indoor air from Norwegian homes. Environ. Sci. Technol., 45, 7991-8.

Herzke, D., Olsson, E., Posner, S. 2012. Perfluoroalkyl and polyfluoroalkyl substances (PFASs) in consumer products in Norway - A pilot study. Chemosphere, 88, 980-987.

Huber, S., Haug, L.S., Schlabach, M. 2011. Per-and polyfluorinated compounds in house dust and indoor air from northern Norway - a pilot study. Chemosphere, 84, 1686-93.

Jahnke, A., Ahrens, L., Ebinghaus, R., Temme, C. 2007. Urban versus remote air concentrations of fluorotelomer alcohols and other perfluorinated alkyl substances in Germany. Environ. Sci. Technol., 41, 745-752

Jensen, A.A., Poulsen, P.B., Bossi, R. 2008. Survey and environmental/health assessment of fluorinated substances in impregnated consumer products and impregnating agents. Survey of Chemical Substances in Consumer Products, No. 99. Danish Environmental Protection Agency. 
Jogsten, I.E., Nadal, M., van Bavel, B., Lindström, G., Domingo, J.L. 2012. Per- and polyfluorinated compounds (PFCs) in house dust and indor air in Catalonia Spain: Implications for human exposure. Environ. Int., 39, 172-180.

Kato, K., Calafat, A.M., Needham, L.L. 2009. Polyfluoroalkyl chemicals in house dust. Environ. Res., 109, 518-23.

Key, B.D., Howell, R.D., Criddle, C.S. 1997. Fluorinated organics in the biosphere. Environ. Sci. Technol., 31, 2445-2454.

Kim, S.K., Shoeib, M., Kim, K.S., Park, J.E. 2012. Indoor and outdoor poly- and perfluoroalkyl substances (PFASs) in Korea determined by passive air sampler. Environ. Pollut., 162, 144-150.

Langer, V., Dreyer, A., Ebinghaus, R. 2010. Polyfluorinated compounds in residential and nonresidential indoor air. Environ. Sci. Technol., 44, 8075-81.

Liu, W., Takahashi, S., Sakuramachi, Y., Harada, K.H., Koizumi, A. 2012. Polyfluorinated telomers in indoor air of Japanese houses. Chemosphere, In press.

Moe, M.K., Huber, S., Svenson, J., Hagenaars, A., Pabon, M., Trümper, M., Berger, U., Knapen, D., Herzke, D. 2012. The structure of the fire fighting foam surfactant Forafac1157 and its biological and photolytic transformation products. Chemosphere, 89, 869-875.

Shoeib, M., Harner, T., Ikonomou, M., Kannan, K. 2004. Indoor and outdoor air concentrations and phase partitioning of perfluoroalkylsulfonamides and polybrominated diphenyl ethers. Environ. Sci. Technol., 38, 131-1320.

Shoeib, M., Harner, T., Webster, G.M., Lee, S.C. 2011. Indoor sources of poly- and perfluorinated compounds (PFCs) in Vancouver, Canada: implications for human exposure. Environ. Sci. Technol., 45, 7999-8005.

Shoeib, M., Harner, T., Zhu, J. 2007. Indoor air \& dust concentrations of fluorotelomer alcohols. Organohalogen Compounds, 69, 146-149.

Shoeib, M.,Harner, T., Wilford, B.H., Jones, K.C., Zhu, J. 2005. Perfluorinated sulfonamides in indoor and outdoor air and indoor dust: Occurence, partitioning, and human exposure. Environ. Sci. Technol., 39, 6599-6606.

Sinclair, E., Kim, S.K., Akinleye, H.B., Kannan, K. 2007. Quantitation of gas-phase perfluoroalkyl surfactants and fluorotelomer alcohols released from nonstick cookware and microwave popcorn bags. Environ. Sci. Technol., 41, 1180-1185.

Strynar, M.J., Lindstrom, A.B. 2008. Perfluorinated compounds in house dust from Ohio and North Carolina, USA. Environ. Sci. Technol., 42, 3751-6.

Vorob'ev, S.I. 2009. First- and second-generation perfluorocarbon emulsions. Pharm. Chem. J.-USSR, 43, 30-40.

Yang, Z., Price, C., Bosco, G., Tucci, M., El-Badri, N.S., Mangar, D., Camporesi, E.M. 2008. The effect of isovolemic hemodilution with Oxycyte, a perfluorocarbo emulsion, on cerebral blood flow in rats. PLOS one, 3, 1-5.

Zaggia, A., Ameduri, B. 2012. Recent advances on synthesis of potentially nonbioaccumulable fluorinated sufractants. Current Opinion in Colloid \& Interface Science, 17, 188-195. 


\section{Occurrence of PFCs in human}

Bonefeld-Jorgensen, E.C., Long, M., Bossi, R., Ayotte, P., Asmund, G., Kruger, T., Ghisari, M., Mulvad, G., Kern, P., Nzulumiki, P., Dewailly, E. 2011. Perfluorinated compounds are related to breast cancer risk in Greenlandic Inuit: a case control study. Environ Health 10, 88.

D’Eon, J.C., Crozier, P.W., Furdui, V.I., Reiner, E.J., Libelo, E.L., Mabury, S.A. 2009. Observation of a commercial fluorinated material, the polyfluoroalkyl phosphoric acid diesters, in human sera, wastewater treatment plant sludge, and paper fibers. Environmental science \& technology 43, 4589-4594.

Dinglasan, M.J., Ye, Y., Edwards, E.A., Mabury, S.A. 2004. Fluorotelomer alcohol biodegradation yields poly- and perfluorinated acids. Environmental science \& technology 38, 2857-2864.

Ehresman, D.J., Froehlich, J.W., Olsen, G.W., Chang, S.C., Butenhoff, J.L. 2007. Comparison of human whole blood, plasma, and serum matrices for the determination of perfluorooctanesulfonate (PFOS), perfluorooctanoate (PFOA), and other fluorochemicals. Environmental research 103, 176-184.

Fei, C., McLaughlin, J.K., Tarone, R.E., Olsen, J. 2007. Perfluorinated chemicals and fetal growth: a study within the Danish National Birth Cohort. Environ Health Perspect 115, 1677-1682.

Fraser, A.J., Webster, T.F., Watkins, D.J., Nelson, J.W., Stapleton, H.M., Calafat, A.M., Kato, K., Shoeib, M., Vieira, V.M., McClean, M.D. 2012. Polyfluorinated compounds in serum linked to indoor air in office environments. Environmental science \& technology 46, 1209-1215.

Freberg, B.I., Haug, L.S., Olsen, R., Daae, H.L., Hersson, M., Thomsen, C., Thorud, S., Becher, G., Molander, P., Ellingsen, D.G. 2010 Occupational exposure to airborne perfluorinated compounds during professional ski waxing. Environ Sci Technol 44, 7723-7728.

Fromme, H., Tittlemier, S.A., Völkel, W., Wilhelm, M., Twardella, D. 2009. Perfluorinated compounds - Exposure assessment for the general population in western countries. Int J Hyg Environ Health 212, 239-270.

Glynn, A., Berger, U., Bignert, A., Ullah, S., Aune, M., Lignell, S., Darnerud, P.O. 2012. Perfluorinated Alkyl Acids in Blood Serum from Primiparous Women in Sweden: Serial Sampling during Pregnancy and Nursing, And Temporal Trends 1996-2010. Environ Sci Technol 46, 9071-9079.

Gutzkow, K.B., Haug, L.S., Thomsen, C., Sabaredzovic, A., Becher, G., Brunborg, G. 2012. Placental transfer of perfluorinated compounds is selective - a Norwegian Mother and Child sub-cohort study. Int J Hyg Environ Health 215, 216-219.

Halldorsson, T.I., Fei, C., Olsen, J., Lipworth, L., McLaughlin, J.K., Olsen, S.F. 2008. Dietary predictors of perfluorinated chemicals: a study from the Danish National Birth Cohort. Environ Sci Technol 42, 8971-8977.

Haug, L.S., Huber, S., Becher, G., Thomsen, C. 2011. Characterisation of human exposure pathways to perfluorinated compounds - comparing exposure estimates with biomarkers of exposure. Environ Int 37, 687-693.

Haug, L.S., Thomsen, C., Becher, G. 2009. Time trends and the influence of age and gender on serum concentrations of perfluorinated compounds in archived human samples. Environ Sci Technol 43, 2131-2136.

Joensen, U.N., Bossi, R., Leffers, H., Jensen, A.A., Skakkebaek, N.E., Jorgensen, N. 2009. Do perfluoroalkyl compounds impair human semen quality? Environ Health Perspect 117, 923-927. 
Jones, P.D., Hu, W., De Coen, W., Newsted, J.L., Giesy, J.P. 2003 Binding of perfluorinated fatty acids to serum proteins. Environ Toxicol Chem 22, 2639-2649.

Karrman, A., Ericson, I., van Bavel, B., Darnerud, P.O., Aune, M., Glynn, A., Lignell, S., Lindstrom, G. 2007a. Exposure of perfluorinated chemicals through lactation: levels of matched human milk and serum and a temporal trend, 1996-2004, in Sweden. Environ Health Perspect 115, 226-230.

Karrman, A., Langlois, I., van Bavel, B., Lindstrom, G., Oehme, M. 2007b. Identification and pattern of perfluorooctane sulfonate (PFOS) isomers in human serum and plasma. Environ Int 33, 782-788.

Karrman, A., van Bavel, B., Jarnberg, U., Hardell, L., Lindstrom, G. 2006. Perfluorinated chemicals in relation to other persistent organic pollutants in human blood. Chemosphere 64, 1582-1591.

Kato, K., Wong, L.Y., Jia, L.T., Kuklenyik, Z., Calafat, A.M. 2011. Trends in exposure to polyfluoroalkyl chemicals in the U.S. Population: 1999-2008. Environ Sci Technol 45, 8037-8045.

Lau, C. 2012. Perfluoroalkyl acids: Recent research highlights. Reprod Toxicol 33, 405-409.

Lee, H., Mabury, S.A. 2011. A pilot survey of legacy and current commercial fluorinated chemicals in human sera from United States donors in 2009. Environmental science \& technology 45, 8067-8074.

Lindh, C.H., Rylander, L., Toft, G., Axmon, A., Rignell-Hydbom, A., Giwercman, A., Pedersen, H.S., Goalczyk, K., Ludwicki, J.K., Zvyezday, V., Vermeulen, R., Lenters, V., Heederik, D., Bonde, J.P., Jonsson, B.A. 2012. Blood serum concentrations of perfluorinated compounds in men from Greenlandic Inuit and European populations. Chemosphere 88, 1269-1275.

Long, M., Bossi, R., Bonefeld-Jorgensen, E.C. 2012. Level and temporal trend of perfluoroalkyl acids in Greenlandic Inuit. Int J Circumpolar Health 71, 17998.

Needham, L.L., Grandjean, P., Heinzow, B., Jørgensen, P.J., Nielsen, F., Patterson, D.G., Sjödin, A., Turner, W.E., Weihe, P. 2010. Partition of Environmental Chemicals between Maternal and Fetal Blood and Tissues. Environ Sci Technol 45, 1121-1126.

Nilsson, H., Karrman, A., Rotander, A., van Bavel, B., Lindstrom, G., Westberg, H. 2010a. Inhalation exposure to fluorotelomer alcohols yield perfluorocarboxylates in human blood? Environ Sci Technol 44, 7717-7722.

Nilsson, H., Karrman, A., Westberg, H., Rotander, A., van Bavel, B., Lindstrom, G. $2010 \mathrm{~b}$. A time trend study of significantly elevated perfluorocarboxylate levels in humans after using fluorinated ski wax. Environ Sci Technol 44, 2150-2155.

Nilsson, S., Makela, S., Treuter, E., Tujague, M., Thomsen, J., Andersson, G., Enmark, E., Pettersson, K., Warner, M., Gustafsson, J.A. 2001. Mechanisms of estrogen action. Physiol Rev 81, 1535-1565.

Rylander, C., Brustad, M., Falk, H., Sandanger, T.M. 2009. Dietary predictors and plasma concentrations of perfluorinated compounds in a coastal population from northern Norway. J Environ Public Health 2009, 268219.

Rotander, A., Kärrman, A., van Bavel, B., Polder, A., Rigét, F., Auðunsson, G.A., Víkingsson, G., Gabrielsen, G.W., Bloch, D. \& Dam, M.; Increasing levels of long-chain perfluorocarboxylic acids (PFCAs) in Arctic and North Atlantic marine mammals, 19842009. Chemosphere, 86(3): 278-285. 
Tiido, T., Rignell-Hydbom, A., Jonsson, B.A., Giwercman, Y.L., Pedersen, H.S., Wojtyniak, B., Ludwicki, J.K., Lesovoy, V., Zvyezday, V., Spano, M., Manicardi, G.C., Bizzaro, D., Bonefeld-Jorgensen, E.C., Toft, G., Bonde, J.P., Rylander, L., Hagmar, L., Giwercman, A. 2006. Impact of PCB and p, $p^{\prime}-D D E$ contaminants on human sperm $Y: X$ chromosome ratio: studies in three European populations and the Inuit population in Greenland. Environ Health Perspect 114, 718-724.

Thomsen, C., Haug, L.S., Stigum, H., Frøshaug, M., Broadwell, S.L., Becher, G., 2010. Changes in concentrations of perfluorinated compounds, polybrominated diphenyl ethers and polychlorinated biphenyls in Norwegian breast-milk during twelve months of lactation. Environ. Sci. Technol. 44, 9550-9556.

Vestergaard, S., Nielsen, F., Andersson, A.M., Hjollund, N.H., Grandjean, P., Andersen, H.R., Jensen, T.K. 2012. Association between perfluorinated compounds and time to pregnancy in a prospective cohort of Danish couples attempting to conceive. Hum Reprod 27, 873-880.

Weihe, P., Kato, K., Calafat, A.M., Nielsen, F., Wanigatunga, A.A., Needham, L.L., Grandjean, P. 2008. Serum concentrations of polyfluoroalkyl compounds in Faroese whale meat consumers. Environ Sci Technol 42, 6291-6295.

\section{Human toxicity and environmental effects}

(ECHA), E.C.A., 2008. Summary Of Classification and Labelling: Harmonised classification - Annex VI of Regulation (EC) No 1272/2008 (CLP Regulation) - PFOS. vol. 2012.

(ECHA), E.C.A., 2011. Opinions of the Committee for Risk Assessment on proposals for harmonised classification and labelling - PFOA. vol. 2012.

(ECHA), E.C.A., 2012a. Registry of current Harmonised Classification and Labelling intentions: heptadecafluorodecanoic acid. vol. 2012.

(ECHA), E.C.A., 2012b. Registry of current Harmonised Classification and Labelling intentions: nonadecafluorononanoic acid. vol. 2012.

Abbott, B.D., Wolf, C.J., Schmid, J.E., Das, K.P., Zehr, R.D., Helfant, L., Nakayama, S., Lindstrom, A.B., Strynar, M.J., Lau, C., 2007. Perfluorooctanoic acid induced developmental toxicity in the mouse is dependent on expression of peroxisome proliferator activated receptor-alpha. Toxicol Sci 98, 571-581.

Apelberg, B.J., Witter, F.R., Herbstman, J.B., Calafat, A.M., Halden, R.U., Needham, L.L., Goldman, L.R., 2007. Cord serum concentrations of perfluorooctane sulfonate (PFOS) and perfluorooctanoate (PFOA) in relation to weight and size at birth. Environ Health Perspect 115, 1670-1676.

Begley, T.H., White, K., Honigfort, P., Twaroski, M.L., Neches, R., Walker, R.A., 2005. Perfluorochemicals: potential sources of and migration from food packaging. Food additives and contaminants 22, 1023-1031.

Bennett, D.H., McKone, T.E., Matthies, M., Kastenberg, W.E., 1998. General formulation of characteristic travel distance for semivolatile organic chemicals in a multimedia environment. Environ Sci Technol 32, 4023-4030.

Benninghoff, A.D., Bisson, W.H., Koch, D.C., Ehresman, D.J., Kolluri, S.K., Williams, D.E., 2011. Estrogen-like activity of perfluoroalkyl acids in vivo and interaction with human and rainbow trout estrogen receptors in vitro. Toxicol Sci 120, 42-58.

Benninghoff, A.D., Orner, G.A., Buchner, C.H., Hendricks, J.D., Duffy, A.M., Williams, D.E., 2012. Promotion of Hepatocarcinogenesis by Perfluoroalkyl Acids in Rainbow Trout. Toxicol Sci 125, 69-78.

Beyer, A., Mackay, D., Matthies, M., Wania, F., Webster, E., 2000. Assessing long-range transport potential of persistent organic pollutants. Environ Sci Technol 34, 699-703. 
Biegel, L.B., Hurtt, M.E., Frame, S.R., O'Connor, J.C., Cook, J.C., 2001. Mechanisms of extrahepatic tumor induction by peroxisome proliferators in male CD rats. Toxicol Sci 60, 44-55.

Biegel, L.B., Liu, R.C.M., Hurtt, M.E., Cook, J.C., 1995. Effects of Ammonium Perfluorooctanoate on Leydig-Cell Function: In Vitro, in Vivo, and ex Vivo Studies. Toxicol Appl Pharmacol 134, 18-25.

Bloom, M.S., Kannan, K., Spliethoff, H.M., Tao, L., Aldous, K.M., Vena, J.E., 2010. Exploratory assessment of perfluorinated compounds and human thyroid function. Physiology \& behavior 99, 240-245.

Bonefeld-Jorgensen, E.C., Long, M., Bossi, R., Ayotte, P., Asmund, G., Kruger, T., Ghisari, M., Mulvad, G., Kern, P., Nzulumiki, P., Dewailly, E., 2011. Perfluorinated compounds are related to breast cancer risk in Greenlandic Inuit: a case control study. Environ Health 10, 88.

Bookstaff, R.C., Moore, R.W., Ingall, G.B., Peterson, R.E., 1990. Androgenic deficiency in male rats treated with perfluorodecanoic acid. Toxicol Appl Pharmacol 104, 322-333.

Brandsma, S.H., Smithwick, M., Solomon, K., Small, J., de Boer, J., Muir, D.C.G., 2011. Dietary exposure of rainbow trout to 8:2 and 10:2 fluorotelomer alcohols and perfluorooctanesulfonamide: Uptake, transformation and elimination. Chemosphere 82, 253-258.

Breivik, K., Sweetman, A., Pacyna, J.M., Jones, K.C., 2007. Towards a global historical emission inventory for selected PCB congeners - A mass balance approach. 3 . An update. Science of the Total Environment 377, 296-307.

Breivik, K., Wania, F., Muir, D.C.G., Alaee, M., Backus, S., Pacepavicius, G., 2006. Empirical and modeling evidence of the long-range atmospheric transport of decabromodiphenyl ether. Environ Sci Technol 40, 4612-4618.

Brewster, D.W., Birnbaum, L.S., 1989. The biochemical toxicity of perfluorodecanoic acid in the mouse is different from that of 2,3,7,8-tetrachlorodibenzo-p-dioxin. Toxicol Appl Pharmacol 99, 544-554.

Butenhoff, J., Costa, G., Elcombe, C., Farrar, D., Hansen, K., Iwai, H., Jung, R., Kennedy, G., Jr., Lieder, P., Olsen, G., Thomford, P., 2002. Toxicity of ammonium perfluorooctanoate in male cynomolgus monkeys after oral dosing for 6 months. Toxicol Sci 69, 244-257.

Butenhoff, J.L., Bjork, J.A., Chang, S.C., Ehresman, D.J., Parker, G.A., Das, K., Lau, C., Lieder, P.H., van Otterdijk, F.M., Wallace, K.B., 2012. Toxicological evaluation of ammonium perfluorobutyrate in rats: twenty-eight-day and ninety-day oral gavage studies. Reprod Toxicol 33, 513-530.

Butenhoff, J.L., Chang, S.C., Ehresman, D.J., York, R.G., 2009. Evaluation of potential reproductive and developmental toxicity of potassium perfluorohexanesulfonate in Sprague Dawley rats. Reprod Toxicol 27, 331-341.

Butenhoff, J.L., Kennedy, G.L., Jr., Hinderliter, P.M., Lieder, P.H., Jung, R., Hansen, K.J., Gorman, G.S., Noker, P.E., Thomford, P.J., 2004. Pharmacokinetics of perfluorooctanoate in cynomolgus monkeys. Toxicol Sci 82, 394-406.

Cassone, C. G., Vongphachan, V., Chiu, S., Williams, K.L., Letcher, R.J., Pelletier, E., Crump, D., Kennedy, S.W., 2012. In ovo effects of perfluorohexane sulfonate and perfluorohexanoate on pipping success, development, mrna expression, and thyroid hormone levels in chicken embryos. Toxicol Sci 127(1): 216-224.

Chang, S.C., Das, K., Ehresman, D.J., Ellefson, M.E., Gorman, G.S., Hart, J.A., Noker, P.E., Tan, Y.M., Lieder, P.H., Lau, C., Olsen, G.W., Butenhoff, J.L., 2008. Comparative pharmacokinetics of perfluorobutyrate in rats, mice, monkeys, and humans and relevance to human exposure via drinking water. Toxicol Sci 104, 40-53. 
Chen, M.H., Ha, E.H., Wen, T.W., Su, Y.N., Lien, G.W., Chen, C.Y., Chen, P.C., Hsieh, W.S., 2012. Perfluorinated compounds in umbilical cord blood and adverse birth outcomes. PLoS One 7, e42474.

Chengelis, C.P., Kirkpatrick, J.B., Radovsky, A., Shinohara, M., 2009. A 90-day repeated dose oral (gavage) toxicity study of perfluorohexanoic acid ( $\mathrm{PFHxA}$ ) in rats (with functional observational battery and motor activity determinations). Reprod Toxicol 27, 342-351.

Christensen, K.Y., Maisonet, M., Rubin, C., Holmes, A., Calafat, A.M., Kato, K., Flanders, W.D., Heron, J., McGeehin, M.A., Marcus, M., 2011. Exposure to polyfluoroalkyl chemicals during pregnancy is not associated with offspring age at menarche in a contemporary British cohort. Environ Int 37, 129-135.

Courrier, H.M., Krafft, M.P., Butz, N., Porté, C., Frossard, N., Rémy-Kristensen, A., Mély, Y., Pons, F., Vandamme, T.F., 2003. Evaluation of cytotoxicity of new semi-fluorinated amphiphiles derived from dimorpholinophosphate. Biomaterials 24, 689-696.

D'Eon J, C., Mabury, S.A., 2011. Exploring indirect sources of human exposure to perfluoroalkyl carboxylates (PFCAs): evaluating uptake, elimination, and biotransformation of polyfluoroalkyl phosphate esters (PAPS) in the rat. Environ Health Perspect 119, 344-350.

D’Eon, J.C., Crozier, P.W., Furdui, V.I., Reiner, E.J., Libelo, E.L., Mabury, S.A., 2009. Observation of a commercial fluorinated material, the polyfluoroalkyl phosphoric acid diesters, in human sera, wastewater treatment plant sludge, and paper fibers. Environmental science \& technology 43, 4589-4594.

D’Eon, J.C., Mabury, S.A., 2007. Production of perfluorinated carboxylic acids (PFCAs) from the biotransformation of polyfluoroalkyl phosphate surfactants (PAPS): exploring routes of human contamination. Environ Sci Technol 41, 4799-4805.

Dallaire, R., Dewailly, E., Pereg, D., Dery, S., Ayotte, P., 2009. Thyroid function and plasma concentrations of polyhalogenated compounds in Inuit adults. Environ Health Perspect 117, 1380-1386.

Das, K.P., Grey, B.E., Zehr, R.D., Wood, C.R., Butenhoff, J.L., Chang, S.C., Ehresman, D.J., Tan, Y.M., Lau, C., 2008. Effects of perfluorobutyrate exposure during pregnancy in the mouse. Toxicol Sci 105, 173-181.

Dinglasan-Panlilio, M.J.A., Mabury, S.A., 2006. Significant Residual Fluorinated Alcohols Present in Various Fluorinated Materials. Environmental Science \& Technology 40, 1447-1453.

Dinglasan, M.J., Ye, Y., Edwards, E.A., Mabury, S.A., 2004. Fluorotelomer alcohol biodegradation yields poly- and perfluorinated acids. Environmental science \& technology 38, 2857-2864.

DuPont, 2012. Teflon - Uses and Applications.

Ehresman, D.J., Froehlich, J.W., Olsen, G.W., Chang, S.C., Butenhoff, J.L., 2007. Comparison of human whole blood, plasma, and serum matrices for the determination of perfluorooctanesulfonate (PFOS), perfluorooctanoate (PFOA), and other fluorochemicals. Environmental research 103, 176-184.

Emmett, E.A., Zhang, H., Shofer, F.S., Freeman, D., Rodway, N.V., Desai, C., Shaw, L.M., 2006. Community exposure to perfluorooctanoate: relationships between serum levels and certain health parameters. Journal of occupational and environmental medicine / American College of Occupational and Environmental Medicine 48, 771-779.

Eriksen, K.T., Raaschou-Nielsen, O., Sørensen, M., Roursgaard, M., Loft, S., Møller, P., 2010. Genotoxic potential of the perfluorinated chemicals PFOA, PFOS, PFBS, PFNA and PFHXA in human HepG2 cells. Mutation Research. Genetic Toxicology and Environmental Mutagenesis 700, 39-43. 
Eriksen, K.T., Sorensen, M., McLaughlin, J.K., Lipworth, L., Tjonneland, A., Overvad, K., Raaschou-Nielsen, O., 2009. Perfluorooctanoate and perfluorooctanesulfonate plasma levels and risk of cancer in the general Danish population. J Natl Cancer Inst 101, 605-609.

Fang, X., Feng, Y., Wang, J., Dai, J., 2010. Perfluorononanoic acid-induced apoptosis in rat spleen involves oxidative stress and the activation of caspase-independent death pathway. Toxicology 267, 54-59.

Fang, X., Gao, G., Xue, H., Zhang, X., Wang, H., 2012. Exposure of perfluorononanoic acid suppresses the hepatic insulin signal pathway and increases serum glucose in rats. Toxicology 294, 109-115.

Fang, X., Zhang, L., Feng, Y., Zhao, Y., Dai, J., 2008. Immunotoxic effects of perfluorononanoic acid on BALB/c mice. Toxicol Sci 105, 312-321.

Fasano, W.J., Sweeney, L.M., Mawn, M.P., Nabb, D.L., Szostek, B., Buck, R.C., Gargas, M.L., 2009. Kinetics of 8-2 fluorotelomer alcohol and its metabolites, and liver glutathione status following daily oral dosing for 45 days in male and female rats. Chem Biol Interact 180, 281-295.

Fei, C., McLaughlin, J.K., Lipworth, L., Olsen, J., 2009. Maternal levels of perfluorinated chemicals and subfecundity. Hum Reprod 24, 1200-1205.

Fei, C., McLaughlin, J.K., Lipworth, L., Olsen, J., 2010. Prenatal exposure to PFOA and PFOS and risk of hospitalization for infectious diseases in early childhood. Environ Res 110, 773-777.

Fei, C., McLaughlin, J.K., Tarone, R.E., Olsen, J., 2007. Perfluorinated chemicals and fetal growth: a study within the Danish National Birth Cohort. Environ Health Perspect 115, 1677-1682.

Fei, C., McLaughlin, J.K., Tarone, R.E., Olsen, J., 2008. Fetal growth indicators and perfluorinated chemicals: a study in the Danish National Birth Cohort. Am J Epidemiol 168, 66-72.

Fei, C., Olsen, J., 2011. Prenatal exposure to perfluorinated chemicals and behavioral or coordination problems at age 7 years. Environ Health Perspect 119, 573-578.

Fernandez Freire, P., Perez Martin, J.M., Herrero, O., Peropadre, A., de la Pena, E., Hazen, M.J., 2008. In vitro assessment of the cytotoxic and mutagenic potential of perfluorooctanoic acid. Toxicol In Vitro 22, 1228-1233.

Fiedler, S., Pfister, G., Schramm, K.-W., 2010. Poly- and perfluorinated compounds in household consumer products. Toxicological \& Environmental Chemistry 92, 1801-1811.

Fraser, A.J., Webster, T.F., Watkins, D.J., Nelson, J.W., Stapleton, H.M., Calafat, A.M., Kato, K., Shoeib, M., Vieira, V.M., McClean, M.D., 2012. Polyfluorinated compounds in serum linked to indoor air in office environments. Environmental science \& technology 46, 1209-1215.

Freberg, B.I., Haug, L.S., Olsen, R., Daae, H.L., Hersson, M., Thomsen, C., Thorud, S., Becher, G., Molander, P., Ellingsen, D.G., 2010. Occupational exposure to airborne perfluorinated compounds during professional ski waxing. Environ Sci Technol 44, 7723-7728.

Frisbee, S.J., Shankar, A., Knox, S.S., Steenland, K., Savitz, D.A., Fletcher, T., Ducatman, A.M., 2010. Perfluorooctanoic acid, perfluorooctanesulfonate, and serum lipids in children and adolescents: results from the C8 Health Project. Arch Pediatr Adolesc Med 164, 860-869.

Fromme, H., Tittlemier, S.A., Völkel, W., Wilhelm, M., Twardella, D., 2009. Perfluorinated compounds - Exposure assessment for the general population in western countries. Int J Hyg Environ Health 212, 239-270. 
Galatius, A., Dietz, R., Rigét, F.F., Sonne, C., Kinze, C.C., Lockyer, C., Bossi, R., 2011. Temporal and life history related trends of perfluorochemicals in harbor porpoises from the danish north sea. Mar. Poll. Bull. 62(7): 1476-1483.

Gallo, V., Leonardi, G., Genser, B., Lopez-Espinosa, M.J., Frisbee, S.J., Karlsson, L., Ducatman, A.M., Fletcher, T., 2012. Serum perfluorooctanoate (PFOA) and perfluorooctane sulfonate (PFOS) concentrations and liver function biomarkers in a population with elevated PFOA exposure. Environ Health Perspect 120, 655-660.

Gewurtz, S.B., Bhavsar, S.P., Crozier, P.W., Diamond, M.L., Helm, P.A., Marvin, C.H., Reiner, E.J., 2009. Perfluoroalkyl contaminants in window film: indoor/outdoor, urban/rural, and winter/summer contamination and assessment of carpet as a possible source. Environ Sci Technol 43, 7317-7323.

Gilliland, F.D., Mandel, J.S., 1993. Mortality among employees of a perfluorooctanoic acid production plant. Journal of occupational medicine : official publication of the Industrial Medical Association 35, 950-954.

Glynn, A., Berger, U., Bignert, A., Ullah, S., Aune, M., Lignell, S., Darnerud, P.O., 2012. Perfluorinated Alkyl Acids in Blood Serum from Primiparous Women in Sweden: Serial Sampling during Pregnancy and Nursing, And Temporal Trends 1996-2010. Environ Sci Technol 46, 9071-9079.

Goorha, Y.K., Deb, P., Chatterjee, T., Dhot, P.S., Prasad, R.S., 2003. Artifical blood. Medical Journal Armed Forces India 59, 45-50.

Grandjean, P., Andersen, E.W., Budtz-Jorgensen, E., Nielsen, F., Molbak, K., Weihe, P., Heilmann, C., 2012. Serum vaccine antibody concentrations in children exposed to perfluorinated compounds. JAMA 307, 391-397.

Grice, M.M., Alexander, B.H., Hoffbeck, R., Kampa, D.M., 2007. Self-reported medical conditions in perfluorooctanesulfonyl fluoride manufacturing workers. Journal of occupational and environmental medicine / American College of Occupational and Environmental Medicine 49, 722-729.

Gump, B.B., Wu, Q., Dumas, A.K., Kannan, K., 2011. Perfluorochemical (PFC) exposure in children: associations with impaired response inhibition. Environ Sci Technol 45, 8151-8159.

Gutzkow, K.B., Haug, L.S., Thomsen, C., Sabaredzovic, A., Becher, G., Brunborg, G., 2012. Placental transfer of perfluorinated compounds is selective - a Norwegian Mother and Child sub-cohort study. Int J Hyg Environ Health 215, 216-219.

Hagenaars, A., Meyer, I.J., Herzke, D., Pardo, B.G., Martinez, P., Pabon, M., De Coen, W., Knapen, D., 2011a. The search for alternative aqueous film forming foams (AFFF) with a low environmental impact: Physiological and transcriptomic effects of two Forafac $\AA$ fluorosurfactants in turbot. Aquatic Toxicology 104, 168-176.

Hagenaars, A., Vergauwen, L., De Coena, W., Knapen, D., 2011b. Structure-activity relationship assessment of four perfluorinated chemicals using a prolonged zebrafish early life stage test. Chemosphere 82(5): 764-772.

Halldorsson, T.I., Fei, C., Olsen, J., Lipworth, L., McLaughlin, J.K., Olsen, S.F., 2008. Dietary predictors of perfluorinated chemicals: a study from the Danish National Birth Cohort. Environ Sci Technol 42, 8971-8977.

Halldorsson, T.I., Rytter, D., Haug, L.S., Bech, B.H., Danielsen, I., Becher, G., Henriksen, T.B., Olsen, S.F., 2012. Prenatal exposure to perfluorooctanoate and risk of overweight at 20 years of age: a prospective cohort study. Environ Health Perspect 120, 668-673.

Hamm, M.P., Cherry, N.M., Chan, E., Martin, J.W., Burstyn, I., 2010. Maternal exposure to perfluorinated acids and fetal growth. J Expo Sci Environ Epidemiol 20, 589-597. 
Harris, M.W., Birnbaum, L.S., 1989. Developmental toxicity of perfluorodecanoic acid in $C 57 B L / 6 N$ mice. Fundamental and applied toxicology : official journal of the Society of Toxicology 12, 442-448.

Harris, M.W., Uraih, L.C., Birnbaum, L.S., 1989. Acute toxicity of perfluorodecanoic acid in C57BL/6 mice differs from 2,3,7,8-tetrachlorodibenzo-p-dioxin. Fundamental and applied toxicology : official journal of the Society of Toxicology 13, 723-736.

Haug, L.S., Huber, S., Becher, G., Thomsen, C., 2011. Characterisation of human exposure pathways to perfluorinated compounds - comparing exposure estimates with biomarkers of exposure. Environ Int 37, 687-693.

Haug, L.S., Thomsen, C., Becher, G., 2009. Time trends and the influence of age and gender on serum concentrations of perfluorinated compounds in archived human samples. Environ Sci Technol 43, 2131-2136.

Herzke, D., Olsson, E., Posner, S., 2012. Perfluoroalkyl and polyfluoroalkyl substances (PFASs) in consumer products in Norway - a pilot study. Chemosphere 88, 980-987.

Hickey, N. J., Crump, D., Jones, S.P., Kennedy, S.W., 2009. Effects of 18 perfluoroalkyl compounds on mrna expression in chicken embryo hepatocyte cultures. Toxicol Sci 111(2): 311-320.

Hinderliter, P.M., Mylchreest, E., Gannon, S.A., Butenhoff, J.L., Kennedy, G.L., Jr., 2005. Perfluorooctanoate: Placental and lactational transport pharmacokinetics in rats. Toxicology 211, 139-148.

Hoang, K.C., Edris, A., Su, J., Mukai, D.S., Mahon, S., Petrov, A.D., Kern, M., Ashan, C., Chen, Z., Tromberg, B.J., Narula, J., Brenner, M., 2009. Use of an oxygen-carrying blood substitute to improve intravascular optical coherence tomography imaging. Journal of biomedical optics 14, 034028.

Hoffman, K., Webster, T.F., Weisskopf, M.G., Weinberg, J., Vieira, V.M., 2010. Exposure to polyfluoroalkyl chemicals and attention deficit/hyperactivity disorder in U.S. children 12-15 years of age. Environ Health Perspect 118, 1762-1767.

Hoke, R.A., Bouchelle, L.D., Ferrell, B.D., Buck, R.C., 2012. Comparative acute freshwater hazard assessment and preliminary PNEC development for eight fluorinated acids. Chemosphere 87, 725-733.

Hu, W., Jones, P.D., Upham, B.L., Trosko, J.E., Lau, C., Giesy, J.P., 2002. Inhibition of Gap Junctional Intercellular Communication by Perfluorinated Compounds in Rat Liver and Dolphin Kidney Epithelial Cell Lines in Vitro and Sprague-Dawley Rats in Vivo. Toxicol Sci 68, 429-436.

Huber, S., Haug, L.S., Schlabach, M., 2011. Per- and polyfluorinated compounds in house dust and indoor air from northern Norway - A pilot study. Chemosphere 84, 1686-1693.

Iniguez, M. A., De Lecea, L., Guadano-Ferraz, A., Morte, B., Gerendasy, D., Sutcliffe, J.G., Bernal, J., 1996. Cell-specific effects of thyroid hormone on rc3/neurogranin expression in rat brain. Endocrinology 137(3): 1032-1041.

Iniguez, M. A., Rodriguez-Pena, A., Ibarrola, N., Aguilera, M., Muñoz, A., Bernal, J., 1993. Thyroid hormone regulation of rc3, a brain-specific gene encoding a protein kinase-c substrate. Endocrinology 133(2): 467-473.

Inoue, K., Okada, F., Ito, R., Kato, S., Sasaki, S., Nakajima, S., Uno, A., Saijo, Y., Sata, F., Yoshimura, Y., Kishi, R., Nakazawa, H., 2004. Perfluorooctane sulfonate (PFOS) and related perfluorinated compounds in human maternal and cord blood samples: assessment of PFOS exposure in a susceptible population during pregnancy. Environ Health Perspect 112, 1204-1207. 
Intrasuksri, U., Feller, D.R., 1991. Comparison of the effects of selected monocarboxylic, dicarboxylic and perfluorinated fatty acids on peroxisome proliferation in primary cultured rat hepatocytes. Biochem Pharmacol 42, 184-188.

Intrasuksri, U., Rangwala, S.M., O’Brien, M., Noonan, D.J., Feller, D.R., 1998. Mechanisms of peroxisome proliferation by perfluorooctanoic acid and endogenous fatty acids. General pharmacology 31, 187-197.

Ishibashi, H., Ishida, H., Matsuoka, M., Tominaga, N., Arizono, K., 2007. Estrogenic Effects of Fluorotelomer Alcohols for Human Estrogen Receptor Isoforms alpha and beta in Vitro. Biological and Pharmaceutical Bulletin 30, 1358-1359.

Ishibashi, H., Yamauchi, R., Matsuoka, M., Kim, J.-W., Hirano, M., Yamaguchi, A., Tominaga, N., Arizono, K., 2008. Fluorotelomer alcohols induce hepatic vitellogenin through activation of the estrogen receptor in male medaka (Oryzias latipes). Chemosphere 71, 1853-1859.

Jensen, M.S., Norgaard-Pedersen, B., Toft, G., Hougaard, D.M., Bonde, J.P., Cohen, A., Thulstrup, A.M., Ivell, R., Anand-Ivell, R., Lindh, C.H., Jonsson, B.A., 2012. Phthalates and perfluorooctanesulfonic acid in human amniotic fluid: temporal trends and timing of amniocentesis in pregnancy. Environ Health Perspect 120, 897-903.

Ji, K., Kim, S., Kho, Y., Paek, D., Sakong, J., Ha, J., Choi, K., 2012. Serum concentrations of major perfluorinated compounds among the general population in Korea: dietary sources and potential impact on thyroid hormones. Environ Int 45, 78-85.

Joensen, U.N., Bossi, R., Leffers, H., Jensen, A.A., Skakkebaek, N.E., Jorgensen, N., 2009. Do perfluoroalkyl compounds impair human semen quality? Environ Health Perspect 117, 923-927.

Johansson, N., Fredriksson, A., Eriksson, P., 2008. Neonatal exposure to perfluorooctane sulfonate (PFOS) and perfluorooctanoic acid (PFOA) causes neurobehavioural defects in adult mice. Neurotoxicology 29, 160-169.

Jones, P.D., Hu, W., De Coen, W., Newsted, J.L., Giesy, J.P., 2003. Binding of perfluorinated fatty acids to serum proteins. Environ Toxicol Chem 22, 2639-2649.

Karrman, A., Ericson, I., van Bavel, B., Darnerud, P.O., Aune, M., Glynn, A., Lignell, S., Lindstrom, G., 2007a. Exposure of perfluorinated chemicals through lactation: levels of matched human milk and serum and a temporal trend, 1996-2004, in Sweden. Environ Health Perspect 115, 226-230.

Karrman, A., Langlois, I., van Bavel, B., Lindstrom, G., Oehme, M., 2007b. Identification and pattern of perfluorooctane sulfonate (PFOS) isomers in human serum and plasma. Environ Int 33, 782-788.

Karrman, A., van Bavel, B., Jarnberg, U., Hardell, L., Lindstrom, G., 2006. Perfluorinated chemicals in relation to other persistent organic pollutants in human blood. Chemosphere 64, 1582-1591.

Kato, K., Wong, L.Y., Jia, L.T., Kuklenyik, Z., Calafat, A.M., 2011. Trends in exposure to polyfluoroalkyl chemicals in the U.S. Population: 1999-2008. Environ Sci Technol 45, 8037-8045.

Kawashima, Y., Kobayashi, H., Miura, H., Kozuka, H., 1995. Characterization of hepatic responses of rat to administration of perfluorooctanoic and perfluorodecanoic acids at low levels. Toxicology 99, 169-178.

Kennedy, G.L., Jr., Butenhoff, J.L., Olsen, G.W., O'Connor, J.C., Seacat, A.M., Perkins, R.G., Biegel, L.B., Murphy, S.R., Farrar, D.G., 2004. The toxicology of perfluorooctanoate. Crit Rev Toxicol 34, 351-384.

Kim, S., Choi, K., Ji, K., Seo, J., Kho, Y., Park, J., Park, S., Hwang, I., Jeon, J., Yang, H., Giesy, J.P., 2011. Trans-placental transfer of thirteen perfluorinated compounds and relations with fetal thyroid hormones. Environ Sci Technol 45, 7465-7472. 
Kleszczyński, K., Gardzielewski, P., Mulkiewicz, E., Stepnowski, P., Składanowski, A.C., 2007. Analysis of structure-cytotoxicity in vitro relationship (SAR) for perfluorinated carboxylic acids. Toxicology in Vitro 21, 1206-1211.

Kleszczyński, K., Składanowski, A.C., 2009. Mechanism of cytotoxic action of perfluorinated acids.: I. Alteration in plasma membrane potential and intracellular pH level. Toxicology and Applied Pharmacology 234, 300-305.

Knox, S.S., Jackson, T., Frisbee, S.J., Javins, B., Ducatman, A.M., 2011a. Perfluorocarbon exposure, gender and thyroid function in the C8 Health Project. J Toxicol Sci 36, 403-410.

Knox, S.S., Jackson, T., Javins, B., Frisbee, S.J., Shankar, A., Ducatman, A.M., 2011 b. Implications of early menopause in women exposed to perfluorocarbons. J Clin Endocrinol Metab 96, 1747-1753.

Kudo, N., Iwase, Y., Okayachi, H., Yamakawa, Y., Kawashima, Y., 2005. Induction of hepatic peroxisome proliferation by 8-2 telomer alcohol feeding in mice: formation of perfluorooctanoic acid in the liver. Toxicol Sci 86, 231-238.

Ladics, G.S., Kennedy, G.L., O'Connor, J., Everds, N., Malley, L.A., Frame, S.R., Gannon, S., Jung, R., Roth, T., Iwai, H., Shin-Ya, S., 2008. 90-day oral gavage toxicity study of 8 2 fluorotelomer alcohol in rats. Drug Chem Toxicol 31, 189-216.

Latała, A., Nędzi, M., Stepnowski, P., 2009. Acute toxicity assessment of perfluorinated carboxylic acids towards the Baltic microalgae. Environmental Toxicology and Pharmacology 28, 167-171.

Lau, C., 2012. Perfluoroalkyl acids: Recent research highlights. Reprod Toxicol 33, 405-409.

Lau, C., Anitole, K., Hodes, C., Lai, D., Pfahles-Hutchens, A., Seed, J., 2007.

Perfluoroalkyl acids: a review of monitoring and toxicological findings. Toxicol Sci 99, 366-394.

Lau, C., Butenhoff, J.L., Rogers, J.M., 2004. The developmental toxicity of perfluoroalkyl acids and their derivatives. Toxicol Appl Pharmacol 198, 231-241.

Lau, C., Thibodeaux, J.R., Hanson, R.G., Narotsky, M.G., Rogers, J.M., Lindstrom, A.B., Strynar, M.J., 2006. Effects of perfluorooctanoic acid exposure during pregnancy in the mouse. Toxicol Sci 90, 510-518.

Lau, C., Thibodeaux, J.R., Hanson, R.G., Rogers, J.M., Grey, B.E., Stanton, M.E., Butenhoff, J.L., Stevenson, L.A., 2003. Exposure to perfluorooctane sulfonate during pregnancy in rat and mouse. II: postnatal evaluation. Toxicol Sci 74, 382-392.

Lee, H., Mabury, S.A., 2011. A pilot survey of legacy and current commercial fluorinated chemicals in human sera from United States donors in 2009. Environmental science \& technology 45, 8067-8074.

Lehmler, H.-J., 2005. Synthesis of environmentally relevant fluorinated surfactants-a review. Chemosphere 58, 1471-1496.

Lieder, P.H., Chang, S.C., York, R.G., Butenhoff, J.L., 2009a. Toxicological evaluation of potassium perfluorobutanesulfonate in a 90-day oral gavage study with SpragueDawley rats. Toxicology 255, 45-52.

Lieder, P.H., York, R.G., Hakes, D.C., Chang, S.C., Butenhoff, J.L., 2009b. A twogeneration oral gavage reproduction study with potassium perfluorobutanesulfonate (K+PFBS) in Sprague Dawley rats. Toxicology 259, 33-45.

Lin, C.Y., Chen, P.C., Lin, Y.C., Lin, L.Y., 2009. Association among serum perfluoroalkyl chemicals, glucose homeostasis, and metabolic syndrome in adolescents and adults. Diabetes care 32, 702-707.

Lindeman, B., Maass, C., Duale, N., Gutzkow, K.B., Brunborg, G., Andreassen, A., 2012. Effects of per-and polyfluorinated compounds on adult rat testicular cells following in vitro exposure. Reprod Toxicol 33, 531-537. 
Lindh, C.H., Rylander, L., Toft, G., Axmon, A., Rignell-Hydbom, A., Giwercman, A., Pedersen, H.S., Goalczyk, K., Ludwicki, J.K., Zvyezday, V., Vermeulen, R., Lenters, V., Heederik, D., Bonde, J.P., Jonsson, B.A., 2012. Blood serum concentrations of perfluorinated compounds in men from Greenlandic Inuit and European populations. Chemosphere 88, 1269-1275.

Liu, W., Chen, S., Quan, X., Jin, Y.-H., 2008. Toxic effect of serial perfluorosulfonic and perfluorocarboxylic acids on the membrane system of a freshwater alga measured by flow cytometry. Environ. Toxicol. Chem. 27(7): 1597-1604.

Liu, C., Deng, J., Yu, L., Ramesh, M., Zhou, B., 2010a. Endocrine disruption and reproductive impairment in zebrafish by exposure to 8:2 fluorotelomer alcohol. Aquatic Toxicology 96, 70-76.

Liu, C., Yu, L., Deng, J., Lam, P.K.S., Wu, R.S.S., Zhou, B., 2009. Waterborne exposure to fluorotelomer alcohol 6:2 FTOH alters plasma sex hormone and gene transcription in the hypothalamic-pituitary-gonadal (HPG) axis of zebrafish. Aquatic Toxicology 93, 131-137.

Liu, C., Zhang, X., Chang, H., Jones, P., Wiseman, S., Naile, J., Hecker, M., Giesy, J.P., Zhou, B., 2010b. Effects of fluorotelomer alcohol 8:2 FTOH on steroidogenesis in H295R cells: targeting the cAMP signalling cascade. Toxicol Appl Pharmacol 247, 222-228.

Long, M., Bossi, R., Bonefeld-Jorgensen, E.C., 2012. Level and temporal trend of perfluoroalkyl acids in Greenlandic Inuit. Int J Circumpolar Health 71, 17998.

Lopez-Espinosa, M.J., Mondal, D., Armstrong, B., Bloom, M.S., Fletcher, T., 2012. Thyroid function and perfluoroalkyl acids in children living near a chemical plant. Environ Health Perspect 120, 1036-1041.

Loveless, S.E., Slezak, B., Serex, T., Lewis, J., Mukerji, P., O’Connor, J.C., Donner, E.M., Frame, S.R., Korzeniowski, S.H., Buck, R.C., 2009. Toxicological evaluation of sodium perfluorohexanoate. Toxicology 264, 32-44.

Luebker, D.J., Case, M.T., York, R.G., Moore, J.A., Hansen, K.J., Butenhoff, J.L., 2005a. Two-generation reproduction and cross-foster studies of perfluorooctanesulfonate (PFOS) in rats. Toxicology 215, 126-148.

Luebker, D.J., York, R.G., Hansen, K.J., Moore, J.A., Butenhoff, J.L., 2005b. Neonatal mortality from in utero exposure to perfluorooctanesulfonate (PFOS) in SpragueDawley rats: dose-response, and biochemical and pharamacokinetic parameters. Toxicology 215, 149-169.

Lundin, J.I., Alexander, B.H., Olsen, G.W., Church, T.R., 2009. Ammonium perfluorooctanoate production and occupational mortality. Epidemiology 20, 921-928.

MacNeil, J., Steenland, N.K., Shankar, A., Ducatman, A., 2009. A cross-sectional analysis of type II diabetes in a community with exposure to perfluorooctanoic acid (PFOA). Environ Res 109, 997-1003.

Maisonet, M., Terrell, M.L., McGeehin, M.A., Christensen, K.Y., Holmes, A., Calafat, A.M., Marcus, M., 2012. Maternal Concentrations of Polyfluoroalkyl Compounds during Pregnancy and Fetal and Postnatal Growth in British Girls. Environ Health Perspect 120, 1432-1437.

Malinverno, G., Pantini, G., Bootman, J., 1996. Safety evaluation of perfluoropolyethers, liquid polymers used in barrier creams and other skin-care products. Food Chem Toxicol 34, 639-650.

Maras, M., Vanparys, C., Muylle, F., Robbens, J., Berger, U., Barber, J.L., Blust, R., De Coen, W., 2006. Estrogen-like properties of fluorotelomer alcohols as revealed by mcf-7 breast cancer cell proliferation. Environ Health Perspect 114, 100-105.

Mariussen, E., 2012. Neurotoxic effects of perfluoroalkylated compounds: mechanisms of action and environmental relevance. Archives of toxicology 86, 1349-1367. 
Martin, J.W., Mabury, S.A., O’Brien, P.J., 2005. Metabolic products and pathways of fluorotelomer alcohols in isolated rat hepatocytes. Chemico-Biological Interactions 155, 165-180.

Melzer, D., Rice, N., Depledge, M.H., Henley, W.E., Galloway, T.S., 2010. Association between serum perfluorooctanoic acid (PFOA) and thyroid disease in the U.S. National Health and Nutrition Examination Survey. Environ Health Perspect 118, 686-692.

Mertens, J.J., Sved, D.W., Marit, G.B., Myers, N.R., Stetson, P.L., Murphy, S.R., Schmit, B., Shinohara, M., Farr, C.H., 2010. Subchronic toxicity of S-111-S-WB in Sprague Dawley rats. Int J Toxicol 29, 358-371.

Mitchell, R.J., Myers, A.L., Mabury, S.A., Solomon, K.R., Sibley, P.K., 2011. Toxicity of fluorotelomer carboxylic acids to the algae Pseudokirchneriella subcapitata and Chlorella vulgaris, and the amphipod Hyalella azteca. Ecotoxicology and Environmental Safety 74, 2260-2267.

Moe, M.K., Huber, S., Svenson, J., Hagenaars, A., Pabon, M., Trümper, M., Berger, U., Knapen, D., Herzke, D., 2012. The structure of the fire fighting foam surfactant Forafac $₫ 1157$ and its biological and photolytic transformation products. Chemosphere 89, 869-875.

Monroy, R., Morrison, K., Teo, K., Atkinson, S., Kubwabo, C., Stewart, B., Foster, W.G., 2008. Serum levels of perfluoroalkyl compounds in human maternal and umbilical cord blood samples. Environ Res 108, 56-62.

Mulkiewicz, E., Jastorff, B., Składanowski, A.C., Kleszczyński, K., Stepnowski, P., 2007. Evaluation of the acute toxicity of perfluorinated carboxylic acids using eukaryotic cell lines, bacteria and enzymatic assays. Environmental Toxicology and Pharmacology 23, 279-285.

Mundt, D.J., Mundt, K.A., Luippold, R.S., Schmidt, M.D., Farr, C.H., 2007. Clinical epidemiological study of employees exposed to surfactant blend containing perfluorononanoic acid. Occup Environ Med 64, 589-594.

Mylchreest, E., Ladics, G.S., Munley, S.M., Buck, R.C., Stadler, J.C., 2005a. Evaluation of the reproductive and developmental toxicity of a fluoroalkylethanol mixture. Drug Chem Toxicol 28, 159-175.

Mylchreest, E., Munley, S.M., Kennedy, G.L., Jr., 2005b. Evaluation of the developmental toxicity of 8-2 telomer B alcohol. Drug Chem Toxicol 28, 315-328.

Needham, L.L., Grandjean, P., Heinzow, B., Jørgensen, P.J., Nielsen, F., Patterson, D.G., Sjödin, A., Turner, W.E., Weihe, P., 2010. Partition of Environmental Chemicals between Maternal and Fetal Blood and Tissues. Environ Sci Technol 45, 1121-1126.

Nelson, J.W., Hatch, E.E., Webster, T.F., 2010. Exposure to polyfluoroalkyl chemicals and cholesterol, body weight, and insulin resistance in the general U.S. population. Environ Health Perspect 118, 197-202.

Newsted, J. L., S. Beach, Gallagher, S.P., Giesy, J.P., 2008. Acute and chronic effects of perfluorobutane sulfonate (pfbs) on the mallard and northern bobwhite quail. Arch Environ Contam Toxicol 54(3): 535-545.

Nilsson, H., Karrman, A., Rotander, A., van Bavel, B., Lindstrom, G., Westberg, H., 2010a. Inhalation exposure to fluorotelomer alcohols yield perfluorocarboxylates in human blood? Environ Sci Technol 44, 7717-7722.

Nilsson, H., Karrman, A., Westberg, H., Rotander, A., van Bavel, B., Lindstrom, G., $2010 \mathrm{~b}$. A time trend study of significantly elevated perfluorocarboxylate levels in humans after using fluorinated ski wax. Environ Sci Technol 44, 2150-2155.

Nilsson, S., Makela, S., Treuter, E., Tujague, M., Thomsen, J., Andersson, G., Enmark, E., Pettersson, K., Warner, M., Gustafsson, J.A., 2001. Mechanisms of estrogen action. Physiol Rev 81, 1535-1565. 
Nobels, I., Dardenne, F., Coen, W.D., Blust, R., 2010. Application of a multiple endpoint bacterial reporter assay to evaluate toxicological relevant endpoints of perfluorinated compounds with different functional groups and varying chain length. Toxicology in Vitro 24, 1768-1774.

Nøst, T. H., Helgason, L.B., Harju, M., Heimstad, E. S., Gabrielsen, G. W., Jenssen, B. M., 2012. Halogenated organic contaminants and their correlations with circulating thyroid hormones in developing arctic seabirds. Sci Total Environ 414(0): 248-256.

O’Brien, J.M., Crump, D., Mundy, L.J., Chu, S., McLaren, K.K., Vongphachan, V., Letcher, R.J., Kennedy, S.W., 2009. Pipping success and liver mRNA expression in chicken embryos exposed in ovo to C8 and C11 perfluorinated carboxylic acids and C10 perfluorinated sulfonate. Toxicology Letters 190, 134-139.

Oda, Y., Nakayama, S., Harada, K., Koizumi, A., 2007. Negative results of umu genotoxicity test of fluorotelomer alcohols and perfluorinated alkyl acids. Environmental Health and Preventive Medicine 12, 217-219.

Okada, E., Sasaki, S., Saijo, Y., Washino, N., Miyashita, C., Kobayashi, S., Konishi, K., Ito, Y.M., Ito, R., Nakata, A., Iwasaki, Y., Saito, K., Nakazawa, H., Kishi, R., 2012. Prenatal exposure to perfluorinated chemicals and relationship with allergies and infectious diseases in infants. Environ Res 112, 118-125.

Olsen, G.W., Burris, J.M., Mandel, J.H., Zobel, L.R., 1999. Serum perfluorooctane sulfonate and hepatic and lipid clinical chemistry tests in fluorochemical production employees. Journal of occupational and environmental medicine / American College of Occupational and Environmental Medicine 41, 799-806.

Olsen, G.W., Chang, S.C., Noker, P.E., Gorman, G.S., Ehresman, D.J., Lieder, P.H., Butenhoff, J.L., 2009. A comparison of the pharmacokinetics of perfluorobutanesulfonate (PFBS) in rats, monkeys, and humans. Toxicology 256, 65-74.

Olsen, G.W., Gilliland, F.D., Burlew, M.M., Burris, J.M., Mandel, J.S., Mandel, J.H., 1998. An epidemiologic investigation of reproductive hormones in men with occupational exposure to perfluorooctanoic acid. Journal of occupational and environmental medicine / American College of Occupational and Environmental Medicine 40, 614-622.

Olsen, G.W., Zobel, L.R., 2007. Assessment of lipid, hepatic, and thyroid parameters with serum perfluorooctanoate (PFOA) concentrations in fluorochemical production workers. International archives of occupational and environmental health 81, 231-246.

Permadi, H., Lundgren, B., Andersson, K., DePierre, J.W., 1992. Effects of perfluoro fatty acids on xenobiotic-metabolizing enzymes, enzymes which detoxify reactive forms of oxygen and lipid peroxidation in mouse liver. Biochem Pharmacol 44, 1183-1191.

Pinkas, A., Slotkin, T.A., Brick-Turin, Y., Van der Zee, E.A., Yanai, J., 2010. Neurobehavioral teratogenicity of perfluorinated alkyls in an avian model. Neurotoxicol Teratol 32, 182-186.

Pistocchi, A., Loos, R., 2009. A map of European emissions and concentrations of PFOS and PFOA. Environmental Science and Technology 43, 9237-9244.

Prevedouros, K., Cousins, I.T., Buck, R.C., Korzeniowski, S.H., 2006. Sources, fate and transport of perfluorocarboxylates. Environ Sci Technol 40, 32-44.

Rand, A.A., Mabury, S.A., 2012. Assessing the structure-activity relationships of fluorotelomer unsaturated acids and aldehydes with glutathione Reactivity of glutathione with fluorotelomer unsaturated acids and aldehydes. Cell Biol Toxicol 28, 115-124.

Raymer, J.H., Michael, L.C., Studabaker, W.B., Olsen, G.W., Sloan, C.S., Wilcosky, T., Walmer, D.K., 2012. Concentrations of perfluorooctane sulfonate (PFOS) and perfluorooctanoate (PFOA) and their associations with human semen quality measurements. Reprod Toxicol 33, 419-427. 
Riess, J.G., 2002. Blood substitutes and other potential biomedical applications of fluorinated colloids. Journal of Fluorine Chemistry 114, 119-126.

Riess, J.G., Krafft, M.P., 1998. Fluorinated materials for in vivo oxygen transport (blood substitutes), diagnosis and drug delivery. Biomaterials 19, 1529-1539.

Rosal, R., Rodea-Palomares, I., Boltes, K., Fernández-Piñas, F., Leganés, F., Petreb, A., 2010. Ecotoxicological assessment of surfactants in the aquatic environment: Combined toxicity of docusate sodium with chlorinated pollutants. Chemosphere 81 (2): 288-293.

Rosenmai, A.K., Nielsen, F.K., Pedersen, M., Hadrup, N., Trier, X., Christensen, J.H., Vinggaard, A.M., 2012. Fluorochemicals used in food packaging inhibit male sex hormone synthesis. Toxicol Appl Pharmacol.

Rylander, C., Brustad, M., Falk, H., Sandanger, T.M., 2009. Dietary predictors and plasma concentrations of perfluorinated compounds in a coastal population from northern Norway. J Environ Public Health 2009, 268219.

Sakr, C.J., Leonard, R.C., Kreckmann, K.H., Slade, M.D., Cullen, M.R., 2007. Longitudinal study of serum lipids and liver enzymes in workers with occupational exposure to ammonium perfluorooctanoate. Journal of occupational and environmental medicine / American College of Occupational and Environmental Medicine 49, 872-879.

Savitz, D.A., Stein, C.R., Elston, B., Wellenius, G.A., Bartell, S.M., Shin, H.M., Vieira, V.M., Fletcher, T., 2012. Relationship of perfluorooctanoic Acid exposure to pregnancy outcome based on birth records in the mid-ohio valley. Environ Health Perspect 120, 1201-1207.

Shankar, A., Xiao, J., Ducatman, A., 2012. Perfluorooctanoic Acid and Cardiovascular Disease in US Adults. Arch Intern Med, 1-7.

Shi, Z., Ding, L., Zhang, H., Feng, Y., Xu, M., Dai, J., 2009. Chronic exposure to perfluorododecanoic acid disrupts testicular steroidogenesis and the expression of related genes in male rats. Toxicol Lett 188, 192-200.

Shi, Z., Zhang, H., Liu, Y., Xu, M., Dai, J., 2007. Alterations in gene expression and testosterone synthesis in the testes of male rats exposed to perfluorododecanoic acid. Toxicol Sci 98, 206-215.

Sibinski, L.J., 1987. Final report of a two year oral (diet) toxicity and carcinogenicity study of fluorochemical FC-143 (perfluorooctanane ammonium carboxylate) in rats. 3M Company/RIKER Exp No 0281CR0012; 8EHQ-1087-0394 1-4.

Sinclair, E., Kim, S.K., Akinleye, H.B., Kannan, K., 2007. Quantitation of gas-phase perfluoroalkyl surfactants and fluorotelomer alcohols released from nonstick cookware and microwave popcorn bags. Environ Sci Technol 41, 1180-1185.

Slotkin, T.A., MacKillop, E.A., Melnick, R.L., Thayer, K.A., Seidler. F.J., 2008. Developmental neurotoxicity of perfluorinated chemicals modeled in vitro. Environ Health Perspect 116(6): 716-722.

So, M.K., Yamashita, N., Taniyasu, S., Jiang, Q., Giesy, J.P., Chen, K., Lam, P.K., 2006. Health risks in infants associated with exposure to perfluorinated compounds in human breast milk from Zhoushan, China. Environ Sci Technol 40, 2924-2929.

Steenland, K., Fletcher, T., Savitz, D.A., 2010a. Epidemiologic evidence on the health effects of perfluorooctanoic acid (PFOA). Environ Health Perspect 118, 1100-1108.

Steenland, K., Tinker, S., Frisbee, S., Ducatman, A., Vaccarino, V., 2009. Association of perfluorooctanoic acid and perfluorooctane sulfonate with serum lipids among adults living near a chemical plant. Am J Epidemiol 170, 1268-1278.

Steenland, K., Tinker, S., Shankar, A., Ducatman, A., 2010b. Association of perfluorooctanoic acid (PFOA) and perfluorooctane sulfonate (PFOS) with uric acid among adults with elevated community exposure to PFOA. Environ Health Perspect 118, 229-233. 
Stein, C.R., Savitz, D.A., 2011. Serum perfluorinated compound concentration and attention deficit/hyperactivity disorder in children 5-18 years of age. Environ Health Perspect 119, 1466-1471.

Stein, C.R., Wolff, M.S., Calafat, A.M., Kato, K., Engel, S.M., 2012. Comparison of polyfluoroalkyl compound concentrations in maternal serum and amniotic fluid: $A$ pilot study. Reprod Toxicol 34, 312-316.

Takagi, A., Sai, K., Umemura, T., Hasegawa, R., Kurokawa, Y., 1991. Short-term exposure to the peroxisome proliferators, perfluorooctanoic acid and perfluorodecanoic acid, causes significant increase of 8-hydroxydeoxyguanosine in liver DNA of rats. Cancer Lett 57, 55-60.

Takagi, A., Sai, K., Umemura, T., Hasegawa, R., Kurokawa, Y., 1992. Hepatomegaly is an early biomarker for hepatocarcinogenesis induced by peroxisome proliferators. Environ Pathol Toxicol Oncol 11, 145-149.

Thibodeaux, J.R., Hanson, R.G., Rogers, J.M., Grey, B.E., Barbee, B.D., Richards, J.H., Butenhoff, J.L., Stevenson, L.A., Lau, C., 2003. Exposure to perfluorooctane sulfonate during pregnancy in rat and mouse. I: maternal and prenatal evaluations. Toxicol Sci 74, 369-381.

Tiido, T., Rignell-Hydbom, A., Jonsson, B.A., Giwercman, Y.L., Pedersen, H.S., Wojtyniak, B., Ludwicki, J.K., Lesovoy, V., Zvyezday, V., Spano, M., Manicardi, G.C., Bizzaro, D., Bonefeld-Jorgensen, E.C., Toft, G., Bonde, J.P., Rylander, L., Hagmar, L., Giwercman, A., 2006. Impact of PCB and p, $p^{\prime}-D D E$ contaminants on human sperm $Y: X$ chromosome ratio: studies in three European populations and the Inuit population in Greenland. Environ Health Perspect 114, 718-724.

Toft, G., Jonsson, B.A., Lindh, C.H., Giwercman, A., Spano, M., Heederik, D., Lenters, V., Vermeulen, R., Rylander, L., Pedersen, H.S., Ludwicki, J.K., Zviezdai, V., Bonde, J.P., 2012. Exposure to perfluorinated compounds and human semen quality in arctic and European populations. Hum Reprod 27, 2532-2540.

Trudel, D., Horowitz, L., Wormuth, M., Scheringer, M., Cousins, I.T., Hungerbühler, K., 2008. Estimating Consumer Exposure to PFOS and PFOA. Risk Analysis 28, 251-269.

Upham, B.L., Park, J.S., Babica, P., Sovadinova, I., Rummel, A.M., Trosko, J.E., Hirose, A., Hasegawa, R., Kanno, J., Sai, K., 2009. Structure-activity-dependent regulation of cell communication by perfluorinated fatty acids using in vivo and in vitro model systems. Environ Health Perspect 117, 545-551.

Vanden Heuvel, J.P., Kuslikis, B.I., Shrago, E., Peterson, R.E., 1991. Inhibition of longchain acyl-CoA synthetase by the peroxisome proliferator perfluorodecanoic acid in rat hepatocytes. Biochem Pharmacol 42, 295-302.

Vanparys, C., Maras, M., Lenjou, M., Robbens, J., Van Bockstaele, D., Blust, R., De Coen, W., 2006. Flow cytometric cell cycle analysis allows for rapid screening of estrogenicity in MCF-7 breast cancer cells. Toxicology in Vitro 20, 1238-1248.

Vestergaard, S., Nielsen, F., Andersson, A.M., Hjollund, N.H., Grandjean, P., Andersen, H.R., Jensen, T.K., 2012. Association between perfluorinated compounds and time to pregnancy in a prospective cohort of Danish couples attempting to conceive. Hum Reprod 27, 873-880.

Vongphachan, V., Cassone, C.G., Wu, D., Chiu, S., Crump, D., Kennedy, S.W., 2011. Effects of perfluoroalkyl compounds (PFCs) on mRNA expression levels of thyroid hormone-responsive genes in primary cultures of avian neuronal cells. Toxicol Sci. 120 (2): 392-402.

Walters, A., Santillo, D., 2006. Uses of perfluorinated substances. Greenpeace. 
Wang, C., Wang, T., Liu, W., Ruan, T., Zhou, Q., Liu, J., Zhang, A., Zhao, B., Jiang, G., 2012. The in vitro estrogenic activities of polyfluorinated iodine alkanes. Environ Health Perspect 120, 119-125.

Wang, I.J., Hsieh, W.S., Chen, C.Y., Fletcher, T., Lien, G.W., Chiang, H.L., Chiang, C.F., $\mathrm{Wu}, \mathrm{T} . \mathrm{N} ., \mathrm{C}$-n, P.C., 2011. The effect of prenatal perfluorinated chemicals exposures on pediatric atopy. Environ Res 111, 785-791.

Wang, Z., Ud-Daula, A., Fiedler, S., Schramm, K.-W., 2010. Impact of fluorotelomer alcohols (FTOH) on the molecular and macroscopic phenotype of Tetrahymena thermophila. Environ Sci Pollut Res 17, 154-164.

Wania, F., 2006. Potential of degradable organic chemicals for absolute and relative enrichment in the arctic. Environ Sci Technol 40, 569-577.

Wania, F., Mackay, D., 1996. Tracking the distribution of persistent organic pollutants. Environ Sci Technol 30, A390-A396.

Washburn, S.T., Bingman, T.S., Braithwaite, S.K., Buck, R.C., Buxton, L.W., Clewell, H.J., Haroun, L.A., Kester, J.E., Rickard, R.W., Shipp, A.M., 2005. Exposure assessment and risk characterization for perfluorooctanoate in selected consumer articles. Environ Sci Technol 39, 3904-3910.

Washino, N., Saijo, Y., Sasaki, S., Kato, S., Ban, S., Konishi, K., Ito, R., Nakata, A., Iwasaki, Y., Saito, K., Nakazawa, H., Kishi, R., 2009. Correlations between prenatal exposure to perfluorinated chemicals and reduced fetal growth. Environ Health Perspect 117, 660-667.

Weihe, P., Kato, K., Calafat, A.M., Nielsen, F., Wanigatunga, A.A., Needham, L.L., Grandjean, P., 2008. Serum concentrations of polyfluoroalkyl compounds in Faroese whale meat consumers. Environ Sci Technol 42, 6291-6295.

Weiss, J.M., Andersson, P.L., Lamoree, M.H., Leonards, P.E., van Leeuwen, S.P., Hamers, T., 2009. Competitive binding of poly- and perfluorinated compounds to the thyroid hormone transport protein transthyretin. Toxicol Sci 109, 206-216.

White, S.S., Calafat, A.M., Kuklenyik, Z., Villanueva, L., Zehr, R.D., Helfant, L., Strynar, M.J., Lindstrom, A.B., Thibodeaux, J.R., Wood, C., Fenton, S.E., 2007. Gestational PFOA exposure of mice is associated with altered mammary gland development in dams and female offspring. Toxicol Sci 96, 133-144.

Whitworth, K.W., Haug, L.S., Baird, D.D., Becher, G., Hoppin, J.A., Skjaerven, R., Thomsen, C., Eggesbo, M., Travlos, G., Wilson, R., Cupul-Uicab, L.A., Brantsaeter, A.L., Longnecker, M.P., 2012a. Perfluorinated Compounds in Relation to Birth Weight in the Norwegian Mother and Child Cohort Study. Am J Epidemiol 175, 1209-1216.

Whitworth, K.W., Haug, L.S., Baird, D.D., Becher, G., Hoppin, J.A., Skjaerven, R., Thomsen, C., Eggesbo, M., Travlos, G., Wilson, R., Longnecker, M.P., 2012b. Perfluorinated compounds and subfecundity in pregnant women. Epidemiology 23, 257-263.

Wolf, C.J., Fenton, S.E., Schmid, J.E., Calafat, A.M., Kuklenyik, Z., Bryant, X.A., Thibodeaux, J., Das, K.P., White, S.S., Lau, C.S., Abbott, B.D., 2007. Developmental toxicity of perfluorooctanoic acid in the CD-1 mouse after cross-foster and restricted gestational exposures. Toxicol Sci 95, 462-473.

Wolf, C.J., Schmid, J.E., Lau, C., Abbott, B.D., 2012. Activation of mouse and human peroxisome proliferator-activated receptor-alpha (PPARalpha) by perfluoroalkyl acids (PFAAs): further investigation of C4-C12 compounds. Reprod Toxicol 33, 546-551.

Wolf, C.J., Takacs, M.L., Schmid, J.E., Lau, C., Abbott, B.D., 2008. Activation of mouse and human peroxisome proliferator-activated receptor alpha by perfluoroalkyl acids of different functional groups and chain lengths. Toxicol Sci 106, 162-171. 
Wolf, C.J., Zehr, R.D., Schmid, J.E., Lau, C., Abbott, B.D., 2010. Developmental effects of perfluorononanoic Acid in the mouse are dependent on peroxisome proliferatoractivated receptor-alpha. PPAR research 2010.

Yang, Q., Abedi-Valugerdi, M., Xie, Y., Zhao, X.Y., Moller, G., Nelson, B.D., DePierre, J.W., 2002. Potent suppression of the adaptive immune response in mice upon dietary exposure to the potent peroxisome proliferator, perfluorooctanoic acid. Int Immunopharmacol 2, 389-397.

Yao, X., Zhong, L., 2005. Genotoxic risk and oxidative DNA damage in HepG2 cells exposed to perfluorooctanoic acid. Mutat Res 587, 38-44.

York, R.G., Kennedy, G.L., Jr., Olsen, G.W., Butenhoff, J.L., 2010. Male reproductive system parameters in a two-generation reproduction study of ammonium perfluorooctanoate in rats and human relevance. Toxicology 271, 64-72.

Zheng, X.M., Liu, H.L., Shi, W., Wei, S., Giesy, J.P., Yu, H.X., 2012. Effects of perfluorinated compounds on development of zebrafish embryos. Environ Sci Pollut Res 19, 2498-2505.

\section{Appendix A}

Banks, R. E., Smart, B.E., Tatlow, J.C. 1994. Organofluorine Chemistry: Principles and Commercial Applications. Plenum 1994.

Burns, D.C., Ellis, D.A., Li, H., McMurdo, C.J., Webster, E., 2008. Experimental pKa Determination for Perfluorooctanoic Acid (PFOA) and the Potential Impact of pKa Concentration Dependence on Laboratory-Measured Partitioning Phenomena and Environmental Modeling. Environ. Sci. Technol. 42, 9283-9288.

Cheng, J., Psillakis, E., Hoffmann, M.R. Colussi, A.J., 2009a. Acid Dissociation versus Molecular Association of Perfluoroalkyl Compounds: Environmental Implications. J. Phys. Chem. A 113, 8152-8156.

Cheng, J., Psillakis, E., Hoffmann, M.R. Colussi, A.J., 2009b. Acid Dissociation versus Molecular Association of Perfluoroalkyl Compounds: Environmental Implications. Correction. J. Phys. Chem. A 113, 9578.

D’Eon, J.C., Crozier, P.W., Furdui, V.I., Reiner, E.J., Libelo, E.L., Mabury, S.A., 2009. Perfluorinated phosphonic acids in Canadian surface waters and wastewater treat ment plant effluent: discovery of a new class of perfluorinated acids. Environ. Toxicol. Chem. 28, 2101-2107.

Ellis, D.A., Webster, E., 2009. Response to Comment on "Aerosol Enrichment of the Surfactant PFO and Mediation of the Water-Air Transport of Gaseous PFOA". Environ. Sci. Technol. 43, 1234-1235.

Goss, K.-U., 2008. The pKa Values of PFOA and Other Highly Fluorinated Carboxylic Acids. Environ. Sci. Technol. 42, 456-458.

Goss, K.-U., Arp, H.P.H., 2009. Comment on "Experimental pKa Determination for Perfluorooctanoic Acid (PFOA) and the Potential Impact of pKa Concentration Dependence on Laboratory-Measured Partitioning Phenomena and Environmental Modeling". Environ. Sci. Technol. 43, 5150-5151.

Kissa, E., 2001. Fluorinated surfactants and repellents. Surfactant Science Series, Marcel Dekker, New York, NY, 97, (Fluorinated Surfactants and Repellents (2nd Edition)), 1-615.

Rayne, S., Forest, K., Friesen, K.J., 2008. Congener-specific numbering systems for the environmentally relevant $C 4$ through C8 perfluorinated homologue groups of alkyl sulfonates, carboxylates, telomer alcohols, olefins, and acids, and their derivatives, J. Environ. Sci. Health A, 43, 1391-1401. 



\section{Sammanfattning}

Nordiska Kemikaliegruppen (NKG) som är underställd Nordiska Minister Rådet har via Klima- og forurensningsdirektoratet (KLIF) gett i uppdrag till författarna att genomföra en Nordisk studie baserad på öppna infomationskällor samt egna marknadsstudier för att beskriva de vanligaste perfluorerade ämnena (PFC) med mindre fokus på PFOS och PFOA.

Undersökningen omfattar tre delmoment:

1. Identifiering av relevanta per- och polyfluorerade ämnen och deras användning inom olika industrisektorer på den nordiska marknaden.

2. Förekomst i industri-och konsumentprodukter och potentiella utsläpp till och i den nordiska miljön och människor av de ämnen som beskrivs i delmoment 1 .

3. En sammanfattning av kunskapen om toxiska effekter på människa och miljö hos de ämnen som prioriterats i delmoment 2 .

Intervjuer genomfördes med fler än 50 aktörer på den Nordiska marknaden med syftet att få information om användning och typ av av PFCämnen. Denna undersökning gav emellertid magert resultat. Parallellt med denna kartläggning togs därför en nettolista över PFC-ämnen fram baserat på listor (var och en för sig och tillsammans ofullständiga) från OECD, REACH förregistreringsdatabas samt den nordiska SPINdatabasen. Större delen av varuproduktionen sker idag utanför EU och dagens regelverk inte ger tillräckliga förutsättningar för att få tillräcklig information om specifika PFC ämnen som finns i importerade varor. Denna nettolista är således inte komplett varför det kan finnas avsevärt fler PFC-ämnen som används på den nordiska marknaden.

Det finns få studier om PFC-ämnens förekomst i miljön i de nordiska länderna utöver PFOA och PFOS som omfattar både biotiska (luft, mark och vatten) och abiotiska (djur och människa) data.

De flesta humandata avseende PFCA och PFSA från åren 1992 till 2010 kommer från Norge och Sverige, färre från Danmark och inga uppgifter från Island och Finland. Gällande PFCA visar de flesta studier på förekomst av PFOA, PFHxA och PFNA. Även andra PFCA ämnen (C10C13) har också påvisats i flera studier. För PFSA är PFOS och PFHxS är 
de mest studerade föreningarna. Humandata saknas helt för PFAL, FTS, PAP/di-PAP samt FTMAPs.

I jämförelse med långkedjiga PFC-ämnen ( $\geq \mathrm{C} 8)$ är de kortkedjiga föreningarna bedömts vara mindre toxiska men ett antal studier visar på både ekotoxikologiska och humantoxikologiska effekter. Inom detta område är dock bristen på studier stor.

I stort iakttas minskande halter av PFOA och PFOS i miljön sedan 2002. Däremot observeras en ökande halt av kortkedjiga sulfonater i miljön. I jämförelse med andra länder är bakgrundskoncentrationen i miljön av PFOA och PFOS lägre i de nordiska länderna särskilt i jämförelse med centraleuropeiska länder, som kan förväntas pga lägre befolkningstäthet och mindre industriell verksamhet i de Nordiska länderna. . Dessa ämnen har även hittats i Arktis, långt från alla källor, vilket visar att dessa ämnen är globala föroreningar.

Ett resultat av denna översikt av förekomsten av fluorerande ämnen i miljön, är att det finns en stor informations- och kunskapsbrist om PFC utöver PFOA och PFOS. Dessutom finns generellt en stor brist på humanoch miljödata kring dessa PFC-ämnen. De få data som finns indikerar viss toxisk påverkan på människa och miljö. Det krävs fler och djupare studier för att få en tydligare bild av dessa PFC ämnens innan mer långtgående slutsatser kan dras om deras toxiska egenskaper.

Bristen på fysikalisk-kemiska data för PFC-ämnen utöver PFOA och PFOS utgör ett hinder för modellberäkningar kring dessa ämnens spridning i miljön

Bristen på analytiska referenssubstanser utgör idag också ett hinder för utökade studier kring dessa ämnens förekomst i människa och miljö. 


\title{
Appendix A - List of abbreviations and acronyms
}

\author{
\begin{tabular}{ll}
\hline Literature review terminology & OECD glossary \\
& 27
\end{tabular} \\ Perfluoro- / Perfluorinated \\ A general term for a substance where fluorine $(F)$ is substituted \\ for all hydrogen $(\mathrm{H})$ atoms attached to carbon atoms except \\ carbon atoms whose substitution would affect the nature of \\ the functional group(s) present ${ }^{2}$. Examples: $\mathrm{F}\left(\mathrm{CF}_{2}\right)_{n} \mathrm{CHO}$, \\ $\mathrm{F}\left(\mathrm{CF}_{2}\right)_{n} \mathrm{CO}_{2} \mathrm{H}, \mathrm{F}\left(\mathrm{CF}_{2}\right)_{n} \mathrm{SO}_{3} \mathrm{H},\left(\mathrm{CF}_{3}\right)_{2} \mathrm{NH}$ \\ A fully fluorinated or perfluorinated chemi- \\ cal is one in which all the carbon-hydrogen \\ bonds in a chain have been replaced by \\ carbon-fluorine ones. All fully fluorinated \\ chemicals are man-made. Examples include \\ perfluorooctanoic acid (PFOA) and perfluo- \\ rooctane sulfonate (PFOS).
}

\section{Perfluoroalkyl Substance / Compound (PFA)}

A general term for a substance that is perfluorinated according to the definition given above, but excluding perfluorocarbons.

Comments: The term has also been used to describe substances which contain a perfluoroalkyl moiety attached to other atoms that may not be perfluorinated but may have potential to transform to a perfluoroalkyl substance. Justification for the acronym PFA is given in Part 3 of this document.

\section{Perfluorocarbon (PFC)}

A perfluorinated hydrocarbon, especially a perfluorinated alkane, $\mathrm{C}_{n} \mathrm{~F}_{2 \mathrm{n}+2}$. Perfluorocarbons contain only carbon and fluorine atoms.
A substance which bears a perfluorocarbon, also known as a perfluororoalkyl, functional group. $F(C F 2) n-X$ where $n$ is an integer and $X$ is not a halogen, or hydrogen.
Perfluorinated chemicals in which all carbon-hydrogen bonds in a chain have been replaced by carbon-fluorine bonds. Examples include perfluorooctanoic acid (PFOA) and perfluorooctane sulfonate (PFOS). PFC term also refers to PFC precursors, chemicals which contain a perfluoroalkyl moiety attached to other atoms that may not be perfluorinated, and have potential to transform to produce PFCs.

Perfluorinated Surfactant / "Perfluorinated Tenside (PFT)" (in publications of German origin)

A general term for a surface active, low molecular weight $(<1,000$ daltons), substance where fluorine $(F)$ is substituted for all hydrogen $(\mathrm{H})$ atoms attached to carbon atoms except A term used to describe a surface active, low molecular weight $(<1,000)$, substance where all carbons bear fluorine in place of hydrogen; the term Fluorosurfactant is less carbon atoms whose substitution would affect the nature of specific but misused synonymously; a perfluorinated example is $\mathrm{F}(\mathrm{CF} 2) 6 \mathrm{SO} 3$ $\mathrm{NH} 4+$; while a fluorinated surfactant might be $\mathrm{F}(\mathrm{CF} 2) 4 \mathrm{CH} 2 \mathrm{SO} 2-\mathrm{NH} 4+$ 


\begin{tabular}{l} 
Literature review terminology \\
\hline Perfluoroalkyl Acid / Perfluorinated Acid (PFAA) \\
A general term for a substance which contains a perfluoroalkyl, \\
$\mathrm{F}\left(\mathrm{CF}_{2}\right)_{n}$-, functionality bound to an acid functionality, e.g., \\
carboxylate, sulfonate, phosphonate. \\
Perfluoroalkyl Carboxylic Acid / Perfluoroalkyl Carboxylate (PFCA) \\
A general term for a substance whose chemical structure is
\end{tabular}

A general term for a substance whose chemical structure is $\mathrm{F}\left(\mathrm{CF}_{2}\right)_{\mathrm{n}} \mathrm{CO}_{2} \mathrm{H}$ and its anionic form $\mathrm{F}\left(\mathrm{CF}_{2}\right)_{n} \mathrm{CO}_{2}$.

Comments: This term may also be used to describe the salts of these acids (e.g., ammonium, sodium, potassium). Justification for the acronyms proposed to represent the various species (free acid, anion and salts) is given in Part 3 of this document.

Examples:

PFNA:

Perfluorononanoic acid, $\mathrm{F}\left(\mathrm{CF}_{2}\right)_{8} \mathrm{CO}_{2} \mathrm{H}$, is a fully fluorinated, nine-carbon chain length carboxylic acid (C9) (CAS 375-95-1).

OECD glossary ${ }^{27}$

PFOA:

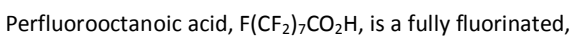
eight-carbon chain length carboxylic acid (C8) (CAS 335-67-1).

APFO:

Perfluoroalkyl acids

Perfuroakyl acids

Perfluorinated carboxylic acids and their salts are a series of substances whose anion has the general structure of CF3(CF2)nCOO-. Certain members of this class, including the PFCA with 8 carbons, called perfluorooctanoic acid (PFOA or C8), are manufactured as a processing aid to produce fluoropolymers.

Perfluorononanoic acid is a fully fluorinated, nine-carbon chain length carboxylic acid (C9) (CAS 375-95-1).

Perfluorooctanoic acid is a fully fluorinated, eight-carbon chain carboxylic acid (C8) (CAS 335-67-1) sometimes used to refer to the anionic salt form.

Perfluoroalkyl sulfonate is a generic term used to describe any fully fluorinated carbon chain length sulfonic acid, including higher and lower homologues as well as PFOS.

Comments: This term may also be used to describe its salts (e.g., ammonium, sodium, potassium). Justification for the acronyms proposed to represent the various species (free acid, anion and salts) is given in Part 3 of this document.

\section{Examples}

PFOS:

Perfluorooctane sulfonic acid (CAS 1763-23-1) / sulfonate (CAS 45298-90-6) is a fully fluorinated, eight-carbon chain homologue.

Perfluorooctane sulfonic acid is a fully fluorinated, eight chain sulfonic acid (CAS 1763-23-1) sometimes used to refer to the anionic salt form. 


\begin{tabular}{l} 
Literature review terminology \\
\hline PFHXS: \\
Perfluorohexane sulfonic acid (CAS 355-46-4) / sulfonate (CAS \\
108427-53-8) is a fully fluorinated, six-carbon chain homologue. \\
PFBS: \\
Perfluorobutane sulfonic acid (CAS 375-73-5) / sulfonate (CAS \\
$45187-15-3$ ) is a fully fluorinated, four-carbon chain homologue. \\
Perfluoroether \\
A general term for a substance which contains short perfluoroal- \\
kyl moieties, typically 1-3 carbon atoms, connected to an oxygen \\
and capped by the same type of perfluoroalkyl / perfluorocarbon \\
functionality or/and by other non-fluorinated functionality.
\end{tabular}

OECD glossary ${ }^{27}$

\section{Polyfluoroether}

A general term for a substance which contains short fluoroalkyl moieties that are not fully fluorinated and do contain hydrogen bound to carbon, typically 1-3 carbon atoms, connected to an oxygen and capped by a fluoroalkyl / fluorocarbon functionality or/and by other non-fluorinated functionality.

\section{Perfluoropolyether (PFPE)}

A general term for a substance which contains short perfluoroalkyl moieties, 1-3 carbon atoms, connected by oxygen bridges and capped by the same type of perfluoroalkyl / perfluorocarbon functionality or/and by other non-fluorinated functionality.

\section{ECF \& TELOMERS - TERMINOLOGY}

\section{Electrochemical fluorination}

A process technology used to manufacture fluorinated chemicals where an organic raw material is dissolved in hydrogen fluoride and electrolyzed, resulting in the replacement of hydrogen with fluorine The free-radical nature of the process leads to rearrangement resulting in a product mixture of linear and branched isomers of multiple carbon chain lengths.

Comment: A systematic numbering system for identifying the linear and branched congeners of several families of perfluoroalkyl substances has been proposed ${ }^{3}$.

\section{Telomerisation (or Telomerisation)}

A process technology used to manufacture fluorinated chemicals where pentafluoroethyl iodide (telogen) is reacted with tetrafluoroethylene (TFE, taxogen) to yield a mixture of even carbonnumbered perfluoroalkyl iodides $\mathrm{F}\left(\mathrm{CF}_{2} \mathrm{CF}_{2}\right)_{n}$ l.

Telomer (or Fluorotelomer)

A general term for a substance derived from a raw material produced from the telomerisation process.

Telomer Based Product: Chemical substances that have the fluoroalkyl portion of the molecule derived from telomers manufactured from low molecular weight polymerisation of tetrafluoroethylene. 
Examples

Fluorotelomer alcohol (FTOH)

A general term for substances with the general structure

$\mathrm{F}\left(\mathrm{CF}_{2} \mathrm{CF}_{2}\right)_{n} \mathrm{CH}_{2} \mathrm{CH}_{2} \mathrm{OH}$

Fluorotelomer olefin (FTO)

A general term for substances with the general structure $\mathrm{F}\left(\mathrm{CF}_{2} \mathrm{CF}_{2}\right)_{\mathrm{n}} \mathrm{CH}=\mathrm{CH}_{2}$. 


\section{Appendix B - Illustration of mapping of SPIN- and preregistered chemicals}

\section{Aggregated PFC information of SPIN- and preregistered chemicals}

There were 118 CAS numbers on the SPIN list of which 27 were polymers or not-precise defined mixtures, which are excluded from the schemes but listed in the end. 91 CAS numbers were included in the sorting. The SPIN chemicals are indicated with an asterisk (*).

There were 518 CAS numbers on the preregistration list. Of these 79 were polymers or not-precise defined mixtures which are listed at the end.

Additionally synonyms, acronyms, trade names, physical-chemical data and use data have been collected, but only included a few of these data in the tables. This can be further developed.

The applied names are as simple as possible and we have chosen to use the the ones that are easiest to understand. Those are not necessarily the most correct ones but we have made this choice to make it easier to get an overview and see homologue rows and relationships. That's also why "perfluor" and fluorotelomer names have been used where possible. Fluorotelomers with a branched fluoroalkyl chain, however, have got more systematic names.

\begin{tabular}{lll} 
Perfluoroalkyl sulfonic acids (PFSAs) & \\
\hline CAS 375-73-5 & Perfluorobutane sulfonic acid, & PFBS \\
CAS 355-46-4 & Perfluorohexanesulfonic acid, & PFHxS \\
CAS 375-92-8 & Perfluoroheptane sulfonic acid, & PFHpS \\
CAS 335-77-3 & Perfluorodecane sulfonic acid, & PFDS \\
CAS 79780-39-5 & Perfluorododecane sulfonic acid & PFDoS \\
CAS 70259-86-8 & 4H-Perfluorobutane sulfonic acid & \\
\hline
\end{tabular}


Perfluoroalkyl sulfonates (salts)

\begin{tabular}{lll}
\hline *CAS 29420-49-3 & Potassium perfluorobutane -sulfonate, & PFBS-K \\
*CAS 3872-25-1 & Potassium perfluoropentane sulfonate, & PFPS-K \\
*CAS 3871-99-6 & Potassium perfluorohexane -sulfonate, & PFHxS-K \\
${ }^{*}$ CAS 60270-55-5 & Potassium perfluoroheptane-sulfonate, & PFHpS-K \\
CAS 85187-17-3 & Potassium perfluorododecane sulfonate, & PFDS \\
CAS 70259-85-7 & Potassium 4H-perfluorobutane sulfonate & \\
CAS 68259-10-9 & Ammonium perfluoro-butanesulfonate & \\
CAS 68259-09-6 & Ammonium perfluoro-pentane sulfonate & \\
CAS 68259-08-5 & Ammonium perfluoro-hexane sulfonate & \\
CAS 68259-07-4 & Ammonium perfluoroheptane sulfonate & \\
*CAS 17202-41-4 & Ammonium perfluorononane sulfonate & \\
${ }^{*}$ CAS 67906-42-7 & Ammonium perfluorodecanesulfonate & \\
*CAS 54950-05-9 & Sodium 1,4-dioxo-1,4-bis(3,3,4,4,5,5,6,6,7,7,8,8,8- & \\
& tridecafluorooctoxy)butane-2-sulfonate & \\
*CAS 70225-15-9 & Bis(2-hydroxyethyl)ammonium perfluoroheptane sulfonate & \\
*CAS 70225-16-0 & Bis(2-hydroxyethyl)ammonium perfluorohexane sulfonate & \\
*CAS 70225-17-1 & Bis(2-hydroxyethyl) ammonium perfluoropentane sulfonate & \\
*CAS 70225-18-2 & Bis(2-hydroxyethyl) ammonium perfluorobutane sulfonate & \\
CAS 56773-42-3 & Tetraethyl ammonium heptadecafluorooctane sulfonate & Metal plating, Fumetrol 108, \\
CAS 220689-12-3 & Tetrabutyl phosphonium perfluorobutane sulfonate & Fluortensid FT 248, \\
\hline
\end{tabular}

\section{Perfluoroalkyl sulfinic acid/sulfinates}

CAS 68555-66-8 Sodium perfluoroheptane -sulfinate

CAS 68555-67-9 sodium perfluorooctane sulfinate

C8 Chemical

\section{Perfluorocycloalkyl sulfonic acid and derivatives}

$\begin{array}{ll}\text { CAS 335-24-0 } & \text { Potassium perfluoro-4-ethyl cyclohexane sulfonate } \\ \text { *CAS 68156-01-4 } & \text { Potassium perfluoro[1,2-dimethylcyclohexane] sulfonate } \\ \text { *CAS 68156-07-0 } & \text { Potassium perfluoro[1-methylcyclohexane] sulfonate } \\ \text { CAS 355-03-3 } & \text { Perfluorocyclohexane sulfonyl fluoride } \\ \text { CAS 68318-34-3 } & \text { Perfluoro(2-methylcyclohexane) sulfonyl fluoride } \\ \text { CAS 68156-06-9 } & \text { Perfluoro[4-methylcyclohexane] sulfonyl fluoride } \\ \text { CAS 68156-00-3 } & \text { Perfluoro[1,2-dimethylcyclohexane] sulfonyl fluoride }\end{array}$

\section{Perfluoroalkyl sulfonamides (FASAs)}

\begin{tabular}{lll}
\hline CAS 68298-12-4 & N-Methyl perfluorobutane sulfonamide & FBSA \\
CAS 68298-13-5 & N-Methyl perfluoropentane sulfonamide & \\
CAS 68259-15-4 & N-Methyl perfluorohexane sulfonamide & \\
CAS 68259-14-3 & N-Methyl perfluoroheptane sulfonamide & \\
*CAS 68957-62-0 & N-Ethyl perfluoroheptane sulfonamide & \\
CAS 68298-10-2 & N-(Phenylmethyl) perfluoroheptane sulfonamide & \\
*CAS 34449-89-3 & N-Ethyl-N-(2-hydroxyethyl)perfluorobutane sulfonamide & \\
*CAS 34454-97-2 & N-(2-hydroxyethyl)-N-methyl perfluorobutane sulfonamide/ & MeFBSE \\
CAS 40630-65-7 & N-Methyl perfluorobutane sulfonamidoethanol & AlFBSE \\
CAS 335-97-7 & N-Allyl perfluorobutane sulfonamide & \\
CAS 67584-48-9 & N-Allyl perfluorohexane sulfonamide & \\
CAS 67584-49-0 & N-Allyl perfluoroheptane sulfonamide \\
CAS 67906-41-6 & N-Allyl -N-ethyl perfluoroheptane sulfonamide \\
CAS 93894-53-2 & N-(2-Hydroxyethyl)-N-methyl 4H-perfluorobutane sulfonamide & \\
*CAS 68555-74-8 & N-(2-Hydroxyethyl)-N-methyl perfluoropentane sulfonamide & \\
\hline
\end{tabular}




\begin{tabular}{ll}
\hline${ }^{*}$ CAS 68555-75-9 & N-(2-Hydroxyethyl)-N-methyl perfluorohexane sulfonamide \\
${ }^{*}$ CAS 68555-76-0 & N-(2-Hydroxyethyl)-N-methyl perfluoroheptane sulfonamide \\
${ }^{*}$ CAS 68555-72-6 & N-Ethyl-N-(2-hydroxyethyl) perfluoropentane sulfonamide \\
${ }^{*}$ CAS 34455-03-3 & N-Ethyl-N-(2-hydroxyethyl) perfluorohexane sulfonamide \\
${ }^{*}$ CAS 68555-73-7 & N-Ethyl-N-(2-hydroxyethyl) perfluoroheptane sulfonamide \\
CAS 85665-64-1 & N-(2-Hydroxyethyl)-N-propyl perfluorohexane sulfonamide \\
CAS 68310-02-1 & N-Butyl-N-(2-hydroxyethyl) perfluoroheptane sulfonamide \\
CAS 93894-54-3 & N,N-Bis(2-hydroxyethyl) 4H-perfluorobutane sulfonamide \\
CAS 34455-00-0 & N,N-Bis(2-hydroxyethyl) perfluorobutane sulfonamide \\
CAS 812-94-2 & N-(4-Hydroxybutyl)-N-methyl perfluorobutane sulfonamide \\
CAS 68239-72-5 & $\mathrm{N}$-(4-Hydroxybutyl)-N-methyl perfluoropentane sulfonamide, \\
CAS 68239-74-7 & $\mathrm{N}$-(4-Hydroxybutyl)-N-methyl perfluorohexane sulfonamide \\
CAS 68298-89-5 & $\mathrm{N}$-(4-Hydroxybutyl)-N-Methyl perfluoroheptane sulfonamide \\
CAS 68555-77-1 & $\mathrm{N}$-[3-(Dimethylamino)propyl] perfluorobutane sulfonamide \\
CAS 68555-78-2 & $\mathrm{N}$-[3-(Dimethylamino)propyl] perfluoropentane sulfonamide \\
CAS 50598-28-2 & $\mathrm{N}$-[3-(Dimethylamino)propyl] perfluorohexane sulfonamide \\
CAS 67584-54-7 & $\mathrm{N}$-[3-(Dimethylamino)propyl] perfluoroheptane sulfonamide \\
CAS 67584-63-8 & Perfluorobutane sulfonamide, N-ethyl-N-ethyl (ethyl acetate)
\end{tabular}

\section{Perfluoroalkyl sulfonamide, quaternary ammonium salts}

CAS 68957-59-5 Perfluorobutane sulfonamide, N-[3-(dimethylamino)propyl)]-, hydrochloride

CAS 68957-60-8 Perfluoropentane sulfonamide, N-[3-(dimethylamino)propyl)]-,-hydrochloride

CAS 68957-61-9 Perfluorohexane sulfonamide, N-[3-(dimethylamino)propyl)]-,-hydrochloride

CAS 67940-02-7 Perfluoroheptane sulfonamide, N-[3-(dimethylamino)propyl)]-,-hydrochloride

CAS 38850-52-1 Perfluorohexane sulfonamide, $N$-carboxymethyl- $N-\left(N^{\prime} N^{\prime} N^{\prime}\right.$-trimethylpropanaminium

*CAS 38850-58-7 Perfluorohexane sulfonamide, $\mathrm{N}$-sulfoxypropyl-N-( $\mathrm{N}^{\prime}, \mathrm{N}^{\prime}$-dimethyl- $\mathrm{N}^{\prime}$-hydroxyethyl propanaminium)

CAS 38850-60-1 Perfluorohexane sulfonamide, $\mathrm{N}$-sulfoxypropyl-N-( $\mathrm{N}^{\prime}, \mathrm{N}^{\prime}$-dimethyl-propanaminium)

*CAS 52166-82-2 Perfluorohexane sulfonamide, $N$ - $\left(\mathrm{N}^{\prime}, \mathrm{N}^{\prime}, \mathrm{N}^{\prime}\right.$-trimethyl propanaminium) chloride

*CAS 53518-00-6 Perfluorobutane sulfonamide, $N$ - $\left(N^{\prime}, N^{\prime}, N^{\prime}\right.$-trimethyl propanaminium) chloride

CAS 67939-95-1 Perfluorobutane sulfonamide, $N$ - $\left(\mathrm{N}^{\prime}, \mathrm{N}^{\prime}, \mathrm{N}^{\prime}\right.$-trimethyl propanaminium) iodide

*CAS 68957-55-1 Perfluoropentane sulfonamide $N$ - $\left(\mathrm{N}^{\prime}, \mathrm{N}^{\prime}, \mathrm{N}^{\prime}\right.$-trimethyl propanaminium) chloride

*CAS 68957-57-3 Perfluoropentane sulfonamide $N$ - $\left(N^{\prime}, N^{\prime}, N^{\prime}\right.$-trimethyl propanaminium) iodide

*CAS 68957-58-4 Perfluorohexane sulfonamide $\mathrm{N}-\left(\mathrm{N}^{\prime}, \mathrm{N}^{\prime}, \mathrm{N}^{\prime}\right.$-trimethyl propanaminium) iodide

*CAS 68555-81-7 Perfluoroheptane sulfonamide $\mathrm{N}-\left(\mathrm{N}^{\prime}, \mathrm{N}^{\prime}, \mathrm{N}^{\prime}\right.$-trimethyl propanaminium) chloride

*CAS 67584-58-1 Perfluoroheptane sulfonamide $N$ - $\left(N^{\prime}, N^{\prime}, N^{\prime}\right.$-trimethyl propanaminium) iodide

CAS 70225-22-8 Di[Perfluorobutane sulfonamide $\mathrm{N}-\left(\mathrm{N}^{\prime}, \mathrm{N}^{\prime}, \mathrm{N}^{\prime}\right.$-trimethyl propanaminium)] sulfate

CAS 70225-24-0 Di[Perfluoropentane sulfonamide $N-\left(N^{\prime}, N^{\prime}, N^{\prime}\right.$-trimethyl propanaminium)] sulfate

CAS 70248-52-1 Di[Perfluorohexane sulfonamide $N-\left(N^{\prime}, N^{\prime}, N^{\prime}\right.$-trimethyl propanaminium)] sulfate

CAS 70225-20-6 Di[Perfluoroheptane sulfonamide $N-\left(N^{\prime}, N^{\prime}, N^{\prime}\right.$-trimethyl propanaminium) $]$ sulfate 
Perfluoroalkyl sulfonamide acrylates (MeFASACs)

*CAS 67584-55-8 Perfluorobutane sulfonamide, N-methyl -N-ethyl acrylate/

N-Methyl perfluorobutane sulfonamidoethyl acrylate

*CAS 67584-56-9 Perfluoropentane sulfonamide, $\mathrm{N}$-methyl -N-ethyl acrylate

*CAS 67584-57-0 Perfluorohexane sulfonamide, N-methyl -N-ethyl acrylate

*CAS 68084-62-8 Perfluoroheptane sulfonamide, $\mathrm{N}$-methyl-N-ethyl acrylate

CAS 17329-79-2 Perfluorobutane sulfonamide, N-ethyl-N-ethyl acrylate

CAS 68298-06-6 Perfluoropentane sulfonamide $\mathrm{N}$-ethyl-N-ethyl acrylate

CAS 1893-52-3 Perfluorohexane sulfonamide, N-ethyl-N-ethyl acrylate

CAS 59071-10-2 Perfluoroheptane sulfonamide, N-ethyl-N-ethyl acrylate

CAS 1492-87-1 Perfluorobutane sulfonamide, $\mathrm{N}$-methyl- $\mathrm{N}$-butyl acrylate

CAS 68227-99-6 Perfluoropentane sulfonamide, $\mathrm{N}$-methyl-N-butyl acrylate

CAS 68227-98-5 Perfluorohexane sulfonamide, N-methyl-N-butyl acrylate

*CAS 68298-60-2 Perfluoroheptane sulfonamide, $\mathrm{N}$-butyl-N-ethyl acrylate

CAS 66008-70-6 1H,1H-Perfluoroheptane sulfonamide, $\mathrm{N}$-methyl-N-ethyl acrylate

*CAS 49859-70-3 1 $1 \mathrm{H}, 1 \mathrm{H}$-Perfluorooctane sulfonamide, $\mathrm{N}$-methyl-N-ethyl acrylate

CAS 66008-69-3 $\quad 1 \mathrm{H}, 1 \mathrm{H}$-Perfluorononane sulfonamide, $\mathrm{N}$-methyl- $\mathrm{N}$-ethyl acrylate

CAS 66008-68-2 $\quad 1 \mathrm{H}, 1 \mathrm{H}$-Perfluoroundecane sulfonamide, $\mathrm{N}$-methyl-N-ethyl acrylate

*CAS 72276-05-2 $1 \mathrm{H}, 1 \mathrm{H}$-Perfluorododecane sulfonamide, $\mathrm{N}$-methyl-N-ethyl acrylate

CAS 66008-67-1 $\quad 1 \mathrm{H}, 1 \mathrm{H}$-Perfluorotridecane sulfonamide, $\mathrm{N}$-methyl- $\mathrm{N}$-ethyl acrylate

C8 Chemical

CAS 72276-06-3 $\quad 1 \mathrm{H}, 1 \mathrm{H}$-Perfluorotetradecane sulfonamide, $\mathrm{N}$-methyl- $\mathrm{N}$-ethyl acrylate

CAS 68758-55-4 $\quad 1 \mathrm{H}, 1 \mathrm{H}$-Perfluoro-pentadecyl sulfonamide $\mathrm{N}$-methyl-N-ethyl acrylate

CAS 68758-56-5 1H,1 $\quad 1 \mathrm{H}$-Perfluoroheptadecyl sulfonamide $\mathrm{N}$-methyl-N-ethyl acrylate

\section{Perfluoroalkyl sulfonamide methacrylates}

*CAS 67584-59-2 Perfluorobutane sulfonamide, N-methyl-N-ethyl methacrylate

*CAS 67584-60-5 Perfluoropentane sulfonamide, N-methyl-N-ethyl methacrylate

*CAS 67584-61-6 Perfluorohexane sulfonamide, N-methyl-N-ethyl methacrylate

CAS 67939-96-2 Perfluoroheptane sulfonamide, N-methyl-N-ethyl methacrylate

CAS 67939-33-7 Perfluorobutane sulfonamide, N-ethyl-N-ethyl methacrylate

CAS 67906-73-4 Perfluoropentane sulfonamide, $\mathrm{N}$-ethyl-N-ethyl methacrylate

CAS 67906-70-1 Perfluorohexane sulfonamide, N-ethyl-N-ethyl methacrylate

CAS 67939-36-0 Perfluoroheptane sulfonamide, N-ethyl-N-ethyl methacrylate

CAS 67906-39-2 Perfluorobutane sulfonamide, $\mathrm{N}$-methyl-N-butyl methacrylate

CAS 67906-40-5 Perfluoropentane sulfonamide, N-methyl-N-butyl methacrylate

CAS 67939-61-1 Perfluorohexane sulfonamide, N-methyl-N-butyl methacrylate

CAS 68227-97-4 Perfluoroheptane sulfonamide, N-methyl-N-butyl methacrylate

\section{Perfluoroalkyl sulfonamide phosphates}

CAS 67939-89-3 [Perfluorobutane sulfonamide-N-ethyl]-N-ethyl dihydrogen-phosphate

CAS 67939-90-6 [Perfluoropentane sulfonamide-N-ethyl]-N-ethyl dihydrogen-phosphate

CAS 67969-65-7 [Perfluoroheptane sulfonamide-N-ethyl]-N-ethyl dihydrogen-phosphate

$\begin{array}{lll}\text { CAS 67969-65-7 } & \text { [Perfluoroheptane sulfonamide-N-ethyl]-N-ethyl dihydrogen-phosphate } & \text { MonoPAP } \\ \text { CAS 67923-61-9 } & \text { [Perfluoroheptane sulfonamide-N-ethyl]-N,N'-diethyl dihydrogen-phosphate } & \text { DiPAP }\end{array}$

MonoPAP

CAS 67939-98-4 Diammonium [Perfluoroheptane sulfonamide-N-ethyl]-N, $\mathrm{N}^{\prime}$-diethyl

DiPAP

dihydrogenphosphate

CAS 67939-91-7 Di[perfluorobutane sulfonamide $\mathrm{N}$-ethyl]-N, $\mathrm{N}^{\prime}$-diethyl phosphate

CAS $67939-87-1$

Di[perfluoropentane sulfonamide $\mathrm{N}$-ethyl]- $\mathrm{N}, \mathrm{N}^{\prime}$-diethyl phosphate

CAS 67939-92-8

Di[perfluorohexane sulfonamide $\mathrm{N}$-ethyl]-N, $\mathrm{N}^{\prime}$-diethyl phosphate

DiPAP

Di[perfluoroheptane sulfonamide $\mathrm{N}$-ethyl]- $\mathrm{N}, \mathrm{N}^{\prime}$-diethyl phosphate

DiPAP

CAS 67939-93-9

Di[perfluoroheptane sulfonamide $\mathrm{N}$-ethyl]- $\mathrm{N}, \mathrm{N}^{\prime}$-diethyl phosphate
Ammonium di[perfluoroheptane sulfonamide $\mathrm{N}$-ethyl]- $\mathrm{N}, \mathrm{N}^{\prime}$-diethyl phosphate

CAS 67939-97-3

DIPAP

CAS 67939-94-0

Tri[perfluoroheptane sulfonamide $\mathrm{N}$-ethyl] $-\mathrm{N}, \mathrm{N}^{\prime}, \mathrm{N}^{\prime \prime}$-triethyl phosphate

DiPAP

DiPAP

TriPAP 
Perfluoroalkyl sulfonyl halides

$\begin{array}{ll}\text { CAS 375-72-4 } & \text { Perfluorobutane sulfonyl fluoride } \\ \text { CAS 90268-45-4 } & \text { Perfluorobutane sulfonyl fluoride, branched } \\ \text { CAS 375-81-5 } & \text { Perfluoropentane sulfonyl fluoride } \\ \text { CAS 335-71-7 } & \text { Perfluoroheptane sulfonyl fluoride } \\ \text { CAS 55591-23-6 } & \text { Perfluorohexane sulfonyl chloride } \\ \text { CAS 68259-06-3 } & \text { Perfluorononane sulfonyl fluoride } \\ \text { CAS 51947-19-4 } & \text { 4-Perfluoroalkenoxybenzene -sulfonyl chloride }\end{array}$

\section{Other polyfluoroalkyl sulfur compounds}

\begin{tabular}{|c|c|c|}
\hline CAS 36913-91-4 & Perfluorobutane sulfonic -anhydride & \\
\hline CAS 93894-55-4 & $4 \mathrm{H}$-Perfluorobutane sulfonic -anhydride & \\
\hline CAS 68957-33-5 & N-Ethyl-N-perfluorobutyl sulfonyl glycine & \\
\hline CAS 68957-31-3 & N-Ethyl-N-perfluoropentyl-sulfonyl glycine & \\
\hline CAS 68957-32-4 & N-Ethyl-N-perfluorohexyl sulfonyl glycine & \\
\hline CAS 68957-63-1 & N-Ethyl-N-perfluoroheptyl-sulfonyl glycine & \\
\hline CAS 68555-79-3 & Ethyl N-ethyl-N-perfluoropentyl sulfonyl glycinate & \\
\hline CAS 68957-53-9 & Ethyl N-ethyl-N-perfluorohexyl sulfonyl glycinate & \\
\hline CAS 68957-54-0 & Ethyl N-ethyl-N-perfluoroheptyl sulfonyl glycinate & \\
\hline *CAS 67584-51-4 & Potassium N-ethyl-N-perfluorobutyl sulfonyl glycinate & \\
\hline *CAS 67584-52-5 & Potassium N-ethyl-N-perfluoropentyl sulfonyl glycinate & \\
\hline *CAS 67584-53-6 & Potassium N-ethyl-N-perfluorohexyl sulfonyl glycinate & \\
\hline *CAS 67584-62-7 & Potassium N-ethyl-N-perfluoroheptyl sulfonyl glycinate & \\
\hline *CAS 68900-97-0 & Chromium(III) N-ethyl-N-perfluorobutyl sulfonyl glycinate & \\
\hline *CAS 68891-99-6 & Chromium(III) N-ethyl-N-perfluoropentyl sulfonyl glycinate & \\
\hline *CAS 68891-98-5 & Chromium(III) N-ethyl-N-perfluorohexyl sulfonyl glycinate & \\
\hline *CAS 68891-97-4 & Chromium(III) N-ethyl-N-perfluoroheptyl sulfonyl glycinate & \\
\hline CAS $68555-68-0$ & Sodium N-ethyl-N-perfluorobutyl sulfonyl glycinate & \\
\hline CAS 68555-69-1 & Sodium N-ethyl-N-perfluoropentyl sulfonyl glycinate & \\
\hline CAS $68555-70-4$ & Sodium N-ethyl-N-perfluorohexyl sulfonyl glycinate & \\
\hline CAS $68555-71-5$ & Sodium N-ethyl-N-perfluoroheptyl sulfonyl glycinate & \\
\hline CAS 52584-45-9 & 4-Perfluoroalkenoxybenzene sulfonic acid & \\
\hline CAS 68299-19-4 & Sodium (perfluorobutylsulfonyl)aminomethyl benzene sulfonate & \\
\hline CAS 68299-20-7 & Sodium (perfluoropentylsulfonyl)aminomethyl benzene sulfonate & \\
\hline CAS $68299-21-8$ & Sodium (perfluorohexylsulfonyl)-aminomethyl benzene sulfonate & \\
\hline CAS 68299-29-6 & Sodium (perfluoroheptylsulfonyl)-aminomethyl benzene sulfonate & \\
\hline *CAS 68649-26-3 & Reaction product with PFOS and PFBS derivatives & C8 chemical \\
\hline *CAS 68541-01-5 & Perfluoroheptane sulfonic acid ester with complex alcohol & $\begin{array}{l}\text { Tetrachloro-phthalic } \\
\text { acid derivative }\end{array}$ \\
\hline *CAS 68541-02-6 & Perfluoropentane sulfonic acid ester with complex alcohol & $\begin{array}{l}\text { Tetrachloro-phthalic } \\
\text { acid derivative }\end{array}$ \\
\hline *CAS 68568-54-7 & Perfluorobutane sulfonic acid ester with complex alcohol & $\begin{array}{l}\text { Tetrachloro-phthalic } \\
\text { acid derivative }\end{array}$ \\
\hline CAS 69013-34-9 & $\begin{array}{l}\text { N-Methyl-4-[[4,4,5,5,5-pentafluoro-3-(1,1,2,2,2-pentafluoroethyl)- } \\
\text { 1,2,3-tris(trifluoromethyl)-1-penten-1-yl]oxy]-N-[2- } \\
\text { (phosphonooxy)ethyl]-benzene sulfonamide }\end{array}$ & \\
\hline
\end{tabular}


Perfluoroalkyl carboxylic acids (PFCA)

\begin{tabular}{lll}
\hline *CAS 2706-90-3 & Perfluoropentanoic acid & PFPA \\
*CAS 307-24-4 & Perfluorohexanoic acid & PFHxA \\
*CAS 375-85-9 & Perfluoroheptanoic acid & PFHpA \\
CAS 375-95-1 & Perfluorononanoic acid & PFNA \\
CAS 15899-31-7 & Perfluoroisononanoic acid & \\
CAS 335-76-2 & Perfluorodecanoic acid & PFDA, C10 \\
CAS 2058-94-8 & Perfluoroundecanoic acid & PFuDA, C11 \\
CAS 16486-94-5 & Perfluoroisoundecanoic acid & \\
CAS 307-55-1 & Perfluorododecanoic acid & PFDoA, C12 \\
CAS 72629-94-8 & Perfluorotridecanoic acid & C13 \\
CAS 16486-96-7 & Perfluoroisotridecanoic acid & \\
CAS 376-06-7 & Perfluorotetradecanoic acid & C14 \\
CAS 18024-09-4 & Perfluoropentadecanoic acid & C15 \\
CAS 18024-09-4 & Perfluoroisopentadecanoic acid & \\
CAS 67905-19-5 & Perfluorohexadecanoic acid & C16 \\
CAS 16517-11-6 & Perfluorostearic acid & C18 \\
CAS 68310-12-3 & Perfluoroeicosanoic acid & C20 \\
CAS 336-08-3 & Perfluoroadipic acid & \\
CAS 376-72-7 & 5H-Perfluoropentanoic acid & \\
CAS 1546-95-8 & 7H-Perfluoroheptanoic acid & \\
CAS 76-21-1 & 9H-perfluorononanoic acid & 11H-Perfluoroundecanoic acid \\
CAS 1765-48-6 & &
\end{tabular}

\section{Perfluoroalkyl carboxylic salts}

\begin{tabular}{lll}
\hline CAS 2706-89-0 & Sodium perfluoropentanoate & PFPA \\
CAS 20109-59-5 & Sodium perfluoroheptanoate & PFHpA \\
CAS 68259-11-0 & Ammonium perfluoropentanoate & PFPA \\
CAS 21615-47-4 & Ammonium perfluorohexanoate & PFHXA \\
CAS 6130-43-4 & Ammonium perfluoroheptanoate & PFHpA \\
CAS 3658-62-6 & Ammonium perfluoro-isononanoate & PFiNA \\
CAS 3108-42-7 & Ammonium perfluorodecanoate & PFDA \\
CAS 3658-63-7 & Ammonium perfluoro-isoundecanoate & \\
CAS 3793-74-6 & Ammonium perfluorododecanoate & \\
CAS 22715-45-3 & Ammonium 5H-perfluoropentanoate & \\
CAS 376-34-1 & Ammonium 7H-perfluoroheptanoate & \\
CAS 1868-86-6 & Ammonium 9H-perfluorononanoate & \\
CAS 307-71-1 & Potassium 11H-Perfluoroundecanoate & \\
CAS 3658-57-9 & Ammonium 7-(chlorodifluoromethyl)perfluorooctanoate \\
CAS 16557-94-1 & Ammonium 7-(chlorodifluoromethyl)perfluoroheptanoate & \\
CAS 68015-84-9 & Ethylammonium perfluoro-isohexanoate & \\
CAS 68015-86-1 & Ethylammonium perfluoro-isooctanoate & \\
CAS 68015-85-0 & Ethylammonium perfluoro-isodecanoate & \\
CAS 68015-87-2 & Ethylammonium perfluoro-isododecanoate \\
CAS 68052-68-6 & Ethylammonium perfluoro-isopentadecanoate & \\
\hline
\end{tabular}


Perfluoroalkyl carboxylic acid halides

\begin{tabular}{ll}
\hline CAS 375-62-2 & Perfluoropentanoyl fluoride \\
CAS 355-38-4 & Perfluorohexanoyl fluoride \\
CAS 375-84-8 & Perfluoroheptanoyl fluoride \\
CAS 18017-31-7 & Perfluoroisohexanoyl fluoride \\
CAS 15899-29-3 & Perfluoroisoheptanoyl fluoride \\
CAS 15742-62-8 & Perfluoroisononanoyl fluoride \\
CAS 15720-98-6 & Perfluoroisoundecanoyl fluoride \\
CAS: 15811-52-6 & Perfluoroisotridecanoyl fluoride \\
CAS 68025-62-7 & Perfluoroisopentadecanoyl fluoride \\
CAS 37881-62-2 & Perfluorohexanedioyl difluoride \\
CAS 423-95-0 & 9H-Perfluorononanoyl chloride \\
CAS 64018-26-4 & 1H,1H-Perfluorododecanoyl chloride
\end{tabular}

Perfluoroalkyl alcohols/ketones

\begin{tabular}{ll}
\hline CAS 355-80-6 & 1H,1H,5H-perfluoropentanol \\
CAS 375-82-6 & 1H,1H-Perfluoroheptanol \\
CAS 335-99-9 & 1H,1H,7H-Perfluoroheptanol \\
CAS 307-30-2 & 1H,1H-Perfluorooctanol \\
*CAS 376-18-1 & 1H,1H,9H-Perfluorononanol \\
CAS 307-70-0 & 1H,1H,11H-Perfluoroundecanol \\
CAS 67824-44-6 & 3-Perfluoroisononyl-propane-1,2-diol \\
CAS 94159-92-9 & 1-Phenoxy-3-perfluoroisononyl-2-propanol \\
CAS 94158-62-0 & 1-[2-(2-butoxyethoxy)ethoxy]-3-perfluoroisononyl-propan-2-ol \\
CAS 93776-07-9 & 32-(Perfluorodecyl)-2,5,8,11,14,17,20,23,26-decaoxatetratetracontan-31-ol \\
CAS 93776-06-8 & 32-(Perfluorododecyl)-2,5,8,11,14,17,20,23,26-decaoxatetratetracontan-31-ol \\
CAS 93776-09-1 & 32-(Perfluoroisotridecyl)-2,5,8,11,14,17,20,23,26-decaoxatetratetracontan-31-ol \\
CAS 93776-11-5 & 32-(Perfluoroisononyl)- 31-hydroxy-dotetracontane-2,5,8,11,14,17,20,23,26,29-decone \\
CAS 93776-10-4 & 32-(Perfluoroisoundecyl)-31-hydroxy-dotetracontane-2,5,8,11,14,17,20,23,26,29-decone \\
\hline
\end{tabular}

Perfluoroalkyl halides

\begin{tabular}{lll}
\hline CAS 375-88-2 & Perfluoroheptyl bromide & C7 \\
CAS 307-43-7 & Perfluorodecyl bromide & C10 \\
CAS 25398-32-7 & Perfluoroalkyl iodides & Zonyl TELA-N \\
CAS 423-39-2 & Perfluorobutyl iodide & C4 \\
CAS 638-79-9 & Perfluoropentyl iodide & C5 \\
CAS 355-43-1 & Perfluorohexyl iodide & C6 \\
CAS 335-58-0 & Perfluoroheptyl iodide & C7 \\
CAS 507-63-1 & Perfluorooctyl iodide & C8 chemical \\
CAS 558-97-4 & Perfluorononyl iodide & C9 \\
CAS 865-77-0 & Perfluoroisononyl iodide & C9 \\
CAS 423-62-1 & Perfluorodecyl iodide & C10 \\
CAS 677-93-0 & Perfluoroisoundecyl iodide & C11 \\
CAS 307-60-8 & Perfluorododecyl iodide & C12 \\
CAS 307-63-1 & Perfluorotetradecyl iodide & C14 \\
CAS 3248-61-1 & Perfluoroisotridecyl iodide & C13 \\
CAS 3248-63-3 & Perfluoroisopentadecyl iodide & C15 \\
CAS 355-50-0 & Perfluorohexadecyl iodide & C16 \\
CAS 29809-35-6 & Perfluorooctadecyl iodide & C18 \\
CAS 29809-34-5 & Perfluoroeicosyl iodide & C20 \\
CAS 29809-36-7 & Perfluorodocosanyl iodide & C22 \\
CAS 39823-55-7 & Perfluorotetracosyl iodide & C24 \\
\hline
\end{tabular}




\begin{tabular}{|c|c|c|}
\hline CAS $65975-15-7$ & Perfluorohexacosanyl iodide & \\
\hline CAS $375-50-8$ & 1,4-diiodoperfluorobutane & \\
\hline CAS $375-80-4$ & 1,6-Diiodoperfluorohexane & \\
\hline \multicolumn{3}{|c|}{ Perfluoroalkyl alkyl ethers } \\
\hline *CAS 297730-93-9 & Ethyl perfluoroisoheptyl ether & Novec Engineered Fluid HFE 7500 \\
\hline *CAS $163702-08-7$ & Methyl perfluoroisobutyl ether & $\begin{array}{l}\text { 3M Novec Engineered Fluid HFE-7100 } \\
\text { (Mixture with CAS 163702-07-6. }\end{array}$ \\
\hline *CAS $163702-07-6$ & Methyl perfluorobutyl ether & $\begin{array}{l}\text { Cosmetic Fluid CF } 61 ; 3 \mathrm{M} \text { Novec } \\
\text { Engineered Fluid HFE-7100 (Mixture } \\
\text { with CAS 163702-08-7) }\end{array}$ \\
\hline *CAS 163702-05-4 & Ethyl perfluorobutyl ether & \\
\hline CAS 66396-73-4 & $4 \mathrm{H}$-Perfluorobutyl vinyl ether & \\
\hline CAS 78971-81-0 & $1 \mathrm{H}, 1 \mathrm{H}, 7 \mathrm{H}$-Perfluoroheptyl vinyl ether & \\
\hline CAS 71726-31-3 & $1 \mathrm{H}, 1 \mathrm{H}, 9 \mathrm{H}$-Perfluorononyl vinyl ether & \\
\hline CAS 94231-58-0 & $1 \mathrm{H}, 1 \mathrm{H}, 11 \mathrm{H}$-Perfluoroundecyl vinyl ether & \\
\hline CAS $73928-40-2$ & Perfluorovinyl $5 \mathrm{H}$-perfluoropentane ether & \\
\hline CAS 70729-63-4 & $\begin{array}{l}\text { Tributyl ammonium 4-((4,4,5,5,5-pentafluoro-3-(pentafluoroethyl)- } \\
\text { 1,2,3-tris(trifluoromethyl)pent-1-enyl)oxy)benzene sulfonate }\end{array}$ & \\
\hline CAS 84029-54-9 & Tetratriacontafluoro-10,13,16,19-tetraoxaoctacosadiene & \\
\hline CAS 93776-05-7 & Bis(1-perfluoroiisononyl-4-methyl- 3-oxy-2-hexanol) ether & \\
\hline CAS 93776-01-3 & Bis(1-perfluorodecyl-4-methyl- 3-oxy-2-hexanol) ether & \\
\hline CAS 93776-04-6 & Bis(1-perfluoroisoundecyl-4-methyl-3-oxy-2-hexanol) ether & \\
\hline CAS $93776-00-2$ & Bis(1-perfluorododecyl-4-methyl- 3-oxy-2-hexanol) ether & \\
\hline CAS 93776-03-5 & Bis(1-perfluoroisotridecyl-4-methyl-3-oxy-2-hexanol) ether & \\
\hline \multicolumn{3}{|c|}{ Perfluoroalkyl amines } \\
\hline CAS 311-89-7 & Tri(perfluorobutyl)amine & \\
\hline CAS $338-84-1$ & Tri(perfluoropentyl)amine & \\
\hline CAS $31841-41-5$ & $\mathrm{~N}, \mathrm{~N}$-bis(2-hydroxyethyl)-N-methyl ammonium iodide & C8 chemical \\
\hline CAS 80909-29-1 & Perfluoroisononyl 2-ethyl-propyl trimethyl ammonium iodide & \\
\hline CAS 94159-78-1 & $\begin{array}{l}\mathrm{N}, \mathrm{N}-\mathrm{Bis}(2 \text {-hydroxyethyl)-N-methyl-N-[(2-hydroxy-3- } \\
\text { perfluoroisononyl)propyl] ammonium iodide }\end{array}$ & \\
\hline CAS 93776-17-1 & $\begin{array}{l}\mathrm{N}, \mathrm{N} \text {-Bis(2-hydroxyethyl)-N-methyl-N-[(2-hydroxy-3- } \\
\text { perfluorodecyl)propyl] ammonium iodide }\end{array}$ & \\
\hline CAS 94159-77-0 & $\begin{array}{l}\mathrm{N}, \mathrm{N} \text {-Bis(2-hydroxyethyl)-N-methyl-N-[(2-hydroxy-3- } \\
\text { perfluoroisoundecyl)propyl] ammonium iodide }\end{array}$ & \\
\hline CAS $93776-16-0$ & $\begin{array}{l}\mathrm{N}, \mathrm{N}-\mathrm{Bis}(2 \text {-hydroxyethyl)-N-methyl-N-[(2-hydroxy-3- } \\
\text { perfluorododecyl)propyl] ammonium iodide }\end{array}$ & \\
\hline CAS 94159-76-9 & $\begin{array}{l}\mathrm{N}, \mathrm{N}-\mathrm{Bis}(2 \text {-hydroxyethyl)-N-methyl-N-[(2-hydroxy-3- } \\
\text { perfluoroisotridodecyl)propyl] ammonium iodide }\end{array}$ & \\
\hline CAS 73353-26-1 & 1-[[3-(Dimethylamino)propyl]amino]-3-perfluoroisononyl-2-propanol & \\
\hline CAS $94159-80-5$ & 1-[[3-(Dimethylamino)propyl]amino]-3- perfluorodecyl-2-propanol & \\
\hline CAS 94159-83-8 & $\begin{array}{l}\text { 1-[[3-(Dimethylamino)propyl]amino]-3-perfluoroisoundecyl-2- } \\
\text { propanol }\end{array}$ & \\
\hline CAS 94159-79-2 & 1-[[3-(Dimethylamino)propyl]amino]-3-perfluoro-dodecyl-2-propanol & \\
\hline CAS 94159-82-7 & $\begin{array}{l}\text { 1-[[3-(Dimethylamino) propyl]amino]-3-perfluoro-isotridecyl-2- } \\
\text { propanol }\end{array}$ & \\
\hline
\end{tabular}


Perfluoroalkyl amino acids/salts

\begin{tabular}{ll}
\hline CAS 94159-89-4 & $\begin{array}{l}\text { Potassium N-methyl-N- [(3-perfluoro-isononyl-2- } \\
\text { hydroxy)propyl] glycinate }\end{array}$ \\
CAS 93776-13-7 & $\begin{array}{l}\text { 3-[Dimethyl-[3-[(3-perfluoro-decyl-2-hydroxy)amino]- } \\
\text { propyl]ammonio] propanoate }\end{array}$ \\
CAS 93777-12-9 & $\begin{array}{l}\text { 3-[Dimethyl-[3-[(3-perfluoro-isoun-decyl-2- } \\
\text { hydroxy)amino]-propyl]-ammonio] propanoate }\end{array}$ \\
CAS 93776-15-9 & $\begin{array}{l}\text { 3-[Dimethyl-[3-[(3-perfluoro-isotridecyl-2-hydroxy)amino]- } \\
\text { propyl]ammonio] propanoate }\end{array}$ \\
CAS 93776-12-6 & $\begin{array}{l}\text { 3-[Ethyl-[3-[(3-perfluorododecyl-2-hydroxy)amino]propyl- } \\
\text { ]ammonio]-propanoate }\end{array}$ \\
CAS 73353-25-0 & $\mathrm{N}$-[(2-Carboxyethyl)-3-[2-hydroxy-3-perfluoroisononyl]- \\
& propylamino] - N,N-dimethyl-propanaminium hydroxide
\end{tabular}

\section{Perfluoroalkyl phosphates}

\begin{tabular}{|c|c|c|}
\hline CAS $78974-42-2$ & Perfluoroisononyl ethyl dihydrogen phosphate & MonoPAP, isotelomer \\
\hline CAS $94200-56-3$ & Perfluoroisoundecyl ethyl dihydrogen phosphate & MonoPAP, isotelomer \\
\hline CAS 94200-57-4 & Perfluoroisotridecyl ethyl dihydrogen phosphate & MonoPAP, isotelomer \\
\hline CAS 93857-42-2 & Perfluoroisopentadecyl ethyl dihydrogen phosphate & MonoPAP, isotelomer \\
\hline CAS 94231-59-1 & Perfluoroisoheptadecyl ethyl dihydrogen phosphate & MonoPAP, isotelomer \\
\hline CAS 93857-49-9 & Diammonium perfluoroisononyl ethyl phosphate & MonoPAP \\
\hline CAS 93857-45-5 & Diammonium perfluorodecyl ethyl phosphate & MonoPAP \\
\hline CAS 93857-50-2 & Diammonium perfluoroisoundecyl ethyl phosphate & MonoPAP \\
\hline CAS 93857-46-6 & Diammonium perfluorododecyl ethyl phosphate & MonoPAP \\
\hline CAS 93857-51-3 & Diammonium perfluoroisotridecyl ethyl phosphate & MonoPAP \\
\hline CAS 93857-47-7 & Diammonium perfluorotetradecyl ethyl phosphate & MonoPAP \\
\hline CAS 93857-52-4 & Diammonium perfluoroisopentadecyl ethyl phosphate & MonoPAP \\
\hline CAS 93857-48-8 & Diammonium perfluorohexadecyl ethyl phosphate & MonoPAP \\
\hline CAS 93857-43-3 & Diammonium perfluoroisoheptadecyl ethyl phosphate & MonoPAP \\
\hline CAS 1895-26-7 & Di[2-(perfluorodecyl)ethyl] hydrogen phosphate & DiPAP \\
\hline CAS 78974-41-1 & Di[(2-perfluoroisononyl)ethyl] hydrogen phosphate & DiPAP \\
\hline CAS 93857-55-7 & Di[(2-perfluoroisoundecyl)ethyl] hydrogen phosphate & DiPAP \\
\hline CAS 93857-56-8 & Di[(2-perfluoroisotridecyl)ethyl] hydrogen phosphate & DiPAP \\
\hline CAS 93857-53-5 & Di[2-(perfluorotetradecyl)ethyl] hydrogen phosphate & DiPAP \\
\hline CAS $93776-29-5$ & Di[(2-perfluoroisopentadecyl)ethyl] hydrogen phosphate & DiPAP \\
\hline CAS 93857-54-6 & Di[2-(perfluorohexadecyl)ethyl] hydrogen phosphate & DiPAP \\
\hline CAS 93776-19-3 & Di[(2-perfluoroisoheptadecyl)ethyl] hydrogen phosphate & DiPAP \\
\hline CAS 93776-24-0 & Ammonium di[(2-perfluoroisononyl)ethyl] phosphate & DiPAP \\
\hline CAS 93776-21-7 & Ammonium di[(2-perfluorodecyl)ethyl] phosphate & DiPAP \\
\hline CAS 93776-25-1 & Ammonium di[(2-perfluoro-isoundecyl)ethyl] phosphate & DiPAP \\
\hline CAS 93776-22-8 & Ammonium di[(2-perfluoro-dodecyl)ethyl] phosphate & DiPAP \\
\hline CAS 93776-26-2 & Ammonium di[(2-perfluoro-isotridecyl)ethyl] phosphate & DiPAP \\
\hline CAS 93777-13-0 & Ammonium di[(2-perfluoro-tetradecyl)ethyl] phosphate & DiPAP \\
\hline CAS 93776-27-3 & Ammonium di[(2-perfluoro-isopentadecyl)ethyl] phosphate & DiPAP \\
\hline CAS 93776-23-9 & Ammonium di[(2-perfluoro-hexadecyl)ethyl] phosphate & DiPAP \\
\hline CAS 93776-28-4 & Ammonium di[(2-perfluoroisoheptadecyl)ethyl] phosphate & DiPAP \\
\hline CAS 94291-77-7 & $\begin{array}{l}\text { Bis(2-hydroxyethyl)ammonium di [(2- } \\
\text { perfluoroisononyl)ethyl] phosphate }\end{array}$ & DiPAP \\
\hline CAS 94291-78-8 & $\begin{array}{l}\text { Bis(2-hydroxyethyl)ammonium di [(2- } \\
\text { perfluoroisoundecyl)ethyl] phosphate }\end{array}$ & DiPAP \\
\hline CAS 94231-56-8 & $\begin{array}{l}\text { Bis(2-hydroxyethyl)ammonium di [(2- } \\
\text { perfluoroisotridecyl)ethyl] phosphate }\end{array}$ & DiPAP \\
\hline CAS 93776-30-8 & $\begin{array}{l}\text { Bis(2-hydroxyethyl)ammonium di [(2- } \\
\text { perfluoroisopentadecyl)ethyl] phosphate }\end{array}$ & DiPAP \\
\hline
\end{tabular}




\begin{tabular}{|c|c|c|}
\hline CAS 93776-31-9 & $\begin{array}{l}\text { Bis(2-hydroxyethyl)ammonium di [(2- } \\
\text { perfluoroisoheptadecyl)ethyl] phosphate }\end{array}$ & DiPAP \\
\hline CAS $355-86-2$ & $\operatorname{Tri}(1 \mathrm{H}, 1 \mathrm{H}, 5 \mathrm{H}$-perfluoropentyl) phosphate & TriPAP \\
\hline CAS 54009-73-3 & (2-Hydroxy-3-perfluoroisononyl))propyl dihydrogenphosphate & MonoPAP \\
\hline CAS 94158-70-0 & (2-Hydroxy-3-perfluorodecyl)-propyl dihydrogenphosphate & MonOPAP \\
\hline CAS 63295-27-2 & $\begin{array}{l}\text { (2-Hydroxy-3-perfluoroisoundecan-yl)propyl dihydrogen } \\
\text { phosphate }\end{array}$ & MonoPAP \\
\hline CAS 94200-42-7 & $\begin{array}{l}\text { (2-Hydroxy-3-perfluorododecyl)-propyl dihydrogen- } \\
\text { phosphate }\end{array}$ & MonoPAP \\
\hline CAS 63295-28-3 & $\begin{array}{l}\text { (2-Hydroxy-3-perfluoroisotridecanyl)propyl dihydrogen } \\
\text { phosphate }\end{array}$ & MonoPAP \\
\hline CAS 94200-43-8 & $\begin{array}{l}\text { (2-Hydroxy-3-perfluorotetradecyl)propyl dihydrogen- } \\
\text { phosphate }\end{array}$ & MonoPAP \\
\hline CAS 63295-29-4 & $\begin{array}{l}\text { (2-Hydroxy -3-perfluoroisopentadecanyl)propyl dihydrogen } \\
\text { phosphate }\end{array}$ & MonoPAP \\
\hline CAS 94200-44-9 & $\begin{array}{l}\text { (2-Hydroxy -3-perfluoroisohexadecanyl)propyl dihydrogen } \\
\text { phosphate }\end{array}$ & MonoPAP \\
\hline CAS 63295-18-1 & $\begin{array}{l}\text { Diammonium (2-hydroxy-3-perfluorononyl)propyl } \\
\text { phosphate }\end{array}$ & MonoPAP \\
\hline CAS 94200-46-1 & Diammonium (2-hydroxy-3-perfluorodecyl)propyl phosphate & MonoPAP \\
\hline CAS 94200-50-7 & $\begin{array}{l}\text { Diammonium (2-hydroxy-3-perfluoroisoundecyl)propyl } \\
\text { phosphate }\end{array}$ & MonoPAP \\
\hline CAS 94200-47-2 & $\begin{array}{l}\text { Diammonium (2-hydroxy-3-perfluorododecyl)propyl } \\
\text { phosphate }\end{array}$ & MonoPAP \\
\hline CAS 94200-51-8 & $\begin{array}{l}\text { Diammonium (2-hydroxy-3-per-fluoroisotridecyl)propyl } \\
\text { phosphate }\end{array}$ & MonoPAP \\
\hline CAS 94200-48-3 & $\begin{array}{l}\text { Diammonium (2-hydroxy-3-per-fluorotetradecyl)propyl } \\
\text { phosphate }\end{array}$ & MonoPAP \\
\hline CAS 94200-52-9 & $\begin{array}{l}\text { Diammonium (2-hydroxy-3-perfluoroisopentadecyl)propyl } \\
\text { phosphate }\end{array}$ & MonoPAP \\
\hline CAS 94200-49-4 & $\begin{array}{l}\text { Diammonium (2-hydroxy-3-per-fluorohexadecyl)propyl } \\
\text { phosphate }\end{array}$ & MonoPAP \\
\hline CAS 94200-53-0 & $\begin{array}{l}\text { Diammonium (2-hydroxy-3-perfluoroisoheptadecyl)propyl } \\
\text { phosphate }\end{array}$ & MonoPAP \\
\hline
\end{tabular}

Perfluoroalkyl acrylates

\begin{tabular}{ll}
\hline CAS 307-98-2 & $1 \mathrm{H}, 1 \mathrm{H}-$ Perfluorooctyl acrylate \\
CAS 4180-26-1 & $1 \mathrm{H}, 1 \mathrm{H}, 9 \mathrm{H}-$ Hexadecafluorononyl acrylate \\
\hline
\end{tabular}

Perfluoroalkyl methacrylates

*CAS 3934-23-4 1H,1H-Perfluorooctyl methacrylate

Other perfluoralkyl esters

CAS 376-50-1 Perfluoroadipic acid diethylester

\section{Perfluoroalkyl heterocyclic compounds}

\begin{tabular}{ll}
\hline CAS 38565-52-5 & 1H,1H-Perfluoroheptyl oxirane \\
CAS 38565-53-6 & $1 \mathrm{H}, 1 \mathrm{H}-$ Perfluorononyl oxirane \\
CAS 47795-34-6 & $1 \mathrm{H}, 1 \mathrm{H}$-Perfluorododecyl oxirane \\
CAS 41925-33-1 & $1 \mathrm{H}-1 \mathrm{H}-$ Perfluoroisodecyl oxirane \\
CAS 54009-78-8 & $1 \mathrm{H}, 1 \mathrm{H}$-Perfluoroisotridecanyl oxirane \\
CAS 54009-79-9 & $1 \mathrm{H}, 1 \mathrm{H}-$ Perfluoroisoheptadecanyl oxirane \\
CAS 54009-77-7 & 2H-Perfluoroisohexadecyl oxirane \\
CAS 356-47-8 & Perfluoro-2-methyl tetrahydropyran) \\
\hline
\end{tabular}




\begin{tabular}{ll}
\hline CAS 40464-54-8 & Perfluoro-2-butyl tetrahydrofuran \\
*CAS 335-36-4 & Perfluoro-2-isobutyl tetrahydrofuran \\
CAS 69661-30-9 & Perfluoro-[2,3,4,5-tetramethyl-3-ethyl] tetrahydrofuran \\
CAS 94159-90-7 & 2,2-Dimethyl-4-(1H,1H-perfluoroisodecyl)-1,3-dioxolane \\
CAS 359-71-7 & Perfluoro-N-methyl piperidine \\
CAS 564-11-4 & Perfluoro-N-ethyl piperidine \\
CAS 42060-64-0 & Perfluorosulfolane \\
CAS 71356-38-2 & $\begin{array}{l}\text { 1-(Carboxylatomethyl)-1-(2-hydroxyethyl)-4-(perfluoro-1- } \\
\text { oxodecyl) piperazinium }\end{array}$ \\
\hline
\end{tabular}

Perfluoroalkylsilanes

CAS 375-63-3 Trichloro(1,1,2,2,3,3,4,4-octafluoro-butyl)silane

CAS 67584-50-3 N-Ethyl-N-(3-(trichlorosilyl)-propyl)perfluoroheptane sulfonamide

CAS 68239-75-8 N-Ethyl-N-(3-(trimethoxysilyl) propyl) perfluoroheptane sulfonamide

\section{Fluorotelomer alcohols}

\begin{tabular}{lll}
\hline${ }^{*}$ CAS 2043-47-2 & 4:2 Fluorotelomer alcohol & $4: 2 \mathrm{FTOH}$ \\
${ }^{*}$ CAS 647-42-7 & 6:2 Fluorotelomer alcohol & $6: 2 \mathrm{FTOH}$ \\
${ }^{*}$ CAS 678-39-7 & $8: 2$ Fluorotelomer alcohol & $8: 2 \mathrm{FTOH}, \mathrm{C}$ chemical \\
${ }^{*}$ CAS 865-86-1 & $10: 2$ Fluorotelomer alcohol & $10: 2 \mathrm{FTOH}$ \\
${ }^{*}$ CAS 39239-77-5 & $12: 2$ Fluorotelomer alcohol & $12: 2 \mathrm{FTOH}$ \\
${ }^{*}$ CAS 60699-51-6 & $14: 2$ Fluorotelomer alcohol & $14: 2 \mathrm{FTOH}$ \\
*CAS 65104-67-8 & $16: 2$ Fluorotelomer alcohol & \\
CAS 65104-65-6 & $18: 2$ Fluorotelomer alcohol & \\
\hline
\end{tabular}

\section{Fluorotelomer halogenides}

\begin{tabular}{lll}
\hline CAS 2043-55-2 & 4:2 Fluorotelomer iodide & \\
CAS 1682-31-1 & 5:2 Fluorotelomer iodide & \\
CAS 2043-57-4 & 6:2 Fluorotelomer iodide & Zonyl ${ }^{\oplus}$, TELB-LN \\
CAS 2043-52-9 & 7:2 Fluorotelomer iodide & \\
${ }^{*}$ CAS 2043-53-0 & 8:2 Fluorotelomer iodide & C8 chemical, Zonyl ${ }^{\oplus}$ TELB-LN \\
CAS 65510-56-7 & 9:2 Fluorotelomer iodide & \\
CAS 2043-54-1 & 10:2 Fluorotelomer iodide & Zonyl ${ }^{\oplus, ~ T E L B-L N ~}$ \\
CAS 30046-31-2 & 12:2 Fluorotelomer iodide & \\
CAS 65104-63-4 & 18:2 Fluorotelomer iodide & \\
CAS 65510-55-6 & 14:2 Fluorotelomer iodide & \\
CAS 26650-09-9 & 6:2 Fluorotelomer thiocyanate & \\
\hline
\end{tabular}

Fluortelomer sulfonates, sulfonyl chlorides and sulfonamides

\begin{tabular}{|c|c|c|}
\hline *CAS 27619-97-2 & 6:2 Fluorotelomer sulfonic acid & $\begin{array}{l}\text { Fumetrol }{ }^{\circledR 21} \text { (metal plating), } \\
\text { Forafac } 1033\end{array}$ \\
\hline CAS 59587-38-1 & Potassium 6:2 fluortelomer sulfonate & Zonyl 1176, wetting agent \\
\hline CAS $65702-23-0$ & 5:2 Fluorotelomer sulfonyl chloride & \\
\hline CAS 65702-24-1 & 9:2 Fluorotelomer sulfonyl chloride & \\
\hline *CAS 72276-08-5 & 10:2 Fluorotelomer sulfonyl chloride & \\
\hline CAS 68758-57-6 & 12:2 Fluorotelomer sulfonyl chloride & \\
\hline *CAS 34455-29-3 & 6:2 Fluorotelomer sulfonamide $\mathrm{N}$-propylmethyl betaine & \\
\hline CAS 61798-69-4 & 6:2 Fluorotelomer sulfonamide, $\mathrm{N}$-propylethyl betaine & \\
\hline CAS $66008-71-7$ & 6:2 Fluorotelomer sulfonamide, $\mathrm{N}$-methyl- $\mathrm{N}$-propyl betaine & \\
\hline CAS $66008-72-8$ & $\begin{array}{l}\text { 6:2 Fluorotelomer sulfonamide, } N \text {-methyl- } N \text {-propan- } \\
\text { aminium } N^{\prime}-2 \text {-carboxyethyl }\end{array}$ & \\
\hline CAS 72276-07-4 & 14:2 Fluorotelomer sulfonamide, $\mathrm{N}$-methyl- $\mathrm{N}$-ethyl acrylate & \\
\hline
\end{tabular}




\begin{tabular}{lll}
\multicolumn{2}{l}{ Fluorotelomer acrylates } & \\
\hline CAS 1799-84-4 & 4:2 Fluorotelomer acrylate & \\
${ }^{*}$ CAS 17527-29-6 & 6:2 Fluorotelomer acrylate & Zonyl ${ }^{\oplus}$ TA-N $<5 \%$ \\
${ }^{*}$ CAS 27905-45-9 & 8:2 Fluorotelomer acrylate & C8 Chemical, Zonyl ${ }^{\circledR}$ TA-N $<65 \%$ \\
${ }^{*}$ CAS 17741-60-5 & 10:2 Fluorotelomer acrylate & Zonyl ${ }^{\circledR}$ TA-N $<29 \%$ \\
${ }^{*}$ CAS 34395-24-9 & 12:2 Fluorotelomer acrylate & \\
${ }^{*}$ CAS 34362-49-7 & 14:2 Fluorotelomer acrylate & \\
${ }^{*}$ CAS 65150-93-8 & 16:2 Fluorotelomer acrylate & \\
${ }^{*}$ CAS 65104-64-5 & 18:2 Fluorotelomer acrylate & \\
CAS 15577-26-1 & 2-(Perfluoroisononyl) ethyl acrylate \\
CAS 52956-81-7 & 2-(Perfluoroisoundecyl) ethyl acrylate & \\
CAS 52956-82-8 & 2-(Perfluoroisotridecyl) ethyl acrylate & \\
CAS 91615-22-4 & 2-(Perfluoroisopentadecyl) ethyl acrylate & \\
CAS 94158-63-1 & 2-(Perfluoroisoheptadecyl) ethyl acrylate & \\
\hline
\end{tabular}

\section{Fluorotelomer methacrylates}

$\begin{array}{lll}{ }^{*} \text { CAS 2144-53-8 } & \text { 6:2 Fluorotelomer methacrylate } & \text { Capstone }{ }^{\text {TM }} \text { 62-MA } \\ { }^{*} \text { CAS 1996-88-9 } & \text { 8:2 Fluorotelomer methacrylate } & \text { C8 chemical } \\ \text { CAS 2144-54-9 } & \text { 10:2 Fluorotelomer methacrylate } & \\ { }^{*} \text { CAS 6014-75-1 } & \text { 12:2 Fluorotelomer methacrylate } & \\ \text { CAS 4980-53-4 } & \text { 14:2 Fluorotelomer methacrylate } \\ \text { CAS 59778-97-1 } & \text { 16:2 Fluorotelomer methacrylate } \\ \text { CAS 65104-66-7 } & \text { 18:2 Fluorotelomer methacrylate } \\ \text { CAS 15166-00-4 } & \text { 2-(Perfluoroisononyl) ethyl methacrylate } \\ \text { CAS 74256-14-7 } & \text { 2-(Perfluoroisoundecyl) ethyl methacrylate } \\ \text { CAS 74256-15-8 } & \text { 2-(Perfluoroisotridecyl) ethyl methacrylate } & \\ \text { CAS 94158-64-2 } & \text { 2-(Perfluoroisopentadecyl) ethyl methacrylate } & \\ \text { CAS 94158-65-3 } & \text { 2-(Perfluoroisoheptadecyl) ethyl methacrylate } & \end{array}$

Other acrylates

CAS 24407-09-8 3-Perfluoroisononyl-2-hydroxypropyl acrylate

CAS 16083-87-7 3-Perfluoroisotridecyl-2-hydroxypropyl acrylate

CAS 16083-78-6 3-Perfluoroisoheptadecyl-2-hydroxypropyl acrylate

\section{Fluorotelomer phosphates}

\begin{tabular}{lll}
\hline CAS 94200-54-1 & 14:2 Fluorotelomer dihydrogen phosphate & PAP \\
CAS 57678-05-4 & 10:2 Fluorotelomer dihydrogen phosphate & PAP \\
CAS 57678-07-6 & 12:2 Fluorotelomer dihydrogen phosphate & PAP \\
CAS 94200-55-2 & 16:2 Fluorotelomer dihydrogen phosphate & PAP \\
CAS 101896-22-4 & Di(9:2 Fluorotelomer) phosphate & diPAP \\
CAS 57677-98-2 & Di(10:2 Fluorotelomer) hydrogen phosphate with 2,2'-iminodiethanol & diPAP \\
CAS 57677-99-3 & Di(12:2 fluorotelomer) hydrogen phosphate & diPAP \\
CAS 57678-00-9 & Di(12:2 Fluorotelomer) hydrogen phosphate with 2,2'-iminodiethanol & diPAP \\
CAS 94291-75-5 & Di(14:2 fluorotelomer) hydrogen phosphate with 2,2'iminodiethanol & diPAP \\
CAS 94291-76-6 & Di(16:2 fluorotelomer) hydrogen phosphate with 2,2'iminodiethanol & diPAP
\end{tabular}




\begin{tabular}{|c|c|c|c|}
\hline CAS $94094-26-5$ & \multicolumn{3}{|c|}{ 4:2 Fluorotelomer $-1,1^{\prime}$-di(tetradecanoic acid)-methyl silane } \\
\hline CAS $61798-68-3$ & \multicolumn{2}{|c|}{$8: 2$ Fluorotelomer pyridinium salt } & C8 Chemical \\
\hline *CAS 78560-45-9 & \multicolumn{2}{|c|}{$6: 2$ Fluorotelomer trichlorosilane } & \\
\hline *CAS 78560-44-8 & \multicolumn{2}{|c|}{$8: 2$ Fluorotelomer trichlorosilane } & C8 chemical \\
\hline CAS 83048-65-1 & \multicolumn{2}{|c|}{$8: 2$ Fluorotelomer trimethoxysilane } & C8 chemical \\
\hline CAS $67846-66-6$ & \multicolumn{3}{|c|}{$\begin{array}{l}\text { Sodium C-ethyl [2-(sulfonato-thio)ethyl]-(3,3,4,4,5,5,6,6,7,7,8,- } \\
\text { 8,8-tridecafluorooctyl) carbamate }\end{array}$} \\
\hline \multicolumn{4}{|c|}{ Polymers, CAS numbers } \\
\hline \multicolumn{2}{|c|}{$479029-28-2$} & $56372-23-7$ & $65530-65-6$ \\
\hline \multicolumn{2}{|c|}{$68298-79-3$} & $68298-80-6$ & $68298-81-7$ \\
\hline \multicolumn{2}{|c|}{$\begin{array}{l}\text { 45080-67-0 Polyfox PF-156A, } \\
\text { polymer with C3-fluoro chain, floor } \\
\text { polish }\end{array}$} & $\begin{array}{l}\text { 452080-64-7 Polyfox PF-136A, polymer } \\
\text { with C3-fluoro chain, floor polish }\end{array}$ & $\begin{array}{l}65545-80-4 Z \text { Zonyl FSO } \\
100, \text { wetting agent }\end{array}$ \\
\hline \multicolumn{2}{|l|}{ 69991-61-3 } & 65530-61-2 Zonyl UR & 65530-60-1 Zonyl BA-N \\
\hline \multicolumn{2}{|c|}{$\begin{array}{l}\text { 123171-68-6 Zonyl }{ }^{\oplus} \text { FSK, wetting } \\
\text { agent }^{28}\end{array}$} & $\begin{array}{l}\text { 135228-60-3 Zonyl 9155, carpet protec- } \\
\text { tor }\end{array}$ & $\begin{array}{l}\text { 203743-03-7 Foraper- } \\
\mathrm{le}^{\circledR} 225 \text {, repellent }\end{array}$ \\
\hline \multicolumn{2}{|c|}{$\begin{array}{l}\text { 60164-51-4 polyperfluoropropyl } \\
\text { ether, Zonyl PFPE, lubricant }\end{array}$} & 65605-70-1 Zonyl Acrylate N-Li & $\begin{array}{l}65605-58-5 \text { Zonyl G } \\
\text { Fabric Protector }\end{array}$ \\
\hline \multicolumn{2}{|c|}{ 65530-62-3 Zonyl UR } & 65530-64-5 Zonyl 9027, repellant & $\begin{array}{l}65530-63-4 \text { Zonyl 9027, } \\
\text { repellant }\end{array}$ \\
\hline \multicolumn{2}{|c|}{$\begin{array}{l}\text { 65530-69-0 Zonyl FSA, wetting } \\
\text { agent }\end{array}$} & 6530-82-7 Zonyl TELB-L67 & $\begin{array}{l}65530-74-7 \text { Zonyl 9027, } \\
\text { repellent }\end{array}$ \\
\hline \multicolumn{4}{|c|}{ 71215-70-8 (Zonyl PFHEI) } \\
\hline
\end{tabular}

\section{Undefined mixtures, CAS numbers}

\begin{tabular}{llll}
\hline $68081-83-4$ & $68140-18-1$ & $68140-19-2$ & $68140-20-5$ \\
$68187-25-7$ & $68187-47-3$ & $68140-21-6$ & $68391-08-2$ (Zonyl BA-LD) \\
$68391-09-3$ & $68412-68-0$ & $68412-69-1$ & $70983-60-7$ \\
$71608-60-1$ & $74499-44-8$ & $84238-62-0$ & $85631-54-5$ \\
$86508-42-1$ & $90622-43-8$ & $91032-01-8$ & $91081-99-1$ \\
$101940-12-9$ & $161074-58-4$ & $479029-28-2$ & $68081-83-4$ \\
$68140-18-1$ & $68140-19-2$ & $68140-20-5$ & $68140-21-6$ \\
$68187-24-6$ & $68187-25-7$ & $68187-42-8$ & $68187-47-3$ \\
$68188-12-5$ (Zonyl TELB) & $68333-92-6$ & $68391-08-2$ & $68391-09-3$ \\
$68412-68-0$ & $68412-69-1$ & $68608-13-9$ & $68954-01-8$ \\
$70983-60-7$ & $72968-38-8$ & $74499-44-8$ & $84238-62-0$ \\
$85631-40-9$ & $85631-54-5$ & $85681-64-7$ & $85995-90-0$ \\
$85995-91-1$ & $86508-42-1$ & $90481-10-0$ & $90622-43-8$ \\
$90622-71-2$ & $90622-99-4$ & $91032-01-8$ & $91081-09-3$ \\
$91081-99-1$ & $91648-32-7$ & $91770-74-0$ & $91770-94-4$ \\
$92129-34-5$ & $92332-25-7$ & $92332-26-8$ & $93062-53-4$ \\
$93572-72-6$ & $94095-37-1$ & $94166-88-8$ & $95370-51-7$ \\
$97660-44-1$ & $98219-29-5$ & $98561-40-1$ & \\
\hline
\end{tabular}

${ }^{28}$ MST 2008 Report. 



\section{Appendix C - List of contacted companies/institutions}

\begin{tabular}{|c|c|c|}
\hline Company name & Country & Application/use \\
\hline DuPont & USA & Producer \\
\hline DuPont & France & Producer \\
\hline $3 \mathrm{M}$ & USA & Producer \\
\hline $3 \mathrm{M}$ & Belgium & Producer \\
\hline Plastics Europe & Belgium & Trade association \\
\hline Fluorocouncil & USA & \\
\hline KEMI (Swedish Chemicals Agency) & Sweden & Authority \\
\hline NYCO & Norway, France & Aviation hydraulic fluids \\
\hline Solberg & & Fire fighting foams \\
\hline Dr. Sthamer & Switzerland & Fire fighting foams \\
\hline Kiesow Dr. Brinkmann & Germany & Metal plating \\
\hline Atotech & Sweden & Metal plating \\
\hline McDermid & Sweden & Metal plating \\
\hline Wolfgang Podestà & Germany & Trade association; Metal plating \\
\hline Statoil & Norway & Chemically driven oil production \\
\hline Clariant & Germany & Impregnation \\
\hline Daikin & & Impregnation \\
\hline Rudolf Chemie & Germany & Impregnation \\
\hline Huntsman & Germany; Sweden & Impregnation \\
\hline Everest & & Impregnation \\
\hline Dickson & Sweden & Impregnation \\
\hline Helly Hansen & Norway & Impregnation \\
\hline Emballageindustrien (Dansk Industri) & Denmark & Trade association; Food contact materials \\
\hline Valsemøllen & Denmark & Food contact materials \\
\hline Sonax & Germany & Impregnation \\
\hline Melvo & Germany & Impregnation \\
\hline NixWax & UK & Impregnation \\
\hline Dansk Industri & Denmark & Trade association; Coatings \\
\hline STAMI & Norway & Authority \\
\hline Omnova Solutions & USA & Cleaning products \\
\hline Dansk Mode \& Textil & Denmark & Trade associations; Textiles; Impregnation \\
\hline $\begin{array}{l}\text { Tukes (Finnish Safety and Chemicals } \\
\text { Agency) }\end{array}$ & Finland & Authority \\
\hline $\begin{array}{l}\text { Umhverfisstofnun (The Environment } \\
\text { Agency of Iceland) }\end{array}$ & Iceland & Authority \\
\hline Plastindustrien i Danmark & Denmark & Trade asscociation; Plastics \\
\hline
\end{tabular}




\begin{tabular}{lll}
\hline Company name & Country & Application/use \\
\hline The Icelandic Industry Association & Iceland & Trade association - industry \\
ITEK, Dansk Industri & Denmark & Trade association; Electronics \\
Finish Printing Ink Association & Finland & Trade association; Coatings \\
Tikkurila & Finland & Coatings \\
The Federation of Finnish Textiles and & Finland & Trade association; Textiles \\
Clothing Industries & & \\
Finnish Forest Industries & Finland & Trade association; Packaging/paper \\
Finnish Plastics Industries Federation & Finland & Trade association; Plastics \\
The Federation of Finnish Technology & Finland & Trade association; Electronics \\
Industries & & \\
Selected trade associations in Norway & & Trade associations \\
Selected trade associations in Sweden & & Trade associations \\
Mondi & Finland & Paper and packaging \\
Solvay Specialty Polymers & Italien & Producer \\
AGC Chemicals Europe & Netherlands & Producer \\
Mitsubishi International GmBH & Germany & Distributor \\
HOPI POPI & Czech Republic & Food paper (popcornbags) \\
\hline
\end{tabular}

\section{Questionaire sent for the contacted companies}

Questions - Nordic study on use and emission of per- and polyfluorinated substances

In short we would like information about the use of certain groups of per- and polyfluorinated substances (see item no. 1) used in the Nordic countries. Any information is welcome, however, the more detailed the better.

1. Mapping of polyfluorinated chemicals in the Nordic countries Please provide information about the use of these groups of fluorinated compounds within your industry. Are the substances used - yes or no?

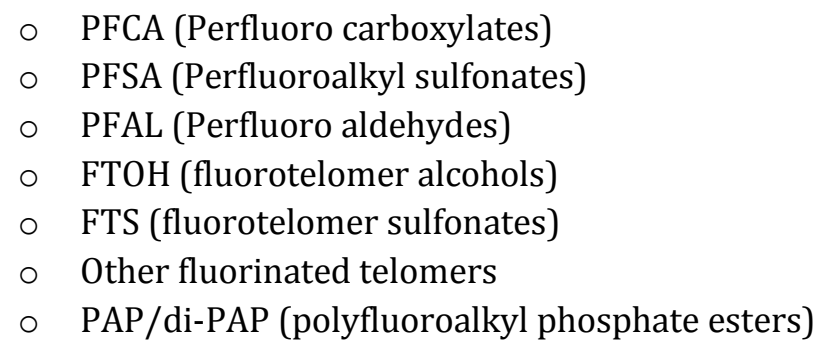

2. Mapping of polyfluorinated chemicals in the Nordic countries detailed information

Please provide more detailed information about the use of the above mentioned groups of fluorinated compounds:

○ information about quantities

○ information about application/uses 
- information about producers

- information about downstream users and traders in the Nordic countries for the uses within your industry

- information about trade names for products containing any of these substances

For those uses that are not relevant for the Nordic market, please, make a remark for these uses.

3. Identity and properties

Please provide the following information for the above PFCs in question that are relevant for the Nordic market:

- Chemical name

- CAS number

- Trade name

- Concentration

- The corresponding uses

4. Efficacy and availability

Please provide, if available, for these PFCs any information on:

- Performance

- Benefits

- Costs and limitations

- Availability on the Nordic market

Thank you very much for your help! 



\section{Appendix D - Commercial PFC products and brands on the market}

\begin{tabular}{|c|c|}
\hline PFC product & References \\
\hline DuPont Capstone ${ }^{\oplus}$ Fluorosurfactants & $\begin{array}{l}\text { http://www2.dupont.com/Capstone/en_US/assets/downloads/ } \\
\text { capstone_1157.pdf }\end{array}$ \\
\hline Chemguard Fluorosurfactants & $\begin{array}{l}\text { http://www.chemguard.com/fire-suppression/catalog/ } \\
\text { foam-concentrates/aqueous-film-forming-foam-afff/ }\end{array}$ \\
\hline Dynax Fluorosurfactants & $\begin{array}{l}\text { http://www.dynaxcorp.com/resources/pdf/2009/ } \\
\text { dx5022.bul-rev0909.pdf }\end{array}$ \\
\hline $\begin{array}{l}\text { Solberg high hydrocarbon foaming agent } \\
\text { concentration (Fluorine Free) }\end{array}$ & $\begin{array}{l}\text { http://www.solbergfoam.com/Foam-Concentrates/ } \\
\text { RE-HEALING }{ }^{\text {TM }} \text {-Foam.aspx }\end{array}$ \\
\hline
\end{tabular}

Table 2. A selection of metal plating mist suppressant products on the market ${ }^{30}$

\begin{tabular}{|c|c|}
\hline PFC product & References \\
\hline 3M Mist Suppressants & $\begin{array}{l}\text { http://solutions.3m.com/wps/portal/3M/en_US/ } \\
\text { Energy-Advanced/Materials/Products/Acid_Mist_Suppressants/ }\end{array}$ \\
\hline Atotech Mist Suppressants & $\begin{array}{l}\text { http://www.atotech.com/products/general-metal-finishing/ } \\
\text { functional-chrome-plating/fumetrolr-21-If.html }\end{array}$ \\
\hline Enthone Mist Suppressants & $\begin{array}{l}\text { http://enthone.com/en/Industries/Industrial_Finishes/ } \\
\text { Technology_Selector/Products/ } \\
\text { ENTHONE_PFOS-Free_Solutions.aspx }\end{array}$ \\
\hline McDermid Mist Suppressants & $\begin{array}{l}\text { http://industrial.macdermid.com/cms/engineering/hardchrome/ } \\
\text { index.shtml }\end{array}$ \\
\hline Hunter Chemical Fume Suppressants & http://www.hunterchem.com/metal-finishing-Cr.html \\
\hline Kiesow Dr. Brinkmann & $\begin{array}{l}\text { http://www.kiesow.org/aktuelles/aktuelles/article/ } \\
\text { proquel-of-mit-grossem-erfolg/ }\end{array}$ \\
\hline
\end{tabular}

29 Personal information from the Fluorocouncil.

${ }^{30}$ Personal information from the Fluorocouncil and from producers/suppliers of mist

suppressants 
Table 3. A selection of dirt- and water repellent ( DWR) products on the market ${ }^{31}$

\begin{tabular}{|c|c|}
\hline PFC product & References \\
\hline Everest Water Repellant Finishes & $\begin{array}{l}\text { http://www.everest.com.tw/_english/00_site/01_edit.aspx?MI } \\
D=87 \& S I D=118 \& T I D=125\end{array}$ \\
\hline Maflon Leather Fluorosurfactants & http://www.maflon.com/index.php/fluoropolymers.html \\
\hline $\begin{array}{l}\text { Perfluorobutane sulfonamido-based } \\
\text { products } \\
\text { Scotchgard }^{\otimes}\end{array}$ & $\begin{array}{l}\text { http://www.scotchgard.com/wps/portal/3M/en_US/NAScotchg } \\
\text { ard/Global/ }\end{array}$ \\
\hline $\begin{array}{l}\text { Fluorinated oxetane-based products } \\
\text { Polyfox }{ }^{\circledast}\end{array}$ & http://www.omnova.com/products/chemicals/PolyFox.aspx \\
\hline $\begin{array}{l}\text { Wacker Silicone-based Dirt and Water } \\
\text { Repellants }\end{array}$ & $\begin{array}{l}\text { http://www.wacker.com/cms/media/publications/ } \\
\text { downloads/6304_EN.pdf }\end{array}$ \\
\hline AsahiGuard & http://www.asahiguard.jp/eng/ \\
\hline \multicolumn{2}{|c|}{ Short-chain fluorotelomer-based products - Finishing Agents } \\
\hline Nuva ${ }^{\circledR}$ finishing agents & $\begin{array}{l}\text { http://www.textiles.clariant.com/C12571C400483A78/ } \\
\text { vwWebPagesByID/ABCE5BDE71BE7555C12572AC0049E92D }\end{array}$ \\
\hline Unidyne $^{\circledR}$ finishing agents & $\begin{array}{l}\text { http://www.daikin-america.com/products/ProductGrades/ } \\
\text { default.aspx?ApplicationID=\&IndustrylD=\&MyDaikin= } \\
\text { \&productgradeid=73 }\end{array}$ \\
\hline Rudolf Finishing Agents & $\begin{array}{l}\text { http://www.rudolf.de/products/ } \\
\text { details-brochure.htm?year=2010\&ri=201005 }\end{array}$ \\
\hline Oleophobol ${ }^{\circledR}$ Finishing Agents & $\begin{array}{l}\text { http://www2.dupont.com/Capstone/en_US/assets/downloads/ } \\
\text { Capstone_Oleophobol_Detail_Chart_ProductsForTextiles_K- } \\
\text { 25183_CapstoneforTeflon_FINAL_22february2011.pdf }\end{array}$ \\
\hline
\end{tabular}

Table 4. A selection of paper and packaging impregnation products on the market ${ }^{32}$

\begin{tabular}{ll}
\hline PFC product & References \\
\hline DuPont Capstone ${ }^{\oplus}$ Fluorosurfactants & $\begin{array}{l}\text { http://www2.dupont.com/Capstone/en_US/uses_apps/ } \\
\text { paper_packaging/paper_packaging.html } \\
\text { http://www.solvayplastics.com/sites/solvayplastics/EN } \\
\text { /specialty_polymers/Fluorinated_Fluids/Pages/Solvera_PFPE.aspx }\end{array}$ \\
Solvay PFPE specialty polymers & http://www.asahiguard.jp/eng/ \\
\hline
\end{tabular}

31 Personal information from the Fluorocouncil.

32 Personal information from the Fluorocouncil. 
Table 5. A selection of coating agents on the market ${ }^{33}$

\begin{tabular}{|c|c|}
\hline PFC product & References \\
\hline DuPont Capstone ${ }^{\circledast}$ Fluorosurfactants & $\begin{array}{l}\text { http://www2.dupont.com/Capstone/en_US/uses_apps/ } \\
\text { Fluorosurfactants/paints_coatings.html }\end{array}$ \\
\hline Chemguard Fluorosurfactants & $\begin{array}{l}\text { http://www.chemguard.com/specialty-chemicals/ } \\
\text { lodyne-connections.htm }\end{array}$ \\
\hline Dynax Fluorosurfactants & http://www.dynaxcorp.com/technology/coating.html \\
\hline 3M Fluorosurfactants & $\begin{array}{l}\text { http://solutions.3m.com/wps/portal/3M/en_US/ } \\
\text { Energy-Advanced/Materials/Industry_Solutions/ } \\
\text { Paints-Coatings/Novec/ }\end{array}$ \\
\hline Maflon Fluorosurfactants & $\begin{array}{l}\text { http://www.maflon.com/index.php/ } \\
\text { fluorosurfactant-products-and-applications.html }\end{array}$ \\
\hline 3M Aqueous Fluorinated Polyurethane & $\begin{array}{l}\text { http://multimedia.3m.com/mws/mediawebserver?mwsld= } \\
\text { 66666UF6EVsSyXTtMXf6LXfXEVtQEVs6EVs6EVs6E666666-- } \\
\text { \&fn=prodinfo_src220.pdf }\end{array}$ \\
\hline Byk Chemie Additives & http://www.byk.com/en/press-events/new-additives.html \\
\hline Tego Siloxane based Surfactants & $\begin{array}{l}\text { http://www.tego.de/sites/dc/Downloadcenter/Evonik/ } \\
\text { Product/Tego/en/Technical-Papers-Additives/ } \\
\text { article-multifuctional-siloxane-based-gemini-surfactatng- } \\
\text { tego-twin-4000-e.pdf }\end{array}$ \\
\hline Air Products Hydrocarbon Surfactants & $\begin{array}{l}\text { http://www.airproducts.com/ /media/Files/PDF/industries/ } \\
\text { paints-coatings-surfynol-surfactants-multifunctional- } \\
\text { problem-solvers-waterborne.ashx }\end{array}$ \\
\hline Fluorinated oxetane-based products Polyfox ${ }^{\oplus}$ & http://www.omnova.com/products/chemicals/PolyFox.aspx \\
\hline Diederich Siloxane additives & $\begin{array}{l}\text { http://www.diedrichtechnologies.com/ } \\
\text { Water-Repellents-3.php }\end{array}$ \\
\hline
\end{tabular}

${ }^{33}$ Personal information from the Fluorocouncil 

Appendix E - Data contributions

to "Mapping of uses and

applications of PFCs on the

Nordic market" 
Table 1. FTOHs, FTSs, PFSAs and PFOSA in comsumer products (excluding PFCAs)

\begin{tabular}{|c|c|c|c|c|c|c|c|c|c|c|c|c|c|c|c|c|}
\hline Reference & Data & Country & Product type & Usage & FTO & & & & FTS & & & \multicolumn{3}{|c|}{ Sulfonates } & & \\
\hline Dinglasan-Panlilio MJA & A.D. & n.i. & Polyfox-L-diol & C.P. & $4: 2$ & $6: 2$ & $8: 2$ & $10: 2$ & & & $x$ & & & & & \\
\hline Dinglasan-Panlilio MJA & A.D. & n.i. & Teflon Advance & C.P. & & $6: 2$ & $8: 2$ & $10: 2$ & & & $x$ & & & & & \\
\hline $\begin{array}{l}\text { Dinglasan-Panlilio MJA, } \\
\text { DuPONT } 3\end{array}$ & A.D. & DuPont & Zonyl FSO 100 & C.P. & & $6: 2$ & $8: 2$ & $10: 2$ & & & & & & & & \\
\hline Dinglasan-Panlilio MJA & A.D. & DuPont & Zonyl FSE & C.P. & & $6: 2$ & $8: 2$ & $10: 2$ & & & $x$ & & & & & \\
\hline Dinglasan-Panlilio MJA & A.D. & Canada & $\begin{array}{l}\text { Motomaster } \\
\text { windshield } \\
\text { washer }\end{array}$ & C.P. & $4: 2$ & $6: 2$ & $8: 2$ & $10: 2$ & & & $x$ & & & & & \\
\hline Dinglasan-Panlilio MJA & A.D. & n.i. & 8:2 Methacrylate & C.P. & & $6: 2$ & $8: 2$ & & & & & & & & & \\
\hline Dinglasan-Panlilio MJA & A.D. & n.i. & Scotchgard & C.P. & & & & & & & $x$ & & & & & \\
\hline Sinclair E & A.D. & n.i. & $\begin{array}{l}\text { Teflon Frying } \\
\text { pans }\end{array}$ & C.P. & & $6: 2$ & $8: 2$ & & & & & & & & & \\
\hline Sinclair E & A.D. & n.i. & $\begin{array}{l}\text { Microwave } \\
\text { popcorn }\end{array}$ & C.P. & & $6: 2$ & $8: 2$ & & & & & & & & & \\
\hline Sinclair E & A.D. & n.i. & $\begin{array}{l}\text { Microwave } \\
\text { popcorn } \\
\text { packing paper }\end{array}$ & C.P. & & $6: 2$ & $8: 2$ & & & & & & & & & \\
\hline Herzke D & A.D. & Norway & Paint & C.P. & & & & & & & & & $\mathrm{PFHXS}$ & PFHpS & & \\
\hline Herzke D & A.D. & Norway & AFFF* & I.U. & & $6: 2$ & $8: 2$ & $10: 2$ & $6: 2$ & $8: 2$ & & PFBS & PFHXS & PFHpS & PFDcS & PFOSA \\
\hline Herzke D & A.D. & Norway & $\begin{array}{l}\text { Waterproofing } \\
\text { agents }\end{array}$ & C.P. & & $6: 2$ & $8: 2$ & $10: 2$ & & & & PFBS & & & & \\
\hline Herzke D & A.D. & Norway & РCB & I.U. & & & & & & & & & PFHXS & & & \\
\hline Herzke D & A.D. & Norway & Coated fabrics & C.P. & & $6: 2$ & $8: 2$ & $10: 2$ & $6: 2$ & & & PFBS & PFHXS & & & \\
\hline Herzke D & A.D. & Norway & Non-stick ware & C.P. & & $6: 2$ & & $10: 2$ & & & & PFBS & PFHXS & & & \\
\hline Berger & A.D. & n.i. & Textile & C.P. & & & & & & & & & & & & \\
\hline Berger & A.D. & n.i. & textile & C.P. & & & & & & & & & & & & \\
\hline
\end{tabular}

C.P.: Consumer products; I.U.: Industrial use; X: NMeFOSE; * Still usage of some products. 


\section{Table 2. PFCAs in consumer products}

\begin{tabular}{|c|c|c|c|c|c|c|c|c|c|c|c|c|c|c|}
\hline References & Data & Country & Product type & PFCAs & & & & & & & & & & \\
\hline Dinglasan-Panlilio MJA & A.D. & n.i. & Polyfox-L-diol & & & & & & & & & & & \\
\hline Dinglasan-Panlilio MJA & A.D. & n.i. & Teflon Advance & & & & & & & & & & & \\
\hline $\begin{array}{l}\text { Dinglasan-Panlilio MJA, } \\
\text { DuPONT } 3\end{array}$ & A.D. & DuPont & Zonyl FSO 100 & & & & & & & & & & & \\
\hline Dinglasan-Panlilio MJA & A.D. & DuPont & Zonyl FSE & & & & & & & & & & & \\
\hline Dinglasan-Panlilio MJA & A.D. & Canada & $\begin{array}{l}\text { Motomaster windshield } \\
\text { washer }\end{array}$ & & & & & & & & & & & \\
\hline Dinglasan-Panlilio MJA & A.D. & n.i. & 8:2 Meth-acrylate & & & & & & & & & & & \\
\hline Dinglasan-Panlilio MJA & A.D. & n.i. & Scotchgard & & & & & & & & & & & \\
\hline Sinclair E & A.D. & n.i. & Teflon Frying pans & & & & & & & & & & & \\
\hline Sinclair E & A.D. & n.i. & Microwave popcorn & & & & & & & & & & & \\
\hline Sinclair E & A.D. & n.i. & $\begin{array}{l}\text { Microwave popcorn } \\
\text { packing paper }\end{array}$ & & PFPA & & PFHpA & PFNA & PFDcA & PFUnDA & PFDoDA & & & \\
\hline Herzke D & A.D. & Norway & Paint & PFBA & & & & & & & & & & \\
\hline Herzke D & A.D. & Norway & AFFF* & PFBA & PFPA & PFHXA & & & PFDcA & & PFDoA & & & \\
\hline Herzke D & A.D. & Norway & Waterproofing agents & PFBA & & PFHXA & PFHpA & PFNA & PFDcA & PFUnDA & PFDoA & & PFTeA & \\
\hline Herzke D & A.D. & Norway & PCB & PFBA & & & & & & & & & & \\
\hline Herzke D & A.D. & Norway & Coated fabrics & & PFPA & PFHXA & PFHpA & PFNA & PFDcA & & PFDoA & & & \\
\hline Herzke D & A.D. & Norway & Non-stick ware & PFBA & & & & & & & & & & \\
\hline Berger & A.D. & n.i. & Textile & & & PFHXA & PFHpA & PFNA & PFDcA & PFUnDA & PFDoA & PFTriA & PFTeA & PFPDA \\
\hline Berger & A.D. & n.i. & Textile & PFBA & & PFHXA & PFHpA & PFNA & PFDcA & PFUnDA & PFDoA & & PFTeA & \\
\hline
\end{tabular}


Table 3. PFCs in consumer productions, (other than stated in table 1 and 2)

\begin{tabular}{|c|c|c|c|c|c|c|c|c|c|c|c|}
\hline Reference & & $\begin{array}{l}\text { Prod. } \\
\text { Country }\end{array}$ & Product type & Usage & type & $\begin{array}{l}\text { Usage } \\
\text { still? }\end{array}$ & & & & & \\
\hline Vorob'ev SI & A.D. & Japan & Fluosol-DA 20\% & Blood substitutes & perfluorocarbon emulsions & No & PFD & PFTPA & & & \\
\hline Vorob'ev SI & A.D. & Japan & Fluosol-DA 35\% & Blood substitutes & perfluorocarbon emulsions & No & PFD & PFTPA & & & \\
\hline Vorob'ev SI & A.D. & Russia & Perftoran & Blood substitutes & perfluorocarbon emulsions & No & PFD & & PFMCP & & \\
\hline Vorob'ev SI & A.D. & Russia & Ftorosan & Blood substitutes & perfluorocarbon emulsions & Yes & PFD & & PFMCP & & \\
\hline Vorob'ev SI; Castro IC & A.D. & USA & Oxygent & Blood substitutes & perfluorocarbon emulsions & Yes & & & & PFOB & PFDB \\
\hline $3 M$ MSDS & P.I. & n.i. & $\begin{array}{l}\text { Scotchgard Carped \& } \\
\text { rug protector } \\
(1023-17 N)\end{array}$ & surfactant & n.i. & n.i. & $\begin{array}{l}\text { Fluor } \\
\text { Uretl } \\
\text { (trad }\end{array}$ & $\begin{array}{l}\text { chemical } \\
\text { ine } \\
\text { secret) }\end{array}$ & & & \\
\hline $3 \mathrm{M}$ & P.I. & n.i. & Dyneon & $\begin{array}{l}\text { Industrial } \\
\text { processing }\end{array}$ & Polytetrafluoroethylene & n.i. & & & & & \\
\hline $3 M$ & P.I. & n.i. & Fluorinet & Electonic liquid & Perfluoro compounds $\left(>\mathrm{C}_{15}\right)$ & n.i. & & & & & \\
\hline $3 \mathrm{M}$ & P.I. & n.i. & Novec 1230 & $\begin{array}{l}\text { Fire extinguishing } \\
\text { agent }\end{array}$ & CF 3 CF $2 C(=O) C F(C F 3) 2$ & n.i. & & & & & \\
\hline $3 \mathrm{M}$ & P.I. & n.i. & $\begin{array}{l}\text { Novec } \text { TM Fluorosurfac- }^{\text {tants FC-4432 }}\end{array}$ & $\begin{array}{l}\text { Paints and } \\
\text { coatings }\end{array}$ & PFBS & n.i. & & & & & \\
\hline Daikin & P.I. & $\begin{array}{l}\text { Japan, } \\
\text { EU, USA }\end{array}$ & $\begin{array}{l}\text { Neoflon-PCTFE Flu- } \\
\text { oropolymer }\end{array}$ & n.i. & Poly(chlorotrifluoroethylene) & & & & & & \\
\hline Daikin & P.I. & $\begin{array}{l}\text { Japan, } \\
\text { EU, USA }\end{array}$ & $\begin{array}{l}\text { Neoflon-PFA Fluoro- } \\
\text { telomer }\end{array}$ & n.i. & $\begin{array}{l}\text { Perfluorovinylpropyl ether- } \\
\text { tetrafluoroethylene }\end{array}$ & & & & & & \\
\hline Daikin & P.I. & $\begin{array}{l}\text { Japan, } \\
\text { EU, USA }\end{array}$ & OPTOOL & $\begin{array}{l}\text { Prevent finger- } \\
\text { print marking }\end{array}$ & Perfluorohexane & & & & & & \\
\hline Daikin & P.I. & $\begin{array}{l}\text { Japan, } \\
\text { EU, USA }\end{array}$ & UNIDYNE & Fluoro coating & n.i. & & & & & & \\
\hline Daikin & P.I. & $\begin{array}{l}\text { Japan, } \\
\text { EU, USA }\end{array}$ & DAIFREE & Fluoro coating & n.i. & & & & & & \\
\hline Daikin & P.I. & $\begin{array}{l}\text { Japan, } \\
\text { EU, USA }\end{array}$ & $\begin{array}{l}\text { Polyflon PTFE- } \\
\text { Fluoropolymer }\end{array}$ & Teflon & polytetrafluoroethylene & & & & & & \\
\hline Daikin & P.I. & $\begin{array}{l}\text { Japan, } \\
\text { EU, USA }\end{array}$ & Dai-El & Elastomer & $\begin{array}{l}\text { vinylidenefluoride/ } \\
\text { hexafluoropropylene } \\
\text { copolymer }\end{array}$ & & & & & & \\
\hline
\end{tabular}




\begin{tabular}{|c|c|c|c|c|c|c|c|c|c|}
\hline Reference & & $\begin{array}{l}\text { Prod. } \\
\text { Country }\end{array}$ & Product type & Usage & type & $\begin{array}{l}\text { Usage } \\
\text { still? }\end{array}$ & & & \\
\hline Asahi Glass Company & P.I. & & Fluon PTFR_E & Polymers & Polytetrafluoroethylene & & & & \\
\hline Dow Corning & P.I. & & Molykote & Grease & PFPE, PTFE & & & & \\
\hline DuPont 1,2 & P.I. & USA & Teflon Advanced & $\begin{array}{l}\text { Carpet and } \\
\text { upholstery } \\
\text { protection }\end{array}$ & n.i. & n.i. & $\begin{array}{l}\text { Fluor } \\
\text { cal di } \\
\text { in wa }\end{array}$ & $\begin{array}{l}\text { chemi- } \\
\text { ersion } \\
\text { er }\end{array}$ & Partially fluorinated aliphatic polyurethane \\
\hline DuPont & P.I. & USA & Viton & Elastomer & $\begin{array}{l}\text { Hexafluoropropene } \\
\text { polymer }\end{array}$ & & & & \\
\hline DuPont & P.I. & USA & Kalrez & Fire fighting & $\begin{array}{l}\text { Perfluoroalkylpolyether, } \\
\text { polytetrafluoroethylene }\end{array}$ & & & & \\
\hline DuPont & P.I. & USA & Krytox & Lubricant & $\begin{array}{l}\text { Polyhexafluoropropylene } \\
\text { oxide, PFPE, Perfluoroalky- } \\
\text { lether, PTFE }\end{array}$ & & & & \\
\hline Moe M; Zaggia A & A.D. & & ForaFac (DuPont) & $\begin{array}{l}\text { Fire extinguishing } \\
\text { surfactant }\end{array}$ & (CF2)nC9H19 fluorotelomer & & & & \\
\hline Key BD & P.I. & n.i. & monofluoroacetic acid & pesticide & $\mathrm{CH} 2 \mathrm{FCO} 2 \mathrm{H}$ & n.i. & & & \\
\hline Key BD & P.I. & n.i. & Trifluoroacetic acid & Reagent & $\mathrm{CF} 2 \mathrm{CO} 2 \mathrm{H}$ & n.i. & & & \\
\hline Key BD & P.I. & n.i. & $\begin{array}{l}\text { trifluoromethanesulfo- } \\
\text { nic acid }\end{array}$ & catalyst/reagent & $\mathrm{CF} 3 \mathrm{SO}_{3} \mathrm{H}$ & n.i. & & & \\
\hline Key BD & P.I. & n.i. & $\begin{array}{l}1 \mathrm{H}, 1 \mathrm{H}, 2 \mathrm{H}, 2 \mathrm{Hperfluoro} \\
\text { octanesulfonic acid }\end{array}$ & surfactant & $\mathrm{C} 6 \mathrm{~F} 13 \mathrm{CH} 2 \mathrm{CH} 2 \mathrm{SO} 3 \mathrm{H}$ & n.i. & & & \\
\hline Key BD & P.I. & n.i. & $\begin{array}{l}\mathrm{N} \text {-acetic-N-ethyl } \\
\text { perfluorooctane } \\
\text { sulfonamide }\end{array}$ & surfactant & $\begin{array}{l}\mathrm{C} 8 \mathrm{~F} 17 \mathrm{SO} 2 \mathrm{~N}(\mathrm{CH} 2 \mathrm{COOH}) \\
(\mathrm{CH} 2 \mathrm{CH} 3)\end{array}$ & n.i. & & & \\
\hline Key BD & P.I. & n.i. & sulfuramid & insecticide & $\mathrm{C} 8 \mathrm{~F} 17 \mathrm{SO} 2 \mathrm{NH}(\mathrm{CH} 2 \mathrm{CH} 3)$ & n.i. & & & \\
\hline Key BD & P.I. & n.i. & polytetrafluoroethylene & teflon & (-(CF2CF2)n-) & n.i. & & & \\
\hline Key BD & P.I. & n.i. & Perfluoropolyether & lubricant & $(-($ CF(CF3)CF2O)n-) & n.i. & & & \\
\hline Key BD & P.I. & n.i. & Zonyl alcohol & surfactant & $\mathrm{C} 8 \mathrm{~F} 17 \mathrm{CH} 2 \mathrm{CH} 2 \mathrm{OH}$ & n.i. & & & $\begin{array}{l}8: 2- \\
\text { FTOH }\end{array}$ \\
\hline Yang Z & P.I. & n.i. & Oxycyte & Blood substitutes & perfluorocarbon emulsions & n.i. & & & \\
\hline Castro IC & P.I. & n.i. & Fluosol & Blood substitutes & perfluorocarbon emulsions & n.i. & PFD & PFTPA & \\
\hline Castro IC & P.I. & n.i. & Oxypherol & Blood substitutes & perfluorocarbon emulsions & n.i. & & PFTPA & \\
\hline
\end{tabular}




\begin{tabular}{|c|c|c|c|c|c|c|c|c|c|}
\hline Reference & & $\begin{array}{l}\text { Prod. } \\
\text { Country }\end{array}$ & Product type & Usage & type & $\begin{array}{l}\text { Usage } \\
\text { still? }\end{array}$ & & & \\
\hline Castro IC & P.I. & n.i. & Perftoran & Blood substitutes & perfluorocarbon emulsions & n.i. & PFD & & \\
\hline Castro IC & P.I. & n.i. & Oxyfluor & Blood substitutes & perfluorocarbon emulsions & n.i. & & PFDCO & \\
\hline Castro IC & P.I. & n.i. & Oxycyte & Blood substitutes & perfluorocarbon emulsions & n.i. & & & TBPCH \\
\hline Castro IC & P.I. & Columbia & Columbian emulsion & Blood substitutes & perfluorocarbon emulsions & n.i. & & PFOB & \\
\hline Castro IC & P.I. & France & French emulsion & Blood substitutes & perfluorocarbon emulsions & n.i. & & PFOB & \\
\hline Gelest & P.I. & n.i. & SiBRID FCS 331 & $\begin{array}{l}\text { Skin care product } \\
\text { ingredient } \\
\text { (cosmetics) }\end{array}$ & n.i. & n.i. & $\begin{array}{l}\text { tetrafluoro- } \\
\text { ethylene }\end{array}$ & fluorinated dimethyl fluid & \\
\hline Solvay plastics & P.I. & n.i. & Fomblin PFPE & $\begin{array}{l}\text { Lubricants/ } \\
\text { oils/grease/ } \\
\text { surfactant }\end{array}$ & PFPE & & & & \\
\hline Solvay plastics & P.I. & n.i. & Fomblin HC PFPE & $\begin{array}{l}\text { Personal care } \\
\text { products }\end{array}$ & PFPE & & & & \\
\hline Solvay plastics & P.I. & n.i. & Hyflon & $\begin{array}{l}\text { Wire \& cable } \\
\text { coatings }\end{array}$ & PFA/MFA & & & & \\
\hline Solvay plastics & P.I. & n.i. & Tecnoflon & $\begin{array}{l}\text { Per- } \\
\text { fluoroelastomer }\end{array}$ & Fluoropolyether derivative & & & & \\
\hline Solvay plastics & P.I. & n.i. & Algoflon & $\begin{array}{l}\text { Wire \& cable } \\
\text { coatings }\end{array}$ & PTFE & & & & \\
\hline Solvay plastics & P.I. & n.i. & Fluorolink & Miscellaneous & PFPE & & & & \\
\hline Solvay plastics & P.I. & n.i. & Galden & Electronics & PFPE & & & & \\
\hline Solvay plastics & P.I. & n.i. & Solvera & Paper packaging & n.i. & & & & \\
\hline Hoechst AG & P.I. & Germany & Hostaflon & Teflon & & & & & \\
\hline Hoechst AG & P.I. & Germany & Hostinert & $\begin{array}{l}\text { In electronic } \\
\text { components }\end{array}$ & Perfluorinated liquid & No & & & \\
\hline Penwalt & P.I. & n.i. & Pentel & $\begin{array}{l}\text { Water and soil } \\
\text { repellency to } \\
\text { fabrics }\end{array}$ & Fluorotelomer composition & & & & \\
\hline Miteni & P.I. & Italy & Perflutel RM82 & $\begin{array}{l}\text { Industry and } \\
\text { science }\end{array}$ & Perfluorohexane & & & & \\
\hline
\end{tabular}




\begin{tabular}{|c|c|c|c|c|c|c|}
\hline Reference & & $\begin{array}{l}\text { Prod. } \\
\text { Country }\end{array}$ & Product type & Usage & type & $\begin{array}{l}\text { Usage } \\
\text { still? }\end{array}$ \\
\hline Miteni & P.I. & Italy & Perflutel RM57 & $\begin{array}{l}\text { Industry and } \\
\text { science }\end{array}$ & Perfluoroheptane & \\
\hline NearChimica & P.I. & Italy & Naiguard & Repellant finishes & PTFE & \\
\hline Zaggia A & P.I. & n.i. & PolyFox & $\begin{array}{l}\text { Fire-extinguishing } \\
\text { surfactant }\end{array}$ & $\begin{array}{l}\text { Hydroxyl terminated } \\
\text { Fluoropolyether co-polymer }\end{array}$ & \\
\hline OMNOVA & P.I. & n.i. & X-Cape & Repellants & Fluoropolymer & \\
\hline
\end{tabular}

A.D.: Analytical data, P.I.: Producer information.

Abbreviations used in Table 3

PFD

$\begin{array}{ll}\text { PFTPA } & \text { Perfluorotributylamine }\end{array}$

PFMCP perfluoromethylcyclohexylpiperidine

PFOB $\quad$ Perfluorooctylbromide

Perfluorodecylbromide

PFDCO Perfluorodichlorooctane

TBPCH Tetrabutylperfluorocyclohexane 
Table 4. Geometric mean concentration in home and work related environments indicating human exposure

\begin{tabular}{|c|c|c|c|c|c|c|c|c|c|c|}
\hline & Reference & Jogsten IE & Fracer AJ & Shoeib M (2005) & Shoeib M (2004) & Barber JL & Barber JL & Jahnke A & Jahnke A & Shoeib M (2007) \\
\hline & Country & Spain & USA & Canada & Canada & Norway & Norway & Norway & Norway & Canada \\
\hline & Matrix & Indoor dust & Office air & Indoor air/dust & Indoor air/dust & Indoor air/dust & Indoor air/dust & Indoor air/dust & Indoor air/dust & Indoor air/dust \\
\hline & Year & 2012 & 2012 & 2005 & 2004 & 2007 & 2007 & 2007 & 2007 & 2007 \\
\hline & Unit & $\mathrm{ng} / \mathrm{g}$ & $\mathrm{pg} / \mathrm{m} 3$ & $\mathrm{pg} / \mathrm{m} 3$ & $\mathrm{pg} / \mathrm{m} 3$ & $\mathrm{pg} / \mathrm{m} 3$ & $\mathrm{pg} / \mathrm{m} 3$ & $\mathrm{pg} / \mathrm{m} 3$ & $\mathrm{pg} / \mathrm{m} 3$ & $\mathrm{pg} / \mathrm{m} 3$ \\
\hline \multirow[t]{27}{*}{ Telomers } & 4:2 FTOH & & & & & 114 & $<20$ & & & \\
\hline & 6:2-FTOH & 0.295 & 1,320 & & & 2,990 & $<40$ & 177 & 248 & \\
\hline & 8:2-FTOH & 4.04 & 9,920 & & & 3,424 & $<10$ & 853 & 421 & 2,070 \\
\hline & 10:2-FTOH & 0.76 & 2,850 & & & 3,559 & 13 & 898 & 1,660 & 891 \\
\hline & EtFOSA & $<0.062$ & 17 & 59 & & 6,626 & 7 & 188 & 158 & \\
\hline & MeFOSA & $<0.054$ & 29,1 & & & 6,608 & 6 & & & \\
\hline & EtFOSE & 3.238 & 18,1 & 1,100 & 770 & 5,755 & 76 & 305 & 815 & \\
\hline & MeFOSE & 1.19 & 289 & 1,970 & 2,590 & 6,018 & 763 & 727 & 798 & \\
\hline & & & & 35 & 73 & & & & & \\
\hline & PFBS & 1.028 & & & & & & & & \\
\hline & PFHxS & 1.073 & & & & & & & & \\
\hline & PFDS & $<0.002$ & & & & & & & & \\
\hline & PFBA & 16.35 & & & & & & & & \\
\hline & PFPeA & 0.367 & & & & & & & & \\
\hline & PFHXA & 1.382 & & & & & & & & \\
\hline & PFHpA & 1.63 & & & & & & & & \\
\hline & PFNA & 6.769 & & & & & & & & \\
\hline & PFDA & 9.735 & & & & & & & & \\
\hline & PFUnDA & 3.373 & & & & & & & & \\
\hline & PFDoDA & 3.363 & & & & & & & & \\
\hline & PFTrDA & 10.9086 & & & & & & & & \\
\hline & PFTDA & 0.67 & & & & & & & & \\
\hline & PFOCDA & $<0.46$ & & & & & & & & \\
\hline & 5:3 FTSA & $<2$ & & & & & & & & \\
\hline & 6:2 FTUCA & 0.0107 & & & & & & & & \\
\hline & 8:2 FTUCA & 0.0265 & & & & & & & & \\
\hline & 10:2 FTUCA & $<0.006$ & & & & & & & & \\
\hline
\end{tabular}




\begin{tabular}{|c|c|c|c|c|c|c|c|c|c|c|c|c|c|c|}
\hline & Reference & $\begin{array}{l}\text { Goosey E } \\
\text { (2012) }\end{array}$ & $\begin{array}{l}\text { Goosey E } \\
\text { (2012) }\end{array}$ & $\begin{array}{l}\text { Goosey E } \\
\text { (2011) }\end{array}$ & $\begin{array}{l}\text { Goosey E } \\
\text { (2011) }\end{array}$ & $\begin{array}{l}\text { Goosey E } \\
\text { (2011) }\end{array}$ & $\begin{array}{l}\text { Goosey E } \\
\text { (2011) }\end{array}$ & $\begin{array}{l}\text { Goosey E } \\
\text { (2011) }\end{array}$ & $\begin{array}{l}\text { Goosey E } \\
\text { (2011) }\end{array}$ & $\begin{array}{l}\text { Goosey E } \\
\text { (2011) }\end{array}$ & $\begin{array}{l}\text { Goosey E } \\
\text { (2011) }\end{array}$ & $\begin{array}{l}\text { Goosey E } \\
\text { (2011) }\end{array}$ & $\begin{array}{l}\text { Kim SK } \\
\text { (2012) }\end{array}$ & $\begin{array}{l}\text { Liu W } \\
\text { (2012) }\end{array}$ \\
\hline & Country & UK & UK & UK & UK & Australia & Canada & France & Germany & Kazahkstan & Thailand & USA & Korea & Japan \\
\hline & Matrix & $\begin{array}{l}\text { Indoor } \\
\text { air - } \\
\text { homes }\end{array}$ & $\begin{array}{l}\text { Indoor } \\
\text { air- } \\
\text { offices }\end{array}$ & $\begin{array}{l}\text { Indoor } \\
\text { air/dust } \\
\text {-homes }\end{array}$ & $\begin{array}{l}\text { Indoor } \\
\text { air/dust } \\
\text {-offices }\end{array}$ & $\begin{array}{l}\text { Indoor } \\
\text { air/dust } \\
\text { - homes }\end{array}$ & $\begin{array}{l}\text { Indoor } \\
\text { air/dust } \\
\text { - homes }\end{array}$ & $\begin{array}{l}\text { Indoor } \\
\text { air/dust } \\
\text { - homes }\end{array}$ & $\begin{array}{l}\text { Indoor } \\
\text { air/dust } \\
\text { - homes }\end{array}$ & $\begin{array}{l}\text { Indoor air } \\
\text { /dust - } \\
\text { homes }\end{array}$ & $\begin{array}{l}\text { Indoor } \\
\text { air/dust } \\
\text { - homes }\end{array}$ & $\begin{array}{l}\text { Indoor } \\
\text { air/dust } \\
\text { - homes }\end{array}$ & $\begin{array}{l}\text { Indoor } \\
\text { air }\end{array}$ & $\begin{array}{l}\text { Indoor } \\
\text { air- } \\
\text { homes }\end{array}$ \\
\hline & Year & $\begin{array}{l}2008- \\
2009\end{array}$ & $\begin{array}{l}2008- \\
2009\end{array}$ & $\begin{array}{l}2007- \\
2009\end{array}$ & $\begin{array}{l}2007- \\
2009\end{array}$ & $\begin{array}{l}2007- \\
2009\end{array}$ & $\begin{array}{l}2007- \\
2009\end{array}$ & $\begin{array}{l}2007- \\
2009\end{array}$ & $\begin{array}{l}2007- \\
2009\end{array}$ & $\begin{array}{l}2007- \\
2009\end{array}$ & $\begin{array}{l}2007- \\
2009\end{array}$ & $\begin{array}{l}2007- \\
2009\end{array}$ & 2009 & 2008 \\
\hline & Unit & $\mathrm{pg} / \mathrm{m} 3$ & $\mathrm{pg} / \mathrm{m} 3$ & $\mathrm{ng} / \mathrm{g}$ & $\mathrm{ng} / \mathrm{g}$ & $\mathrm{ng} / \mathrm{g}$ & $\mathrm{ng} / \mathrm{g}$ & $\mathrm{ng} / \mathrm{g}$ & $\mathrm{ng} / \mathrm{g}$ & $\mathrm{ng} / \mathrm{g}$ & $\mathrm{ng} / \mathrm{g}$ & $\mathrm{ng} / \mathrm{g}$ & $\mathrm{pg} / \mathrm{m} 3$ & $\mathrm{ng} / \mathrm{m} 3$ \\
\hline \multirow[t]{14}{*}{ Telomers } & 4:2 FTOH & & & & & & & & & & & & & \\
\hline & 6:2-FTOH & & & & & & & & & & & & & 0.59 \\
\hline & 8:2-FTOH & & & & & & & & & & & & 4,839 & 10.16 \\
\hline & 10:2-FTOH & & & & & & & & & & & & 2,610 & 2.29 \\
\hline & 8:2-FTOHAC & & & & & & & & & & & & & 0.34 \\
\hline & 8:2-FтОНМас & & & & & & & & & & & & & 0.05 \\
\hline & EtFOSA & 120 & 59 & 98 & 120 & 2,000 & 1,300 & 150 & 190 & 150 & 140 & 140 & 5.3 & \\
\hline & MeFOSA & $<2.5$ & 6 & 13 & 61 & 360 & 32 & 5.4 & 1.7 & $<0.1$ & 1.6 & 15 & & \\
\hline & EtFOSE & 600 & 490 & 320 & 290 & 60 & 8.4 & 190 & 100 & 5.7 & 59 & 210 & 27 & \\
\hline & MeFOSE & 950 & 480 & 230 & 250 & 84 & 8.4 & 190 & 84 & 12 & 14 & 120 & 8.3 & \\
\hline & MeFOSEA & & & & & & & & & & & & 6.9 & \\
\hline & FOSA & 152 & 74 & 54 & 21 & 25 & 190 & 3.4 & 56 & $<0.02$ & 13 & 66 & & \\
\hline & PFBS & & & & & & & & & & & & & \\
\hline & PFHXS & 36 & 94 & 450 & 620 & 240 & 150 & 130 & 290 & 94 & 25 & 270 & & \\
\hline
\end{tabular}





\section{Appendix F - Data contributions of PFCA and PFSA in food and drinking water}


Table 1. PFCA in food and drinking water

Concentration, mean (range) of perfluorocarboylates, PFCA, in food (ng/kg or ug/ $\mathrm{kg}$ ) and drinking water (ng/L)

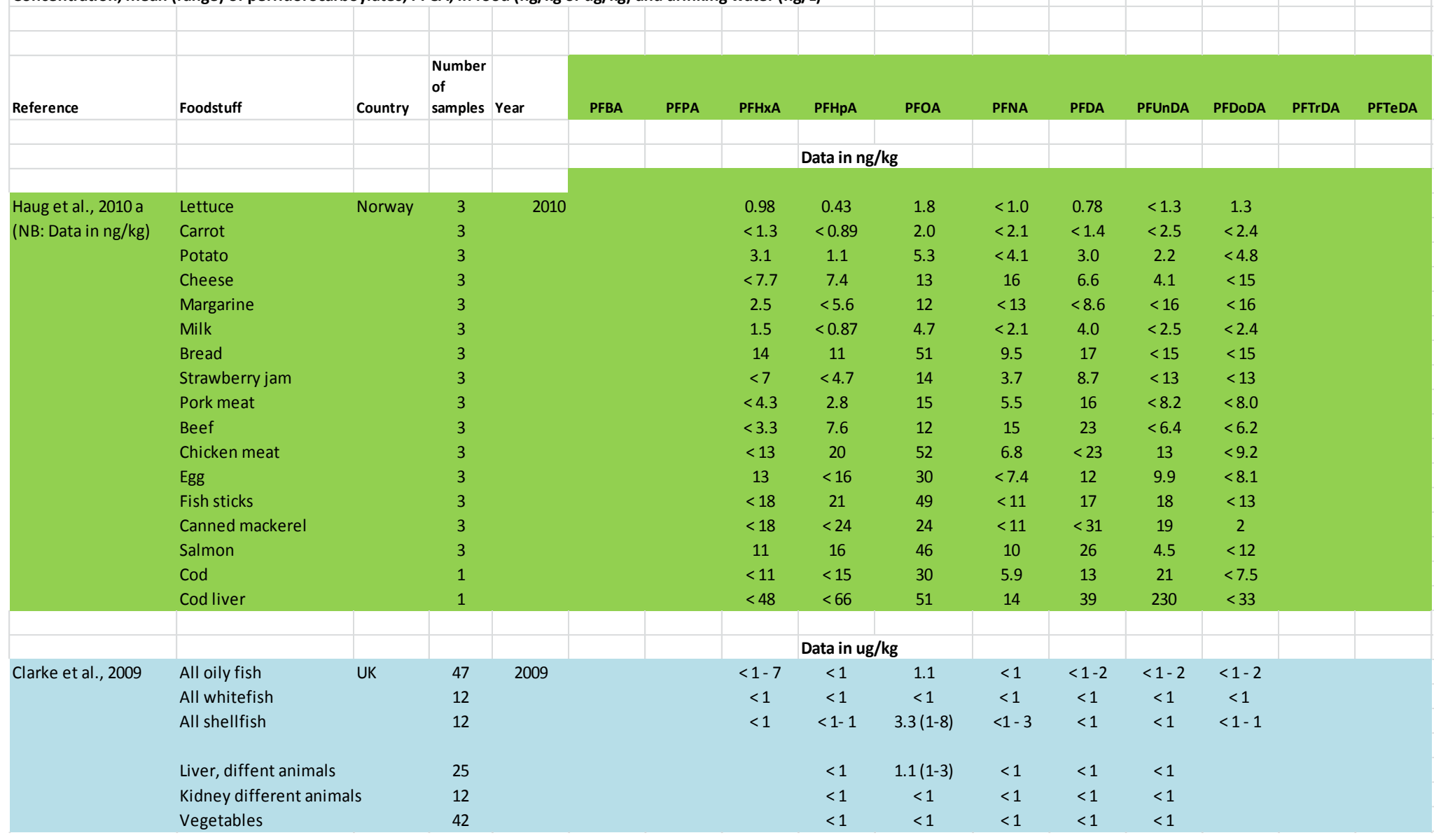


Table 2. PFCA in food and drinking water

Concentration, mean (range) of perfluorocarboylates, PFCA, in food (ng/kg or ug/kg) and drinking water (ng/L)

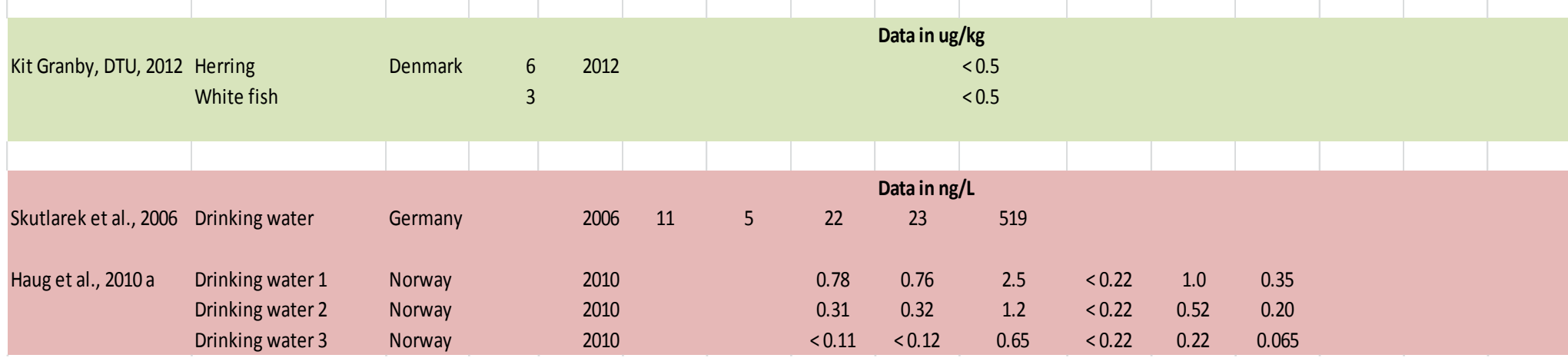


Table 3. PFSA in food and drinking water

Concentration, mean (range), of perfluoroalkyl sulfonates, PFSA, and perfluoroalkyl sulfonic Acid Amides in food (ng/kg or ug/kg) and drinking water (ng/L)

\begin{tabular}{|c|c|c|c|c|c|c|c|c|c|c|}
\hline Reference & Foodstuff & Country & $\begin{array}{l}\text { Number } \\
\text { of samples Year }\end{array}$ & PFBS & PFHXS & PFHpS & PFOS & PFDS & PFOSA & $\sum$ PFC's \\
\hline & & & & & ata in $\mathrm{ng} / \mathrm{kg}$ & & & & & \\
\hline \multirow[t]{19}{*}{ Haug et al., 2010} & Lettuce & Norway & 3 & $<0.12$ & $<0.06$ & & 0.17 & & & \\
\hline & Carrot & & 3 & $<0.25$ & $<0.1$ & & 0.67 & & & \\
\hline & Potato & & 3 & $<0.48$ & $<0.22$ & & 1.0 & & & \\
\hline & Cheese & & 3 & $<1.5$ & $<0.6$ & & 12 & & & \\
\hline & Margarine & & 3 & $<1.6$ & 1.3 & & 2. & & & \\
\hline & Milk & & 3 & $<0.24$ & $<0.1$ & & 7.0 & & & \\
\hline & Bread & & 3 & $<1.5$ & 1.7 & & 17 & & & \\
\hline & Strawberry jam & & 3 & $<1.3$ & $<0.59$ & & 3.0 & & & \\
\hline & Pork meat & & 3 & $<0.81$ & 1.2 & & 17 & & & \\
\hline & Beef & & 3 & $<0.63$ & $<0.28$ & & 60 & & & \\
\hline & Chicken meat & & 3 & 3.2 & 2.3 & & 21 & & & \\
\hline & Egg & & 3 & 2.0 & 3.5 & & 39 & & & \\
\hline & Fish sticks & & 3 & 5.0 & 1.6 & & 13 & & & \\
\hline & Canned mackerel & & 3 & 5.5 & $<3$ & & 43 & & & \\
\hline & Salmon & & 3 & 2.2 & 5.5 & & 55 & & & \\
\hline & Cod & & 1 & $<3.4$ & 2.8 & & 100 & & & \\
\hline & Cod liver & & 1 & $<15$ & $<8.2$ & & 310 & & & \\
\hline & & & & & & & & & & \\
\hline & & & & & ata in ug/kg & & & & & \\
\hline \multirow[t]{6}{*}{ Clarke et al., 2009} & All oily fish & Germany & 47 & $<1$ & $<1-1$ & & $4.8(<1-59)$ & & $2.5(1-27)$ & $6.7(0-63)^{*}$ \\
\hline & All whitefish & & 12 & $<1$ & $<1$ & & $1.2(<1-2)$ & & $1.1(1-2)$ & $0.8(0-4)^{*}$ \\
\hline & All shellfish & & 12 & & $<1-2$ & & $4.4(1-13)$ & & $1.3(1-3)$ & $8.2(0-20)^{*}$ \\
\hline & Liver, diffent animals & & & $<1$ & $<1$ & & $2.5(1-10)$ & & $<1$ & $2.4(0-14)^{*}$ \\
\hline & Kidney different animals & & & $<1$ & $<1$ & & $1.4(1-3)$ & & $<1$ & $12(0-5)^{*}$ \\
\hline & Vegetables & & & $<1-1$ & $<1$ & & $<1$ & & $<1$ & $0.1(0-2)^{*}$ \\
\hline
\end{tabular}

Note $\sum$ PFC's: ${ }^{*}=\sum$ PFHxA, PFHpA, PFOA, PFNA, PFDeA, PFUnA, PFDoA, PFBS, PFHxS, PFOS and PFOSA 
Concentration, mean (range), of perfluoroalkyl sulfonates, PFSA, and perfluoroalkyl sulfonic Acid Amides in food (ng/kg or ug/kg) and drinking water (ng/L)

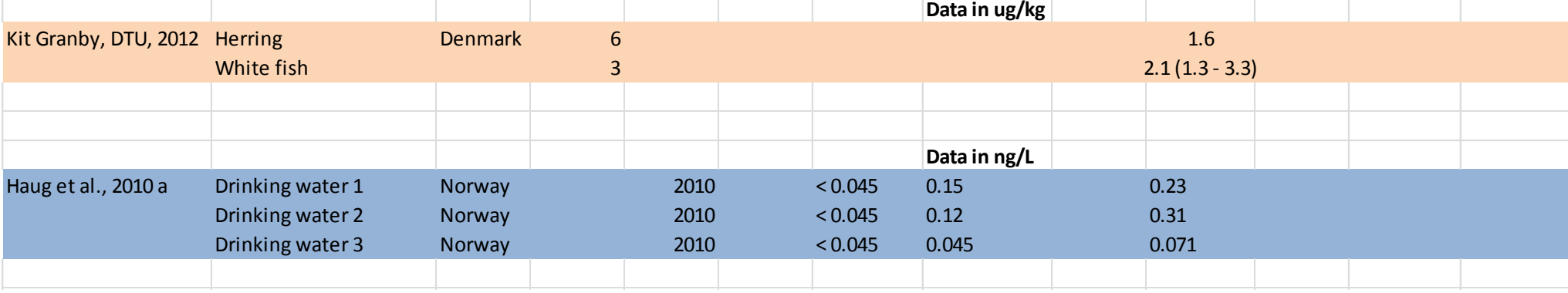




\section{norden}

Nordic Council of Ministers

Ved Stranden 18

DK-1061 Copenhagen K

www.norden.org

\section{Per- and polyfluorinated substances in the Nordic Countries}

This Tema Nord report presents a study based on open information and custom market research to review the most common perfluorinated substances (PFC) with less focus on PFOS and PFOA.

The study includes three major parts:

1. Identification of relevant per-and polyfluorinated substances and their use in various industrial sectors in the Nordic market by interviews with major players and database information

2. Emissions to and occurence in the Nordic environment of the substances described in 1)

3. A summary of knowledge of the toxic effects on humans and the environment of substances prioritized in 2)

There is a lack of physical chemical data, analystical reference substances, human and environmental occurrence and toxicology data, as well as market information regarding PFCs other than PFOA and PFOS and the current legislation cannot enforce disclosure of specific PFC substance information.

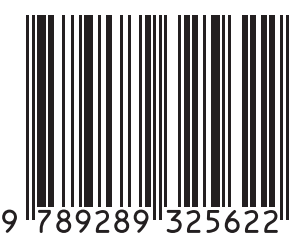

